\title{
MRI-based tailored treatment of rectal cancer : the impact on surgical outcome
}

Citation for published version (APA):

Engelen, S. M. E. (2009). MRI-based tailored treatment of rectal cancer : the impact on surgical outcome. [Doctoral Thesis, Maastricht University]. Maastricht University. https://doi.org/10.26481/dis.20090327se

Document status and date:

Published: 01/01/2009

DOI:

10.26481/dis.20090327se

Document Version:

Publisher's PDF, also known as Version of record

\section{Please check the document version of this publication:}

- A submitted manuscript is the version of the article upon submission and before peer-review. There can be important differences between the submitted version and the official published version of record.

People interested in the research are advised to contact the author for the final version of the publication, or visit the DOI to the publisher's website.

- The final author version and the galley proof are versions of the publication after peer review.

- The final published version features the final layout of the paper including the volume, issue and page numbers.

Link to publication

\footnotetext{
General rights rights.

- You may freely distribute the URL identifying the publication in the public portal. please follow below link for the End User Agreement:

www.umlib.nl/taverne-license

Take down policy

If you believe that this document breaches copyright please contact us at:

repository@maastrichtuniversity.nl

providing details and we will investigate your claim.
}

Copyright and moral rights for the publications made accessible in the public portal are retained by the authors and/or other copyright owners and it is a condition of accessing publications that users recognise and abide by the legal requirements associated with these

- Users may download and print one copy of any publication from the public portal for the purpose of private study or research.

- You may not further distribute the material or use it for any profit-making activity or commercial gain

If the publication is distributed under the terms of Article $25 \mathrm{fa}$ of the Dutch Copyright Act, indicated by the "Taverne" license above, 


\section{MRI-BASED TAILORED TREATMENT OF RECTAL CANCER}

the impact on surgical outcome 


\section{MRI-BASED TAILORED TREATMENT OF RECTAL CANCER}

the impact on surgical outcome

\section{PROEFSCHRIFT}

ter verkriging van de graad van doctor

aan de Unversiteit Maastricht,

op gezag van de Rector Magnificus

Prof. mr. G.P.M.F. Mols

volgens het besluit van het College van Decanen

in het openbaar te verdedigen

op vrijdag 27 maart 2009 om 14.00 uur

door

SANNE MARIE ELISE ENGELEN

geboren op 14 juli 1980 te Boxmeer 


\title{
Promotores:
}

Prof. dr. R.G.H. Beets-Tan

Prof. dr. C.J.H. van de Velde (LUMC Leiden)

Prof. dr. M.F. von Meyenfeldt

Co-promotor:

Dr. G.L. Beets

Beoordelingscommissie:

Prof. dr. C.H.C. Dejong (voorzitter)

Prof. dr. R.F.P.M. Kruitwagen

Prof. dr. S. Stroobants (UZ Antwerpen)

Prof. dr. J.E. Wildberger

Dr. J.H.W. de Wilt (Erasmus MC Rotterdam)

\author{
Additional financial support was \\ provided by: \\ Bayer Schering Pharma, Guerbet, \\ Roche Nederland B.V.
}

(C) S.M.E. Engelen, Maastricht 2009

All rights reserved. No part of this book may be translated or reproduced in any

form by photo, photo print, microfilm or any other means without written

permission from the author.

Financial support by the Dutch Cancer Society for this study and the publication of this thesis is gratefully acknowledged.

ISBN: $978-90-713-8297-0$

Lay-out omslag: Gildeprint, Enschede

Druk: Gildeprint, Enschede 


\section{CONTENTS}

$\begin{array}{lll}\text { Chapter } 1 \text { General introduction } & 7\end{array}$

Chapter 2 Role of preoperative local and distant staging in rectal cancer: a review

Chapter 3 Location of involved mesorectal and extramesorectal lymph nodes in patients with primary rectal cancer: preoperative assessment with MR imaging

Chapter $4 \quad$ USPIO MRI for nodal staging of patients with primary rectal cancer: predictive criteria

Chapter 5 MR prediction of the risk factors - CRM, T-stage and nodal status - in primary rectal cancer: a multicenter study

Chapter 6 MRI-based tailored treatment of rectal cancer: impact on surgical outcome

Chapter 7 Aggressive multidisciplinary treatment of locally advanced or unresectable rectal cancer. Local control is good, but patients still die of metastases

Chapter 8 MRI after chemoradiotherapy of rectal cancer:

a useful tool to select patients for local excision

Chapter 9 Summary, discussion and conclusions

Chapter $10 \quad$ Samenvatting

Dankbetuigingen

Curriculum Vitae 
Ceneral Introduction 


\section{Chapter 1}

Colorectal cancer was diagnosed in almost 300,000 patients in Europe in 2006. It is the third most common cancer in men and women together, and the second most common cause of cancer-related death ${ }^{1,2}$. About $30 \%$ of all colorectal cancers is located in the rectum. Apart from cancer-related death, rectal cancer is especially notorious for local recurrence, which often comes with severe morbidity. The treatment of rectal cancer therefore focuses on optimal local and distant control. In the past decades, this treatment has undergone major developments both in surgery and neoadjuvant therapy. With regard to surgery, the introduction of Total Mesorectal Excision (TME) has significantly reduced the local recurrence rate ${ }^{3}$. In this TME the rectum is removed together with its surrounding fat including lymph nodes and blood vessels through which tumor cells can spread (which is called the mesorectum), with a sharp surgical technique along the outside of the mesorectal fascia, a fascia that envelops this mesorectum. This improvement in surgical technique was also driven by the notion that the distance of the tumor to the circumferential resection margin (CRM), as judged by the pathologist, was a very important factor in local recurrence rates ${ }^{4}$. At the same time Scandinavian trials have shown that preoperative radiotherapy additional to conventional surgery is more effective than postoperative radiotherapy for reduction of local recurrence ${ }^{5,6}$. The Dutch TME trial ${ }^{7}$ as well as the MRC CRO7 trial $^{8}$ furthermore showed that even with good TME surgery there is a favorable effect of neoadjuvant $5 \times 5$ Gy radiation therapy on local recurrence rates for the whole group, so in the Netherlands and in Scandinavia $5 \times 5$ Gy before TME has become the standard treatment for all rectal cancer patients. A subgroup analysis of the Dutch TME trial also showed that a short course of preoperative radiation ( $5 \times 5 \mathrm{~Gy}$ ) does not improve outcome in stage I tumors ( $T_{1-2} \mathrm{No}$ ) on one hand, as these tumors have already a favourable prognosis without radiotherapy ${ }^{7}$. Because it was a subgroup analysis, and because of the difficulties with nodal staging, clinicians were reluctant to withhold radiotherapy in these patients. For the more advanced tumors on the other hand, which are tumors with an involved circumferential resection margin $(C R M)$, it was shown that the recurrence rates were too high, even after a preoperative short course of radiation ${ }^{9}$. To avoid local recurrences in this group, more aggressive neoadjuvant treatment has been advocated. In 1991 Krook et 
al. ${ }^{10}$ showed the advantage of addition of chemotherapy to adjuvant radiotherapy for reduction of local recurrence and improvement of overall survival for more advanced tumors. Two other studies proposed chemotherapy plus radiation therapy in a neoadjuvant setting, as it not only improved the local control $^{11,}{ }^{12}$, but also showed a better adherence to treatment as compared to postoperative chemotherapy ${ }^{12}$. In 2004, Saver et al. ${ }^{13}$ confirmed that this combined chemoradiation is more effective in terms of local control and toxicity when given preoperatively as compared to postoperatively. Locally advanced rectal cancer is therefore preferably treated with a long course neoadjuvant radiation with chemotherapy as a radiosensitizer.

The selection of these 'more advanced tumors' with a high risk for circumferential margin involvement has long been made based on clinical examination ("fixed tumors") and/or endosonography when available. Clinical and endosonographic staging is not very accurate for assessing tumors with involved circumferential resection margin. In the Dutch TME trial, where the intent was to exclude clinically fixed ('locally advanced') tumors, there was a rate of involved margins of $16 \%$, often due to the inclusion of very large 'advanced tumors' that were understaged. With the introduction of MRI since the beginning of this millennium in the work-up of rectal cancer patients the mesorectal fascia -the anticipated circumferential resection margin- could accurately be visualised. Tumors that were growing into or very near to the mesorectal fascia could be better identified ${ }^{14-19}$, as shown in single center studies in expert center, but also confirmed in a multicenter study in general setting ${ }^{20}$. This has been an important development because of the importance of this circumferential resection plane had been established previously ${ }^{4}$.

Apart from the circumferential resection margin, the presence of nodal disease is an important risk factor for local recurrence. Until recently, imaging modalities lacked the accuracy for nodal prediction, because the main prediction criterion used was size, and in rectal cancer lymph node metastases have been known to occur in nodes smaller than $5 \mathrm{~mm}$ as well ${ }^{21}$. The introduction of lymph nodespecific contrast agents for MRI such as USPIO (Ultrasmall Paramagnetic Iron Oxide) offered the hope of better lymph node prediction, and in prostate as well as head and neck cancer a sensitivity and specificity of up to 0.88 and 0.96 respectively was reported ${ }^{22}$. In a pilot study in our center with a lesion by lesion 


\section{Chapter 1}

analysis with histology as validation we confirmed these promising results also in rectal cancer patients ${ }^{23}$. These developments in MR imaging in accurate prediction of circumferential resection margin as well as promising results with regard to nodal staging could lead to a more differentiated treatment approach in rectal cancer rather than one uniform treatment for all. From all available treatment options the one can be chosen that best fits an individual patient, based on an assessment of the risk for local recurrence. Theoretically this differentiated treatment leads to an optimal local control with minimal treatment related morbidity for the whole group of rectal cancer patients.

Good classification systems that group together patients with similar prognoses are essential. It provides a uniform 'language' to compare outcomes in different studies, to pool together similar patients to increase the statistical power to detect meaningful improvements, and to provide guidelines on how best to treat patients. The universally accepted staging system is the TNM system ${ }^{24}$. It is however unfortunate that for rectal cancer some of the important prognostic factors for local recurrence are not well captured in the TNM system for rectal cancer (see table 1.1). The distance of the tumor to the mesorectal fascia and the distance of the tumor to the anal verge are not part of the staging system. The TNM staging system basically groups together patients with similar overall survival, dictated mostly by distant metastases, and is less focused on local recurrence.

\begin{tabular}{ll}
\hline AJCC Stage & TNM \\
\hline I & T1-2NoMo \\
II & $T_{3-4 N o M o}$ \\
III & $\mathrm{T}_{1-4 N 1-2 \mathrm{Mo}}$ \\
IV & $\mathrm{T}_{1-4} \mathrm{No}_{2} \mathrm{M}_{1}$ \\
\hline Table 1.1 AJCC stage and TNM staging system
\end{tabular}

A differentiated treatment approach that addresses optimal local treatment will have to use a classification system that includes more prognostic variables than the TNM system. The scientific evidence for these other variables is more recent, and less solid. This is reflected in the large variation in protocols and guidelines, and many unsuccessful attempts to come to a uniform assessment and treatment system. A good example is the term 'locally advanced'. In some 
countries $\mathrm{T}_{3}$ or $\mathrm{N}_{1}$ disease is called locally advanced, whereas in others this term is reserved for tumors that are growing into surrounding organs ${ }^{25}$.

At the basis of many papers in this thesis is a large regional project on rectal cancer, funded by the Dutch Cancer Society, in which four hospitals participated. The three basic treatment options that were available at the start of the project were $5 \times 5$ Gy RT and TME surgery, which was the recommendation for the majority of patients after the Dutch TME trial, TME surgery without neoadjuvant therapy, and a long course of chemoradiation followed by surgery. The basic idea was that with a good preoperative MRI the local recurrence results of the Dutch TME trial could be further improved, mainly by identifying tumors that were at risk for a positive CRM, and treating these with neoadjuvant chemoradiation rather than $5 \times 5 \mathrm{~Gy}$. Additionally there was the feeling that some patients with 'low risk' tumors were overtreated with radiotherapy. In consensus with the four participating hospitals and using the available knowledge at the time, it was discussed and decided how best to classify patients into three different risk groups, with three different treatment policies matching the risk (see figure 1.1).

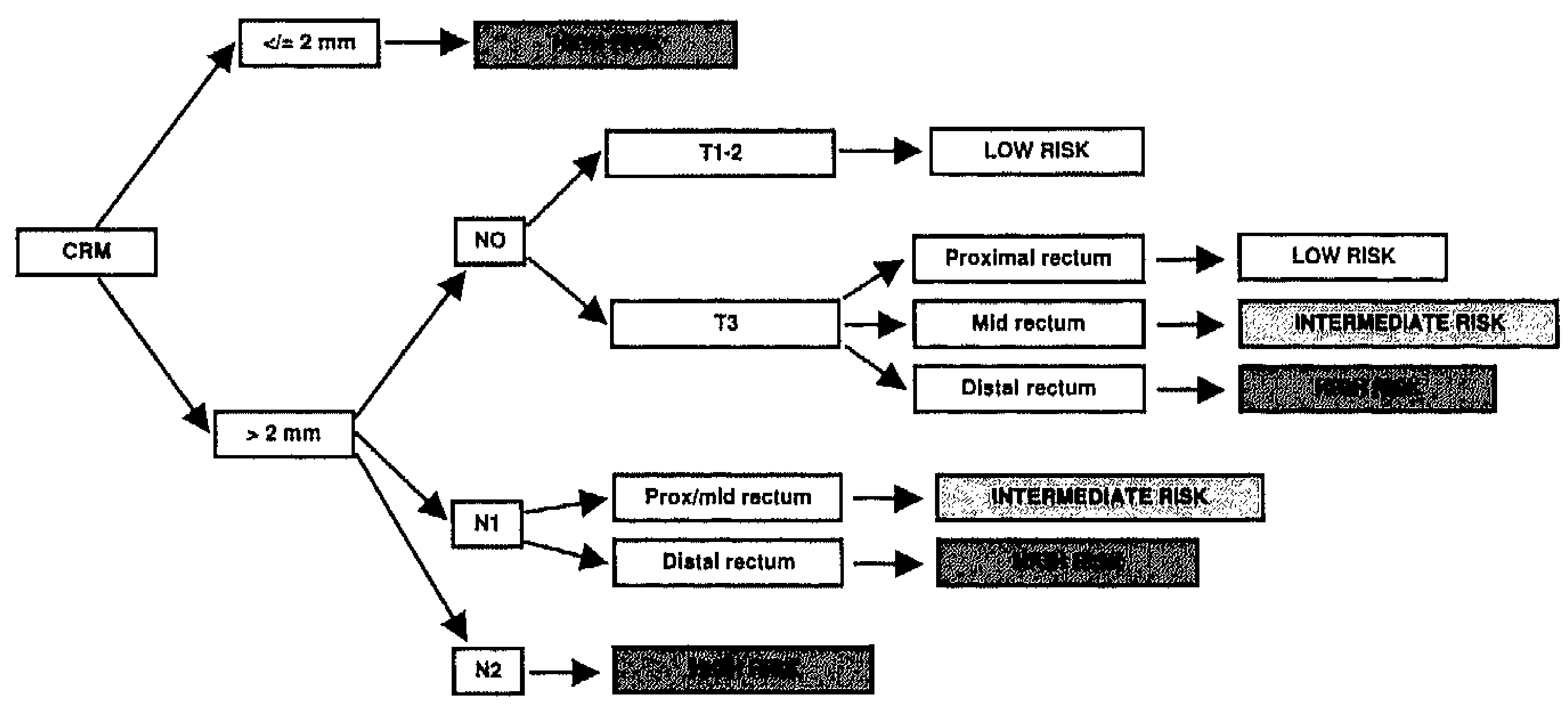

Figure 1.1 MRI-based decision scheme for tailored treatment of rectal cancer

The most weight was given to the well established risk factor of an anticipated CRM on MRI. It was acknowledged that the accuracy of MRI in lymph node staging was less well established, and this prognostic factor was given less 
weight. The general feeling was that in case of doubt, overtreatment was less harmful than undertreatment. In addition to the main aim of the project to improve the outcome with regard to local recurrence rates by selecting and treating the 'high risk' patients for neoadjuvant chemoradiation, the project provided an opportunity to study a new MR contrast agent for lymph node imaging and other related issues in various subsets of patients. As the patients in chapters 4,6 and 8 , addressing these questions are all part of the large regional project, there is inevitably some overlap between patients groups and methodology.

\section{THE PRIMARY AIM OF THIS THESIS IS:}

To evaluate whether the use of preoperative MRI as a selection tool for the different risk groups of rectal cancer patients and the subsequent differentiated treatment leads to an improved surgical outcome in a prospective multicenter cohort of rectal cancer patients.

\section{THE SECONDARY AIMS OF THIS THESIS ARE:}

- To evaluate the accuracy of MRI with a lymph node-specific contrast agent for the prediction of tumor stage and nodal disease.

* To evaluate the results of a multimodal aggressive treatment for locally advanced disease as defined by an expected involved CRM on preoperative imaging.

- To define whether imaging could lead to an even more differentiated treatment in good responders. 
General Introduction

\section{THE OUTLINE OF THIS THESIS:}

Chapter 2 reviews the current status of preoperative local and distant staging in primary rectal cancer.

Chapter 3 focuses on the distribution of malignant mesorectal and extramesorectal lymph nodes in patients with rectal cancer.

Chapter 4 evaluates the criteria for the prediction of metastatic involvement of lymph nodes with USPIO-enhanced MRI. It is a prospective single center study that uses a lesion-by-lesion analysis with histology as the reference standard. Patients who are treated with chemoradiation are not included because the histology after resection does no longer represent the initial status.

Chapter 5 focuses on the accuracy of MRI on a patient basis in predicting CRM, T\& $\mathrm{N}$-stage at the primary presentation in a prospective multicenter cohort study. Patients who are treated with chemoradiation are excluded because the histology after resection does no longer represent the initial status.

Chapter 6 reports on the main aim of the prospective multicenter regional MR project: the impact on surgical outcome of a differentiated treatment of rectal cancer patients based on preoperative selection of low, medium and high risk groups with MR imaging.

Chapter 7 retrospectively describes the results of an aggressive multimodality approach for treatment of locally advanced rectal cancer in a referral center with a view on future perspectives regarding the treatment of these patients.

Chapter 8 discusses the accuracy of MRI in restaging after chemoradiation to select ypTo-2No patients, and explores the possible future application of this in selecting patients for a less invasive treatment such as a local excision.

In chapter 9, all results will be discussed and recommendations for clinical patient care and future research will be given. 


\section{Chapter 1}

\section{REFERENCES}

1.

Ferlay J, Autier $\mathrm{P}$, Boniol $\mathrm{M}$, Heanue $\mathrm{M}$, Colombet $\mathrm{M}$, Boyle $\mathrm{P}$. Estimates of the cancer incidence and mortality in Europe in 2006. Ann Oncol 2007;18(3): $581-592$.

2. Boyle P, Ferlay J. Mortality and survival in breast and colorectal cancer. Nat Clin Pract Oncol $2005 ; 2(9): 424-425$.

3. Heald RJ, Ryall RD. Recurrence and survival after total mesorectal excision for rectal cancer. Lancet 1986;1(8496): 1479-1482.

4. Nagtegaal ID, Quirke P. What is the role for the circumferential margin in the modern treatment of rectal cancer? J Clin Oncol 2008;26(2): 303-312.

5. Frykholm GJ, Glimelius B, Pahlman L. Preoperative or postoperative irradiation in adenocarcinoma of the rectum: final treatment results of a randomized trial and an evaluation of late secondary effects. Dis Colon Rectum 1993;36(6): 564-572.

6. Improved survival with preoperative radiotherapy in resectable rectal cancer. Swedish Rectal Cancer Trial. N Engl J Med 1997;336(14): 980-987.

7. Kapiteijn E, Marijnen CA, Nagtegaal ID, Putter H, Steup WH, Wiggers T, Rutten HJ, Pahlman L, Glimelius $B$, van Krieken $J H$, Leer JW, van de Velde CJ. Preoperative radiotherapy combined with total mesorectal excision for resectable rectal cancer. N Engl J Med 2001;345(9): 638-646.

8. Sebag-Montefiore D, Steele RJ, Quirke P, Grieve R, Khanna S, Monson JR, Holliday A, Thompson $L_{\iota}$ Griffiths G, Stephens R. Routine short course pre-op radiotherapy or selective post-op chemoradiotherapy for resectable rectal cancer? Preliminary results of the MRC CR 07 randomized trial. Journal of Clinical Oncology 2006;24(18S): 3511.

9. Marijnen CA, Nagtegaal ID, Kapiteijn E, Kranenbarg EK, Noordijk EM, van Krieken JH, van de Velde $\mathrm{CJ}$, Leer JW. Radiotherapy does not compensate for positive resection margins in rectal cancer patients: report of a multicenter randomized trial. Int J Radiat Oncol Biol Phys 2003;55(5): 1311-1320. Krook JE, Moertel CG, Gunderson LL, Wieand HS, Collins RT, Beart RW, Kubista TP, Poon MA, Meyers WC, Mailliard JA, et al. Effective surgical adjuvant therapy for high-risk rectal carcinoma. $N$ Engl J Med 1991;324(11): 709-715.

11. Bosset JF, Collette L, Calais G, Mineur L, Maingon P, Radosevic-Jelic L, Daban A, Bardet E, Beny A, Ollier JC. Chemotherapy with preoperative radiotherapy in rectal cancer. N Engl J Med 2006;355(11): $1114-1123$.

12. Gerard JP, Conroy T, Bonnetain $F$, Bouche $O$, Chapet $O$, Closon-Dejardin MT, Untereiner M, Leduc B, Francois E, Maurel J, Seitz JF, Buecher B, Mackiewicz R, Ducreux M, Bedenne L. Preoperative radiotherapy with or without concurrent fluorouracil and leucovorin in T3-4 rectal cancers: results of FFCD 9203. J Clin Oncol 2006;24(28): 4620-4625.

13. Saver R, Becker $H_{1}$, Hohenberger W, Rodel C, Wittekind $C$, Fietkau R, Martus $P$, Tschmelitsch J, Hager E, Hess CF, Karstens JH, Liersch T, Schmidberger H, Raab R. Preoperative versus postoperative chemoradiotherapy for rectal cancer. N Engl J Med 2004;351(17): 1731-1740.

14. Beets-Tan RG, Beets GL, Vliegen RF, Kessels AG, Van Boven $H$, De Bruine A, von Meyenfeldt MF Baeten $C G$, van Engelshoven $J M$. Accuracy of magnetic resonance imaging in prediction of tumourfree resection margin in rectal cancer surgery. Lancet 2001;357(9255): 497-504.

15. Brown G, Richards CJ, Newcombe RG, Dallimore NS, Radcliffe AG, Carey DP, Bourne MW, Williams GT. Rectal Carcinoma: Thin-Section MR Imaging for Staging in 28 Patients. Radiology 1999;211(1): 215-222.

16. Beets-Tan RG, Beets GL. Rectal cancer: how accurate can imaging predict the $T$ stage and the circumferential resection margin? Int J Colorectal Dis 2003;18(5): 385-391.

17. Bissett IP, Fernando CC, Hough DM, Cowan BR, Chau KY, Young AA, Parry BR, Hill GL. Identification of the fascia propria by magnetic resonance imaging and its relevance to preoperative assessment of rectal cancer. Dis Colon Rectum 2001;44(2): 259-265.

18. Blomqvist L, Machado M, Rubio C, Gabrielsson N, Granqvist S, Goldman S, Holm T. Rectal tumour staging: MR imaging using pelvic phased-array and endorectal coils vs endoscopic ultrasonography. Eur Radiol $2000 ; 10(4): 653-660$. 
19. Peschaud F, Cuenod CA, Benoist S, Julie C, Beauchet A, Siauve N, Taieb-Kasbi F, Penna C, Nordlinger $B$. Accuracy of magnetic resonance imaging in rectal cancer depends on location of the tumor. Dis Colon Rectum 2005;48(8): 1603-1609.

20. Salerno G, Daniels IR, Moran BJ, Wotherspoon A, Brown G. Clarifying margins in the multidisciplinary management of rectal cancer: the MERCURY experience. Clin Radiol 2006;61(11): 916-923.

21. Wang $C$, Zhou Z, Wang $Z$, Zheng $Y$, Zhao G, Yu Y, Cheng Z, Chen D, Liu W. Patterns of neoplastic foci and lymph node micrometastasis within the mesorectum. Langenbecks Arch Surg 2005;390(4): 312318.

22. Will O, Purkayastha S, Chan C, Athanasiou T, Darzi AW, Gedroyc W, Tekkis PP. Diagnostic precision of nanoparticle-enhanced MRI for lymph-node metastases: a meta-analysis. Lancet Oncol 2006;7(1): $52-60$.

23. Lahaye MJ, Engelen SM, Beets GL, de Bruine AP, von Meyenfeldt MF, Van Engelshoven JM, Van de Velde CJ, Beets-Tan RG. How accurate can we predict the nodal status in primary rectal cancer with USPIO MRI? Ann Oncol 2006;17 (suppl); i11-12.

24. Greene F, Balch CP, D. AJCC Cancer Staging Manual (6th edn). Springer Verlag: New York, 2002.

25. Glimelius $B$, Holm T, Blomqvist L. Chemotherapy in addition to preoperative radiotherapy in locally advanced rectal cancer - a systematic overview. Rev Recent Clin Trials 2008;3(3): 204-211. 


\section{Role of preoperative local and distant staging in rectal cancer: a review}

S.M.E. Engelen, G.L. Beets, R.G.H. Beets-Tan

(Onkologie. 2007; 30:141-145.) 


\section{Chapter 2}

\section{ABSTRACT}

Preoperative imaging in rectal cancer is very important, as accurate staging determines optimal treatment strategy. In this review, imaging modalities for locoregional and distant staging in rectal cancer are discussed.

For local staging, superficial tumors are best staged using EUS, as EUS is the most accurate modality for assessment of tumor ingrowth into the rectal wall layers. The more advanced tumors are best imaged using MRI, because MRI accurately predicts the distance from tumor to mesorectal fascia, and thus the CRM, as well as possible invasion into surrounding organs.

For the prediction of the nodal status none of the three imaging modalities -EUS, MRI and CT- can be reliably used for clinical decision making. Only MRI using lymph node specific contrast (such as Ultra Small Paramagnetic Iron Oxideenhanced MRI) seems promising for the detection of nodal disease.

For the detection of distant metastases transabdominal ultrasound and chest Xray are used as a primary screening tool. However for the high prevalence group (stage III) both methods are insufficiently sensitive and CT of the chest plus abdomen is preferred. 


\section{INTRODUCTION}

After treatment, rectal cancer can recur locally or systemically, both of which are a threat to the patients' survival. Local recurrence rates were reported to be as high as $32 \%$, but in the last two decades it has become clear that with a combination of standardized surgery according to TME principle ${ }^{2}$ and (neo)adjuvant (chemo-)radiotherapy this can be reduced to $6 \%{ }^{3}$.

Local tumor extent and nodal status are the two most important factors that influence local recurrence rate and can be used to determine optimal treatment ${ }^{4}$. Although classically the local extent has been expressed in $\mathrm{T}$-stage ${ }^{5}$, the circumferential resection margin (CRM) is nowadays considered as at least as important. The circumferential resection margin is the smallest distance from tumor to mesorectal fascia (the plane of resection) when viewed in transverse section through the rectum. The circumferential resection margin is very well correlated with chance for local recurrence: the smaller the margin the higher the risk $^{6}$.

Regarding nodal metastases it is important to realize that in the majority of rectal cancers lymphatic invasion occurs in the mesorectum immediately surrounding the tumor or in the cranial direction along the superior rectal vessels. Distal rectal cancer can also metastasize to lateral nodes, usually in the presence of involved mesorectal nodes ${ }^{7}$. There are several options for the local treatment of rectal cancer. TME surgery has become the standard, but for some very superficial tumors transanal endoscopic microsurgery (TEM) is sometimes advocated as a less invasive alternative. Each of these procedures can be preceded by either a short course of radiotherapy or chemoradiation. To make a rational choice good local imaging can be of help to distinguish between early low risk tumors (wide circumferential resection margin, No status) and the more advanced high risk tumors (close or involved circumferential resection margin, nodal status, extramesorectal disease).

Distant metastases of rectal cancer are mainly located in the liver, and to a lesser extent in the lungs. Synchronous hepatic metastases occur in $12-24 \%$ of patients, with another $8-30 \%$ of patients developing metachronous liver metastases. Pulmonary metastases are more common in rectal than in colon cancer, and ultimately develop in $11-30 \%$ of patients ${ }^{8-10}$. With increasingly effective systemic 


\section{Chapter 2}

and local treatment of metastatic disease a more active search for metastases is often advocated. In the follow up setting after a curative treatment of colorectal cancer a recent meta-analysis shows a mean 5 year survival advantage of $7 \%$ in favour of an active search for metastatic disease ${ }^{10}$. It is likely, but unproven, that identification of metastatic disease as early as possible, ideally at the time of diagnosis of the primary tumor, will lead to improved patient survival due to early treatment of the metastatic disease $\mathrm{s}^{11}$.

In this article, imaging modalities for locoregional and distant staging will be discussed.

\section{LOCOREGIONAL STAGING}

For locoregional staging of rectal carcinoma, Endorectal US and MRI are mainly used. EUS is very accurate in assessing depth of tumor growth in more superficial tumors, confined to the bowel wall with an accuracy for assessment of T-stage between $69 \%$ and $97 \% \%^{4}$. Because of its limited field of view, EUS does not clearly show the mesorectal fascia, making prediction of CRM involvement more difficult. EUS is therefore not very useful for staging the more advanced tumors. Another drawback of EUS is the inability to stage higher and/ or stenosing tumors.

Modern MRI techniques with a dedicated phased-array coil are moderately accurate for prediction of T-stage. On one hand it is very accurate for the prediction of large $T_{3}$ and $T_{4}$ tumors due to its high spatial and contrast resolution, together with a large field of view ${ }^{12}$. On the other hand however it is less accurate for the differentiation between $T_{2}$ and early $T_{3}$ tumors. When $T_{2}$ tumors show spontaneous desmoplastic reaction surrounding the tumor, the MR image may mimic an early $T_{3}$ tumor. On MRI it is hard to distinguish between desmoplastic reaction with and without tumor cells $s^{4}$ (fig 2.1a\&b). Overstaging of pT2 tumors (38-62\%) and understaging of $\mathrm{PT}_{3}$ tumors $(5-18 \%)$ has been reported $^{13}{ }^{14}$. Although at present the differentiation between $T_{2}$ and early $T_{3}$ tumors has little clinical impact, in future when individual based tailored treatment will be performed based on selection by imaging, this differentiation could become important. 
MRI is very accurate for the prediction of the distance from tumor to the mesorectal fascia (fig $2.2 a \& b$ ), because the mesorectal fascia is clearly depicted on MRI. This is shown by the high area under the ROC curve in the meta-analysis

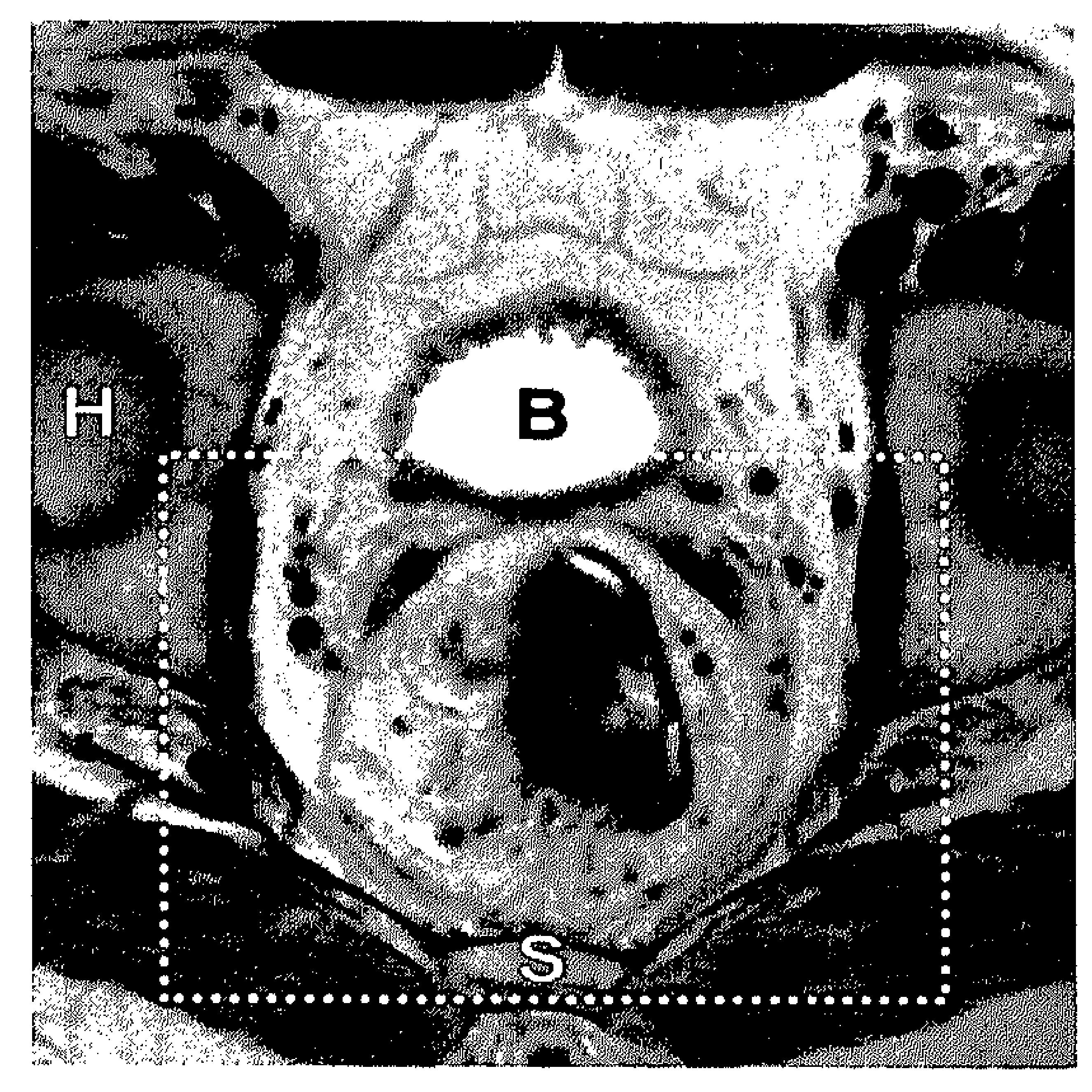

Figure 2.1a Axial T2-weighted fast spin-echo (TR/TE 3427/250 ms) MR image: overview of the pelvis. $B=$ bladder,$H=$ head of femur, $S=$ sacrum

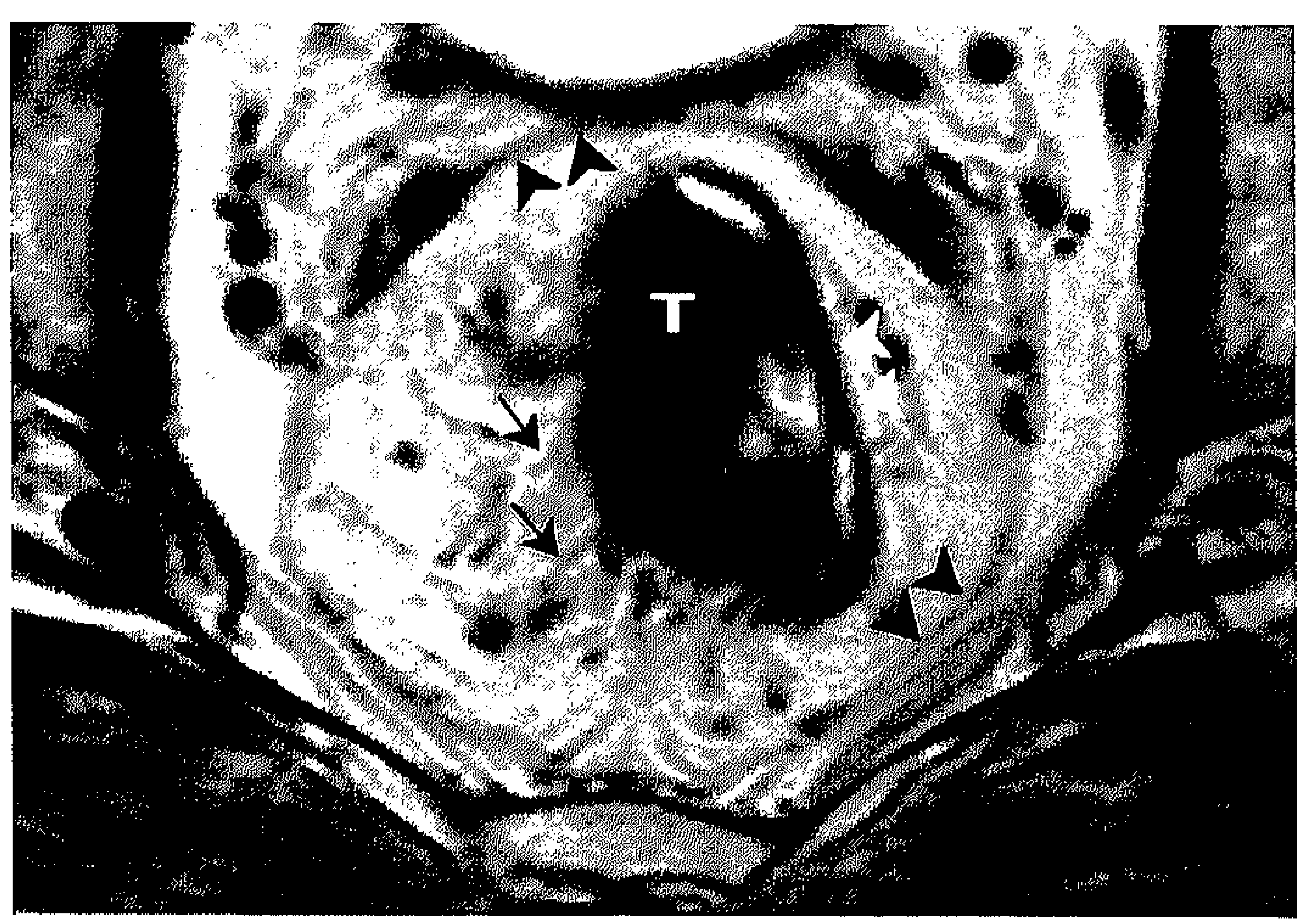

Figure 2.26 Detailed image of selection as indicated in figure 2.1a shows a rectal tumor $(T)$ located from 5 to 12 o'clock (right lateral rectal wall). Tumor shows fibrotic stranding (indicating desmoplastic reaction) into the perirectal fat (black arrows), therefore early $T_{3}$ rectal cancer. On the left lateral side, an intact rectal wall is seen as a black rim (white arrow heads). Distance from tumor to mesorectal fascia (black arrow heads) is wide

of seven single center studies by Lahaye et al ${ }^{15}$. The preliminary results of a large multicenter study, the Mercury Research Project, again confirmed this accurate prediction of CRM in general setting, with an $82 \%$ agreement between MRI and histopathology for the prediction of tumor extent into the mesorectal fat ${ }^{16}$. 
Chapter 2

So far, the role of $C T$ for local staging has been investigated in a Dutch multicenter study (SPICTRE study) of which we are awaiting the definitive results. Preliminary results of this study however show the ability of multislice spiral CT to accurately predict a negative CRM in high located rectal cancer but its inability to accurately predict the margins in low rectal tumors. When MRI is not available CT could therefore be used as an alternative to select the low risk patients with high rectal tumors and wide margins from the high risk patients who preferably need additional MRI.

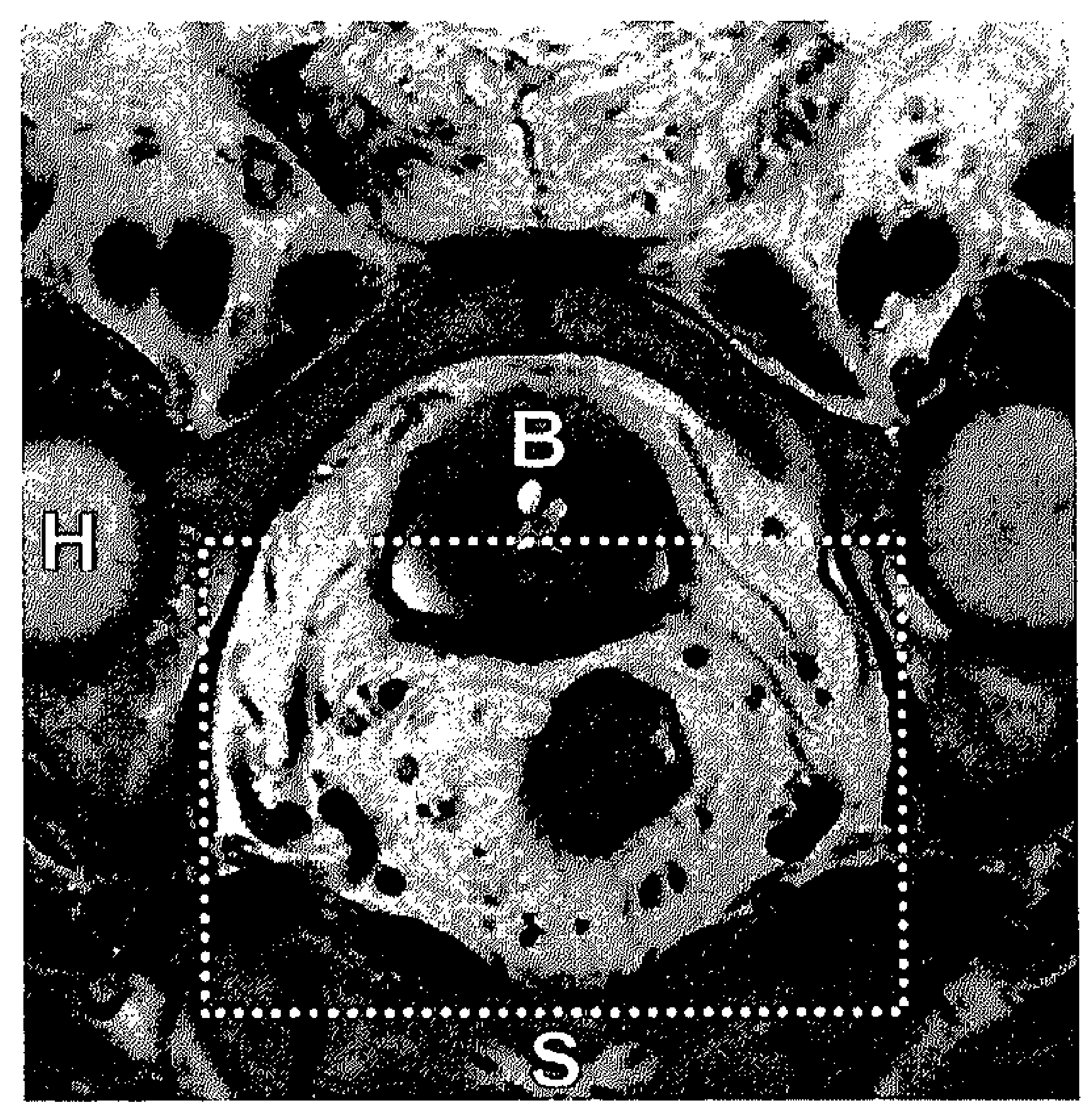

Figure 2.2a Axial T2-weighted fast spin-echo (TR/TE 3427/150 $\mathrm{ms}$ ) MR image: overview of the pelvis. $B=$ bladder, $H=$ head of femur, $S=$ sacrum

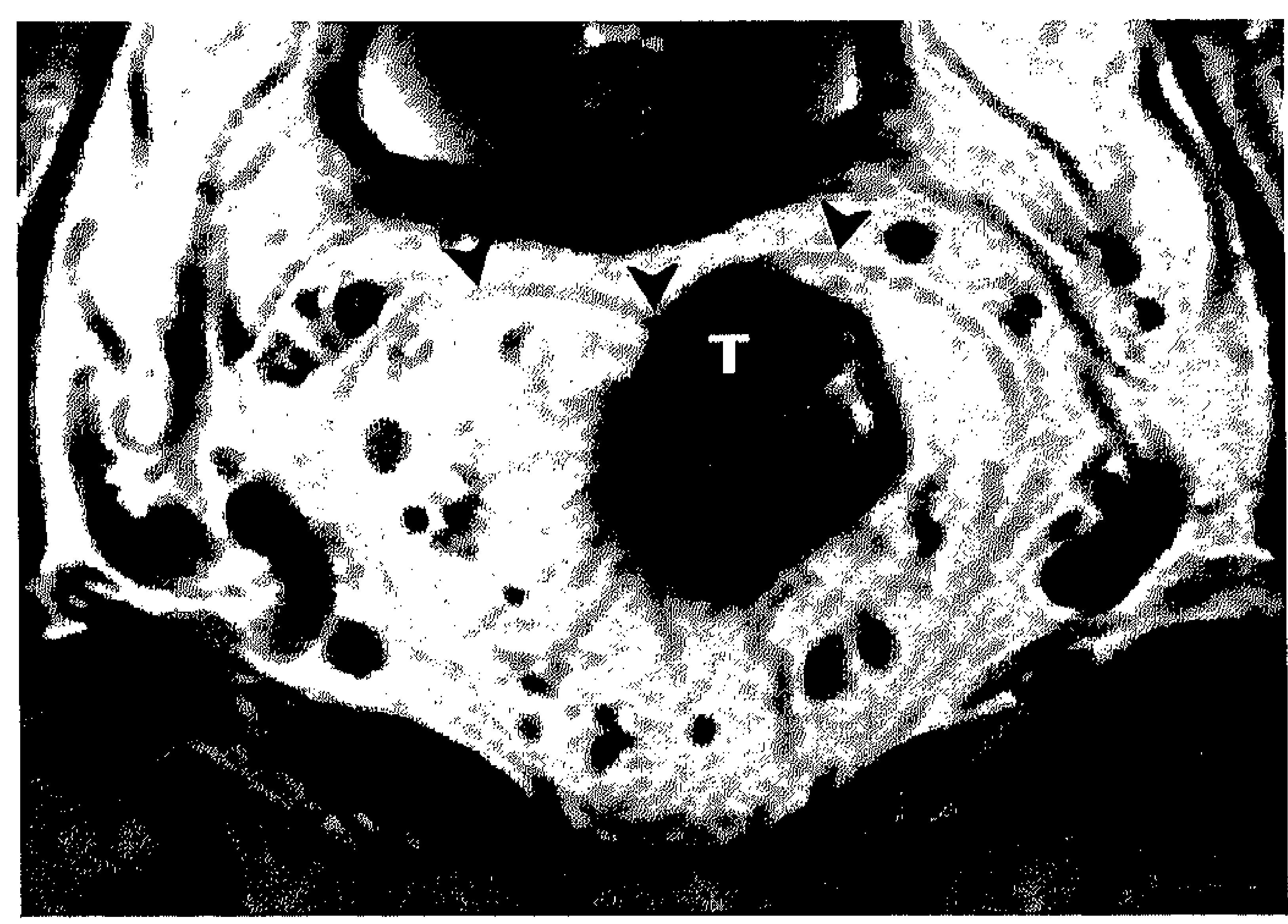

Figure 2.26 Detailed image of selection as indicated in figure $2.2 a$ shows a $T_{3}$ rectal tumor $(T)$ retracting the mesorectal fascia (black arrow heads), therefore an involved circumferential resection margin 
An additional advantage of MRI -with its accurate prediction of CRM involvement and organ invasion - is that it can serve as an important tool for the surgeon and radiotherapist to plan optimal treatment, especially for the large tumors. Ideally, surgeons should perform tumor resection while viewing MR images at the operating theater, in order to obtain a complete resection.

Prediction of nodal involvement has always been a challenge in imaging. Planar imaging modalities such as CT only rely on size and shape criteria. As has already been described by Dworak et al in 1989, a substantial percentage of patients with positive nodal status ( $32 \%$ ) only has nodes smaller than $5 \mathrm{~mm}$ and there are even cases in which positive nodes are smaller than $2 \mathrm{~mm}(8 \%)^{17}$. Therefore, when relying on size and shape only, sensitivity for detection of nodal involvement is low (MRI, CT: 66\%, 55\% resp) ${ }^{18}$. In the meta-analysis by Lahaye et $\mathrm{al}^{15}$ the accuracy of EUS is slightly, but not significantly better than CT and MRI for the prediction of nodal status. This is probably related to the fact that EUS used echo texture and border criteria in addition to size and shape criteria used by CT and MRI. However, none of these three imaging modalities can be reliably used for clinical decision-making.

With the use of USPIO (Ultrasmall Paramagnetic Iron Oxide) contrast-enhanced MRI, promising results for prediction of nodal status have been reported. USPIOMRI can be performed at 1.5 T, making it of use in general clinical practice. USPIO-MRI has been studied in bladder/prostate cancer, head/neck cancer and gynecologic malignancies in single center settings ${ }^{19-23}$. Will et al showed in a recent meta-analysis an overall sensitivity and specificity of 0.88 and 0.96 for the prediction of nodal status in different malignancies ${ }^{24}$. Only one study investigated USPIO-MRI in rectal cancer. This study by Koh et al ${ }^{23}$ showed promising results, but accuracy of USPIO-MRI and value for clinical decisionmaking needs to be further examined in routine setting.

\section{DISTANT STAGING}

The classical imaging technique for the detection of liver metastases is transabdominal ultrasound. Transabdominal ultrasound is inexpensive and widely available. However, it has a sensitivity for detection of liver metastases of 


\section{Chapter 2}

only $40-69 \%$ and is therefore an unreliable tool for screening in a high prevalence group. Multislice Spiral CT (MSCT) has a sensitivity for detection of liver metastases of $65-85^{25-28}$, making it the preferred method, after MRI with liver specific contrast agents.

SPIO contrast enhanced MRI has a high sensitivity ( 67-100\%) and specificity (89$100 \%)$ for detection and characterization of liver lesions ${ }^{26,29-31}$, and is therefore the most accurate tool for evaluation for liver metastases, apart from the more invasive Intra Operative US. Because MRI is more expensive and less widely available than $C T$ it is not considered as a practical routine screening tool.

So far the value of FDG-PET for detection of metastases has mainly been reported for the detection of recurrent liver metastases after curative resection ${ }^{32}$, and there are only few studies on the detection of primary liver metastases. Antoch et al show that PET equals MRI for the detection of liver metastases ${ }^{33}$, however to date, there is no evidence that PET would do better than $\mathrm{MSCT}^{34}$. The additional role of PET however is clearly recognized in the detection of extrahepatic metastatic disease (bone, supraclavicular, peritoneal) ${ }^{35}$.

24 Chest $\mathrm{CT}$ is obviously more sensitive for detection of pulmonary nodules than is chest X-ray. The low specificity (20-74\%) of chest CT in patients with negative chest $X$-ray still remains a problem, leading to many false-positive finding $s^{36,37}$. As a well-designed, prospective comparative study between chest $\mathrm{x}$-ray and chest $C T$ in patients with rectal cancer is lacking, it remains debatable whether routine chest $C T$ should be performed for screening in all patients with primary rectal cancer. From a practical point of view, when one chooses to perform a staging CT of the liver, it is only a small step to combine this with a CT chest. Whether one chooses to stage all patients or those with a higher prevalence of metastatic disease, i.e. stage III patients, is a matter of cost efficiency.

\section{CONCLUSIONS}

Preoperative imaging in rectal cancer is important for accurate staging and stratification into different risk groups. This allows a differentiated neoadjuvant treatment. For the selection of superficial tumors that can be treated with local excision, EUS is the optimal modality. For all other rectal cancers, MRI is 
recommended in the preoperative work up, because MRI has been proven to be the most accurate modality for prediction of an involved CRM. For accurate prediction of the nodal status it remains to be seen whether contrast agents like USPIO will become successful.

Distant staging is important as it selects patients where the focus should be more on systemic than on locoregional treatment. CT chest and abdomen is recommended as a baseline staging. 


\section{REFERENCES}

1.

3.

5.

7.

8.

9.

10.

11. Mentha G, Majno PE, Andres A, Rubbia-Brandt L, Morel P, Roth AD. Neoadjuvant chemotherapy and

Sagar PM, Pemberton JH. Surgical management of locally recurrent rectal cancer. $\mathrm{Br} J$ Surg 1996;83(3): 293-304.

Heald RJ, Ryall RD. Recurrence and survival after total mesorectal excision for rectal cancer. Lancet $1986 ; 1(8496): 1479-1482$.

Saver R, Becker H, Hohenberger W, Rodel C, Wittekind C, Fietkau R, Martus P, Tschmelitsch J, Hager $E$, Hess $C F$, Karstens $J H$, Liersch $T$, Schmidberger $H$, Raab $R$. Preoperative versus postoperative chemoradiotherapy for rectal cancer. N Engl J Med 2004;351(17): 1731-1740.

Beets-Tan RG, Beets GL. Rectal cancer: review with emphasis on MR imaging. Radiology 2004;232(2): $335-346$.

Greene F, Balch C, Page D. AJCC Cancer Staging Manual (6th edn). Springer-Verlag: New York, 2002. Nagtegaal ID, Marijnen CA, Kranenbarg EK, van de Velde CJ, van Krieken JH. Circumferential margin involvement is still an important predictor of local recurrence in rectal carcinoma: not one millimeter but two millimeters is the limit. Am J Surg Pathol 2002;26(3): 350-357.

Steup WH, Moriya $Y$, van de Velde CJ. Patterns of lymphatic spread in rectal cancer. A topographical analysis on lymph node metastases. Eur J Cancer 2002;38(7): 911-918.

. Shah SA, Haddad R, Al-Sukhni W, Kim RD, Greig PD, Grant DR, Taylor BR, Langer B, Gallinger S, Wei AC. Surgical resection of hepatic and pulmonary metastases from colorectal carcinoma. J Am Coll Surg 2006;202(3): 468-475.

Manfredi S, Lepage C, Hatem C, Coatmeur O, Faivre J, Bouvier AM. Epidemiology and management of liver metastases from colorectal cancer. Ann Surg 2006;244(2): 254-259.

Figueredo A, Rumble RB, Maroun J, Earle CC, Cummings B, MCLeod R, Zuraw L, Zwaal C. Follow-up of patients with curatively resected colorectal cancer: a practice guideline. BMC Cancer 2003;3: 26. resection of advanced synchronous liver metastases before treatment of the colorectal primary. $B r J$ Surg 2006;93(7): 872-878.

12. Beets-Tan RG, Beets GL, Borstlap AC, Oei TK, Teune TM, von Meyenfeldt MF, van Engelshoven JM. Preoperative assessment of local tumor extent in advanced rectal cancer: CT or high-resolution MRI? Abdom /maging 2000;25(5): 533-541.

13. Beets-Tan RG, Beets GL, Vliegen RF, Kessels AG, Van Boven $H$, De Bruine $A$, von Meyenfeldt MF, Baeten $C G$, van Engelshoven JM. Accuracy of magnetic resonance imaging in prediction of tumourfree resection margin in rectal cancer surgery. Lancet 2001;357(9255): 497-504.

14. Poon FW, McDonald A, Anderson JH, Duthie F, Rodger C, McCurrach G, McKee RF, Horgan PG, Foulis $A K$, Chong D, Finlay IG. Accuracy of thin section magnetic resonance using phased-array pelvic coil in predicting the T-staging of rectal cancer. Eur J Radiol 2005i53(2): 256-262.

15. Lahaye MJ, Engelen SM, Nelemans PJ, Beets GL, van de Velde CJ, van Engelshoven JM, Beets-Tan RG. Imaging for predicting the risk factors--the circumferential resection margin and nodal disease-of local recurrence in rectal cancer: a meta-analysis. Semin Ultrasound CTMR 2005;26(4): 259-268.

16. Brown G, Daniels IR, Norman A. MRI Predicts Surgical Resection Margin Status in Patients with Rectal Cancer: Results from the MERCURY Study Group. In: RSNA 2004. Chicago; 2004.

17. Dworak O. Number and size of lymph nodes and node metastases in rectal carcinomas. Surg Endosc 1989;3(2): $96-99$.

18. Bipat $S$, Glas AS, Slors FJ, Zwinderman AH, Bossuyt PM, Stoker J. Rectal cancer: local staging and assessment of lymph node involvement with endoluminal US, CT, and MR imaging--a meta-analysis. Radiology 2004;232(3): 773-783.

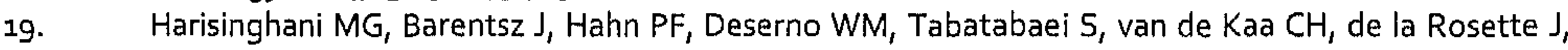
Weissleder R. Noninvasive detection of clinically occult lymph-node metastases in prostate cancer. $N$ Engl J Med 2003;348(25): 2491-2499.

20. Bellin MF, Roy C, Kinkel K, Thoumas D, Zaim S, Vanel D, Tuchmann C, Richard F, Jacqmin D, Delcourt $A_{\text {, Challier }} E_{\text {, Lebret }} T$, Cluzel P. Lymph node metastases: safety and effectiveness of MR imaging with ultrasmall superparamagnetic iron oxide particles--initial clinical experience. Radiology 1998;207(3): 799-808. 

Depondt $M$, Mattelaer $C$, Petit $P$, Champsaur $P$, Riehm S, Dadashitazehozi $Y$, de Jaegere $T$, Marchal $G$, Chevalier D, Lemaitre L, Kubiak $C$, Helmberger $R$, Halimi P. Lymph node metastases from head and neck squamous cell carcinoma: MR imaging with ultrasmall superparamagnetic iron oxide particles (Sinerem MR) -- results of a phase-III multicenter clinical trial. Eur Radiol 2002;12(5): 11041113.

22. Keller TM, Michel SC, Frohlich J, Fink D, Caduff R, Marincek B, Kubik-Huch RA. USPIO-enhanced MRI for preoperative staging of gynecological pelvic tumors: preliminary results. Eur Radiol 2004;14(6): 937-944.

23. Koh DM, Brown G, Temple L, Raja A, Toomey P, Bett N, Norman AR, Husband JE. Rectal cancer: mesorectal lymph nodes at MR imaging with USPIO versus histopathologic findings-initial observations. Radiology 2004;231(1): 91-99.

24. Will O, Purkayastha S, Chan C, Athanasiou T, Darzi AW, Gedroyc W, Tekkis PP. Diagnostic precision of nanoparticle-enhanced MRI for lymph-node metastases: a meta-analysis. Lancet Oncol 2006; $(1)$ : 52-60.

25. Bipat $S$, van Leeuwen MS, Comans EF, Pijl ME, Bossuyt PM, Zwinderman AH, Stoker J. Colorectal liver metastases: CT, MR imaging, and PET for diagnosis--meta-analysis. Radiology 2005i237(1): 123131.

26. Kinkel K, LU Y, Both $M$, Warren RS, Thoeni RF. Detection of hepatic metastases from cancers of the gastrointestinal tract by using noninvasive imaging methods (US, CT, MR imaging, PET): a metaanalysis. Radiology 2002;224(3): 748-756.

27. Ward J, Robinson PJ, Guthrie JA, Downing S, Wilson D, Lodge JP, Prasad KR, Toogood GJ, Wyatt Jl. Liver metastases in candidates for hepatic resection: comparison of helical $C T$ and gadolinium- and SPIO-enhanced MR imaging. Radiology 2005;237(1): 170-180.

28. Vitola JV, Delbeke D, Sandler MP, Campbell MG, Powers TA, Wright JK, Chapman WC, Pinson CW. Positron emission tomography to stage suspected metastatic colorectal carcinoma to the liver. Am J Surg 1996;171(1): 21-26.

29. Oudkerk M, van den Heuvel AG, Wielopolski PA, Schmitz PI, Borel Rinkes IH, Wiggers T. Hepatic lesions: detection with ferumoxide-enhanced T1-weighted MR imaging. Radiology 1997;203(2): 449456.

30. Reimer P, Jahnke N, Fiebich M, Schima W, Deckers F, Marx C, Holzknecht N, Saini S. Hepatic lesion detection and characterization: value of nonenhanced MR imaging, superparamagnetic iron oxideenhanced MR imaging, and spiral CT-ROC analysis. Radiology 2000;217(1): 152-158.

31. Vogl TJ, Schwarz W, Blume S, Pietsch M, Shamsi K, Franz M, Lobeck H, Balzer T, del Tredici K, Neuhaus P, Felix R, Hammerstingl RM. Preoperative evaluation of malignant liver tumors: comparison of unenhanced and SPIO (Resovist)-enhanced MR imaging with biphasic CTAP and intraoperative US. Eur Radiol 2003;13(2): 262-272.

32. Langenhoff BS, Oyen WJ, Jager GI, Strijk SP, Wobbes T, Corstens FH, Ruers TJ. Efficacy of fluorine18-deoxyglucose positron emission tomography in detecting tumor recurrence after local ablative therapy for liver metastases: a prospective study. J Clin Oncol 2002;20(22); 4453-4458.

33. Antoch G, Vogt FM, Freudenberg LS, Nazaradeh F, Goehde SC, Barkhausen J, Dahmen G, Bockisch A, Debatin JF, Ruehm SG. Whole-body dual-modality PET/CT and whole-body MRI for tumor staging in oncology. Jama 2003;290(24):3199-3206.

34. Antoch $G$, Saoudi $N$, Kuehl $H$, Dahmen $G$, Mueller SP, Beyer T, Bockisch A, Debatin JF, Freudenberg LS. Accuracy of whole-body dual-modality fluorine-18-2-fluoro-2-deoxy-D-glucose positron emission tomography and computed tomography (FDG-PET/CT) for tumor staging in solid tumors: comparison with CT and PET. J Clin Oncol 2004;22(21): 4357-4368.

35. Wiering B, Krabbe PF, Jager GJ, Oyen WJ, Ruers TJ. The impact of fluor-18-deoxyglucose-positron emission tomography in the management of colorectal liver metastases. Cancer 2005;104(12): 26582670 .

36. Povoski SP, Fong Y, Sgouros SC, Kemeny NE, Downey RJ, Blumgart LH. Role of chest CT in patients with negative chest $X$-rays referred for hepatic colorectal metastases. Ann Surg Oncol 1998;5(1): 9-15. 


\section{Chapter 2}

37. Kronawitter U, Kemeny NE, Heelan R, Fata F, Fong $Y$. Evaluation of chest computed tomography in the staging of patients with potentially resectable liver metastases from colorectal carcinoma. Cancer 1999;86(2): 229-235. 


\section{Location of involved mesorectal and}

extramesorectal lymph nodes in patients with primary rectal cancer: preoperative assessment with MR imaging

S.M.E. Engelen, R.G.H. Beets-Tan, M.J. Lahaye, A.G.H. Kessels, G.L. Beets 


\section{Chapter 3}

\section{ABSTRACT}

Aim The purpose of this study is to evaluate the location of involved mesorectal and extramesorectal lymph nodes as depicted on preoperative MRI. Preoperative availability of this information might be useful for the surgeon as well as the radiation therapist and medical oncologist for optimal treatment strategy: type and extent of neoadjuvant treatment as well as extent of surgical resection. Methods Forty-one patients with biopsy-proven rectal cancer were included. All patients underwent preoperative MRI using USPIO (lymph node specific contrast agent). Location of all mesorectal and extramesorectal nodes visible on MRI was recorded, as well as USPIO prediction on nodal status. Lesion-by-lesion analysis using histology after surgery was performed for patients who did not receive long course chemoradiation therapy.

Results There were 438 nodes visible, 94 of which were malignant. Most nodes are located in the laterodorsal part of the mesorectum, with no difference in distribution between positive and negative nodes. In relation to height of tumor, the majority of positive nodes are located at tumor height or above. There were significantly more negative nodes $(9.6 \%)$ located below tumor height as compared to positive nodes (2.1\%). There were 40 extramesorectal nodes, in 16 patients, 5 of which were positive in 4 patients. All patients had distal rectal cancer.

Conclusion In conclusion, positive mesorectal nodes are located in the laterodorsal part of the mesorectum, at tumor height or above. Positive nodes distal to the tumor are rare, and occur in patients with more proximal nodal metastases. Positive extramesorectal nodes mainly occur in patients with distal rectal cancer with nodal metastases in the mesorectum. 


\section{INTRODUCTION}

Nodal involvement is one of the factors associated with a worse prognosis in patients with rectal cancer ${ }^{1}$. Previous research has already shown the patterns of nodal involvement in rectal cancer. The earliest studies mainly focussed on the upward spread along the ascending branch of the inferior mesenteric vein and the downward spread towards the perineum and inguinal lymph nodes ${ }^{2-4}$. Following studies drew attention to the incidence of lateral lymphatic spread along the middle rectal artery, outside the mesorectum ${ }^{5}$, discussing the option of lateral nodal dissections ${ }^{6-11}$. The distribution of lymph nodes within the mesorectum has been studied by several investigators ${ }^{12-16}$. Topor et a $\left.\right|^{12}$ described the distribution of mesorectal nodes in a cadaver study of healthy volunteers. They concluded that the majority of mesorectal nodes are located in the upperposterior part of the mesorectum. Wang et $\mathrm{al}^{14-16}$ reported several studies on size and location of involved mesorectal lymph nodes. They concluded that a substantial part of mesorectal tumor nodes are smaller than $5 \mathrm{~mm}$ and that involved mesorectal nodes occur more often ipsilateral to the tumor than contralateral, with substantial number of nodes in the outer layer of the mesorectum.

Generally the information on the extent and location of involved nodes is only available after surgical resection, as the common imaging modalities lack accuracy for a reliable preoperative assessment of nodal status ${ }^{17}$. This information could however be useful in planning the optimal treatment: the type and extent of neoadjuvant treatment (e.g. adjustment of radiation fields) and the extent of the resection of mesorectal and extramesorectal lymphatic compartments. With modern MR techniques and lymph node specific contrast agents such as USPIO (Ultra small Paramagnetic Iron Oxide), the accuracy for nodal staging has improved in bladder and prostate as well as head and neck cancer $^{18}$. Also for rectal cancer, nodal staging is promising ${ }^{19}$. The distribution of involved nodes in rectal cancer however, has not yet been thoroughly evaluated. The aim of this study is to describe the distribution of involved mesorectal and extramesorectal lymph nodes as depicted on MRI in patients with primary rectal cancer. 


\section{Chapter 3}

\section{METHODS}

\section{Patients}

Forty-one patients with biopsy-proven primary rectal cancer were included at the University Hospital Maastricht, Maastricht, The Netherlands, between May 2004 and June 2006. Twenty-eight patients were male, mean age at diagnosis was 69.1 $+/-10.3$ years. Institutional review board approval and informed consent were obtained.

USPIO-MRI

All patients received a pelvic MRI using Ultrasmall Super Paramagnetic Iron Oxide (a lymph node specific contrast agent) preoperatively. The USPIO MR contrast agent (Sinerem ${ }^{\infty}$, Guerbet Laboratories, Roissy, France) consists of low molecular weight iron oxide coated with dextran. Sinerem ${ }^{\circledR}$ was supplied as a powder which was reconstituted using saline and administered at a dose of $2.6 \mathrm{mg} \mathrm{Fe} / \mathrm{kg}$. Administration took place by slow intravenous infusion within a period of 45 minutes and was completed twenty-four to thirty-six hours before MRI scan. Imaging was performed on a 1.5 T Gyroscan (Philips Medical Systems, Best, The Netherlands). Sequences used were sagittal and axial 2D T2W TSE, axial $3 D$ $T_{2} * W$ GRE and axial 3D TIW GRE. Patients did not receive bowel or other preparation. Total scan time was approximately 40 minutes.

\section{Image evaluation}

An expert MR radiologist measured height and size (length) of tumor on sagittal images. All intra- and extramesorectal lymph nodes visible on pelvic USPIO MRI were prospectively recorded on an anatomical road map to make accurate lesionby-lesion analysis possible. For mesorectal nodes, the following characteristics were studied after the prediction whether the node was positive or negative for tumor invasion based on USPIO criteria ${ }^{19}$ : size of node ( $\mathrm{mm}$ long axis), position of node in relation to tumor in axial section through the mesorectum (figure 3.1), position of node in craniocaudal relation to the tumor (at same height as tumor, more distal or more proximal) (figure 3.2), distance tumor-node in axial and sagittal plane $(\mathrm{mm})$. Extramesorectal nodes were also predicted to be positive or negative for tumor invasion, size was measured and the location of these nodes 
in the anatomical spaces were defined: left or right internal iliac, obturator or external iliac space.

\section{Stage and treatment}

There were 10, 11, 15 and 5 patients with stage I - IV (see table 1.1, general introduction) rectal cancer respectively. The treatment decision was based on MR images. Patients without involvement of the mesorectal fascia and without massive nodal involvement $\left(\mathrm{N}_{2}\right)$ underwent a short course radiotherapy ( $5 \times 5 \mathrm{~Gy}$ ) prior to TME surgery $(n=23)$. Patients with locally advanced disease (predicted involvement of the mesorectal fascia or massive nodal involvement) received a long course of chemo-radiotherapy (capecitabine/oxaliplatin and 28x1.8 Gy) followed by resection $(n=18)$. Twenty-two patients underwent low anterior resection, 11 patients an abdominoperineal resection and 8 patients did not receive surgical treatment ( 7 because of inoperable local or distant disease; 1 patient refused surgery).

\section{Histopathological evaluation}

The resection specimen was evaluated in a standardized way: (1) the specimen was fixed in formalin for $24-48$ hrs, (2) the circumferential resection plane was inked, (3) the specimen was sliced transversely every $5 \mathrm{~mm}$, to search for and accurately determine location of nodes, (4) the nodes were stained with Haematoxylin Eosin-staining and evaluated using standard microscopic investigation. For patients who underwent TME surgery after $5 \times 5$ Gy radiotherapy, nodes on MRI were correlated to nodes found at histology in a lesion-by-lesion analysis. As reported by Marijnen et al. $5 \times 5 \mathrm{~Gy}$ radiation therapy followed by immediate surgery does not sterilize nodes ${ }^{20}$, and a lesion-by-lesion analysis of the nodes in these patients is therefore justified. Only nodes seen on MRI as well as found at histology were taken into account.

Despite the fact that a lesion by lesion analysis is not reliable after chemoradiation it was decided to include the patients with locally advanced tumors in order to increase the number of positive nodes. Only including the relatively small tumors with a small number of involved nodes would produce less solid information on the location of involved nodes. However because of chemoradiation effects, there was no histological Gold Standard. For these 


\section{Chapter 3}

patients, MR prediction of nodal status per node was used as the reference standard for the analysis. Negative predictive value (NPV) of USPIO-MRI is high, $98 \%$ from our own data, at the expense of some false-positives ${ }^{19}$. Therefore we realize that although the negative nodes are truly predicted negative, there will be some negative nodes overstaged on USPIO MRI in this group.

\section{Statistical analysis}

Linear regression was used to test for difference in size of the node and in absolute distance from tumor to node in axial and cranio-caudal plane between the positive and negative nodes. For difference between positive and negative nodes in height of node in relation to tumor (distal vs other levels), logistic regression was used and for node in relation to tumor in axial plane multinominal logistic regression was used. Correlated data within one patient was taken into account with the cluster option in STATA Software (Stata Statistical Software: Release 9. College Station, TX: StataCorp LP). P-values $<0.05$ were considered statistically significant.

\section{RESULTS}

\section{Mesorectal nodes}

There were 438 mesorectal nodes found in 41 patients. Twenty patients had 94 positive mesorectal nodes. Size distribution of positive as well as negative nodes is shown in table 3.1. Positive nodes generally are larger than negative nodes $(p<0.001)$, but there is still a substantial percentage of positive nodes smaller than $5 \mathrm{~mm}$.

\begin{tabular}{|c|c|c|}
\hline \multirow[b]{2}{*}{ Size of mesorectal node } & \multicolumn{2}{|c|}{$\mathrm{N}$} \\
\hline & Negative nodes & Positive nodes \\
\hline$<5 \mathrm{~mm}$ & 224 & 15 \\
\hline $5-10 \mathrm{~mm}$ & 117 & 69 \\
\hline$>10 \mathrm{~mm}$ & 3 & 10 \\
\hline Total & 344 & 94 \\
\hline
\end{tabular}

Generally, the distribution pattern of involved mesorectal lymph nodes follows the distribution of nodes overall, which are located in the laterodorsal part of the 
mesorectum, at same height as the tumor or above (see figure 3.1 and table 3.2, figure 3.2 and table 3.3 and figure $3.3 \& 3.4$ ). The only difference in distribution between positive and negative nodes is found for the nodes located below the tumor.

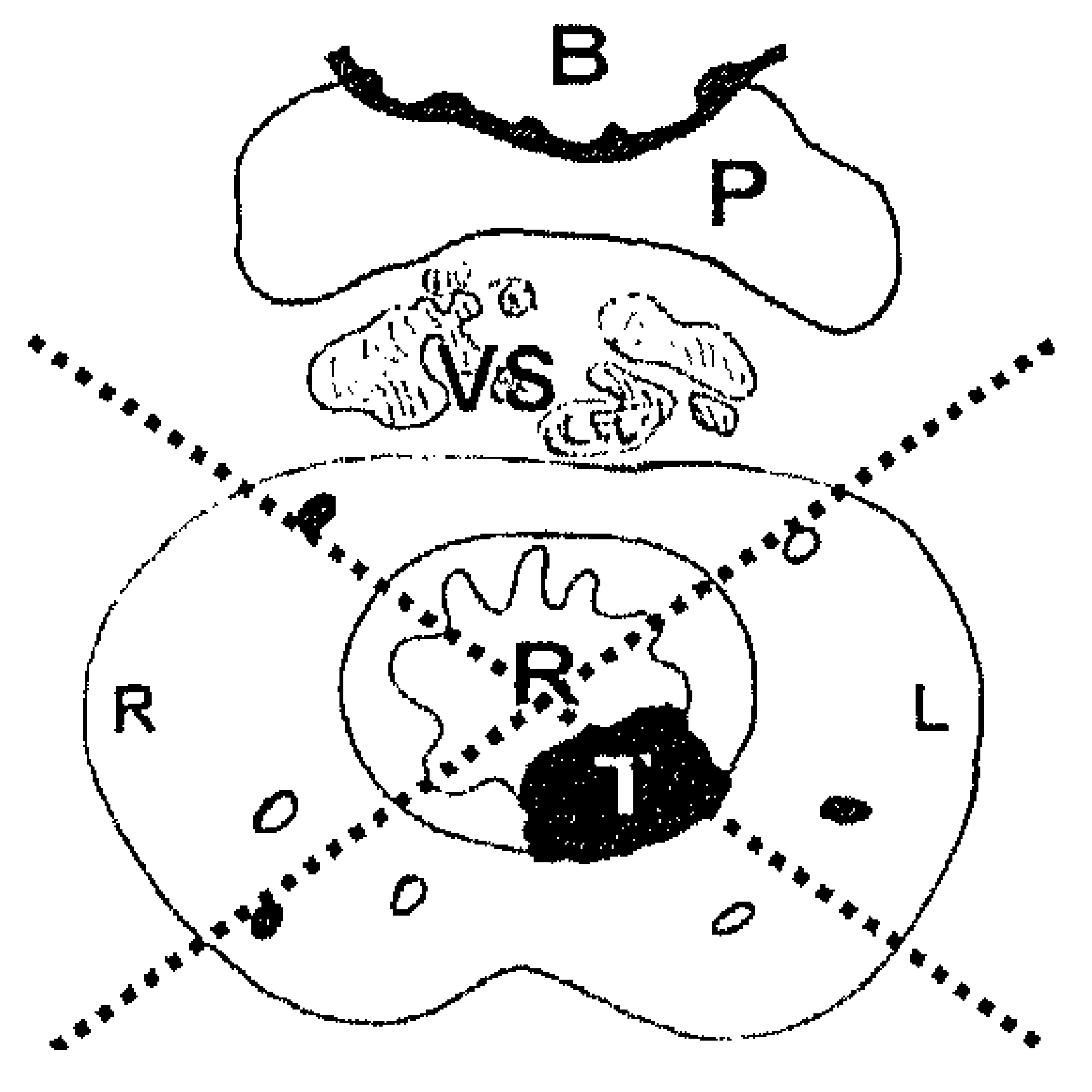

Figure 3.1 Schematic drawing in axial section through the mesorectum. Lymph nodes are localized in anterior, left-lateral, dorsal or right-lateral compartment. $(B=$ bladder, $P=$ prostate, $V S=$ seminal vesicles, $R=$ rectum, $T=$ rectal tumor)

and $14 \mathrm{~mm}$ below the distal border of the tumor. In both patients, there were numerous other malignant nodes located at tumor height.

\begin{tabular}{lcc}
\hline Location of mesorectal node & $\mathrm{N}$ & \\
in axial plane & Negative nodes & Positive nodes \\
\hline Left lateral & 116 & 28 \\
Dorsal & 122 & 45 \\
Right lateral & 90 & 20 \\
Ventral & 16 & 1 \\
Total & 344 & 94 \\
\hline Table 3.2 Distribution of positive and negative mesorectal nodes in axial plane through the mesorectum.
\end{tabular}

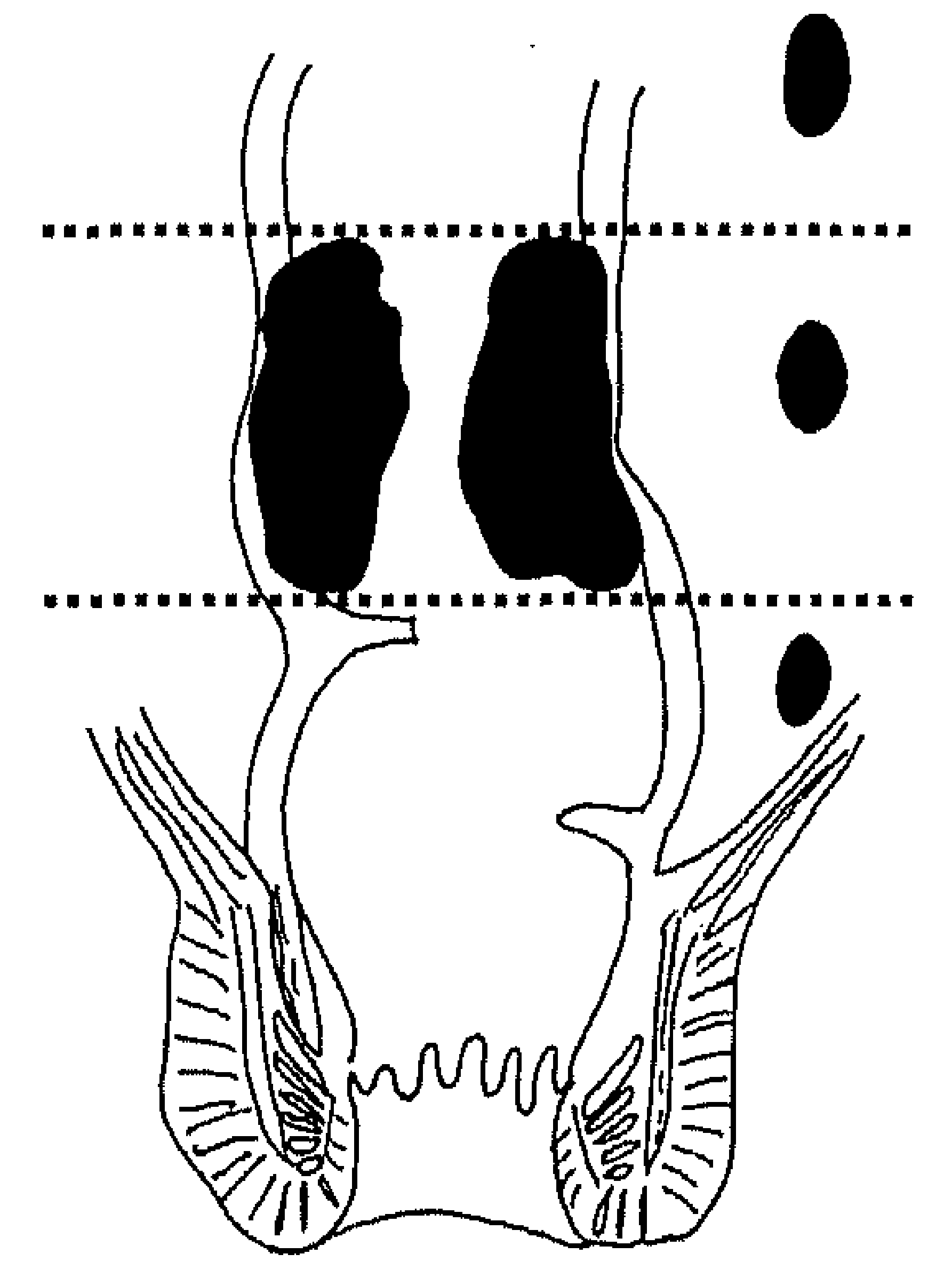

Figure 3.2 Schematic drawing of sagittal plane through the rectum. Lymph nodes are localized at same height, proximal or distal to the tumor

\section{Extramesorectal nodes}

There were 40 extramesorectal nodes found in 16 patients (range: 1-6 per patient). In 4 patients, there were 5 positive extramesorectal nodes. All 4 patients had distal rectal cancer, located $1-2 \mathrm{~cm}$ from the anal verge. Positive extramesorectal nodes were located at the root of the middle rectal 


\section{Chanter 3}

artery in the internal iliac space (3 nodes) (see figure 3.5) or in the obturator space ( 2 nodes).

\begin{tabular}{|c|c|c|}
\hline \multirow[b]{2}{*}{ Height of mesorectal node } & \multicolumn{2}{|c|}{$\mathbf{N}$} \\
\hline & Negative nodes & Positive nodes \\
\hline Proximal to tumor & 91 & 30 \\
\hline Same height & 220 & 62 \\
\hline Distal to tumor & 33 & 2 \\
\hline Total & 344 & 94 \\
\hline
\end{tabular}

Table 3.3 Distribution of positive and negative nodes in sagittal plane in relation to the tumor: at same height, more proximal or more distal

In 3 out of 4 patients with positive extramesorectal nodes, there were involved mesorectal nodes as well. Further evaluation of the remaining single patient with an involved lateral node without mesorectal nodes revealed a synchronous primary tumor of the bladder which explained the exceptional distribution. Most likely, this positive extramesorectal node was a metastasis of the bladder carcinoma rather than due to the rectal tumor.

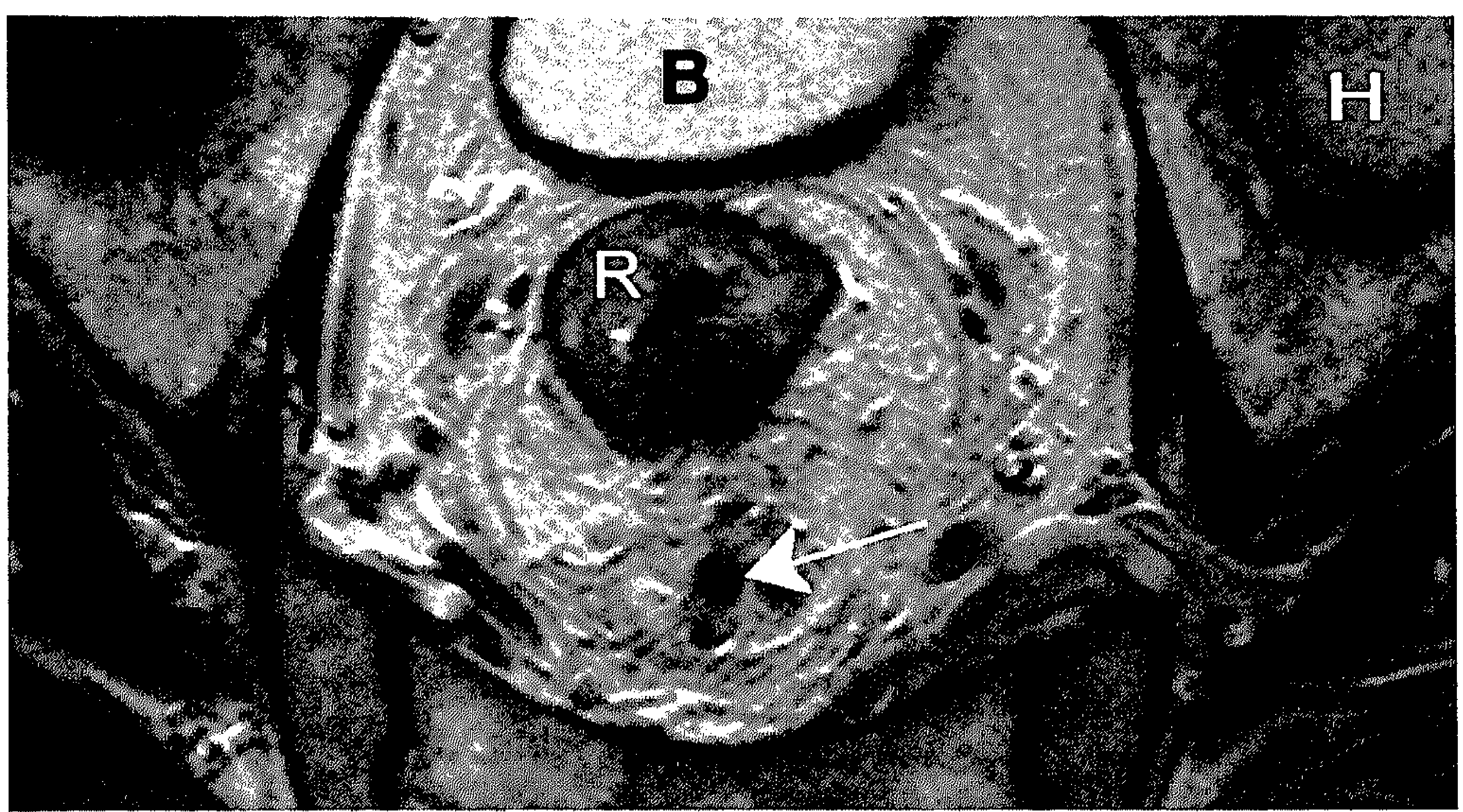

Figure 3.3 Axial T2-weighted fast spin-echo (TR/TE 3427/150 ms) MR image shows the rectum with a rectal tumor $(R)$. At the height of the tumor, there is a lymph node located in the dorsal compartment of the mesorectum (white arrow). $B=$ bladder, $H=$ head of fermur 


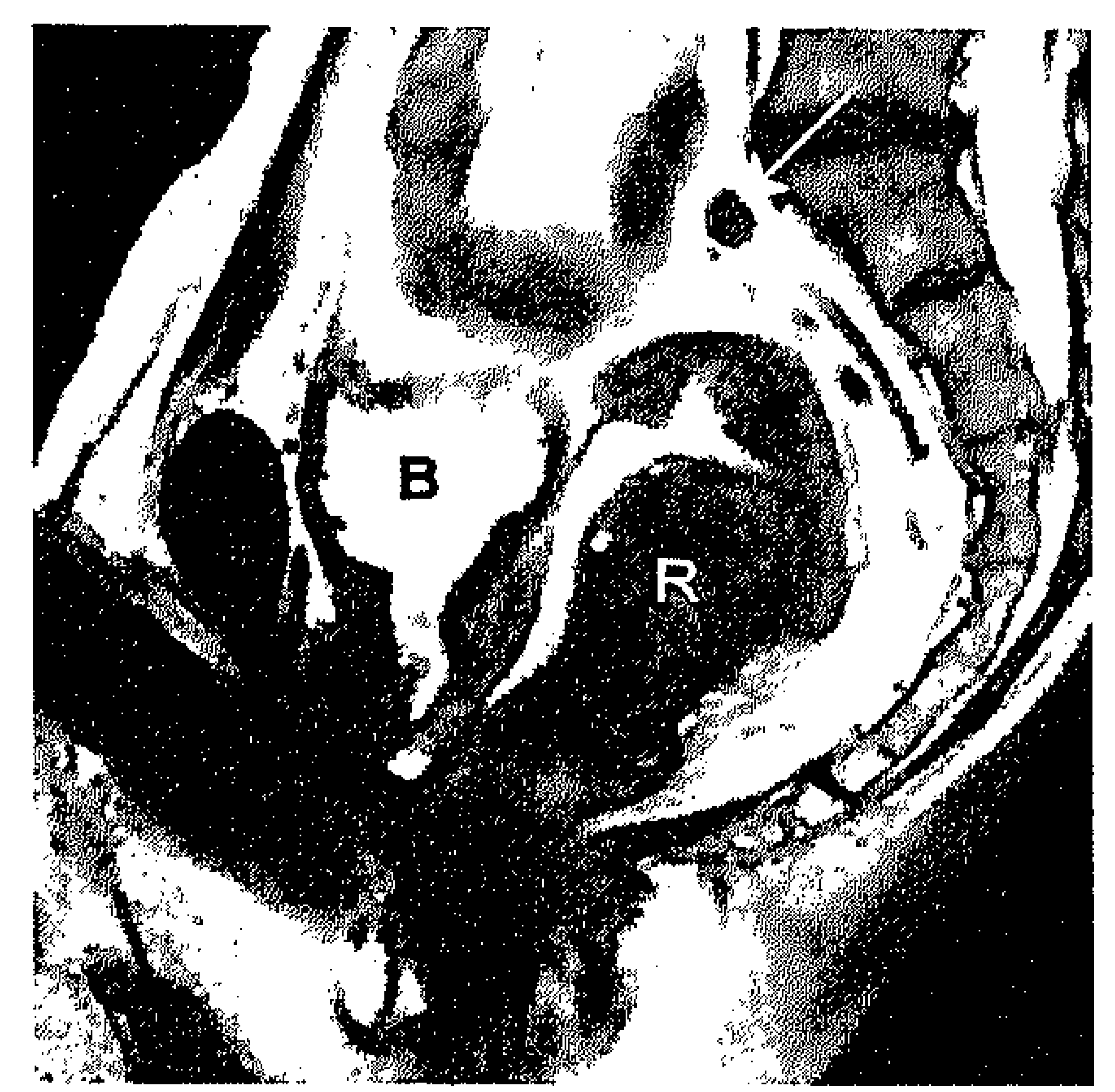

Figure 3.4 Sagittal T2-weighted fast spin-echo (TR/TE 3427/150 ms) MR image shows the rectum with a rectal tumor ( $R$ ). Proximal to the tumor, there is a lymph node located in the dorsal compartment of the mesorectum (white arrow). $B=$ bladder

\section{DISCUSSION}

The results of this study on the location of (extra)mesorectal nodes as depicted on preoperative MRI show that the majority of involved mesorectal nodes is located at tumor height or above. Generally, the location of the involved mesorectal nodes follows the location of mesorectal nodes overall, with one exception: there are significantly less positive nodes distal to the tumor as compared to negative nodes, with in our series no positive nodes lower than 14 $\mathrm{mm}$ below tumor level. Furthermore, when seen in axial plane, most nodes are located in the dorsal and lateral parts of the mesorectum. Results of this study are a confirmation of earlier histopathology studies ${ }^{12-16}$. Also the results on the extramesorectal nodes (very few positive nodes; in patients with distal rectal cancer) are in line with earlier, mostly Japanese studies, in which a lateral lymphadenectomy was performed routinely ${ }^{8,}{ }^{10,21}$ : overall a low incidence of involved extramesorectal nodes, most often in patients with distal rectal cancer with mesorectal nodal involvement.

However, in contrast to histopathology studies performed earlier ${ }^{12-15}$, this study shows that this information can be available preoperatively. The preoperative knowledge on the location of involved nodes might be important for multidisciplinary rectal cancer treatment. The neoadjuvant treatment plan could be changed (dose, schedule, radiation fields), for instance in case of massive 
mesorectal nodal unvolvement, or suspected malignant lateral nodes, a feature that is considered a representation of distant disease according to the TNM classification ${ }^{2 .}$. For the surgeon, this preoperative knowledge could be used to decide on the extent of the distal mesorectal resection. With regard to patients with malignant lateral nodes, there is the question whether or not to perform a lateral lymphadenectomy after neoadjwant treatment. Although often advocated by Japanese studies ${ }^{1 / 1}$, western surgeons are reluctant to perform lateral lymphadenectomy because of the urogenital morbidity.

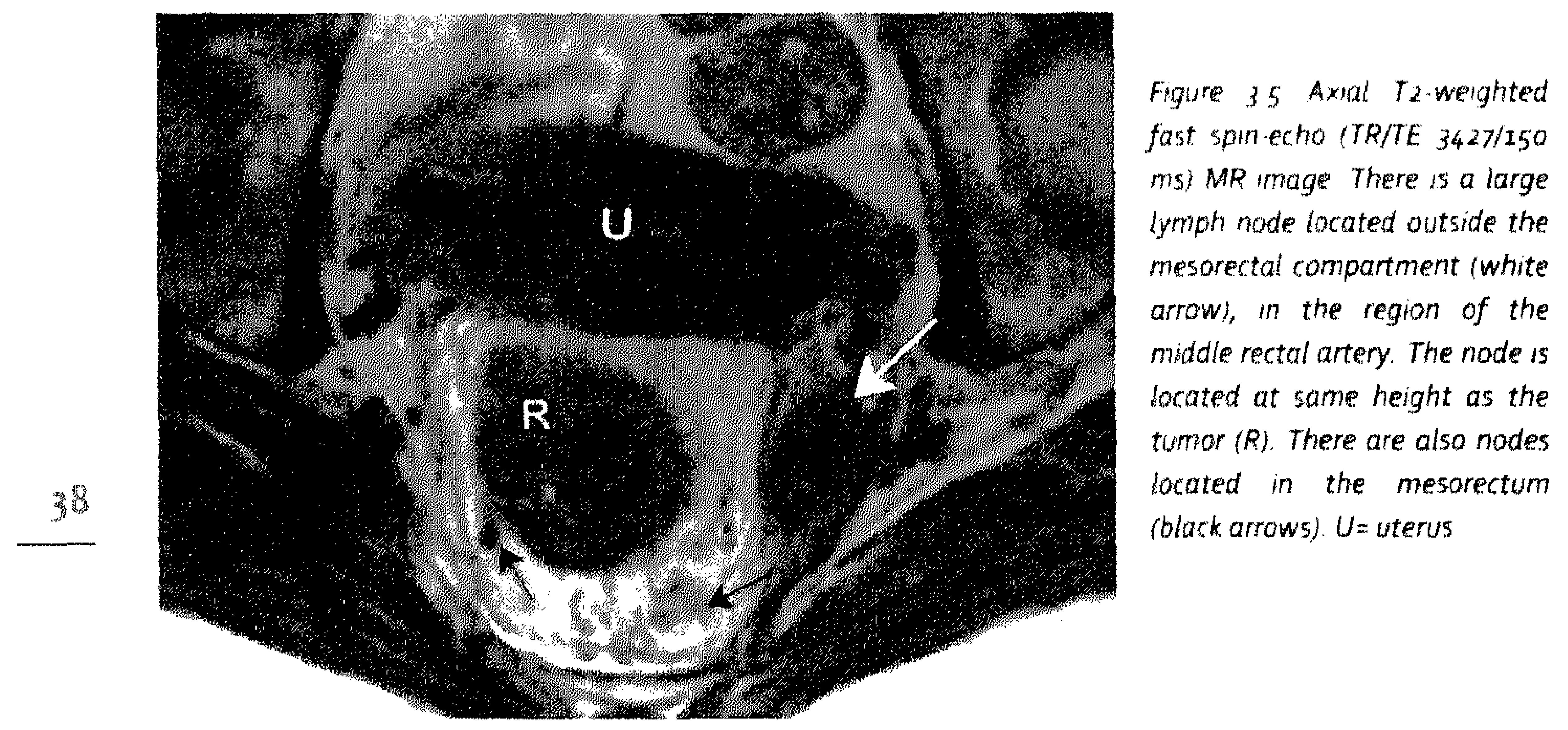

Knowledge of nodal distribution in rectal cancer might also be of help for a radiologist in his prediction of nodal metastases. Criteria of size, shape, border and pattern are usually used in classical unenhanced MRI to distinguish between benign and malignant nodes ${ }^{23}$. However, the results of a recent meta-analysis show very moderate accuracy of these criteria, and there are no imaging modalities with a higher accuracy for prediction of nodal status ${ }^{17}$. USPIO-MRI, used in this study for identification of nodal involvement in patients without lesion-by-lesion analysis, is known to improve accuracy of nodal status prediction in several malignancies ${ }^{18}$ and it is currently evaluated for rectal cancer ${ }^{19}$. Preliminary data show an increased accuracy for nodal status prediction as compared to non-enhanced MRI. But as important as the MRI technique is, for the radiologist it is equally important to know the anatomy and patterns of nodal 
involvement. With respect to patterns of nodal involvement, the radiologist should keep in mind that the most common location is inside the mesorectum at the level of the tumor, and above the tumor. Involved nodes distal of the tumor are rare, and occur in patients with more proximal involved nodes, and involved extramesorectal locations are equally rare and occur mostly in more advanced distal rectal cancer.

The drawback of this study is that there is no lesion-by-lesion analysis for all patients. Those patients receiving $5 \times 5$ Gy preoperative radiation therapy were included in a lesion-by-lesion analysis, whereas those patients receiving long course chemoradiation therapy preoperatively were not, as neoadjuvant therapy is known to downstage or sterilize nodes. USPIO-MRI has an extremely high negative predictive value, but there might be some false-positive nodes in our analysis due to lack of lesion-by-lesion analysis in some patients.

Furthermore, one should be aware of the fact that only lymph nodes have been studied. As shown by Wang et $\mathrm{al}^{16}$, there are isolated tumor cells or clusters of tumor cells in the mesorectal fat outside lymph nodes, which are beyond the detection level of morphological MR imaging. Wang found these tumor cells to be located down to $3.5 \mathrm{~cm}$ distal from the tumor. It is at present unknown if the pattern of spread of the microscopic nodules follows the pattern of lymphatic spread. For instance, is there more distal spread of small tumor foci than there is of involved lymph nodes? These microscopic tumor deposits might contribute to a higher rate of local recurrence when not included in the CTV (clinical target volume) of the radiation portal field or when the mesorectum is not completely resected. On this matter Wang reported distal spread of microscopic tumor deposits in $\mathrm{N}+$ patients only ${ }^{16}$. It is clear that the issue of small mesorectal deposits warrants further research.

In conclusion, the majority of involved lymph nodes in patients with primary rectal cancer, as depicted on preoperative MRI, is located at tumor height or above, in the dorsolateral compartment of the mesorectum. Involved nodes distal to the tumor are not common and occur in patients with more proximal nodal metastases. Involved extramesorectal nodes are also not common and 


\section{Chapter 3}

occur mainly in patients with distal rectal cancer with nodal metastases in the mesorectum. 


\section{Location of Lymph Nodes in Rectal Cancer}

\section{REFERENCES}

1. Kapiteijn E, Marijnen CA, Nagtegaal ID, Putter $H$, Steup WH, Wiggers T, Rutten HJ, Pahlman L, Glimelius B, van Krieken JH, Leer JW, van de Velde CJ. Preoperative radiotherapy combined with total mesorectal excision for resectable rectal cancer. N Engl J Med 2001;345(9): 638-646.

2. Miles WE. A method of performing abdominopelvic excision for carcinoma of the rectum and of the terminal portion of the pelvic colon. Lancet 1908;2: 1812-1813.

3. Gilchrist R, David V. A consideration of pathological factors influencing five-year survival in radical resection of the large bowel an rectum for carcinoma. Ann Surg 1947;126: 421-438.

4. Grinnell R. Lymphatic metatstases of carcinoma of the colon and rectum. Ann Surg 1950;131: 494506.

5. Saver I, Bacon HE. Influence of lateral spread of cancer of the rectum on radicability of operation and prognosis. Am J Surg 1951;81(1): 111-120.

6. Hojo K, Koyama $Y$, Moriya Y. Lymphatic spread and its prognostic value in patients with rectal cancer. Am J Surg 1982;144(3): 350-354.

7. Hojo K, Sawada T, Moriya Y. An analysis of survival and voiding, sexual function after wide iliopelvic lymphadenectomy in patients with carcinoma of the rectum, compared with conventional lymphadenectomy. Dis Colon Rectum 1989;32(2): 128-133.

8. Moriya $Y$, Hojo K, Sawada T, Koyama Y. Significance of lateral node dissection for advanced rectal carcinoma at or below the peritoneal reflection. Dis Colon Rectum 1989;32(4): 307-315.

9. Harnsberger JR, Vernava VM, 3rd, Longo WE. Radical abdominopelvic lymphadenectomy: historic perspective and current role in the surgical management of rectal cancer. Dis Colon Rectum 1994;37(1): 73-87.

10. Moriya Y, Sugihara K, Akasu T, Fujita S. Importance of extended lymphadenectomy with lateral node dissection for advanced lower rectal cancer. World J Surg 1997;21(7): 728-732.

11. Curti G, Maurer CA, Buchler MW. Colorectal carcinoma; is lymphadenectomy useful? Dig Surg 1998;15(3): 193-208.

12. Topor B, Acland R, Kolodko V, Galandiuk S. Mesorectal lymph nodes: their location and distribution within the mesorectum. Dis Colon Rectum 2003;46(6): 779-785.

13. Canessa CE, Badia F, Fierro S, Fiol V, Hayek $G$. Anatomic study of the lymph nodes of the mesorectum. Dis Colon Rectum 2001;44(9): 1333-1336.

14. Wang $C$, Zhou ZG, Wang $Z$, Chen DY, Zheng YC, Zhao GP. Nodal spread and micrometastasis within mesorectum. World J Gastroenterol 2005;11(23): 3586-3590.

15. Wang $C$, Zhou ZG, Wang $Z$, Li L, Zheng $Y C$, Zhao GP, Chen DY, Liu WP. Mesorectal spread and micrometastasis of rectal cancer studied with large slice technique and tissue microarray. $J$ Surg Oncol 2005i91(3): 167-172.

16. Wang $C$, Zhou $Z$, Wang $Z$, Zheng $Y$, Zhao G, $Y \cup Y$, Cheng $Z$, Chen D, Liu W. Patterns of neoplastic foci and lymph node micrometastasis within the mesorectum. Langenbecks Arch Surg 2005;390(4): 312318.

17. Lahaye MJ, Engelen SM, Nelemans PJ, Beets GL, van de Velde CJ, van Engelshoven JM, Beets-Tan RG. Imaging for predicting the risk factors-.the circumferential resection margin and nodal disease-. of local recurrence in rectal cancer: a meta-analysis. Semin Ultrasound CTMR 2005;26(4):259-268.

18. Will O, Purkayastha S, Chan C, Athanasiou T, Darzi AW, Gedroyc W, Tekkis PP. Diagnostic precision of nanoparticle-enhanced MRI for lymph-node metastases: a meta-analysis. Lancet Oncol 2006;7(1): 52-60.

Lahaye MJ, Engelen SM, Beets $G$, de Bruine $A$, von Meyenfeldt $M$, van Engelshoven JM, van de Velde $\mathrm{CH}$, Beets-Tan R. How accurate can we predict the nodal status in primary rectal cancer with USPIO MRI? Ann Oncol 2006;17 Suppl 1: i11

20. Marijnen CA, Nagtegaal ID, Klein Kranenbarg E, Hermans J, van de Velde CJ, Leer JW, van Krieken $\mathrm{JH}$. No downstaging after short-term preoperative radiotherapy in rectal cancer patient5. J Clin Oncol 2001;19(7): 1976-1984. 


\section{Chapter 3}

21. Mori T, Takahashi K, Yasuno M. Radical resection with autonomic nerve preservation and lymph node dissection techniques in lower rectal cancer surgery and its results: the impact of lateral lymph node dissection. Langenbecks Arch Surg 1998;383(6): 409-415.

22. Sobin L, Wittekind C (eds). TNM Classification of malignant tumours (6 edn). Wiley: New York, 2.002.

23. Kim JH, Beets GL, Kim MJ, Kessels AG, Beets-Tan RG. High-resolution MR imaging for nodal staging in rectal cancer: are there any criteria in addition to the size? Eur J Radiol 2004;52(1): 78-83. 


\section{USP1O MRI for nodal staging of patients with primary rectal cancer: predictive criteria}

M.J. Lahaye, S.M.E. Engelen, A.G.H. Kessels, A.P. de Bruine, M.F. von Meyenfeldt, J.M.A. van Engelshoven, C.J.H. van de Velde, G.L. Beets, R.G.H. Beets-Tan

(Radiology. 2008; 246(3):804-811.) 


\section{Chapter 4}

\section{ABSTRACT}

Aim To prospectively determine the diagnostic performance of various predictive criteria for USPIO MRI nodal staging in primary rectal cancer patients, using histology as the reference standard.

Methods Institutional review board approval and informed consent were obtained. 28 Rectal cancer patients (18 males and 10 females, mean age 68, range $54^{-89}$ years) underwent USPIO MRI with 2DT2WFSE, 3DT1WGRE and $3 \mathrm{DT}_{2}$-weighted sequences. Two observers with different reading experience evaluated each node on $3 D T 2^{*}$-weighted images for: border irregularity, short and long axis and estimated percentage of white region within the node, categorized as: $<30 \%, 30-50 \%$ or $>50 \%$. A ratio for the white region was also calculated $\left(\right.$ Ratio $\left._{A}\right)$. Signal intensity $(\mathrm{SI})$ measurements within muscle $\left(\mathrm{SI}_{\text {muscle }}\right.$ ), total node $\left(S I_{T N}\right)$, white $\left(S I_{\text {white }}\right)$ and dark $\left(S I_{\text {dark }}\right)$ region within the node were used to calculate ratios: $S I_{\mathrm{TN}} / S I_{\text {muscle }}$ and $S I_{\text {white }} / S I_{\text {dark }}$. Lesion-by-lesion analysis was performed with histology as the reference standard. Receiver operating characteristic (ROC) curves and interobserver agreement analysis were performed. The most accurate and practical criterion was then evaluated by observer 3 .

Results 236 lymph nodes in 28 patients were studied. The area under the ROCcurve (AUC) of estimated percentage of white region and Ratio ${ }_{A}$ for observer 1 and 2 were: $0.96 \& 0.99$ and $0.95 \& 0.97$, respectively. The AUC of the estimated percentage criterion for observer 3 was 0.96 . The AUC of border, short axis, long axis, $S I_{\text {TN }} /\left.S\right|_{\text {muscle }}$ and $S I_{\text {white }} / S I_{\text {dark }}$ for observer 1 were: $0.65,0.75,0.79,0.85,0.75$ and observer 2: $0.58,0.75,0.79,0.89$ and 0.79 , respectively. The interobserver agreement ( $K$ value) for the estimated white region between observer $1 \& 2,1 \& 3$, $2 \& 3$ were $0.77,0.79,0.84$, respectively. $K$ value for observer $1 \& 2$ for the border was 0.28 . The Intra Class Correlation for observer $1 \& 2$ for the short axis, long axis diameter, the Ratio $A_{A}$ the $S I_{T N} / S I_{\text {muscle }}$ and $S I_{\text {white }} / S I_{\text {dark }}$ were $0.91,0.96,0.91,0.72$ and 0.92 .

Conclusion The estimated percentage of white region and measured Ratio $\mathrm{A}_{\mathrm{A}}$ are the most accurate criteria for predicting malignant nodes with USPIO MRI, of which the first is the most practical. 
Predictive Criteria for Nodal Staging

\section{INTRODUCTION}

Rectal cancer is a common malignancy with a highly variable local recurrence rate of $3-32 \%^{1}$. In the recent past besides total mesorectal excision (TME), neoadjuvant rather than adjuvant radio(chemo)therapy is advocated to reduce the local recurrence rate ${ }^{2,3}$. Furthermore, to avoid over and undertreatment a tailor-made neoadjuvant approach for every rectal cancer patient, based on preoperative prediction of the risk for local recurrence, seems to be favorable. Important risk factors are T-stage, circumferential resection margin (CRM), nodal status and height of the tumor. Preoperative imaging is essential to identify these risk factors.

The nodal status is a well established important prognostic risk factor. The higher the number of positive nodes, the higher the risk for a local recurrence ${ }^{4 \prime}{ }^{5}$. However, prediction of the nodal status remains a problem for the radiologist. The results of a meta-analysis show that for identification of nodal disease Endoluminal Ultrasound (EUS), Magnetic Resonance Imaging (MRI) and Computed Tomography (CT) lack sufficient accuracy for clinical decision making ${ }^{6}$. Ultrasmall Superparamagnetic Iron Oxide (USPIO) MRI has been reported to be promising in differentiating benign from malignant nodes. A recent metaanalysis showed that USPIO MRI is superior to all other modalities in detection of lymph node metastases for various tumors ${ }^{7}$. Despite many promising results concerning USPIO MRI, there is still insufficient knowledge of which specific USPIO MRI criteria can be used for characterizing lymph nodes as benign or malignant. Thus, the purpose of our study was to prospectively determine the diagnostic performance of various predictive criteria for USPIO MRI nodal staging in primary rectal cancer patients, using histology as the reference standard.

\section{METHODS}

Guerbet Laboratories (Roissy, France) only provided free vials of Sinerem ${ }^{\circledR}$, that still was not commercially available at time of this study, and Guerbet Laboratories had no control of inclusion, data or the information submitted for this publication. The study was financed by the Dutch Cancer Society. 


\section{Chapter 4}

\section{Patients}

Institutional review board approval and informed consent were obtained. During the study period of June 2004 to July 2006 a total of eighty-seven consecutive patients were diagnosed with biopsy proven primary rectal cancer at our centre. All rectal tumors were defined by an inferior tumor margin $<15 \mathrm{~cm}$ from the anal verge as described by endoscopy. Fourteen patients with non-resectable disease or contraindications for MRI were excluded from the study. Forty-five patients with locally advanced tumors (involved CRM or $\mathrm{N}_{2}$ status) underwent neoadjuvant chemoradiation, and were excluded because after chemoradiation histology cannot be considered representative of the primary nodal status. Twenty-eight patients were eligible for this prospective study (18 males and 10 females, mean age 68 , range 54-89 years). These patients underwent USPIO MRI before their standard treatment. The standard treatment in our country for nonlocally advanced rectal cancer is a short course of radiotherapy (5x5 Gy) immediately followed by Total Mesorectal Excision Surgery ${ }^{8}$. The short course of radiotherapy is known not to downstage the nodal status in rectal cancer patients ${ }^{9}$.

\section{Ultrasmall Superparamagnetic Iron Oxide}

The USPIO MR contrast agent, Sinerem (Guerbet Laboratories, Roissy, France), consists of low molecular weight iron oxide coated with dextran. Sinerem is supplied as a powder in a glass vial containing $210 \mathrm{mg}$ and must be reconstituted by using $10 \mathrm{~mL}$ of normal saline. A dose of $0.13 \mathrm{~mL}$ per kilogram of body weight (2.6 mg Fe/ $/ \mathrm{kg}$ ) of the reconstituted solution was diluted in $100 \mathrm{~mL}$ of normal saline. This was administered in the preparation room of the MR unit. The contrast agent was given intravenously within a period of approximately 45 minutes by slow drip infusion with a microfilter. Administration was closely monitored for any adverse effects and completed 24-36 hours before post contrast MR imaging was planned. No precontrast MRI was performed. No adverse events relating to the USPIO infusion were observed in this study.

In the literature two main mechanisms are described for transportation of the nanoparticles to the lymph nodes. The major pathway is direct transcapillary passage of the nanoparticles from blood vessels into the medullary sinuses of the lymph node. The other pathway is nonselective endothelial transcytosis across 
permeable capillaries into the interstitium, from where nanoparticles are transported through the lymphatic system and accumulate in macrophages within the lymph node ${ }^{10,11}$. The nanoparticles cause a decrease in signal intensity within the node due to susceptibility artefacts on $3 D T 2 *$-weighted images. Variation of signal intensity within a node can be explained on the basis of the concentration of macrophages (nanoparticles) in a particular region in the node. A region with a normal concentration of macrophages (nanoparticles) will produce an area with signal decrease. This means that enlarged inflamed nodes will also show a significant fall in signal intensity. The involved part of the lymph node will show no signal decrease due to the replacement of macrophages by tumor cells creating a region of increased signal intensity within the node (white region).

\section{MR Imaging}

MR imaging was performed on a 1.5 T system Gyroscan, Powertrak 6000, NT release 6.2.1, 23.0 mT.m, rise time $0.2 \mathrm{~ms}$, slew-rate $105 \mathrm{~T} 1 / \mathrm{ms}$ (Philips Medical Systems, Best, Netherlands) using a pelvic phased array coil with the patient in feet-first, supine position. Sequences used are sagittal and axial 2D T2 weighted TSE. 3DT2*-weighted axial images were obtained with 23/18.41 (TR/TE), 20 degree flip angle, a $512 \times 512$ matrix, and 1.25-mm slice thickness. 3D T1-weighted axial images were obtained with shortest 10/4.60 (TR/TE), 15 degree flip angle, a $384 \times 512$ matrix, and $1 \mathrm{~mm}$ slice thickness to locate lymph nodes and to differentiate them from blood vessels. All axial images were angled perpendicular to the rectal tumor. Patients did not receive bowel or other preparation. The total scan time was 32-40 minutes.

\section{Image Evaluation}

Two observers (1) R.G.H. and (2) M.J.L. (with approximately 3000 and 300 MR pelvic reading experience respectively) prospectively and blinded to each other's results and histological results evaluated each node on axial 3DT2*-weighted images for the following items: border (sharp, indistinct or disrupted), short and long axis diameter. Furthermore the percentage of white region within the node was estimated and categorised as: $<30 \%, 30-50 \%$ or $>50 \%$ on $3 \mathrm{DT} 2 *$-weighted images (see figure 4.1). 


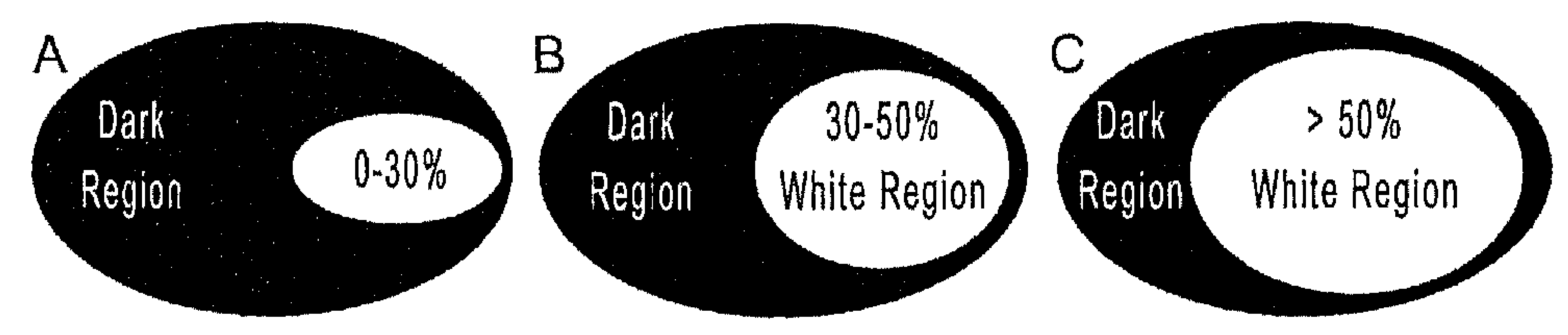

Figure 4.1 A schematic diagram of a lymph node on USPIO enhanced $T_{2} *$-weighted images with (A) white region of $<30 \%$, (B) white region $30-50 \%$ and $(C)$ white region $>50 \%$

Beside this visual assessment of the percentage of white region within the node, the proportion of white region within the node was also quantitatively measured. The surface area of white region within the node and the surface area of total node were determined on an axial $3 D T_{2} *$-weighted image, where the node was the largest, using the workstation's measurement tools. By dividing the surface area of white region by the surface area of the total node, a quantitatively measured ratio of white region was calculated for both observers. This is further referred to as Ratio $_{\mathrm{A}}\left(\frac{\text { Measured surface area of white region }}{\text { Measured surface area of total node }}\right)$. Ratio $_{\mathrm{A}}$ was not categorised.

By placing regions of interests ( $\mathrm{ROI}$ 's) completely over the white and dark region within the node the signal intensity ( $\mathrm{SI}$ ) were measured. ROI's of the same size were used to measure the signal intensity of the total node and that of the gluteus muscle. These signal intensities were used to calculate the following two ratio's for both observers: $S \mathrm{I}_{\text {white }} / S \mathrm{I}_{\text {dark }}\left(\frac{\text { SI of white region }}{S I \text { of dark region }}\right)$ and $S \mathrm{I}_{\mathrm{TN}} / S \mathrm{I}_{\text {muscle }}$ $\left(\frac{\text { SI of total node }}{\text { SI of muscle }}\right)$.

The observer with the highest level of experience, observer 1, also prospectively recorded the localization of each visible lymph node depicted by $3 D T_{2}{ }^{*}$-weighted MR images on an anatomical map (see figure 4.2). This anatomical map was used as a template to compare with the nodes depicted by the other observers (see below) and the nodes found at histopathological evaluation. 
A third observer was introduced to evaluate the most accurate and practical predictive criterion for nodal status of observer $1 \& 2$. Observer 3 (S.M.E.) had similar reading experience as observer 2 (approximately 300 pelvic MR readings).
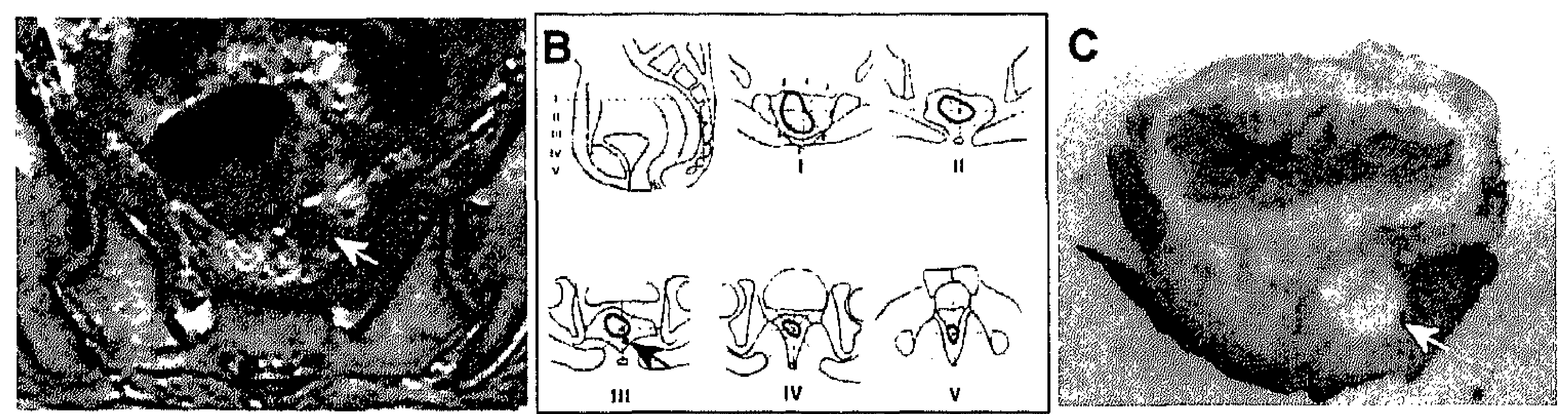

Figure 4.2 The MR radiologist predicted each visible lymph node on MR images (A). Each visible node was recorded on an anatomical 'roadmap'. (B). Each node was harvested from the histological specimen. The anatomical map was used as a template to match the nodes found at the histological evaluation $(C)$ with the nodes depicted on MRI

\section{Histopathological Evaluation}

The standard surgical procedure was a TME, as described by Heald ${ }^{12}$. The rectum with the complete surrounding mesorectal fat and mesorectal lymph nodes was removed by sharp dissection along the mesorectal fascia. Histological examination was standardized: the specimen was fixed in formalin for $24-48 \mathrm{~h}$ and the circumferential resection plane inked. The specimen was then sectioned every $5 \mathrm{~mm}$ transversely and thus also perpendicular to the mesorectum. A careful search for lymph nodes was made in each histological tissue slice by a dedicated pathologist. Each lymph node found was matched to the corresponding node visible on the MR images using the anatomical map as a template. This nodal harvesting procedure was performed by a pathologist (A.P.B., with 17 years of experience) together and side by side with observer 1 to ensure an accurate lesion-by-lesion analysis. Accurate nodal matching was only possible due to the detailed knowledge of the MR images using specific characteristics like the size of the lymph node and the position of the lymph node in relation to the rectal wall, small vessels, mesorectal fascia, tumor and other lymph nodes. In cases in which nodal matching was not possible it was recorded as such. Each harvested lymph node was placed in marked individual trays, and processed according to standard methods and stained with haematoxylin-eosin. Then the same pathologist, blinded to the USPIO MRI predictions, reported the 


\section{Chapter 4}

status of each lymph node: benign or malignant. In this manner accurate lesionby-lesion analysis for mesorectal lymph nodes could be performed.

\section{Statistical analysis}

Based on the results of a pilot study at our center we expect a sensitivity of $99 \%$. 30 positive lymph nodes are needed to reject with a power of $75 \%$ that the lower limit of the $95 \%$ confidence interval of sensitivity of the USPIO MRI is $90 \%$ or lower. The number of patients needed to include 30 positive lymph nodes can be calculated as follows: $10 \%$ of the nodes found will be malignant and an average of 12 nodes in each patient will be found at histopathological examination; this means that we need 300 nodes at histopathological examination and thus 25 patients.

To determine the interobserver agreement, weighted kappas $^{13,14}$ were calculated for the ordinal variables and Intra Class Correlations (ICC) for interval scaled variables ${ }^{15}$, all with their $95 \%$-confidence intervals. A kappa value ( $\mathrm{K}$ value) of less than 0.00 indicates poor agreement; $0.00-0.20$ slight agreement; $0.21-0.40$ fair agreement; $0.41-0.60$ moderate agreement; $0.61-0.80$ substantial agreement and $0.81-1.00$ almost perfect agreement ${ }^{16}$. The ICC ranges from 0 to 1 , with a value of 0 indicating a completely unreliable measurement, and a value of 1 indicating a $100 \%$ reproducible measurement.

Diagnostic performance of the different evaluation methods were analysed with logistic regression model and generalized estimating equations (GEE) were used to estimate marginal effects. With the results receiver operating characteristic (ROC) curves were constructed and area under the curves (AUC's) \& 95\%confidence levels were calculated using STATA software (Stata Statistical Software: Release 9. College Station, TX: StataCorp LP).

\section{RESULTS}

\section{Histology}

In 28 patients MR imaging found 362 lymph nodes in the mesorectum. After surgical resection 333 lymph nodes were found at histology; 27 malignant and 306 benign lymph nodes. Of the $333(27+306)$ nodes there were 236 nodes at histology, that could exactly be matched with nodes found on MRI, while 97 
nodes found at histology could not be exactly matched with MRI. These 97 nodes, all benign, were excluded from the analysis. This leaves us with 236 lymph nodes for the lesion-by-lesion analysis (see figure 4.3). Only 176 node were eligible for SI measurements, because 60 nodes were too small to accurately measure the signal intensity of the node. The mean short- and long axis diameter of the total lymph node were $3.6 \mathrm{~mm}$ (range $2-9 \mathrm{~mm}$ ) and $4.7 \mathrm{~mm}$ (range 2-13 mm), respectively.

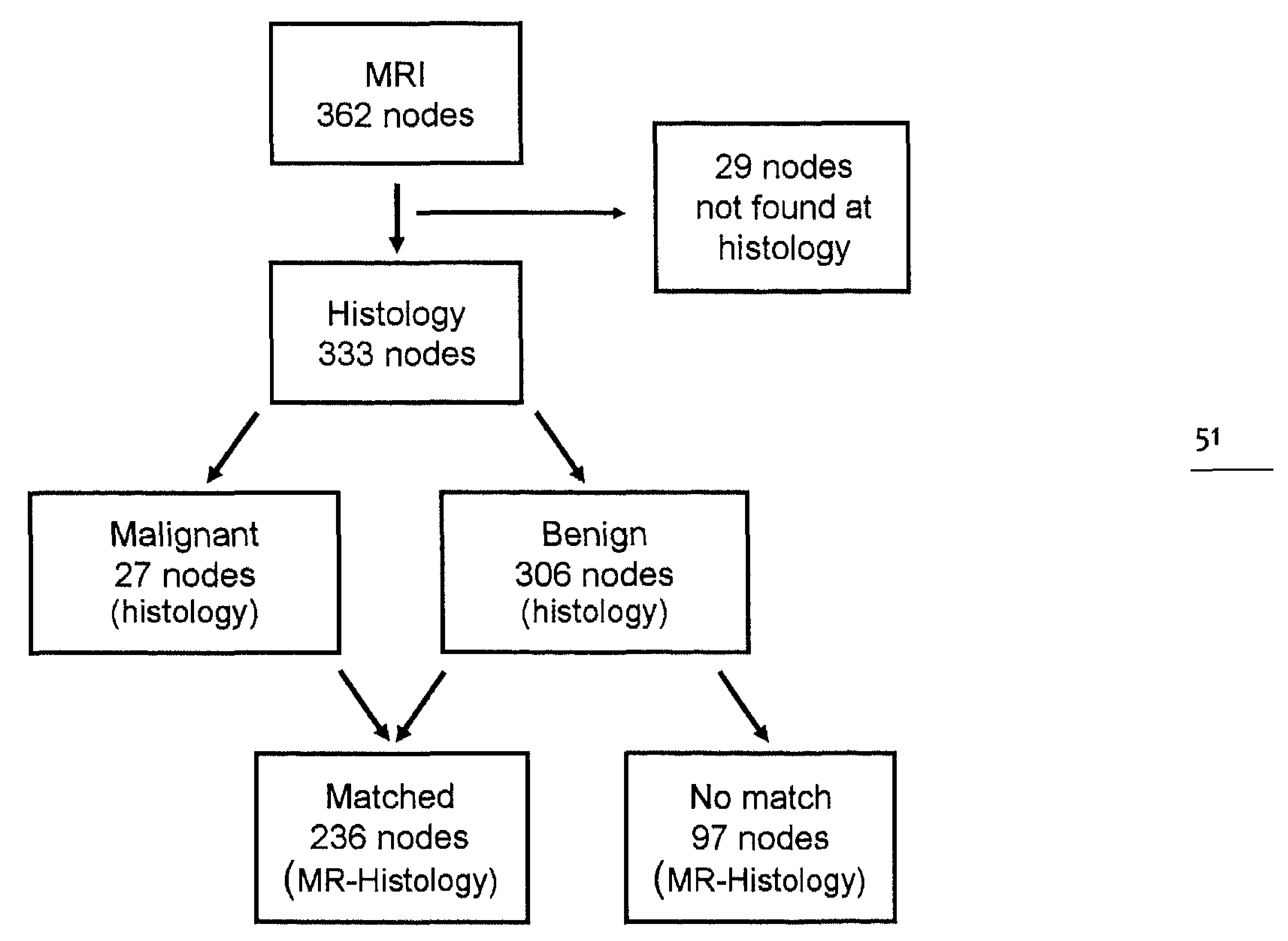

Figure 4.3 A flowchart of the lymph nodes found on MR imaging and at histology

\section{Diagnostic Performance}

The AUC of border, short axis, long axis, $S I_{\mathrm{TN}} /\left.\mathrm{S}\right|_{\text {muscle }}$ and $\left.S\right|_{\text {white }} /\left.S\right|_{\text {dark }}$ for observer 1 were: $0.65,0.75,0.79,0.85,0.75$ and for observer $2: 0.58,0.75,0.79,0.89$ and 0.79 , respectively (see table 4.1 and figure 4.4 ). 


\section{Chapter 4}

The highest AUC for the prediction of the nodal status were found for the estimated percentage and measured Ratio ${ }_{A}$ of white region within the node $(0.96$ and 0.99 respectively for observer 1 and 0.95 and 0.97 respectively for observer 2). When the white region within the node is estimated as more than $30 \%$, the sensitivity and specificity for detection of malignant nodes is $93 \%$ and $96 \%$ respectively (see figure 4.5 and 4.6 ).

\begin{tabular}{lccccc}
\hline & \multicolumn{4}{c}{ Observer 1 } & \multicolumn{2}{c}{ Observer 2 } \\
& AUC & $95 \% \mathrm{CL}$ & AUC & $95 \% \mathrm{CL}$ & $\mathrm{N}$ \\
\hline Short $\varnothing \mathrm{LN}$ & 0.75 & $0.61-0.89$ & 0.75 & $0.64-0.87$ & 236 \\
Long $\varnothing \mathrm{LN}$ & 0.79 & $0.67-0.91$ & 0.79 & $0.69-0.90$ & 236 \\
Border & 0.65 & $0.60-0.79$ & 0.58 & $0.48-0.68$ & 236 \\
Estimated \% white region & 0.96 & $0.90-1.0$ & 0.95 & $0.90-1.0$ & 236 \\
Ratio $_{\mathrm{A}}$ & 0.99 & $0.98-1.0$ & 0.97 & $0.91-1.0$ & 236 \\
$\mathrm{SI}_{\mathrm{TN}} / \mathrm{SI} I_{\text {muscle }}$ & 0.85 & $0.75-0.94$ & 0.89 & $0.80-0.97$ & 176 \\
$\mathrm{SI}_{\text {white }} / \mathrm{SI}_{\text {dark }}$ & 0.75 & $0.60-0.90$ & 0.79 & $0.66-0.91$ & 176 \\
\hline Table 4.1 Area under the curve (AUC) and 95\%-Confidence Intervals for observer 1 and 2 for all criteria used to \\
distinguish between malignant and benign nodes
\end{tabular}

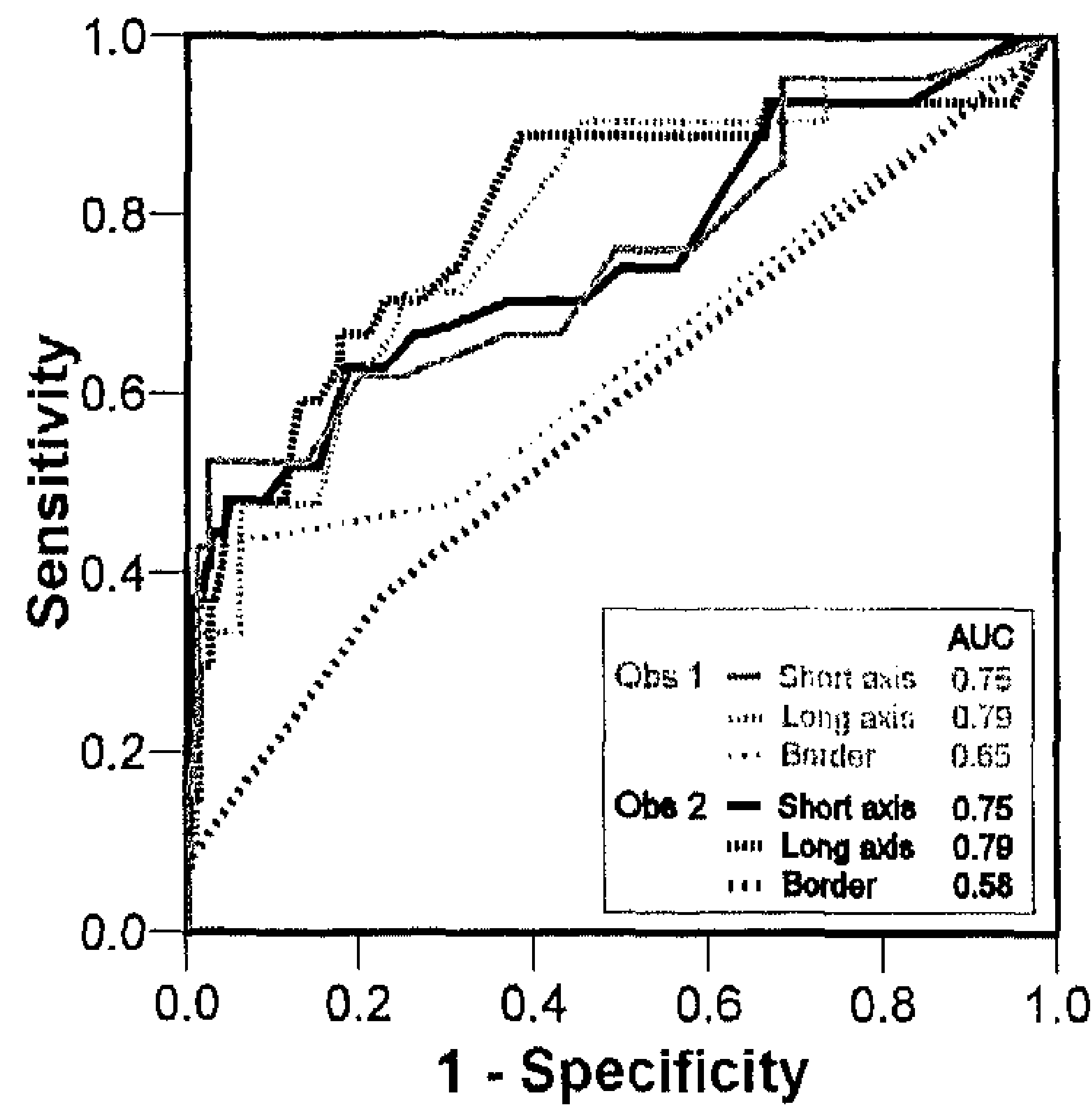

Figure 4.4 Receiver operating characteristic (ROC) curves and areas under the curve (AUC) for observer 1 and 2, for the detection of malignant lymph nodes using the short \& long axis diameter and border of the lymph node

Of the two criteria that were the most accurate in predicting the nodal status with USPIO MRI, the estimated percentage of white region within the node was 
the most practical to use in the clinical practice. Therefore this criterion was chosen to be evaluated by observer 3. The AUC (95\%-confidence level) of observer 3 for this criterion was 0.96 (0.91-1.0).

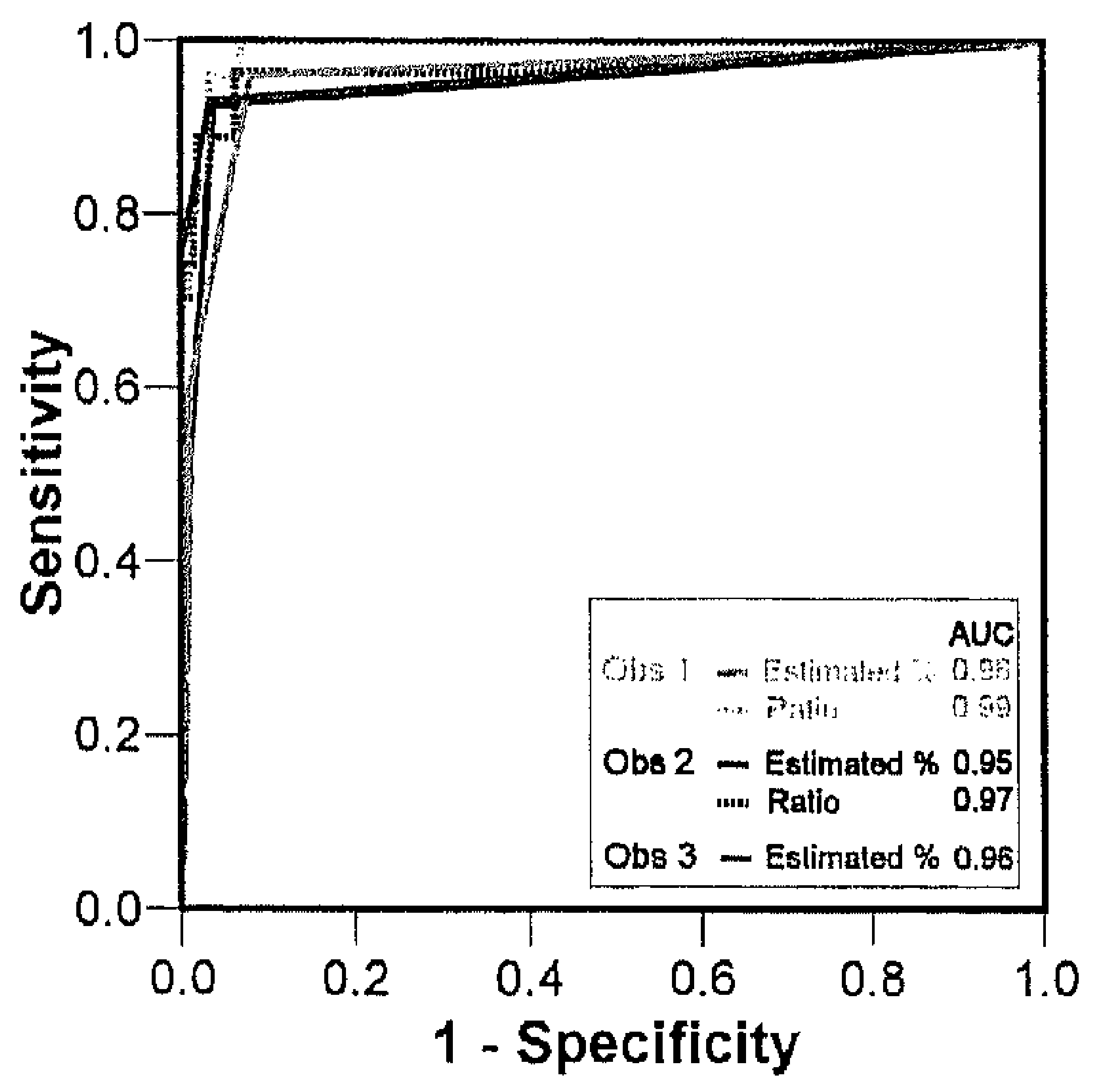

Figure 4.5 Receiver operating characteristic (ROC) curves and areas under the curve (AUC) (observer 1, 2 \& 3) for the detection of malignant lymph nodes using the estimated \% of white region within the node and the measured Ratio (observer 1 \& 2)

ICC

The Intra Class Correlation for observer $1 \& 2$ for the short axis, long axis diameter, the Ratio ${ }_{A}$, the $S I_{T N} / S I_{\text {muscle }}$ and $S I_{\text {white }} / S I_{\text {dark }}$ were $0.91,0.96,0.91,0.72$ and 0.92 (see table 4.2). Generally the interobserver agreement was good to excellent. There was more agreement for the Ratio ${ }_{A}$, short and long axis diameter than for the $S I_{T N} / S I_{\text {muscle }}$.

Interobserver Agreement

The interobserver agreement ( $K$ value) for the estimated white region between observer $I$ and 2 was good (0.77) while that for the border was only fair $(0.28)$. The $K$ value analysis of the estimated white region for observer $1 \& 3$ and observer $2 \& 3$ were good $(0.79)$ and excellent (0.84), respectively. 


\section{Chapter 4}
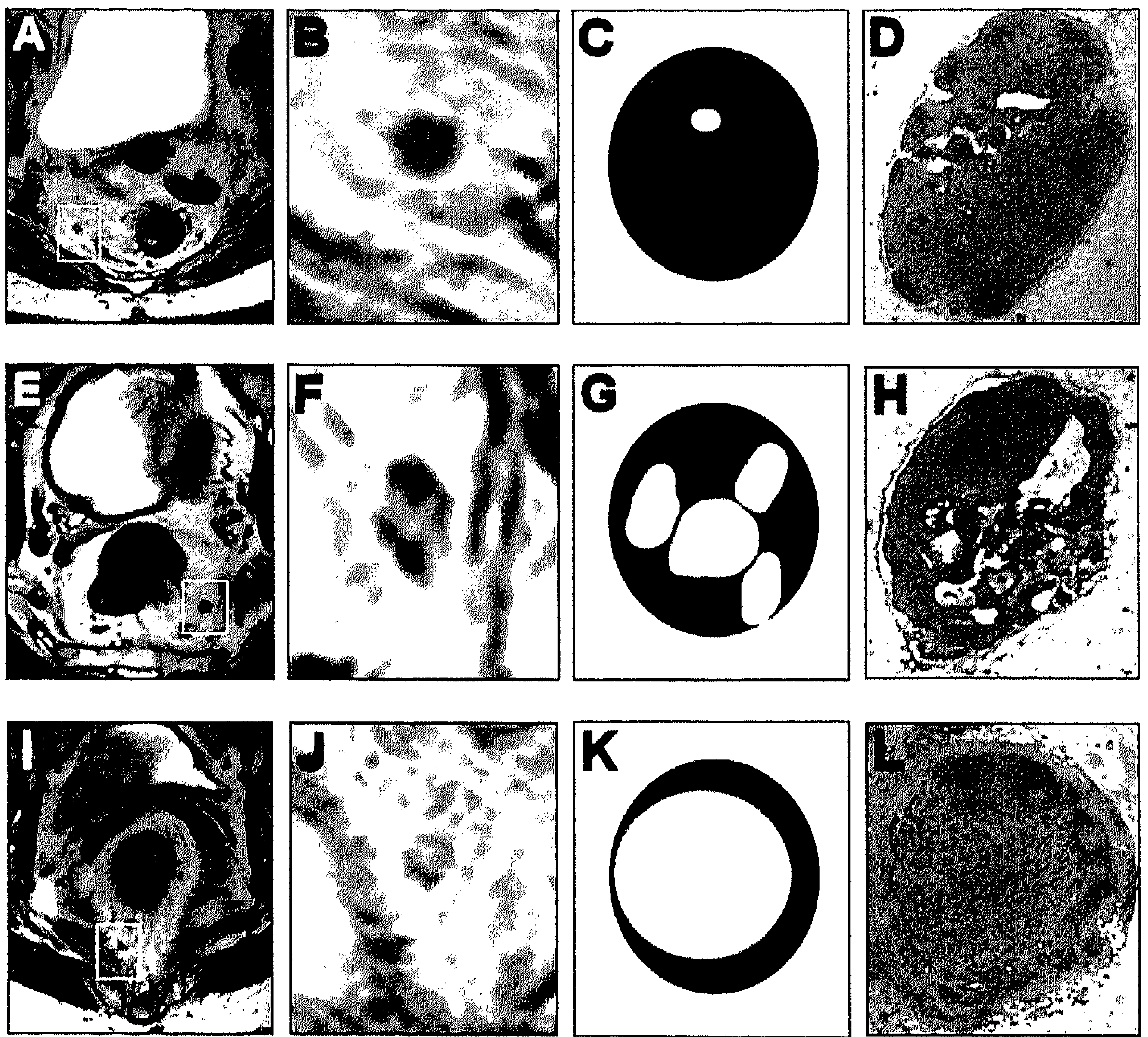

Figure 4.6 $(A, B, C, D) A$ benign mesorectal node, diagnosed on USPIO enhanced MRI: (A) on T2-weighted FSE image smaller than $5 \mathrm{~mm}$ and difficult to differentiate between malignant and benign, $(B)$ on USPIO enhanced $T_{2} *$ image causing a black blooming artefact with (C) less than $30 \%$ white region within the node indicating a benign node, $(D)$ at histology confirmed to be a benign node

Figure 4.6 $(E, F, G, H)$ A small malignant mesorectal node, detected with USPIO MRI; (E) on T2-weighted FSE image smaller than $5 \mathrm{~mm}$ and difficult to differentiate between malignant and benign, (F) on USPIO enhanced $T_{2} *$ image showing incomplete blackening of the node, (G) with an estimated 30-50\% white region within the node, suggesting a malignant node, $(H)$ at histology confirmed to be a partially involved metastatic node

Figure $4.6(I, J, K, L)$ An ultrasmall malignant mesorectal node, detected with USPIO MRI: (I) on T2-weighted FSE image smaller than $3 \mathrm{~mm}$ and difficult to differentiate between malignant and benign, $(J)$ on USPIO enhanced $T_{2}$ * image remaining white indicating no iron uptake, $(K)$ with more than $50 \%$ white area within the node, suggesting a malignant node, (L) at histology confirmed a massively involved tumoral node 
Predictive Criteria for Nodal Staging

\begin{tabular}{lcc}
\hline & Intra Class Correlation & $95 \% \mathrm{CL}$ \\
\hline Short $\varnothing \mathrm{LN}$ & 0.91 & $0.87-0.94$ \\
Long $\varnothing \mathrm{LN}$ & 0.96 & $0.97-0.99$ \\
Ratio $_{\mathrm{A}}$ & 0.91 & $0.87-0.94$ \\
$\mathrm{SI}_{\mathrm{TN}} / \mathrm{SI} \mathrm{I}_{\text {muscle }}$ & 0.72 & $0.57-0.82$ \\
$\mathrm{SI}_{\text {white }} /\left.\mathrm{SI}\right|_{\text {dark }}$ & 0.92 & $0.86-0.96$ \\
\hline Table 4.2 Intra Class Correlations (ICC) and 95\%-confidence intervals for all for interval scaled criteria for observer 1 \\
and 2
\end{tabular}

\section{DISCUSSION}

Our results show that for observer 1 and 2 both the estimated as well as the measured ratio of the white region within the node were very accurate predictors for malignant nodes in rectal cancer. The results of observer 3 confirmed that the estimated percentage of the white region within the node could serve as a reliable and practical criterion for the prediction of malignant nodes on USPIO MRI.

Size criteria, such as long and short axis of the node, and border morphology were only moderately accurate for the distinction between malignant and benign lymph nodes.

An estimated area of white region within the node larger than $30 \%$, was highly predictive for an involved node with a sensitivity of $93 \%$ and a specificity of $96 \%$. The measured Ratio ${ }_{A}$ was also an accurate predictor for malignant lymph nodes. The larger the area of white region, the more likely the node is malignant. The white region in the lymph node is caused by no or very little uptake of USPIO in that malignant part of the lymph node. Benign conditions like focal nodal fibrosis, granulamatous disease or a fatty hilum can also be depicted as a white region due to the lack of macrophages, thus mimicking malignant nodes. These white regions however are usually $30 \%$ or less of the total node area. Another way to differentiate between a white fatty hilum and a white tumoral region is to compare the $3{ }_{3} D T_{2} *$-weighted images with the ${ }_{3} D T_{1}$-weighted images where a fatty hilum is depicted as a white region within the node, while tumor is not. In the same manner the dark region can hide small micrometastases. The clinical implication of micrometastases however is debatable. Although there is a small 
overlap in MR features of benign and malignant nodes, a general rule of thumb is that when the white region is less than $30 \%$ the nodes are most often benign.

To our knowledge no literature is published concerning the estimated or measured ratio of the white region within the node. A range of patterns of contrast enhancement have been described to discriminate between malignant and non-malignant lymph nodes using USPIO MRI ${ }^{17}$. The use of patterns to predict nodes has a major drawback. The radiologist's mind must choose from a variety patterns and assess which of the patterns would fit best to a malignant and which to a benign node. This involves a certain level of subjectivity and constitutes a potential source of erroneous interpretation. Our study shows that a simple estimation or measurement of the white region within the node is reliable for the identification of malignant nodes. The interobserver agreement between an experienced reader and less experienced readers (observer 1 vs. observer $z$ or 3) for the estimated white region were good, indicating a high consistency in interpreting the images independent of the experience of the readers. The $K$ value of 0.84 for observer 2 vs. 3 , even shows that two less experienced readers can have an excellent agreement among each other. These findings suggest that USPIO MRI could become a promising tool for nodal malignancy prediction not only for experienced but also for less experienced readers.

The $S I_{T N} /\left.S\right|_{\text {muscle }}$ measurements show that the higher the signal intensity of the total node is, the higher the risk of a malignant node. These findings confirm earlier reports of signal intensities in USPIO MRI. Harisinghani et al. ${ }^{18}$ demonstrated that a distinction between malignant and benign nodes could be made on the basis of signal intensity of the total node. In our study the SI ratio measurements between the white and dark region within the node $\left(S I_{\text {white }} / S I_{\text {dark }}\right)$ were disappointing. The moderate AUC can be explained by the difficulties of measuring a white and a dark region within a very tiny node. The white and dark region within the node are often too small or too irregular to accurately position a ROI. In this study $26 \%$ of all nodes could not be measured, obviously making this criterion less valuable in clinical practice.

Size criteria were insufficient to consistently distinguish between malignant and benign lymph nodes in patients with rectal cancer. The disappointing results concerning the size criteria are due to the high rate of relatively small involved lymph nodes in rectal cancer. Brown et. al ${ }^{19}$ showed that even mesorectal lymph 
smaller than $5-\mathrm{mm}$ still had a prevalence of positive lymph nodes of $15 \%$. As early as 1989 Dworak et al. ${ }^{20}$ showed that $32 \%$ of the patients with involved nodes had only small ( $5 \mathrm{~mm}$ or less) involved nodes. This partly explains why EUS, CT and nonenhanced MR studies on nodal prediction in rectal cancer patients using size criteria have shown disappointing results ${ }^{6}$. Additional MR criteria have therefore been studied, such as border and signal intensity. Kim et al. ${ }^{21}$ and Brown et al. ${ }^{19}$ found that the border and signal intensity characteristics of lymph nodes on $T 2 W$ FSE images were useful for identification of malignant nodes in rectal cancer. The aspect of the border and the homogeneity of the signal however are generally easier to evaluate in the 'larger' nodes $(>5 \mathrm{~mm}$ ). In our study however we mainly included patients with non-locally advanced tumors with predominantly smaller nodes. In our experience USPIO MRI and its characteristics are especially of additional benefit in the evaluation of these small nodes $(<5 \mathrm{~mm})$, where border and signal intensity criteria tend to be less helpful.

A limitation of our study is the relative limited number of positive nodes. This is due to the study's patient selection of 'non-locally advanced' rectal cancer stratified for a short preoperative radiation course in order to be able to perform a lesion-by-lesion analysis. Another issue is that no preoperative radiation would probably have been more convincing for exact lesion-by-lesion correlation. However, nowadays that standard preoperative radiotherapy has definitely shown benefit for the reduction of the local recurrence rate in rectal cancer patients, treating patients with non-superficial rectal cancer without preoperative radiation would be unethical. Furthermore literature shows that no downstaging occurs after short-term of preoperative radiation ${ }^{9}$.

In conclusion the estimated percentage of white region within the node and the measured Ratio ${ }_{A}$ are the most accurate predictive criteria for USPIO MRI. In the busy daily practice of a radiologist there is little room for time-consuming measurements of numerous nodes. Therefore the estimated percentage of white region within the node would be most practical to use. Our study indicates that this was an accurate criterion not only among experienced and less experienced readers, but also for two less experienced readers. 


\section{Chapter 4}

\section{REFERENCES}

1.

$1996 ; 83(3): 293-304$.

2. Pahlman L, Glimelius B. Pre- or postoperative radiotherapy in rectal and rectosigmoid carcinoma. Report from a randomized multicenter trial. Ann Surg 1990;211(2): 187-195.

3. Saver R, Becker H, Hohenberger W, Rodel C, Wittekind C, Fietkau R, Martus P, Tschmelitsch J, Hager $E$, Hess CF, Karstens JH, Liersch T, Schmidberger $H$, Raab R. Preoperative versus postoperative chemoradiotherapy for rectal cancer. N Engl J Med 2004;351(17): 1731-1740.

4. Moran MR, James EC, Rothenberger DA, Goldberg SM. Prognostic value of positive lymph nodes in rectal cancer. Dis Colon Rectum 1992;35(6): 579-581.

5. Park YJ, Park KJ, Park JG, Lee KU, Choe KJ, Kim JP. Prognostic factors in 2230 Korean colorectal cancer patients: analysis of consecutively operated cases. World J Surg 1.999;23(7):721-726.

6. Lahaye MJ, Engelen SM, Nelemans PJ, Beets GL, van de Velde CJ, van Engelshoven JM, Beets-Tan RG. Imaging for predicting the risk factors--the circumferential resection margin and nodal disease-of local recurrence in rectal cancer: a meta-analysis. Semin Ultrasound CTMR 2005;26(4): 259-268.

7. Will O, Purkayastha 5, Chan C, Athanasiou T, Darzi AW, Gedroyc W, Tekkis PP. Diagnostic precision of nanoparticle-enhanced MRI for lymph-node metastases: a meta-analysis. Lancet Oncol 2006;7(1): 52-60.

8. Kapiteijn E, Marijnen CA, Nagtegaal ID, Putter H, Steup WH, Wiggers T, Rutten HJ, Pahlman L, Glimelius $B$, van Krieken $\mathrm{JH}$, Leer JW, van de Velde CJ. Preoperative radiotherapy combined with total mesorectal excision for resectable rectal cancer. N Engl J Med 2001;345(9): 638-646.

58 9. Marijnen CA, Nagtegaal ID, Klein Kranenbarg E, Hermans J, van de Velde CJ, Leer JW, van Krieken JH. No downstaging after short-term preoperative radiotherapy in rectal cancer patients. $J$ Clin Oncol 2001;19(7): 1976-1984.

10. Weissleder $R$, Elizondo $G$, Wittenberg J, Rabito CA, Bengele $H H$, Josephson L. Ultrasmall superparamagnetic iron oxide: characterization of a new class of contrast agents for MR imaging. Radiology $1990 ; 175(2): 489-493$.

11. Frija G, Clement $O$, Le Guen O, Cuenod CA, Siauve $N$, Benderbous S. Experimental investigation of the delivery pathway of ultrasmall superparamagnetic iron oxide to lymph nodes. Acad Radiol 1996;3 Suppl 2: S299-300.

12. Heald RJ, A new approach to rectal cancer. Br J Hosp Med 1979;22(3): 277-281.

13. Cohen J. A coefficient of agreement for nominal scales. Educational and Psychological Measurement 1960;20: 37-46.

14. Cohen J. Weighted Kappa: nominal scale agreement with provision for scaled disagreement or partial credit. Psychol Bull 1968;70: 213-230.

15. Shrout PE, Fleiss J.L. Intraclass Correlations: Uses in Assessing Rater Reliability. 1974;86(2): $420-428$.

16. Landis JR, Koch GG. The measurement of observer agreement for categorical data. Biometrics 1977;33(1): 159-174.

17. Koh D-M, Brown G, Temple L, Raja A, Toomey P, Bett N, Norman AR, Husband JE. Rectal Cancer: Mesorectal Lymph Nodes at MR Imaging with USPIO versus Histopathologic Findings--Initial Observations. Radiology 2004;231(1): 91-99.

18. Harisinghani MG, Saini S, Slater GJ, Schnall MD, Rifkin MD. MR imaging of pelvic lymph nodes in primary pelvic carcinoma with ultrasmall superparamagnetic iron oxide (Combidex): preliminary observations. J Magn Reson Imaging 1997;7(1): 161-163. 
Predictive Criteria for Nodal Staging

19. Brown G, Richards CJ, Bourne MW, Newcombe RG, Radcliffe AG, Dallimore NS, Williams GT. Morphologic Predictors of Lymph Node Status in Rectal Cancer with Use of High-Spatial-Resolution MR Imaging with Histopathologic Comparison. Radiology 2003;227(2): 371-377.

20. Dworak O. Number and size of lymph nodes and node metastases in rectal carcinomas. Surg Endosc $1989 ; 3(2): 96-99$

21. Kim JH, Beets GL, Kim MJ, Kessels AG, Beets-Tan RG. High-resolution MR imaging for nodal staging in rectal cancer: are there any criteria in addition to the size? Eur J Radiol 2004;52(1): 78-83. 


\section{MR prediction of the risk factors - CRM, T-stage and nodal status - in primary rectal cancer: a multicenter study}

M.J. Lahaye, S.M.E. Engelen, G.L. Beets, A.G.H. Kessels,

P.J.M. Post, J.P.M. Dohmen, G.R.J. Opdenakker, C.J.H. van de Velde, J.M.A. van Engelshoven, R.G.H. Beets-Tan 


\section{Chapter 5}

\section{ABSTRACT}

Aim To evaluate the accuracy of USPIO MRI to predict the distance to the mesorectal fascia, $\mathrm{T}$ - and $\mathrm{N}$-stage in primary staging of rectal cancer in patients not receiving chemoradiation in the setting of a general hospital and the setting of an expert center.

Methods Between February 2003 and January 2008 a multicenter prospective rectal cancer project included 296 patients with biopsy proven primary rectal cancer. Patients underwent MRI 24hrs. after i.v. administration of USPIO. Sequences used were axial 2D T2W FSE, 3D T1W GRE \& $3 D T_{2} *$. The general radiologist and an experienced $M R$ radiologist prospectively predicted the circumferential resection margin (CRM), T-stage and the nodal status first on $T_{2}$ TSE images (USPIO-), then on the combined T2 FSE and $3 \mathrm{BTT}_{2} *$ weighted images (USPIO+), blinded for each other's results. The expert prospectively double read each MR of the regional study patients. Mainly on the basis of these risk factors for local recurrence the treatment strategy was decided: surgery only, surgery after $5 \times 5$ Gy RT, or surgery after a long course of chemoradiation. In 59 patients surgery was performed without any neoadjuvant treatment and in 91 patients after $5 \times 5$ Gy RT. These 150 were included in the present study, as only in these patients the histology can serve as the reference standard.

Results The prediction of CRM had an NPV of $100 \%$. The expert radiologist had a PPV for prediction of pTo-2 of $95 \%$, for the general radiologists (as a group) $87 \%$ resp. The AUC and NPV for prediction of malignant nodes on T2 FSE images for the expert radiologist was 0.84 and $92 \%$, for the general radiologists (as a group) 0.81 and $83 \%$ resp. The AUC and NPV for prediction of malignant nodes on USPIO enhanced images for the expert radiologist was 0.92 and $97 \%$, for the general radiologists (as a group) 0.87 and $95 \%$ resp. If the latter two risk factors (T- \& N-stage) were combined expert and general radiologists could also accurately predict Stage I disease with a PPV of 0.94 and 0.92 respectively.

Conclusion Expert and general radiologists can accurately predict a noninvolved CRM with MR imaging. USPIO MRI can accurately predict tumors limited to the bowel wall with a high PPV and select No patients with a high NPV for the prediction of nodal disease. Stage I disease can reliably be predicted in both referral and general setting. 


\section{INTRODUCTION}

Colorectal cancer is one of the leading causes of death in the Western World. In rectal cancer patients the specific problem is the high rate of local recurrences ${ }^{1-3}$. Nowadays clinicians can choose between a range of therapeutic options: from a local excision for the early tumors to a more invasive treatment such as extensive resections combined with neoadjuvant chemo- and/or radiotherapy for the more locally advanced tumors. Reliable preoperative imaging of the risk factors for local recurrence is essential to choose the most optimal treatment. EUS, CT and MRI are currently used for this. purpose. At least in many European centers the distance of the tumor to the mesorectal fascia, and the corresponding prediction of a close or involved resection margin is considered one of the most important factor that guides the choice between the different neoadjuvant treatment options. In many single center studies MRI has been shown to be very accurate in the assessment of the extent of the primary tumor, and the relation to the mesorectal fascia. A multicenter study by the Mercury Study Group confirmed this ${ }^{4}$. In other settings, such as in many US centers, the clinical T-and N-staging is more used for decisions on neoadjuvant therapy than the anticipated CRM. Patients with stage I disease ( $\mathrm{T}_{1-2 \mathrm{NoMo}}$ ) have repeatedly been shown to have a very low risk for local recurrence and do not benefit from neoadjuvant therapy $y^{5-7}$. Conventional nodal staging with EUS, CT and MRI however is only moderately accurate, with a recent meta-analysis reporting an overall sensitivity of 55 to $67 \%$ and specificity of 74 to $78 \% \%^{8}$. An improved method of nodal imaging would not only be of benefit for the selection of stage I disease, but could also be of help in selecting patients for a local excision, and in the follow up of patients after a local excision of a small rectal cancer. Lymph node specific MR contrast agent could provide a higher accuracy. Will et al. reported encouraging results in a metaanalysis on the MR contrast agent Ultrasmall Superparamagnetic Iron Oxide (USPIO) for predicting tumoral nodes in a wide variety of cancers ${ }^{9}$.

The purpose of the present study to evaluate the accuracy of MRI with USPIO to predict the most commonly used risk factors for local recurrence in rectal cancer: distance to mesorectal fascia, $\mathrm{T}$ and $\mathrm{N}$ stage in primary staging of rectal cancer. $\mathrm{A}$ second purpose was to evaluate the MR performance in the setting of a general hospital as compared to the setting of an expert center. 


\section{Chapter 5}

\section{METHODS}

\section{Patients}

Between February 2003 and January 2008 a multicenter prospective rectal cancer project included 296 patients with biopsy proven primary rectal cancer. The tumor was considered rectal when the inferior margin was not further than $15 \mathrm{~cm}$ from the anal verge on endoscopy. The Institutional review boards from all participating centers approved the project. All patients gave written informed consent. The project was funded by the Dutch Cancer Society. The MRI contrast agent USPIO, not commercially available during the study period, was provided free of charge by Guerbet Laboratories (Roissy, France) as vials of Sinerem . Guerbet Laboratories had no control of inclusion or data.

The main aim of the project was to study the local control after a differentiated treatment with three treatment options: surgery only, surgery after a short course $5 \times 5$ Gy radiotherapy, and surgery after a long course of chemoradiation. The treatment choices were based on a risk assessment for local recurrence that relied mainly on MR imaging. Tumors were classified as low, intermediate, and high risk based on the distance of the tumor to the mesorectal fascia, the nodal status, T-stage and distance of the tumor from the anal margin (see figure 1.1, general introduction).

For the present study that addresses the accuracy of MRI in assessing the risk factors all patients were included in whom the standard of reference of histological examination after surgery was available. Histology was not available in 45 patients who were not operated because of widespread metastatic disease and/or comorbidity (37) or patient refusal (8). 5 patients were excluded because of MRI-related problems like artifacts and refusal. 95 patients who were treated with neoadjuvant chemoradiation were excluded because the histology is no longer representative of the status at initial presentation due to the downsizing and downstaging effect of the neoadjuvant treatment. 1 patient was excluded for the same reason because chemotherapy was administered between the MRI and the rectal resection because of a coexisting malignancy (see figure 5.1).

This results for the present study in a group of 150 patients ( 83 men, 67 women, mean age 69.8 years, range $38-92$ years). In 59 patients surgery was performed without any neoadjuvant treatment and in 91 patients after $5 \times 5$ Gy radiotherapy. 
A short radiotherapy scheme with immediate surgery is known not to downstage the T-stage or nodal status, and histology can be used as the standard of reference ${ }^{10}$.

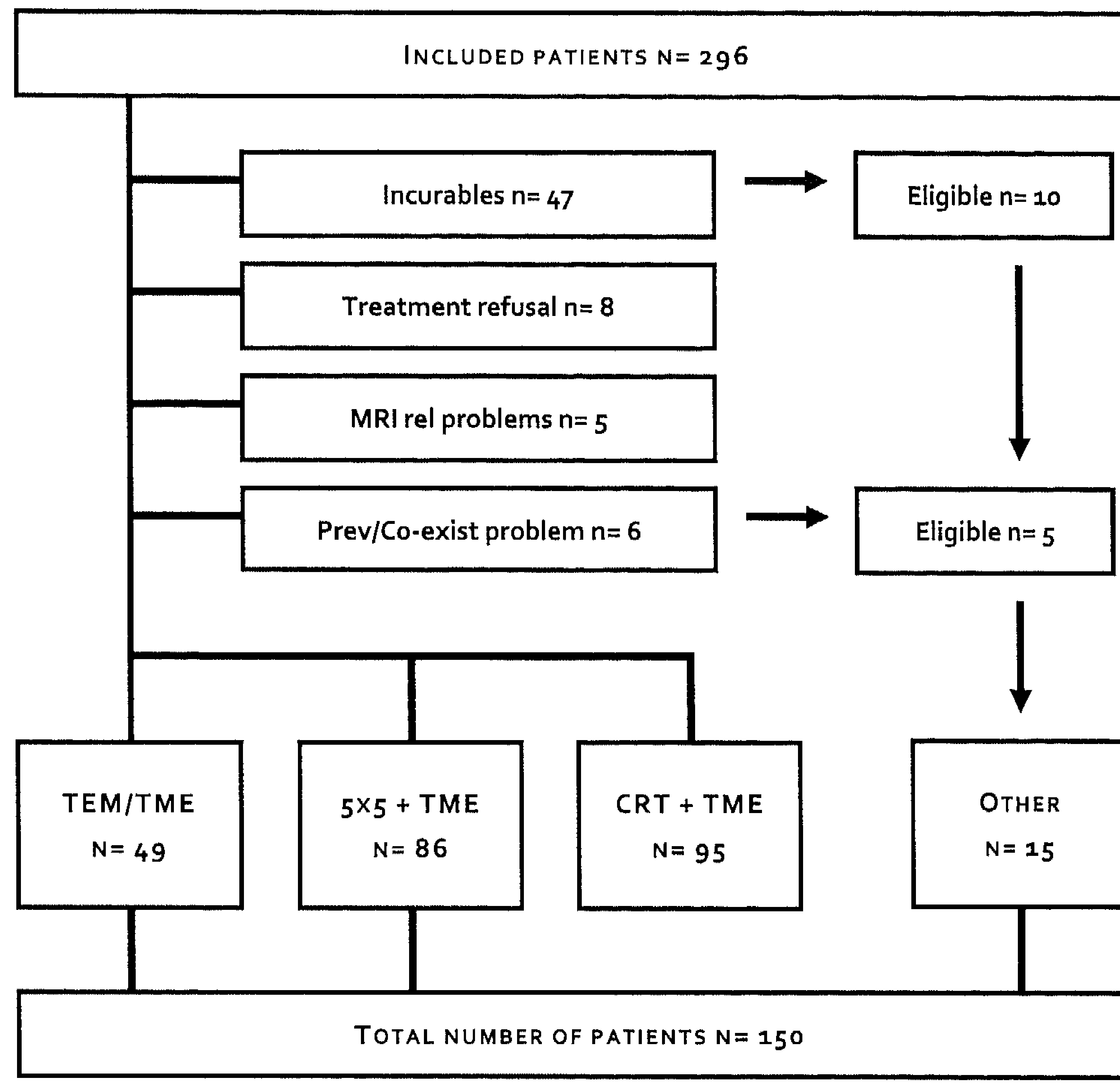

Figure 5.1 Flowchart of patient inclusion

\section{Ultrasmall Superparamagnetic Iron Oxides}

The USPIO MR contrast agent, Sinerem ", consists of nanoparticles iron oxide coated with dextran. Sinerem is supplied as a powder in a glass vial containing $315 \mathrm{mg}$ and must be reconstituted by using $15 \mathrm{~mL}$ of normal saline. A dose of 0.13 $\mathrm{mL}$ per kilogram of body weight $(2.6 \mathrm{mg} \mathrm{Fe} / \mathrm{kg}$ ) of the reconstituted solution was diluted in $100 \mathrm{~mL}$ of normal saline. This was administered intravenously through 
a microfilter within a period of approximately 45 minutes. Administration was performed at the preparation room of the MRI unit or at the day care unit in other centers. Administration was completed $24-36$ hours before MR imaging was planned. The nanoparticles iron oxide accumulate in macrophages within the lymph node ${ }^{11,12}$ and cause a decrease in signal intensity within the node due to susceptibility artefacts on $\mathrm{T}_{2}{ }^{*}$-weighted images. The metastatic part of the lymph node will show no signal decrease and will be depicted as a white region within the lymph node due to the replacement of macrophages by tumor cells. An estimated area of white region within the node larger than $30 \%$ is highly predictive for a malignant node with a sensitivity of $93 \%$ and a specificity of $96 \%$ on a nodal basis ${ }^{13}$.

\section{MR Imaging}

Patients did not receive bowel or any other preparation. All axial images were angled perpendicular to the rectal tumor. The total scan time was 32-45 minutes. Sequences used are sagittal and axial 2D T2 weighted TSE for predicting the Tstage and the nodal status. $\mathrm{T}_{2}$-weighted images were obtained in all patients and were used to interpreter the USPIO uptake patterns within the nodes. $3 \mathrm{D}$ T1weighted images were obtained to locate lymph nodes and to differentiate them from other anatomic structures like small blood vessels. Two regional hospitals had a Siemens Magnetom Impact Expert 1.oT (Siemens AG, Erlangen, Germany) while the university and one regional hospital had a 1.5T MRI (Intera (Philips Medical Systems, Best, Netherlands) and Siemens Symphony $1.5 \mathrm{~T}$ (Siemens AG, Erlangen, Germany), respectively. Overall 130 of 150 patients were scanned at 1.5T MRI and 20 at 1.0T MRI. See table 5.1 for MR sequences and parameters.

\section{Image Evaluation}

All MR images of the general hospitals were first read and interpreted prospectively by the general radiologist of that center (experience: $20-40$ rectal cancer MR readings a year (around 5 years experience)). MR images were also sent to the university center, where the images were prospectively double read by an expert radiologist (R.B.T. with 150 rectal cancer MR readings a year (10 years experience)), blinded for the general radiologist's results. The MR images of patients of the university center were only interpreted by the expert radiologist. 
MR Prediction of CRM, T- and N-stage

To assess the quality of the MRI images all MR evaluations were rated by all readers and scored as 'poor' or 'good'.

\begin{tabular}{|c|c|c|c|c|}
\hline \multirow{2}{*}{$\begin{array}{l}\text { Sequences and parameters } \\
\text { 2D T2W FSE }\end{array}$} & \multicolumn{2}{|c|}{ 1.0T MRI Unit } & \multicolumn{2}{|c|}{1.5 T MRI Unit } \\
\hline & A & B & $C$ & D \\
\hline Repetition time/Echo time (msec) & $5289 / 120$ & $5289 / 120$ & $3427 / 150$ & $3427 / 150$ \\
\hline Slice Thickness (mm) & 5 & 5 & 5 & 5 \\
\hline Flip angle (degrees) & 90 & 90 & 90 & 90 \\
\hline Matrix & $256 \times 256$ & $256 \times 256$ & $256 \times 256$ & $256 \times 256$ \\
\hline $\mathrm{FOV}(\mathrm{mm})$ & 250 & 250 & 200 & 200 \\
\hline No. of slices & 22 & 22 & 22 & 22 \\
\hline \multicolumn{5}{|l|}{ Axial 3D TIW Gradient Echo } \\
\hline Repetition time/Echo time (msec) & $15 / 7$ & $14.30 / 7$ & $10 / 4.60$ & $9.8 / 4.76$ \\
\hline Slice Thickness (mm) & 2 & 2 & 1 & 1 \\
\hline Flip angle (degrees) & 15 & 15 & 15 & 15 \\
\hline Matrix & $256 \times 256$ & $256 \times 256$ & $384 \times 512$ & $384 \times 512$ \\
\hline $\mathrm{FOV}(\mathrm{mm})$ & 300 & 300 & 440 & 440 \\
\hline No. of slices & 102 & 102 & 200 & 208 \\
\hline \multicolumn{5}{|l|}{ Axial $3 D T_{2} * W$} \\
\hline Repetition time/Echo time (msec) & $17 / 7$ & $17 / 7$ & $23 / 18.41$ & $38 / 18$ \\
\hline SliceThickness (mm) & 2 & 2 & 1.25 & 2 \\
\hline Flip angle (degrees) & 20 & 20 & 20 & 8 \\
\hline Matrix & $256 \times 256$ & $256 \times 256$ & $512 \times 512$ & $512 \times 512$ \\
\hline $\mathrm{FOV}(\mathrm{mm})$ & 300 & 300 & 440 & 440 \\
\hline No. of slices & & 106 & 200 & 208 \\
\hline
\end{tabular}

The anticipated CRM was measured on axial $T_{2}$ weighted images as the shortest distance between the tumor and the mesorectal fascia. If this distance was less than $2 \mathrm{~mm}$ it was recorded as an involved margin. In all other cases the anticipated CRM was considered as noninvolved.

The assessment of T-stage was performed on standard axial images (T2 FSEweighted images). Radiologists were asked to predict the tumor stage from $\mathrm{T}_{1}$ to $\mathrm{T}_{4}$. As however the distinction between $\mathrm{T}_{1}$ and $\mathrm{T}_{2}$ is notoriously difficult on $\mathrm{MRI}$, and only the distinction between $\mathrm{T}_{1-2}$ tumors and $\mathrm{T}_{3}-4$ tumors was considered relevant in the decision process, the data were dichotomized along this 
Chapter 5

distinction. A tumor was considered as T1-2 (limited to the bowel wall) when the bowel wall was depicted as an intact line of low signal intensity.

The nodal status on a patient basis was prospectively scored using a confidence level score (definitely node negative $=0$ to definitely node positive $=4$ ). This was first based on the conventional T2-weighted FSE images and thereafter on the combination of T2-weighted FSE plus USPIO-enhanced images $\mathrm{T}_{2}{ }^{*}$-weighted images. On T2 FSE images the short and long axis of the node, border irregularity and homogenous signal intensity were used as criteria for nodal prediction, as previously defined in literature ${ }^{14,15}$. On USPIO-enhanced $\mathrm{T}_{2}{ }^{*}$-weighted images the radiologists used the estimated percentage of the white region within the node ${ }^{13}$. When the white region within the node was estimated larger than $30 \%$, the node was staged as $\mathrm{N}+$.

\section{Surgical procedure and histological evaluation}

129 patients underwent a standard total mesorectal excision and 21 with very small tumors underwent a local excision with the Transanal Endoscopic Microsurgical (TEM) technique. The histological examination of the TME specimen was standardized: it was fixed in formalin for $24-48 \mathrm{~h}$ after the circumferential resection plane was inked. The specimen was then sectioned transversely every $5 \mathrm{~mm}$ perpendicular to the mesorectum. Careful examination was performed of the circumferential margin for involvement, measuring the shortest distance to the tumor, both macroscopically and microscopically on haematoxylin-eosin (HE) stained sections. The depth of invasion into the bowel wall and the assessment of the lymph nodes were performed according to a standard protocol on HE sections. The pathologist reported his findings concerning T-stage, CRM and nodal status blinded for the radiological predictions. All rectal specimens resected by TEM were examined for cancer invasion depth within the different layers of the bowel wall, and the specimens were evaluated whether the margins were free. Because with a TEM procedure the mesorectum is not removed and histological information on lymph nodes is lacking, patients underwent a second USPIO MRI 3-6 months and a third USPIO MRI 12 months after their TEM procedure to provide a surrogate standard reference for the lymph node status instead of postoperative histology. 


\section{Statistical analysis}

The majority of patients with an involved or close resection margin on MRI received neoadjuvant chemoradiation and were excluded from the present study. For the MR assessment of the CRM only the NPV can therefore be meaningfully evaluated. For the detection of tumors limited to the bowel wall and the detection of the nodal status the accuracy, sensitivity, specificity, and positive and negative predictive values of were calculated. For the prediction of the nodal status ROC curves were constructed and Areas under the curve (AUC's) were calculated. For the MR prediction of Stage I disease, a combination of $\mathrm{T}$ and $\mathrm{N}$ prediction, the accuracy, sensitivity, specificity, and positive and negative predictive values were calculated.

Comparisons between conventional and USPIO enhanced MRI, between the expert reader and the general radiologists, and between 1.0T and $1.5 \mathrm{~T}$ were made using diagnostic odds ratio analyses and the Delong, Delong and ClarkePearson method where appropriate ${ }^{16}$. To determine the interobserver agreement, square weighted kappa's ${ }^{17,18}$ were calculated with a $95 \%$-Confidence Interval.

The kappa and odds ratio analyses were performed by using STATA software (Stata Statistical Software: Release 9. College Station, TX: StataCorp LP). All other analyses were performed using a statistical software program (SPSS, version 12.0.1, 2003; SPSS, Chicago, Ill.).

\section{RESULTS}

During this study all patients tolerated the USPIO contrast agent well and no serious adverse events were reported. The quality of the 1.0T MR images was assessed 'poor' by the non-experts in $50 \%$ of the patients, while $1.5 \mathrm{~T}$ MRI had 'poor' MR image quality in only $12 \%$ of the patients.

87 of the eligible 150 patients were staged as pT1-2 tumors at the histopathological examination, 61 had $T_{3}$ tumors and 2 patients had a $T_{4}$ tumor. In 49 patients the histological examination revealed malignant nodes. This resulted in 71 patients with Stage I disease, 30 patients with Stage II disease and 49 patients with at least Stage III disease at histopathological examination. Six 


\section{Chapter 5}

patients had an involved/close CRM at the histological examination. At the histological examination an average of 10.7 nodes were found per patient.

\section{Accuracy of MRI for predicting CRM}

Almost all patients with an anticipated positive CRM on MR imaging receive neoadjuvant chemoradiation, therefore only the NPV can be calculated. In $148 / 150$ patients the MR had predicted a CRM of more than $2 \mathrm{~mm}$. In the two patients with a predicted involved margin a palliative resection was performed without a long course of chemoradiation, and this resulted in an involved margin in both patients. Of the 148 patients with a predicted free CRM 4 patients had an involved margin at histology. This was the result of errors in surgical technique in all 4 patients, as the histological examination showed a ruptured specimen at the level of the tumor (3) or a defect in the mesorectum (1).

These four patients should therefore not be considered as false negative MR findings. This results in a NPV of $100 \%(148 / 148)$, without any difference between 1.0T \& 1.5T MR machines and for expert and general radiologists.

70 Accuracy of MRI for predicting tumors limited to the bowel wall ( $\left.T_{1}-2\right)$

Table 5.2 shows the accuracy, sensitivity, specificity and PPV and NPV. The high PPV of 0.95 for detection of a tumor limited to the bowel wall $\left(T_{1-2}\right)$ for experts, and 0.87 for general radiologists using $1.5 \mathrm{~T}$ MRI are clinically useful. Using 1.0T MRI the expert and general radiologists predicted a tumor limited to the bowel wall with a disappointing PPV of 0.75 and 0.73 , respectively.

\begin{tabular}{lcccc}
\hline & \multicolumn{2}{c}{ 1.0T MRI } & \multicolumn{2}{c}{ 1.5T MRI } \\
PT1-2 & General Setting & University Setting & General Setting & University Setting \\
\hline ACC & 0.70 & 0.65 & 0.74 & 0.82 \\
& $((11+3) / 20)$ & $((9+4) / 20)$ & $((13+18) / 42)$ & $((53+53) / 130)$ \\
\hline Sens & 0.85 & 0.69 & 0.59 & 0.72 \\
& $(11 /(11+2))$ & $(9 /(9+4))$ & $(13 /(13+9))$ & $(53 /(53+21))$ \\
\hline Spec & 0.43 & 0.57 & 0.90 & 0.95 \\
& $(3 /(3+4))$ & $(4 /(4+3))$ & $(18 /(18+2))$ & $(53 /(53+3))$ \\
\hline PPV & 0.73 & 0.75 & 0.87 & 0.95 \\
& $(11 /(11+4))$ & $(9 /(9+3))$ & $(13 /(13+2))$ & $(53 /(53+3))$ \\
\hline NPV & 0.60 & 0.50 & 0.67 & 0.72 \\
& $(3 /(3+2))$ & $(4 /(4+4))$ & $(18 /(18+9))$ & $(53 /(53+21))$ \\
\hline
\end{tabular}

Table 5.2 shows the Accuracy (acc), sensitivity (sens), specificity (spec), PPV and NPV for tumors limited to the bowel wall for $1.0 T$ and $1.5 T$ MRI 
Accuracy of MRI for predicting lymph node involvement

At 1.5 T MRI the AUCs for the prediction of the nodal status on USPIO enhanced images and T2 FSE images for the expert were 0.92 and 0.84 , and for the general radiologists 0.87 and 0.81 respectively. The AUCS for nodal status using USPIO enhanced images and T2 FSE images at 1.OT MRI for the expert were 0.68 and 0.58 , and for general radiologists 0.72 and 0.51 respectively (see figure $5.2 \mathrm{a} \& \mathrm{~b}$ ).
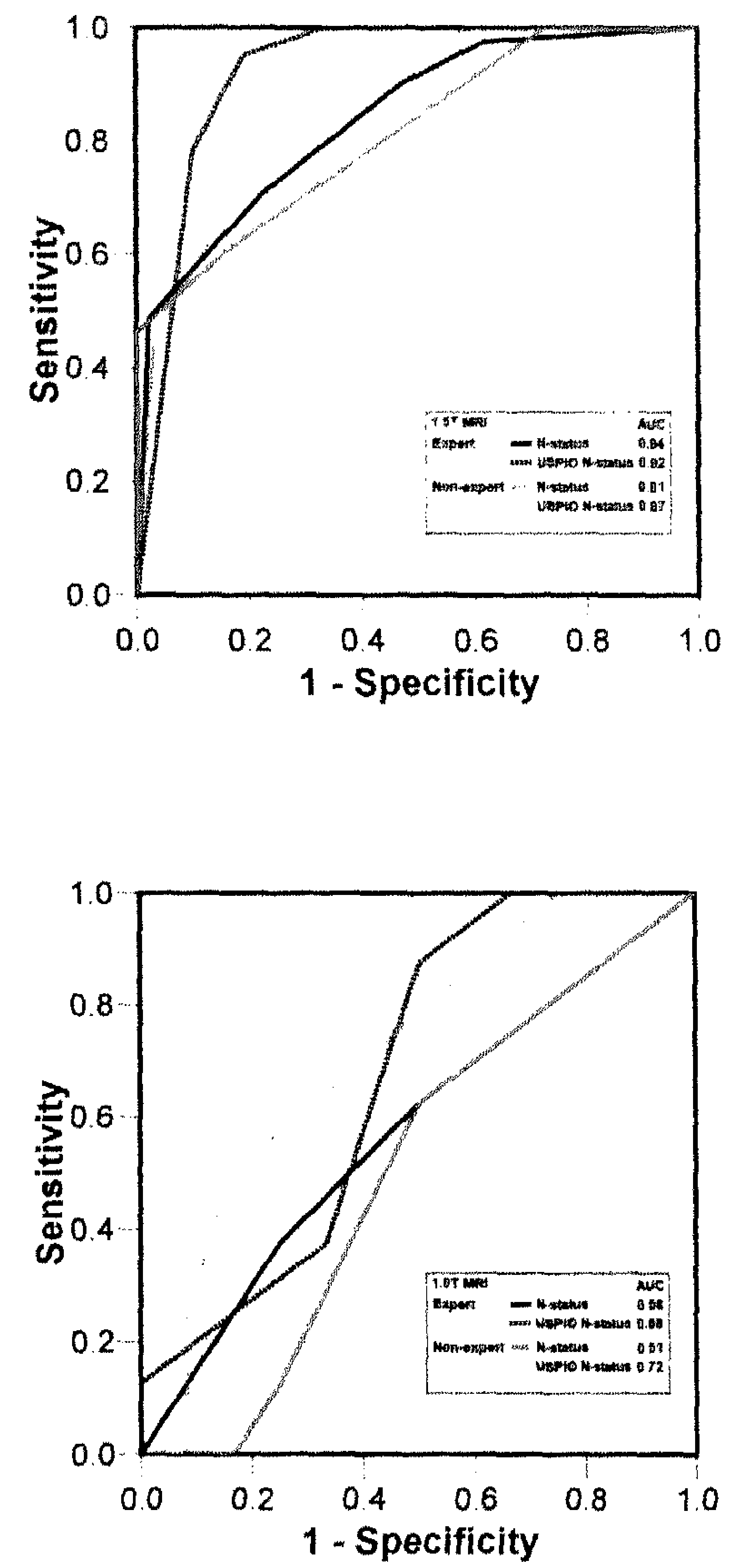

Figure 5.2a Recewer operating characteristic $\{R O C$ ) curves and areat under the curve (AUC) for expert and general radiologists, for the detection of malignant lymph nodes using non. enhanced and USPIO-entranced 3.5 TMRI

Figure $5.2 b$ Receiver operating characteristic (ROC) curves and areas under the curve (AUC) for expert and general radiologists, for the detection of malignant lymph nodes using non. enhanced and USPIO-enhanced 1.oT MRI 
Chapter 5

Table 5.3a\&b show the corresponding sensitivity, specificity, PPV and NPV using $T_{2}$ FSE images and USPIO-enhanced prediction. The expert and general radiologist both improved their prediction using USPIO-enhanced MR images. For the general radiologists this improvement was statistically significantly for 1.0T $(P=0.024)$ and not 1.5T MRI $(P=0.331)$, as opposed to the expert radiologist where the improvement was significant for $1.5 T M R I(P=0.010)$ and not 1.0T MRI $(P=0.430)$. Overall $1.5 T M R I$ showed a positive trend for improved results for nodal staging with USPIO compared to $1.0 T(P=0.060)$.

\begin{tabular}{lcccc}
\hline Detection of N+ & \multicolumn{2}{c}{ Non-enhanced MRI } & \multicolumn{2}{c}{ USPIO MRI } \\
1.5T MRI & General Setting & University Setting & General Setting & University Setting \\
\hline AUC & 0.81 & 0.84 & 0.87 & 0.92 \\
$95 \% \mathrm{Cl}$ & $(0.67-0.96)$ & $(0.77-0.91)$ & $(0.76-0.98)$ & $(0.88-0.97)$ \\
\hline Sens & 0.62 & 0.90 & 0.92 & 0.95 \\
& $(8 /(8+5))$ & $(37 /(37+4))$ & $(12 /(12+1))$ & $(39 /(39+2))$ \\
\hline SpeC & 0.83 & 0.53 & 0.69 & 0.81 \\
& $(24 /(24+5))$ & $(47 /(47+42))$ & $(20 /(20+9))$ & $(72 /(72+17))$ \\
\hline PPV & 0.62 & 0.47 & 0.57 & 0.70 \\
& $(8 /(8+5))$ & $(37 /(37+42))$ & $(12 /(12+9))$ & $(39 /(39+17))$ \\
\hline NPV & 0.83 & 0.92 & 0.95 & 0.97 \\
& $(24 /(24+5))$ & $(47 /(47+4))$ & $(20 /(20+1))$ & $(72 /(72+2))$ \\
\hline
\end{tabular}

Table 5.3a shows the area under the ROC curve (AUC) and the corresponding sensitivity (sens), specificity (spec), PPV and NPV for the non-enhanced and USPIO-enhanced prediction of the nodal status for $1.5 T$ MRI

\begin{tabular}{lcccc}
\hline Detection of + & \multicolumn{2}{c}{ Non-enhanced MRI } & \multicolumn{2}{c}{ USPIO MRI } \\
1.0T MRI & General Setting & University Setting & General Setting & University Setting \\
\hline AUC & 0.51 & 0.58 & 0.72 & 0.68 \\
$95 \% \mathrm{Cl}$ & $(0.25-0.76)$ & $(0.32-0.84)$ & $(0.49-0.95)$ & $(0.44-0.91)$ \\
\hline Sens & 0.63 & 0.63 & 0.50 & 0.88 \\
& $(5 /(5+3))$ & $(5 /(5+3))$ & $(4 /(4+4))$ & $(7 /(7+1))$ \\
\hline Spec & 0.50 & 0.50 & 0.83 & 0.50 \\
& $(6 /(6+6))$ & $(6 /(6+6))$ & $(10 /(10+2))$ & $(6 /(6+6))$ \\
\hline PPV & 0.45 & 0.45 & 0.67 & 0.62 \\
& $(5 /(5+6))$ & $(5 /(5+6))$ & $(4 /(4+2))$ & $(7 /(7+6))$ \\
\hline NPV & 0.67 & 0.67 & 0.71 & 0.77 \\
& $(6 /(6+3))$ & $(6 /(6+3))$ & $(10 /(10+4))$ & $(6 /(6+1))$ \\
\hline
\end{tabular}

Table 5.36 shows the area under the ROC curve (AUC) and the corresponding sensitivity (sens), specificity (spec), PPV and NPV for the non-enhanced and USPIO-enhanced prediction of the nodal status for $1.0 T$ MRI 


\section{Stage / disease}

Table 5.4 shows the accuracy, sensitivity, specificity, PPV and NPV for the prediction of Stage I disease. The clinically relevant PPV for experts and general radiologists using $1.5 \mathrm{~T}$ USPIO MRI was 0.94 and 0.92 respectively. Using $1.0 \mathrm{~T}$ USPIO MRI the expert and general radiologists predicted Stage I disease with a lower PPV of 0.80 and 0.64 , respectively.

\begin{tabular}{lcccc}
\hline \multirow{2}{*}{ Stage 1 disease } & \multicolumn{2}{c}{ 1.0T MRI } & \multicolumn{2}{c}{ 1.5T MRI } \\
Acc & General Setting & University Setting & General Setting & University Setting \\
\hline Sens & 0.70 & 0.70 & 0.79 & 0.85 \\
& $((7+7) / 20)$ & $((4+10) / 20)$ & $((12+21) / 42)$ & $((45+65) / 130)$ \\
\hline Spec & 0.78 & 0.44 & 0.60 & 0.73 \\
& $(7 /(7+2))$ & $(4 /(4+5))$ & $(12 /(12+8))$ & $(45 /(45+17))$ \\
\hline PPV & 0.64 & 0.91 & 0.95 & 0.96 \\
& $(7 /(7+4))$ & $(10 /(10+1))$ & $(21 /(21+1))$ & $(65 /(65+3))$ \\
\hline NPV & 0.64 & 0.80 & 0.92 & 0.94 \\
& $(7 /(7+4))$ & $(4 /(4+1))$ & $(12 /(12+1))$ & $(45 /(45+3))$ \\
\hline Table 5-4 Accuracy (acd) sensitivity $($ sens) specificity $($ spec) PPV and NPV for Stage 1 disease for 1.5Tand 1.0T MRI
\end{tabular}

\section{Interobserver agreement}

The interobserver agreement between the general and expert MR radiologist for predicting tumors limited to the bowel wall was good $(K=0.70)$ using $1.5 T M R I$ and moderate $(K=0.44)$ using 1.0T MRI (see table 5.5).

\begin{tabular}{lcc}
\hline Interobserver agreement (Kappa) & 1.0T MRI & 1.5T MRI \\
\hline T1-2 & 0.44 & 0.70 \\
USPIO N-status & 0.02 & 0.71 \\
Stage 1 disease & 0.05 & 0.74 \\
\hline Table 5.5 Interobserver agreements for the prediction of the tumors, nodal status and Stage 1 disease by expert and \\
non-expert radiologist using 1.oT and 1.5T USPIO MRI
\end{tabular}

Using 1.5T MRI the interobserver agreement between the expert and the general radiologist MRI for the USPIO-enhanced prediction of the nodal status was substantial $(K=0.71)$, whereas a slight $(K=0.02)$ agreement was found using $1.0 T$ MRI.

The interobserver agreement between the general and expert MR radiologist for predicting stage I disease was substantial $(K=0.74)$ using $1.5 T \mathrm{MRI}$ and only slight 


\section{Chapter 5}

agreement $(K=0.05)$ was found using 1.0T MRI. There was no disagreement between expert and general radiologists concerning the CRM prediction.

\section{DISCUSSION}

This multicenter study shows that expert and general radiologists could accurately detect a noninvolved CRM as demonstrated by the high NPV. A tumor that is limited to the bowel wall could also be accurately predicted with a high PPV using 1.5T MRI by all radiologists. This study also proved that both expert and general radiologists showed a high NPV for predicting malignant nodes on USPIO enhanced 1.5T MR images. Furthermore experts and general radiologists could accurately detect Stage I disease with a high PPV using 1.5T USPIO MRI.

\section{Accuracy of MRI for CRM}

This multicenter study demonstrated a high negative predictive value for the prediction of an involved or close $(<2 \mathrm{~mm})$ anticipated CRM at the mesorectal fascia. Many studies have shown that a close resection margin is a very important prognostic indicator for local recurrence ${ }^{19-21}$. Quirke et $\mathrm{al}^{20}$ have demonstrated that microscopically positive resection margins resulted in a local recurrence rate of $83 \%$. Even with a short course of preoperative radiotherapy is insufficient to prevent local recurrences, and these patients should be treated with a long course of chemoradiation ${ }^{22}$. So far MRI is the only modality that has been shown to be accurate for the prediction of the relation of the tumor to the mesorectal fascia. Many single center studies ${ }^{23}$, confirmed by the multicenter Mercury trial ${ }^{24}$, and again confirmed in this study. In the design of the present study a confirmation of the ability to image an involved mesorectal fascia was not possible, as these patients went on to receive a long course of chemoradiation, and thereby loosing histology as the reference standard.

Accuracy of MRI for predicting tumors limited to the bowel wall

Expert and general radiologists accurately predicted a tumor limited to the bowel wall with a high PPV of 0.95 and 0.87 respectively. Although a clear distinction between $\mathrm{pT}_{1}$ and $\mathrm{pT}_{2}$ cannot be made on T2W TSE images, the distinction between a $T_{2}$ and $T_{3}$ tumor appears easier to make on MR. The criterion of an 
intact rectal wall seems to be a highly predictive sign for pT1-2 tumors (see figure 5.3a). The low NPV was due to considerable overstaging in borderline $\mathrm{PT} 2$ tumors with strandlike desmoplastic reactions in the perirectal fat (see figure $5.3 \mathrm{~b}$ ). A significant proportion of the overstaging errors occurred especially with less experienced radiologists. Overstaging problems have been well documented, with reports of overstaging of $38-62 \%$ of $p T_{2}$ tumors as $T_{3}$ tumors by several studies ${ }^{23,25}$. Although EUS is better in distinguishing the individual layers of the bowel wall, it is also known to have the same limitation in distinguishing between $\mathrm{pT} 2$ and $\mathrm{pT} 3$ with overstaging errors of up to $36 \%$ in pT 2 patients ${ }^{26}$.
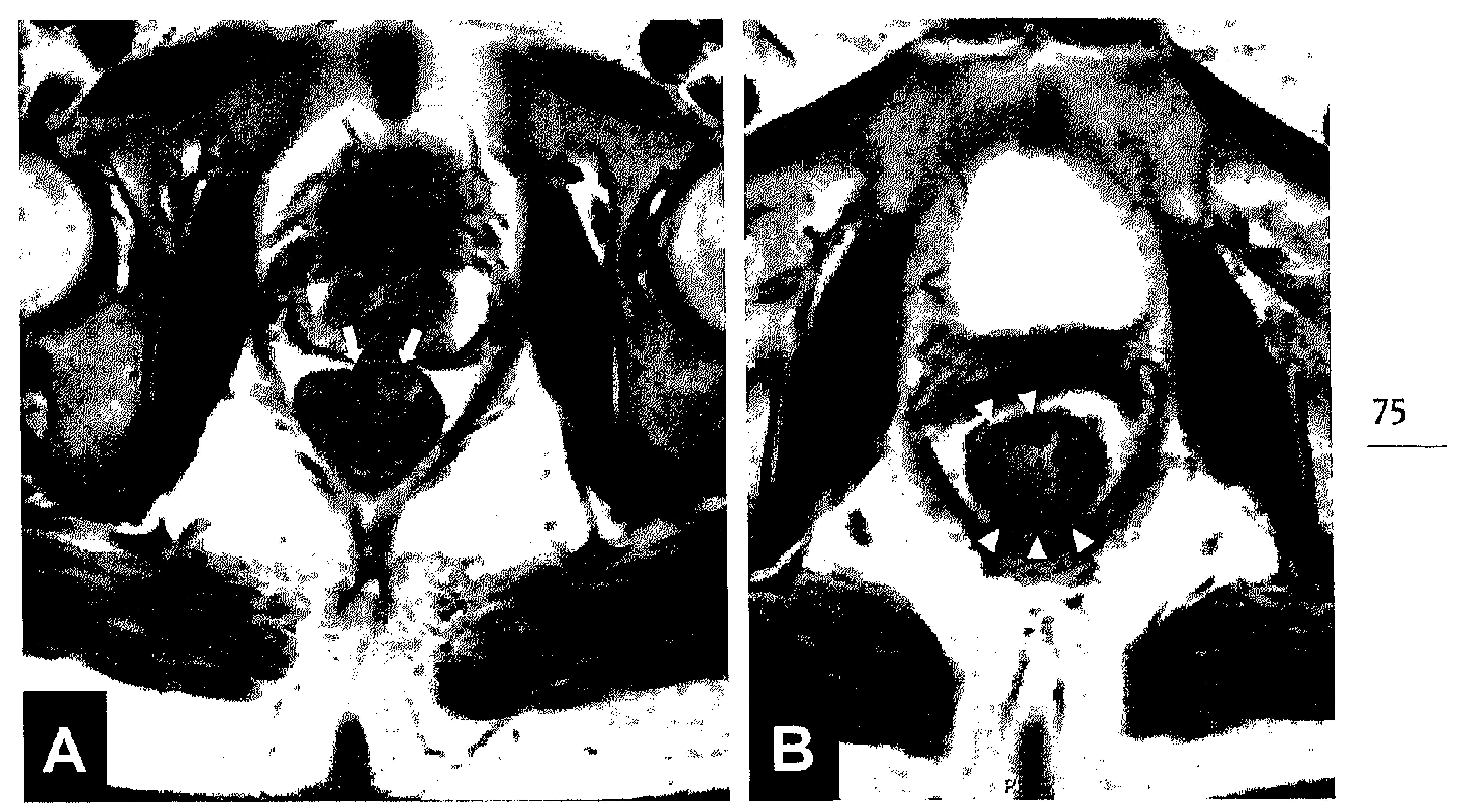

Figure 5.3a/b. (A) Axial T2W TSE MR image shows a distal tumor in which the rectal wall (arrows) is intact, therefore this was correctly staged as a T2 tumor. (B) Axial T2W TSE MR image shows another distal tumor; in this case the bowel wall is not intact with fibrotic stranding in the perirectal fat (arrowheads). Therefore the expert and general radiologist staged this as a $T_{3}$ tumor, however at histology this tumor was staged as a $T_{2}$ tumor. Due to this overstaging error the CRM was preoperatively believed to be involved and thus this patients was wrongly stratified for chemoradiation (locally advanced tumor) instead of $5 \times 5$ Gy radiotherapy (non-locally advanced tumor)

Accuracy of MRI for predicting lymph node involvement

This multicenter study demonstrated good results for prediction of the nodal status with USPIO enhanced 1.5T MRI both in a university and in general setting 


\section{Chapter 5}

(see figure $5.4 \mathrm{a} \& \mathrm{~b})$. Especially the high NPV (0.95) is promising, because it implicates that USPIO MRI could accurately select patients with a No status. We however encountered a considerable number of false positive results. There are several explanations. The design of the project was such that to avoid undertreatment, false positive findings were considered less problematic than false negatives, thereby encouraging radiologists to overstage rather than understage in doubtful cases. Secondly several benign conditions like focal granulamatous reactions or a fatty hilum can also cause a white region in the center of the node, thus mimicking a malignant node (see figure 5.5). The few false negatives that were seen were small clusters of micrometastases of less than $0.2 \mathrm{~mm}$.
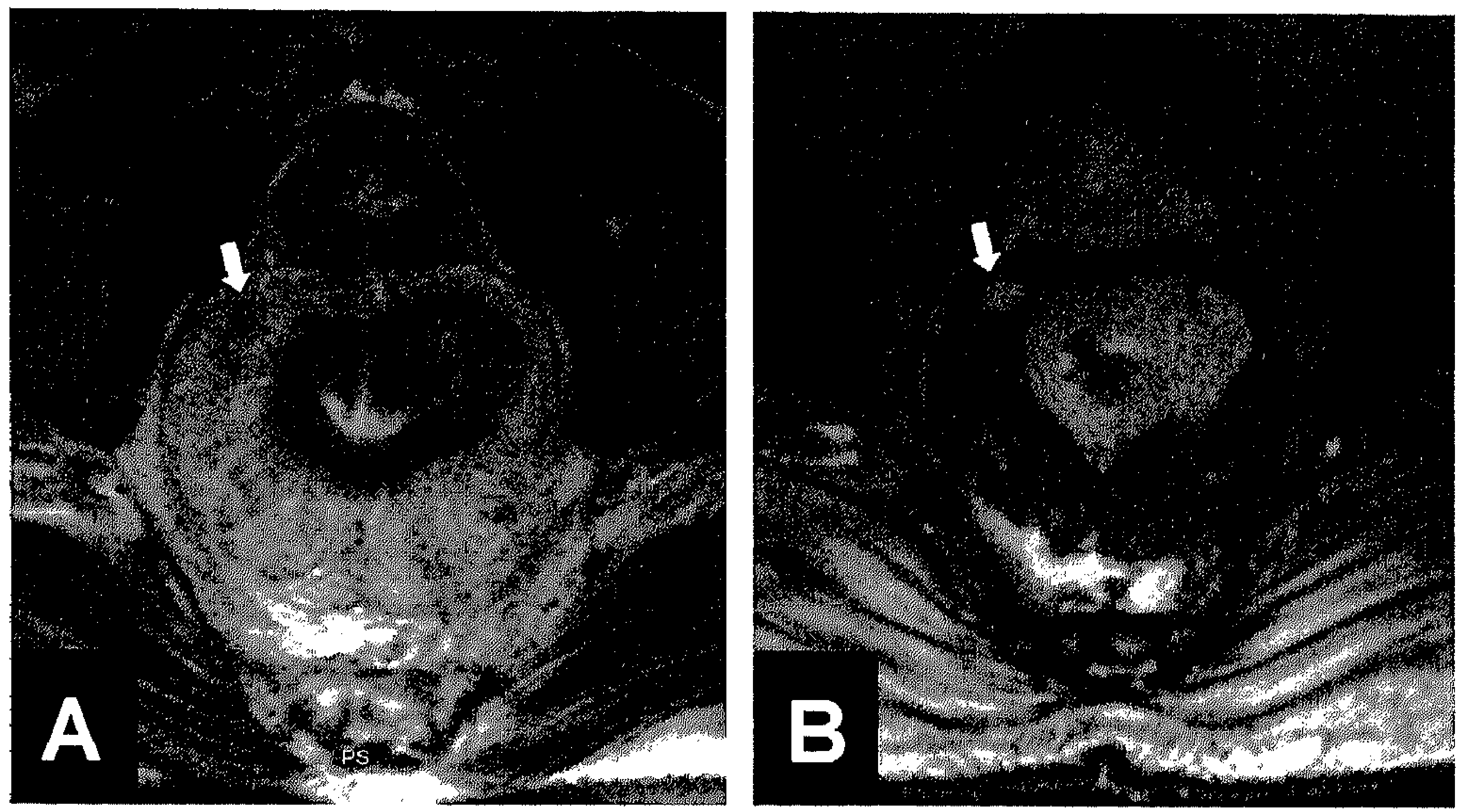

Figure 5.4a/b A $5 \mathrm{~mm}$ malignant mesorectal node, detected with USPIO MRI: (A) on T2-weighted FSE image it is difficult to differentiate between malignant and benign, $(B)$ on USPIO-enhanced $T_{2} *$ image the node has a high signal intensity, indicating that this is a malignant node. At histology this patient confirmed to have a $N_{1}$ status

The use of a lymph node specific MR contrast agent (USPIO) resulted in an improvement of AUC for all radiologists, although not all improvements were statistically significant. Overall it seems however that USPIO improves the accuracy in the order of $10 \%$. The use of morphological criteria in standard MR without contrast such as border and heterogeneous signal intensity results in a 
reasonable accuracy that is better than when only size criteria are used. This confirms earlier reports on the usefulness of these morphological criteria ${ }^{14,15}$.

A drawback of USPIO is that in Europe USPIO's application for a marketing authorization at the Committee for Medical Products for Human Use (CHMP) has recently been withdrawn by the manufacturer while in the United States the approval by the Food and Drugs Administration (FDA) is still pending. When eventually USPIO would not be marketed our studies would still have shown that lymph node staging can be improved with a lymph node specific contrast agent, stimulating the search for other reliable contrast agents or new MR techniques.
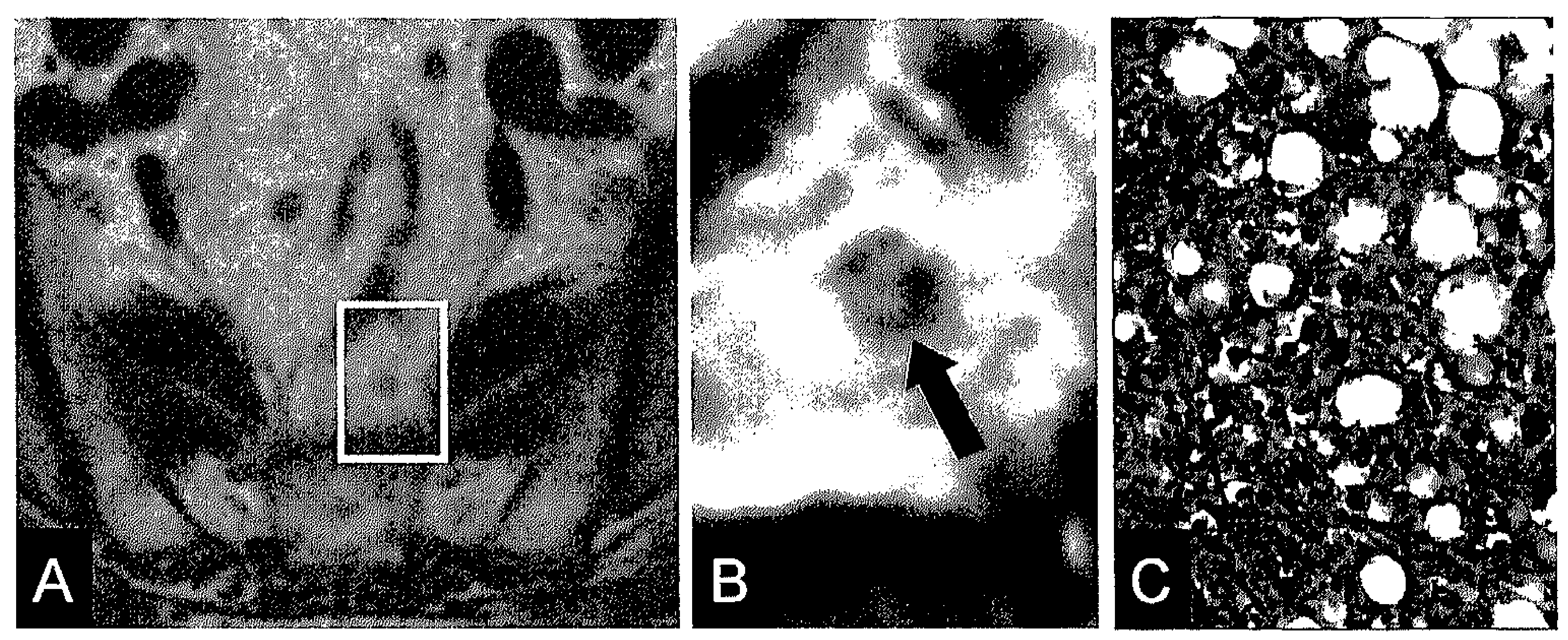

Figure 5.5a/b/c (A) T2-weighted TSE image in the axial plane of the pelvis with a $5 \mathrm{~mm}$ mesorectal lymph node (rectangle) in a rectal cancer patient. (B) On the corresponding UPSIO-enhanced T2*-weighted image the same lymph node (arrow) showed central high signal intensity, indicating no USPIO uptake. (C) However, this correlated at histological examination (HE staining) with the centrally located granulamatous reactions in a benign node and not with tumor. This is an example of a false positive result

\section{Stage / disease}

Expert and general radiologists accurately predicted Stage I disease with a high PPV of 0.94 and 0.92 respectively. This means that radiologists can play an important role in the multidisciplinary approach of rectal cancer selecting those low risk patients who do not benefit from neoadjuvant treatment. It remains to be established whether MRI is accurate enough to allow a selection of patients who could be treated with a local excision. The lymph node metastases that have been detected in $T_{1}$ tumors are generally smaller than in $T_{2}$ or $T_{3}$ tumors, with a 
Chapter 5

higher risk of false negative MR finding ${ }^{27}$. Further clinical studies in this setting are required.

\section{Interobserver agreement}

The high interobserver agreement between expert and general radiologists for identifying $\mathrm{PT}_{1-2}$ tumors, nodal involvement at $1.5 \mathrm{~T}$ MRI and Stage I disease illustrates that MR results were consistent and reproducible, indicating that MRI staging can be done not only in the setting of an expert center but also in the setting of a general hospital.

\section{Field strength}

The results of this study show that overall there was some difference in performance for $\mathrm{T}$ - and $\mathrm{N}$-stage prediction at 1.0 Tesla compared to 1.5 Tesla, with 1.0 Tesla MRI performing less good. This difference was statistically significant for the $\mathrm{T}$ but only showed a tendency towards significance for the $\mathrm{N}$ staging. Although the total number of patients enrolled at both 1.0 Tesla centers were disproportionately lower (20/150) as compared to that enrolled at both 1.5 Tesla centers (130/150) and definite conclusions cannot be drawn, the results suggest that $\mathrm{T}$ - and $\mathrm{N}$-stage assessment in rectal cancer staging at 1.0 Tesla MR should not be relied on heavily. Because of the low field strength 1.0 Tesla MR there is an inherent inferior signal to noise ratio when trying to obtain small pixel volumes, as compared to 1.5 Tesla MR. In the balance between the best contrast and the best spatial resolution, we cannot but compromise on the spatial resolution for the 1.0 Tesla protocol. As a consequence the pixel volumes with 1.0 Tesla MRIs for the $2 \mathrm{D} \mathrm{T} \mathrm{T}_{2} \mathrm{~W}, 3 \mathrm{D} \mathrm{T} \mathrm{T}_{1} \mathrm{~W}$ and $3 \mathrm{D} \mathrm{T}_{2} * \mathrm{~W}$ sequences are 7,3 and 3.7 times larger as with the 1.5 Tesla MRI. This could well explain the difference in subjective interpretation of 'good' and 'poor' image quality, and the lower performance of $\mathrm{T}$ - and $\mathrm{N}$-staging with 1.0 $\mathrm{T}$. The assessment of the anticipated circumferential margin did not appear to suffer from this limitation.

\section{Conclusion}

This multicenter study shows that radiologists can accurately predict a noninvolved CRM with MR imaging. Furthermore USPIO MRI at $1.5 \mathrm{~T}$ can accurately predict tumors limited to the bowel wall with a high PPV. USPIO MRI 
at 1.5T can also accurately select pNo patients with a high NPV for the prediction of malignant nodes. The performance of an experienced and less experienced radiologist were equal, indicating that preoperative selection with MRI can also be reliable in the setting of a general hospital. 


\section{Chapter 5}

\section{REFERENCES}

1.

Parkin DM, Bray F, Ferlay J, Pisani P. Estimating the world cancer burden: Globocan 2000. Int J Cancer 2001;94(2): 153-156.

Jemal A, Tiwari RC, Murray T, Ghafoor A, Samuels A, Ward E, Feuer EJ, Thun MJ. Cancer statistics, 2004. CA Cancer J Clin 2004;54(1): 8.29.

Boyle P, Ferlay J. Cancer incidence and mortality in Europe, 2004. Ann Oncol 2005;16(3): 481-488.

Brown $G$, Daniels IR. Preoperative staging of rectal cancer: the MERCURY research project. Recent Results Cancer Res 2005;165: 58-74.

Pahlman L, Glimelius B. Pre- or postoperative radiotherapy in rectal and rectosigmoid carcinoma. Report from a randomized multicenter trial. Ann Surg 1990;211(2): 187-195.

Improved survival with preoperative radiotherapy in resectable rectal cancer. Swedish Rectal Cancer Trial. N Engl J Med 1997;336(14): 980-987.

Kapiteijn E, Marijnen CA, Nagtegaal ID, Putter $H$, Steup WH, Wiggers T, Rutten HJ, Pahlman $L$, Glimelius $B$, van Krieken JH, Leer JW, van de Velde CJ. Preoperative radiotherapy combined with total mesorectal excision for resectable rectal cancer. N Engl J Med 2001;345(9): 638-646.

Bipat S, Glas AS, Slors FJ, Zwinderman AH, Bossuyt PM, Stoker J. Rectal cancer: local staging and assessment of lymph node involvement with endoluminal US, CT, and MR imaging--a meta-analysis. Radiology 2004;232(3): 773-783. of nanoparticle-enhanced MRI for lymph-node metastases: a meta-analysis. Lancet Oncol 2006;7(1): 52-6o.

Marijnen CA, Nagtegaal ID, Klein Kranenbarg E, Hermans J, van de Velde CJ, Leer JW, van Krieken $\mathrm{JH}$. No downstaging after short-term preoperative radiotherapy in rectal cancer patients. J Clin Oncol 2001;19(7): 1976-1984.

Weissleder R, Elizondo $G$, Wittenberg J, Rabito CA, Bengele HH, Josephson L. Ultrasmall superparamagnetic iron oxide: characterization of a new class of contrast agents for MR imaging. Radiology 1990;175(2): 489*493.

Frija G, Clement O, Le Guen O, Cuenod CA, Siauve N, Benderbous S. Experimental investigation of the delivery pathway of ultrasmall superparamagnetic iron oxide to lymph nodes. Acad Radiol 1996;3 Suppl 2: 5299-300.

Lahaye MJ, Engelen SM, Kessels AG, de Bruine AP, von Meyenfeldt MF, van Engelshoven JM, van de Velde CJ, Beets GL, Beets-Tan RG. USPIO-enhanced MR Imaging for Nodal Staging in Patients with Primary Rectal Cancer: Predictive Criteria. Radiology 2008.

Brown G, Richards CJ, Bourne MW, Newcombe RG, Radcliffe AG, Dallimore NS, Williams GT. Morphologic Predictors of Lymph Node Status in Rectal Cancer with Use of High-Spatial-Resolution MR Imaging with Histopathologic Comparison. Radiology 2003;227(2): 371-377.

Kim JH, Beets GL, Kim MJ, Kessels AG, Beets-Tan RG. High-resolution MR imaging for nodal staging in rectal cancer; are there any criteria in addition to the size? Eur J Radiol 2004i52(1): 78-83.

DeLong ER, D. M. DeLong, and D. L. Clarke-Pearson. . Comparing the areas under two or more correlated receiver operating curves: A nonparametric approach. . Biometrics 1988;44: 837-845

Cohen J. A coefficient of agreement for nominal scales. Educational and Psychological Measurement 1960;20: 37-46.

Cohen J. Weighted Kappa: nominal scale agreement with provision for scaled disagreement or partial credit. Psychol Bull 1968;70: 213-230.

Adam IJ, Mohamdee MO, Martin IG, Scott N, Finan PJ, Johnston D, Dixon MF, Quirke P. Role of circumferential margin involvement in the local recurrence of rectal cancer, Lancet 1994i344(8924): 707-711.

Quirke $P$, Durdey $P$, Dixon MF, Williams NS. Local recurrence of rectal adenocarcinoma due to inadequate surgical resection. Histopathological study of lateral tumour spread and surgical excision. Lancet 1986;2(8514): 996-999. 


\section{MR Prediction of CRM, T- and N-stage}

21.

22.

23

24 .

25 .

26.

27 .

Birbeck KF, Macklin CP, Tiffin NJ, Parsons W, Dixon MF, Mapstone NP, Abbott CR, Scott N, Finan PJ, Johnston $D$, Quirke $P$. Rates of circumferential resection margin involvement vary between surgeons and predict outcomes in rectal cancer surgery. Ann Surg 2002;235(4): 449-457.

Marijnen CA, Nagtegaal ID, Kapiteijn E, Kranenbarg EK, Noordijk EM, van Krieken JH, van de Velde CJ, Leer JW. Radiotherapy does not compensate for positive resection margins in rectal cancer patients: report of a multicenter randomized trial. Int J Radiat Oncol Biol Phys 2003;55(5): 1311-1320.

Beets-Tan RG, Beets GL, Vliegen RF, Kessels AG, Van Boven H, De Bruine A, von Meyenfeldt MF, Baeten $C G$, van Engelshoven JM. Accuracy of magnetic resonance imaging in prediction of tumourfree resection margin in rectal cancer surgery. Lancet 2001;357(9255): 497-504.

Strassburg J. Magnetic resonance imaging in rectal cancer: the MERCURY experience. Tech Coloproctol 2004;8 Suppl 1: 516-18.

Poon FW, McDonald A, Anderson JH, Duthie F, Rodger C, McCurrach G, McKee RF, Horgan PG, Foulis $A K$, Chong $D$, Finlay IG. Accuracy of thin section magnetic resonance using phased-array pelvic coil in predicting the T-staging of rectal cancer. Eur J Radiol 2005;53(2): 256-262.

Marusch F, Koch A, Schmidt U, Zippel R, Kuhn R, Wolff S, Pross M, Wierth A, Gastinger I, Lippert H. Routine use of transrectal ultrasound in rectal carcinoma: results of a prospective multicenter study. Endoscopy 2002;34(5): 385-390.

Landmann RG, Wong WD, Hoepfl J, Shia J, Guillem JG, Temple LK, Paty PB, Weiser MR. Limitations of early rectal cancer nodal staging may explain failure after local excision. Dis Colon Rectum 2007;50(10): 1520-1525. 


\section{MRI-based tailored treatment of rectal cancer: impact on surgical outcome}

S.M.E. Engelen, M.J. Lahaye, A.G.H. Kessels, J.W.A. Leiftens, C.L.H. van Berlo, H. Verkooijen, R.L.H. Jansen,

G. Lammering, M.F. von Meyenfeldt, C.J.H. van de Velde, R.G.H. Beets-Tan, G.L. Beets 
Chapter 6

\section{ABSTRACT}

Aim The purpose of this multicenter cohort study was to evaluate whether tailormade treatment of primary rectal cancer based on MRI can reduce the number of incomplete resections, and improve recurrence-free and overall survival. This study cohort has been compared with an historical control in which no standardized preoperative imaging was used.

Methods From February 2003 until January 2008296 patients were enrolled. All underwent preoperative MRI using a lymph node specific contrast agent (USPIO) to predict CRM, T- and $\mathrm{N}$-stage. Based on expert reading of the MRI, patients were stratified in different risk groups for local recurrence: (a) low risk (wide CRM and No status), (b) intermediate risk and (c) high risk (close/involved CRM, N2 status or distal tumors). Mainly based on this MRI risk assessment patients were treated with (a) surgery only (TME or local excision), (b) preoperative $5 \times 5 \mathrm{~Gy}+$ $\mathrm{TME}_{\text {, }}$ and (c) a long course of chemoradiation therapy followed by surgery after a 6-8 week interval. The number of complete resections (CRM $>1 \mathrm{~mm}$ ) was determined by histopathological evaluation of the resection specimen.

Results There were 228 patients that underwent treatment with curative intention: 49 with surgery only, 86 with $5 \times 5$ Gy and surgery and 93 with chemoradiation and surgery. The number of complete resections was 218 (95.6\%), which is a statistically significant improvement $(p<0.001)$ compared to the historical control ( $84 \%$ ). The median follow up is 18.7 months. The actuarial 2 years local recurrence rate is $2.8 \%$ and overall survival is $86.5 \%$.

Conclusion Tailored treatment of rectal cancer based on dedicated preoperative MR imaging has increased the number of microscopically complete resections significantly as compared to a historical control. The advantage of tailored treatment consists of the avoidance of over- and undertreatment in patients with different risk for local recurrence. Longer follow up is required to confirm whether the improvement in complete resections will indeed lead to a better clinical outcome. 


\section{INTRODUCTION}

In the past decades, studies have shown that the risk for recurrence after resection of rectal cancer is substantially reduced with the surgical technique of the Total Mesorectal Excision (TME), popularized by Heald, in which the tumor including the surrounding mesorectal fat, containing lymph nodes, is removed as a complete package ${ }^{1,2}$. Additionally, neoadjuvant (chemo)radiation has proved to improve local control, and in some studies survival ${ }^{3-6}$. Large randomized trials are powerful tools to prove the effectiveness of a therapy when small differences are clinically important. The large numbers of patients that are required to provide the necessary power often result in broad inclusion criteria and little or no stratification for patient or tumor characteristics. This is at odds with the clinical concept of individualization of treatment, in which the treatment is adapted to the individual patient taking into account characteristics such as age, comorbidity, stage and location of the tumor etc. Although individualization of treatment is expected to provide an optimal balance between minimizing treatment related morbidity and best oncological outcome, there is no hard proof that the outcome is better than applying a single standard treatment for all patients. Subgroup analyses within the large randomized trials can provide clues as to what factors can be used to guide treatment decisions for individual patients. In most of the trials patients with stage I disease ( $\mathrm{T}_{1-2 \mathrm{No}}$ ) have a negligible risk for local recurrence, and therefore do not need preoperative irradiation 7 . On the other hand, in patients with a combination of unfavorable characteristics like a tumor extending into the mesorectal fascia, positive lymph nodes and a very distal location, a short course of preoperative radiation does not provide enough protection against local recurrence, and a long course of preoperative chemoradiation is required ${ }^{3,4,7}$.

Reliable preoperative imaging is essential for a differentiated treatment according to risk factors for local recurrence. Although endorectal ultrasound is good in assessing the extent of the primary tumor in small lesions ${ }^{8}$, MRI has repeatedly been shown to provide the best information on the relation of the tumor to the mesorectal fascia ${ }^{9-13}$. Assessing nodal involvement however has been suboptimal, and up till now all three imaging modalities (EUS,CT and MRI) lack accuracy sufficient for important clinical decision-making ${ }^{14}$. Recently MRI 


\section{Chapter 6}

studies with lymph node-specific contrast agents have shown promising results for prediction of nodal involvement ${ }^{15,16}$. This would enable MRI to assess the two most important risk factors for local recurrence: relation of the tumor to the mesorectal fascia and nodal stage.

The aim of our study was to assess the outcome as defined by the number of complete resections of a differentiated treatment protocol for rectal cancer, based on MRI. The results are compared to a historical control, the Dutch TME trial, in which patients were randomized between a short course of preoperative radiotherapy or no neoadjuvant therapy, and in which no standardized preoperative imaging was used?

\section{METHODS}

\section{Patients}

Between February 2003 and January 2008 we have performed a prospective multicenter project with a cohort of patients with primary rectal cancer in whom a differentiated treatment protocol was primarily based on MRI with USPIO. In February 2003 the study started as a single centre pilot study in the University Hospital Maastricht, and was continued as a multicenter study from December 2005 onwards. Three regional hospitals joined the study: Laurentius Hospital Roermond (start of inclusion: 12-2005 ), St. Jans Hospital Weert (start of inclusion: 12-2005) and VieCuri Medical Center Venlo (start of inclusion: 022006). Institutional review board approval was obtained for all hospitals. All patients gave a written informed consent.

Inclusion criteria: Histologically proven adenocarcinoma of the rectum.

Exclusion criteria: Patients were excluded from the study when they were pregnant, had an age below 18 years, a contra-indication for MR imaging (pacemaker, neurostimulator, insulin pump, certain vascular clips (e.g. used in brain surgery), cochlear implants, metallic splinters in the eye or any other metal implant not securely fixed or electronic device), or did not give informed consent for participation.

For the present study on the outcome only patients who were treated with curative intent were selected. Patients who received palliative treatment and 
with a previous or coexisting malignancy were therefore excluded for the analyses.

\section{USPIO-MRI}

All patients underwent a pelvic MRI using Ultrasmall Super Paramagnetic Iron Oxide (a lymph node specific contrast agent) preoperatively. The USPIO MR contrast agent (Sinerem, Guerbet Laboratories, Roissy, France) consists of low molecular weight iron oxide coated with dextran. Sinerem was supplied as a powder which was reconstituted using saline and administered at a dose of 2.6 $\mathrm{mg} \mathrm{Fe} / \mathrm{kg}$. Administration took place by slow intravenous infusion within a period of 45 minutes, thirty-six to twenty-four hours before MRI scan. Imaging was performed on a 1.0/1.5T MR scanner. (See methods and table 1, chapter 5 for scan parameters). Sequences used were sagittal and axial 2D T2W TSE, axial $T_{2} * W$ GRE and axial 3D TIW GRE. Patients did not receive bowel or other preparation. Total scan time was approximately 40 minutes.

\section{Image evaluation}

All MRI scans were read by a local radiologist who predicted T-stage, $\mathrm{N}$-stage, circumferential resection margin and height of the tumor. The MRI scans were also read by an expert reader, within 3-5 days after the MRI scan was made, based on which treatment strategy was planned.

\section{Treatment stratification and strategy}

In a multidisciplinary meeting the treatment plan for each individual patient was determined based on the clinical information, MR imaging of local disease and imaging for distant disease. Patients who were proven to have widespread metastatic disease or had a very poor general condition and could not tolerate extensive treatment were designated as incurable and excluded from further analysis. On the basis of MRI patients were classified in 'low', 'intermediate', and 'high' risk for local recurrence. Low risk was defined as $T_{1-2} N_{0}$, as well as $T_{3}$ No with a wide CRM (>2 $\mathrm{mm}$ ) when localized in the proximal rectum. High risk was defined as tumors with circumferential resection margin $<2 \mathrm{~mm}^{17}$ (i.e. $1 \mathrm{~mm}$ which defines an incomplete resection (R1) $+1 \mathrm{~mm}$ MR safety margin), distal tumors or $\mathrm{N} 2$ status. All other tumors were considered as intermediate risk (see figure 6.I). 


\section{Chapter 6}

Generally the treatment advice for low risk tumors was surgery only, for the intermediate risk tumors $5 \times 5$ Gy preoperative radiotherapy and immediate surgery, and for the high risk tumors a long course of chemoradiation and surgery after a 6-8 week interval. The final decision on the treatment of an individual patient was made by the treating clinicians at the multidisciplinary meeting, were other factors such as comorbidity and performance status were taken into account.

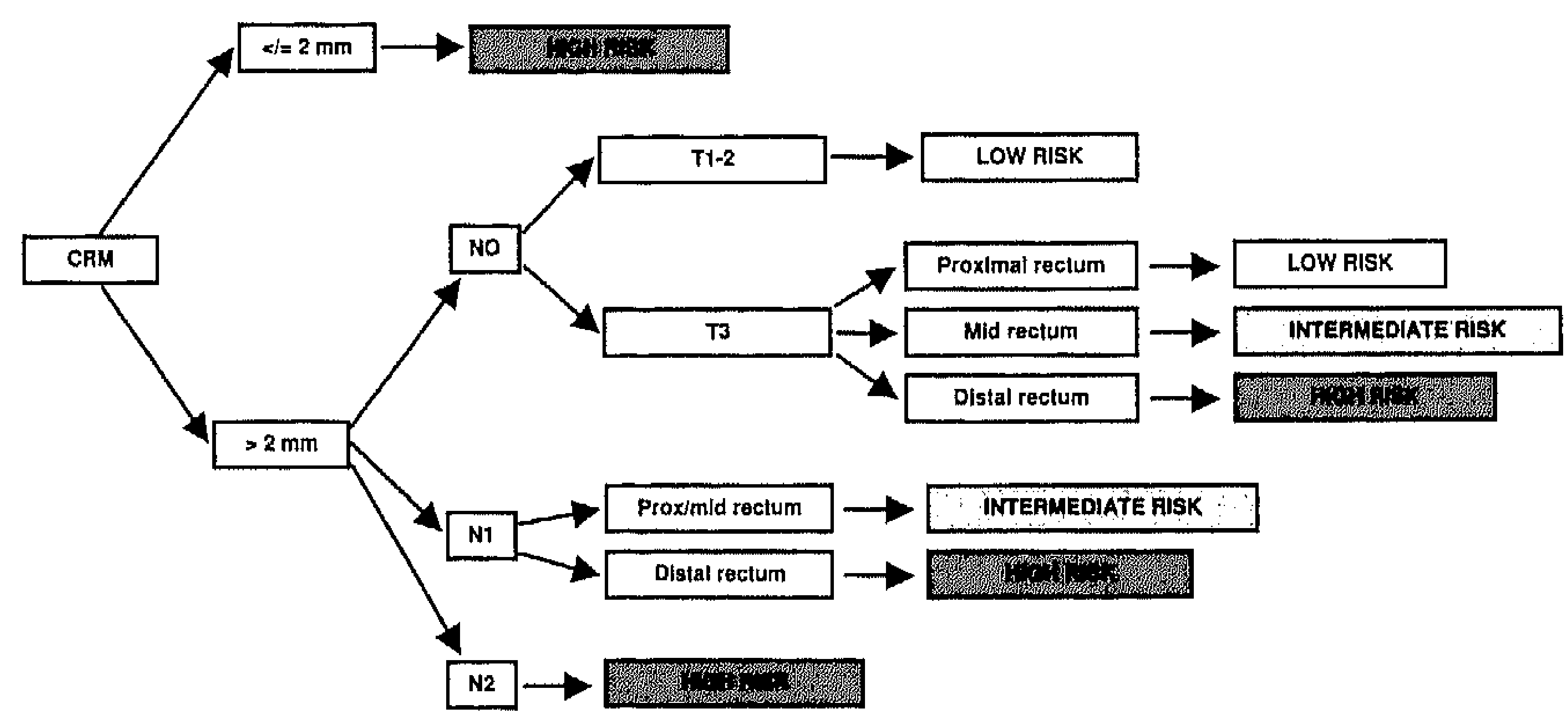

Figure 6.1 MRI-based decision scheme for tailored treatment of rectal cancer

\section{Neoadjuvant treatment}

Patients who received $5 \times 5$ Gy radiation therapy underwent a resection within one week. The long course of chemoradiation consisted of radiation therapy given in 28 fractions of $1.8 \mathrm{~Gy}$, on weekdays, and of chemotherapy, either oral capecitabine $\left(825 \mathrm{mg} / \mathrm{m}^{2}\right.$, twice a day for seven days a week), or capecitabine/oxaliplatin (oxaliplatin $130 \mathrm{mg} / \mathrm{m}^{2}$ on day one) in case of distant metastases. Patients underwent surgery 6-8 weeks after the last fraction of radiation therapy. One to two weeks before surgery patients underwent a new pelvic MRI with USPIO, to evaluate local status as a roadmap for surgery. Patients treated with combined chemoradiation therapy received a total of 6 courses of postoperative chemotherapy (capecitabine or oxaliplatin/capecitabine). 
Postoperative chemotherapy was also advised for patients who did not undergo long course chemoradiation therapy and who had positive lymph nodes at histology.

\section{Surgical treatment}

The standard surgery was a low anterior resection or abdominoperineal resection (depending on tumor height), according to TME-principle as described by Heald ${ }^{2}$. In selected cases of early tumors a TEM (transanal endoscopic microsurgery) was performed. For locally advanced tumors, the extent of the resection was based on the MR images. When required the TME resection was extended beyond the mesorectal fascia, an en-bloc resection of surrounding organs ('extended TME') was performed, or a posterior or total pelvic exenteration.

\section{Histological evaluation}

The resection specimen was evaluated in a standardized way, as described by Quirke $^{18}$ : (1) the specimen was fixed in formalin for $24-48 \mathrm{hrs}$, (2) the circumferential resection plane was inked, (3) the specimen was sliced transversely every $5 \mathrm{~mm}$, to determine the location of the smallest distance from tumor to resection plane and to search for lymph nodes. The pathologist recorded $T$ stage, circumferential resection margin (CRM), nodal status, distance of the tumor to proximal and distal resection margin, and completeness of the resection ( $R o / R 1$ ). A complete resection (Ro) was defined as a circumferential resection margin of $\geq 1 \mathrm{~mm}$. For the patients who underwent TEM, a complete resection of the tumor at histology as well as a confirmed No status at follow up MRI was considered a complete resection.

\section{Follow up}

Follow up after treatment for rectal cancer was done according to national guidelines. For those patients who underwent a TEM, follow-up MRI was performed at least after six months and one year, in addition to endoscopic follow up. 
Chapter 6

\section{Statistical analysis}

In this prospective cohort study the primary endpoint was the percentage of complete resections, as compared to a historical control, i.e. the data of the Dutch TME trial ${ }^{7}$. Comparison between groups was made using Chi-square test.

Power calculation of sample size: The percentage of complete resections in the Dutch TME trial was $84 \%^{7}$. An increase of this percentage to above $90 \%$ was considered to be clinically relevant. Data on prediction of circumferential resection margin on MRI suggest an accuracy of MRI over $90 \%$. Assuming an accuracy of $90 \%, 222$ patients were needed to reject with a power of $80 \%$ and $95 \%$ confidence the hypothesis that the proportion of Ro resections is $85 \%$ or lower.

Secondary endpoints were recurrence-free and overall survival. Recurrence-free and overall survival analyses were carried out by the Kaplan-Meier method. Log. rank test was used to compare survival in different subgroups. Event-free times were defined as the time from the day of surgery until the day of recurrence, death, or last follow-up.

All statistical analyses were performed using SPSS (version 12.0.1, 2003; SPSS, Chicago, IIl.).

\section{RESULTS}

Overall 296 patients were included in the prospective MRI-USPIO project, 83 in the single center pilot study and 213 in the multicenter study. Of these patients, 13 were not evaluable because of MRI-related problems (artifacts, refusal) $(n=5)$ and refusal of treatment $(n=8)$. Of the remaining 283 patients 5 had a previous malignancy, one a coexisting malignancy, and 47 were not treated with a curative intent because of widespread metastatic disease $(n=46)$ and because of very poor general condition $(n=1)$.

The present study population therefore consists of 230 patients who underwent treatment with curative intent, and were stratified into different treatment groups according to our study protocol. Sixteen of these patients presented with synchronous metastatic disease that was considered potentially curable. The male : female ratio of the patients was $134: 96$, mean age $( \pm S D)$ was $67( \pm 10.5)$ years. 
In 31 patients the actual treatment differed from the MRI based proposal. This was most often due to advanced age or poor condition of the patient, illustrated by 20 patients with a locally advanced tumor as classified by MRI treated with $5 \times 5$ Gy $(n=18)$ and surgery only $(n=2)$. In 7 patients surgery without radiotherapy was chosen whereas MRI advised short course radiotherapy and 4 patients received short course radiotherapy whereas MRI advised surgery only. Figure 6.2 shows the stratification of the actual received treatment.

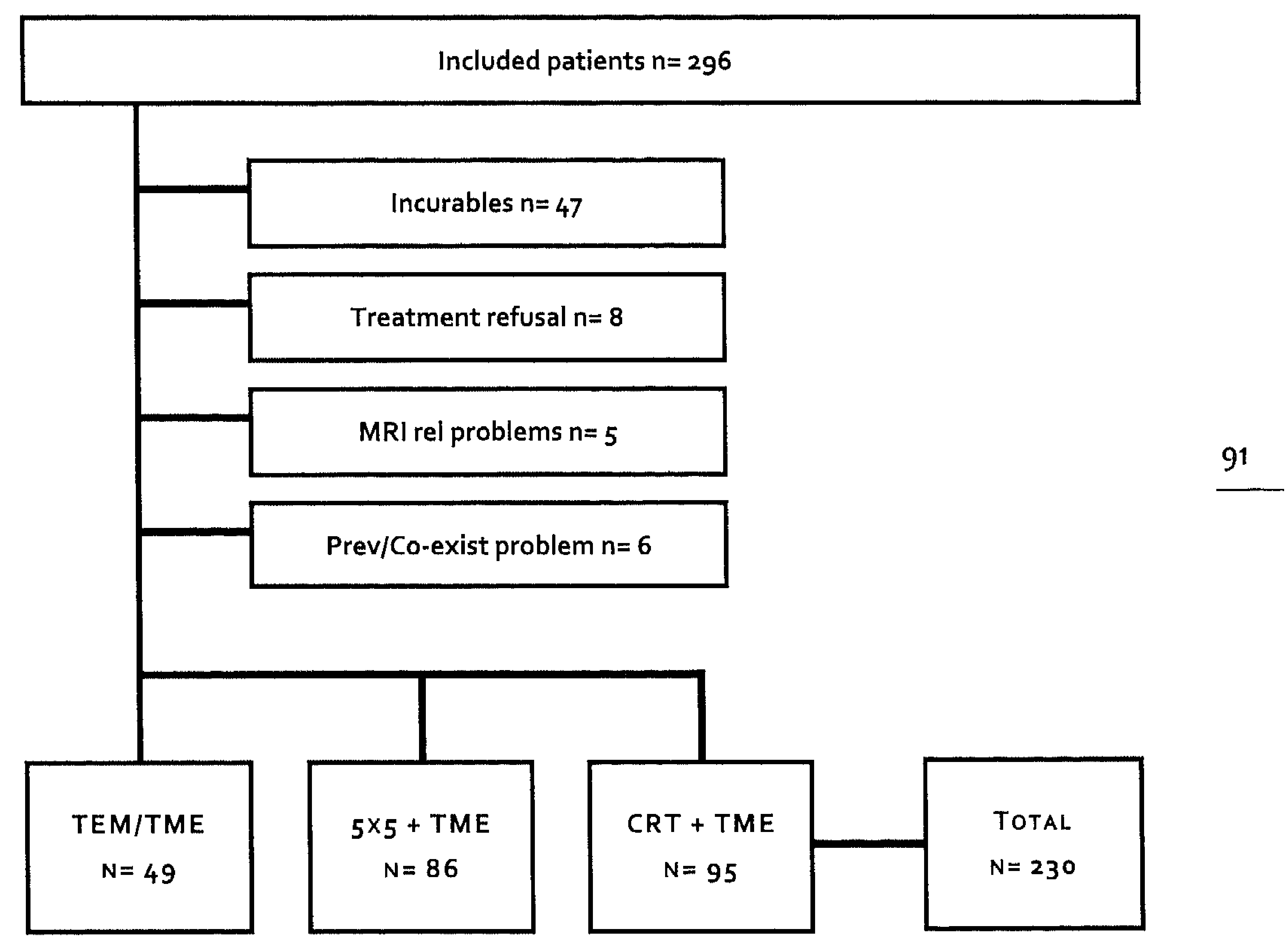

Figure 6.2 Stratification of patients based on the actual received treatment

The types of resections are shown in table 6.1. Two patients had a complete clinical response to chemoradiation, confirmed by repeated biopsies and imaging. These patients preferred not to undergo surgery and are currently in a follow up protocol. They were not included in the analysis for complete resections but were included in the follow up analysis. 
Chapter 6

\begin{tabular}{lc}
\hline & Total N (TME + extended TME) \\
\hline TEM & 21 \\
LAR w/ primary anastomosis & $114(103+11)$ \\
APR & $71(46+25)$ \\
Total pelvic exenteration & 1 \\
Posterior pelvic exenteration & 1 \\
Hartmann's procedure & $18(15+3)$ \\
Other & 2 \\
No resection & 2 \\
Total & 230 \\
\hline Table 6.1 Types of resection performed. Numbers in parentheses differentiate between the number of patients in \\
which the TME resection plane was followed and patients in which a wider resection was performed
\end{tabular}

\section{Complete resections}

The number of complete resections in the remaining 228 patients was 218 (95.6\%). The number of complete resections in the Dutch TME trial was 1514 out of 1805 eligible patients $(84 \%)^{7}$. Compared to the data of the Dutch TME trial, there is a statistically significant increase in the number of complete resections in the current study $(p<0.0001)$. See table 6.2 for the distribution of complete resections among the different treatment groups. Of the ten incomplete resections, 8 had an involved circumferential margin, and two an involved distal margin. In these 10 incomplete resections there were 5 tumor perforations ( $3 / 5$ abdominoperineal resection (APR)). There was however not a single case in which an underestimation of the circumferental resection margin was the cause of the incomplete resection. When there was an involved margin after surgery, this was either due to failure in surgical technique: tumor perforation during surgery or incomplete removal of the entire mesorectum, or surgical planning: insufficient attention to radiological evaluation of MR images after chemoradiation, that indicated that the margin was still at risk. Overall, half of the incomplete resections were due to problems in surgical planning and half of them were due to problems in surgical technique.

\section{Histology}

For distribution in the different stages at histopathological examination after surgery, see table 6.2. 
Tailored Treatment of Rectal Cancer

\begin{tabular}{lcccccc}
\hline & N Ro (\%) & 0 & I & II & III & IV \\
\hline TEM $(n=21)$ & $21(100)$ & 0 & 21 & 0 & 0 & 0 \\
TME $(n=28)$ & $28(100)$ & 0 & 13 & 6 & 9 & 0 \\
$5 \times 5$ Gy + TME $(n=86)$ & $82(95.3)$ & 0 & 29 & 28 & 23 & 6 \\
CRT + surgery $(n=93)^{1,2}$ & $87(93.5)$ & 14 & 22 & 23 & 24 & 10 \\
Total $(n=228)$ & $218(95.6)$ & 14 & 85 & 57 & 56 & 16 \\
\hline
\end{tabular}

Table 6.2 Number of complete resections and distribution of AJCC Stage at histology

${ }^{1}$ AJCC Stage for these patients is stage after neoadjuvant therapy, so ypTNM

${ }^{2} 2 / 95$ patients did refuse surgery after CRT and were therefore left out of the analysis of complete resections

\section{Perioperative period}

All TEM procedures $(n=21)$ were performed during a short hospital stay of $2-3$ days and were uncomplicated. For the other patients $(n=207)$, median hospital stay was 11 days (range: 4-199). The postoperative complications are listed in table 6.3. In 47 patients (20\%), there were major complications like anastomotic leakage, abscess needing surgical intervention, urinary leakage, respiratory insufficiency, ileus needing surgical intervention. In another 76 patients (33\%) there were less severe complications such as wound/urinary tract infections, uncomplicated pneumonia, conservatively managed ileus. Thirty-two patients required a surgical intervention for their complication, ranging in severity from drainage of a wound abcess $(n=8)$ to renewed laparotomy because of anastomotic leakage $(n=12)$. There were 31 patients who were readmitted to the hospital because of complications (within 30 days after initial surgery), 22 of them needed surgical intervention.

\begin{tabular}{lc}
\hline & N \\
\hline Wound dehiscence & 4 \\
Ileus & 21 \\
Anastomotic leakage & 21 \\
Urinary retention & 20 \\
Presacral abces & 12 \\
Wound infections & 28 \\
Urinary tract infections & 11 \\
Pneumonia & 13 \\
Sepsis & 13 \\
Pulmonary & 9 \\
Cardial & 17 \\
Other & 39 \\
\hline Table 6.3 Postoperative complications &
\end{tabular}




\section{Chapter 6}

Ten patients died postoperatively during their initial hospital stay, six of whom within 30 days. The postoperative mortality of all patients (including TEM) is therefore $4.3 \%$ with a 30 -day mortality of $2.6 \%$. This compares well with the $30-$ day mortality of $3 \%$ in the Dutch TME trial ${ }^{19}$. In 4 patients death was directly related to surgical complications, the other 6 patients died of (often multiple) systemic postoperative complications (e.g. myocardial infarction).

\section{Follow up}

The median follow up is 18.7 months (range: $4.3-63.6$ months). Three patients developed a local recurrence, resulting in a two year actuarial local recurrence rate of $2.8 \%$ (95\%-Cl 1.0-4.6\%) (figure 6.3).

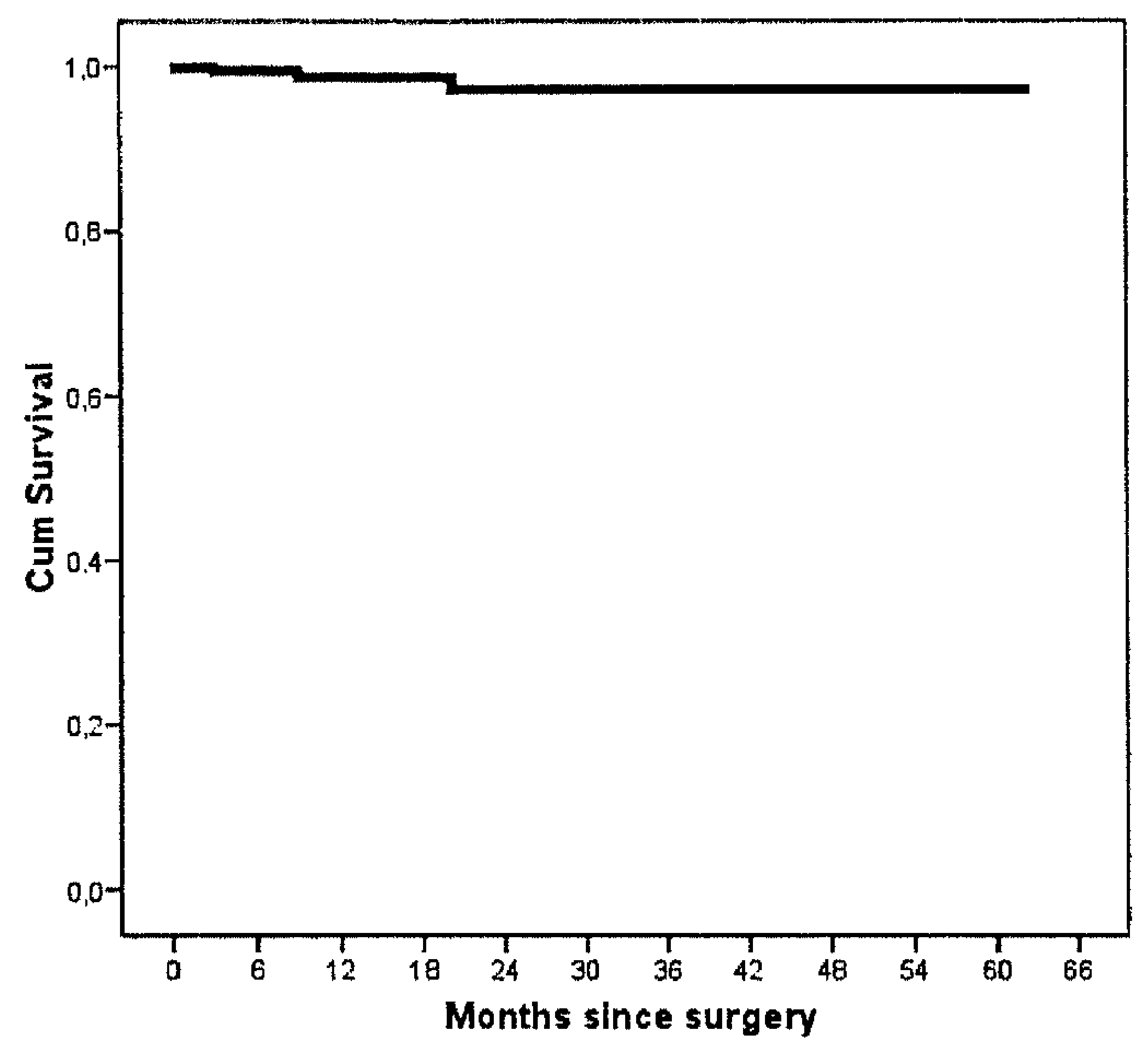

\begin{tabular}{lllllll}
$\begin{array}{l}\text { Months since } \\
\text { surgery }\end{array}$ & 0 & 12 & 24 & 36 & 48 & 60 \\
\hline Numbers at risk & 230 & 118 & 50 & 21 & 5 & 2
\end{tabular}

Figure 6.3 Local recurrence free survival including number at risk

One patient, who initially had a locally advanced tumor treated with CRT and Ro resection, developed a local recurrence as well as liver and lung metastases at 10 months follow up, and received palliative chemotherapy. The second patient was 
staged as a node negative tumor and underwent a TME. At histology there was one positive mesorectal lymph node and a narrow distal margin of $5 \mathrm{~mm}$. At 20 months an anastomotic recurrence was diagnosed and treated with CRT and resection. The third patient was staged as a superficial early tumor and underwent a TEM procedure. At histology the highly differentiated $T_{1}$ tumor was completely removed with a wide margin. At 3 months a follow up MRI revealed positive mesorectal lymph nodes, a sigmoidoscopy a new tumor just proximal to the scar, and a CT abdomen a liver metastasis. The patient underwent a TME after a short course of radiotherapy and currently receives chemotherapy for his liver metastasis.

Distant recurrence-free survival and overall survival is shown in figures 6.4 and 6.5 .

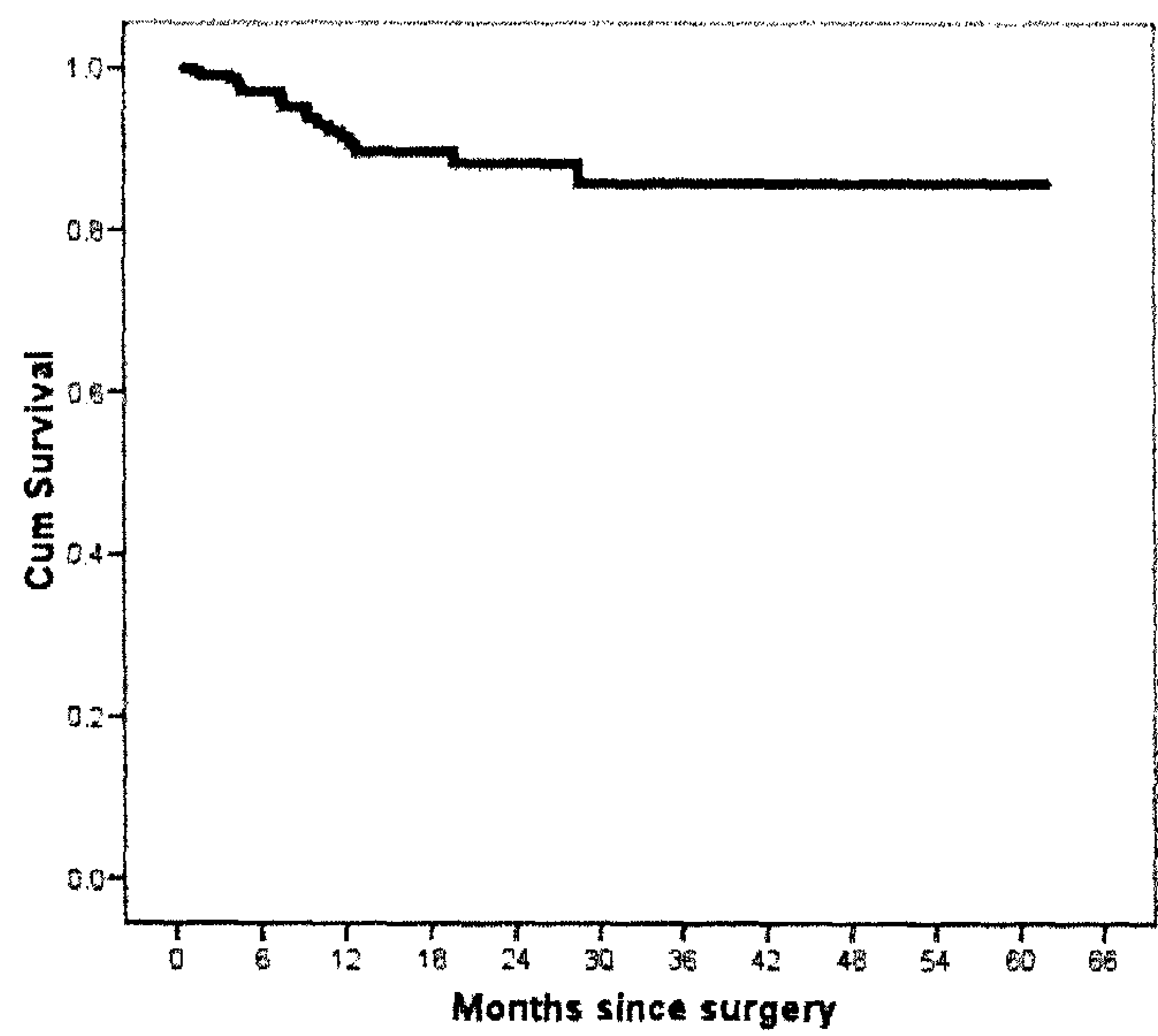

\begin{tabular}{lllllll}
$\begin{array}{l}\text { Months since } \\
\text { surgery }\end{array}$ & 0 & 12 & 24 & 36 & 48 & 60 \\
\hline Numbers at risk & 230 & 119 & 46 & 18 & 4 & 1
\end{tabular}

Figure 6.4a Distant recurrence free survival ind luding numbers at risk

The 2 year overall survival for the whole group is $86.5 \%$ (95\% Cl 83.3-89.7) The survival for the patients with non-locally advanced tumors is unexpectedly worse 


\section{Chapter 6}

than for patients with a locally advanced tumor $(p=0.015)$. This finding has been further explored. The steep drop at the beginning of the curve suggests an excess of postoperative mortality in this group.

\begin{tabular}{lccc}
\hline & Treatment & Cancer & Non-related \\
\hline TEM $(n=21)$ & 0 & 0 & 0 \\
TME $(n=28)$ & 1 & 0 & 1 \\
$5 \times 5$ Gy + TME $(n=86)$ & 6 & 4 & 7 \\
CRT + TME $(n=95)$ & 4 & 0 & 0 \\
Total $(n=230)$ & 11 & 4 & 8 \\
\hline Tabbe 6.4 Mortity (treatment- can
\end{tabular}

Table 6.4 Mortality (treatment-; cancer-; or non-related death)

The number of complications in the non-locally advanced group of patients is higher than in the locally advanced group ( 29 vs 18\%, $p=0.087$ ), and there is an excess of non-cancer-related mortality (7/86 vs $0 / 95 i p=0.005$ ) (see table 6.4).

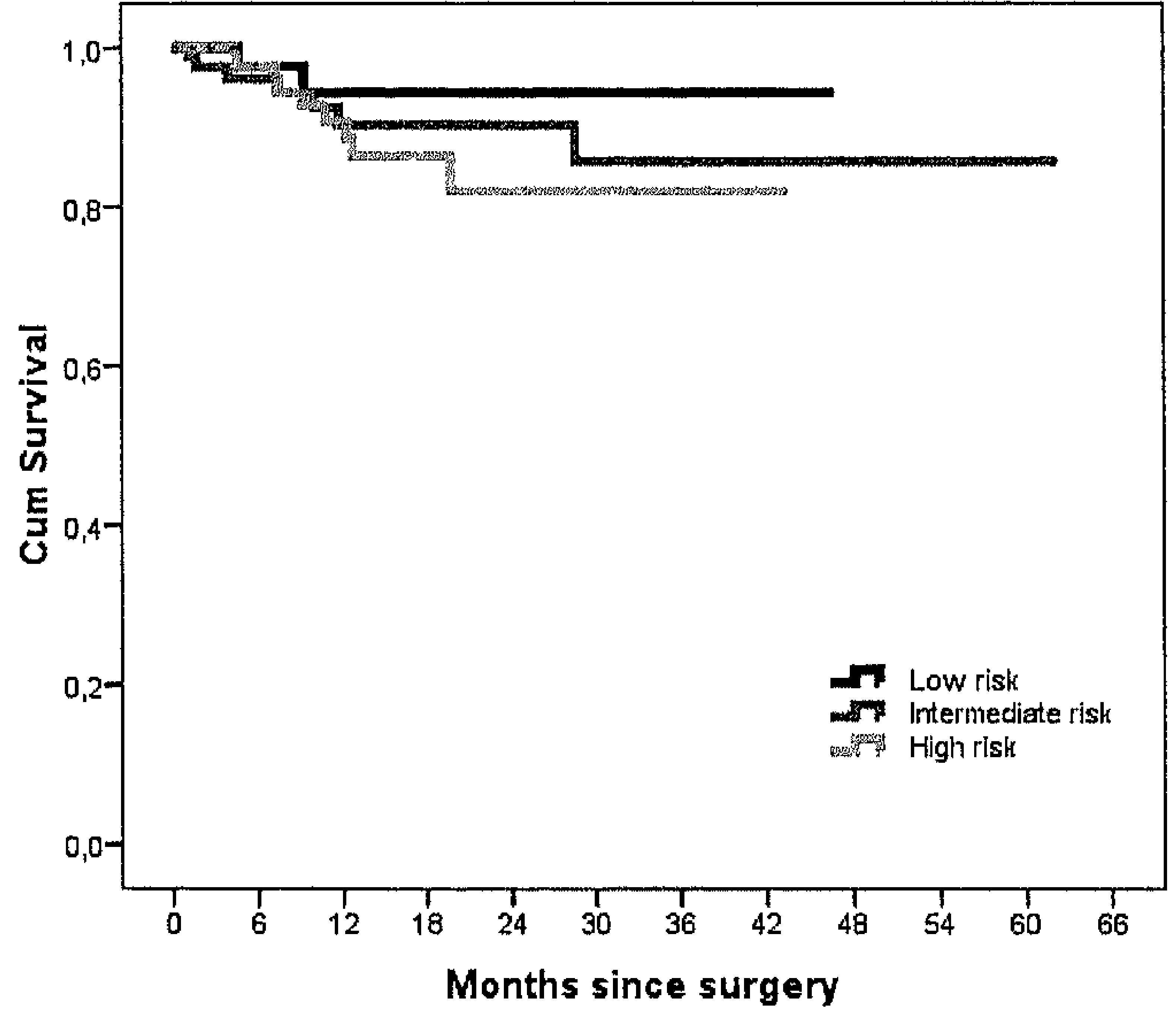

\begin{tabular}{lllllll}
$\begin{array}{l}\text { Months since } \\
\text { surgery }\end{array}$ & 0 & 12 & 24 & 36 & 48 & 60 \\
\hline $\begin{array}{llllll}\text { Numbers at risk } \\
\text { Low risk }\end{array}$ & 49 & 27 & 11 & 2 & 0 & 0 \\
Intermediate risk & 86 & 40 & 22 & 12 & 4 & 1 \\
High risk & 95 & 42 & 13 & 4 & 0 & 0
\end{tabular}


The patients in the $5 \times 5$ Gy group are also older than those in the group of locally advanced patients ( 68 vs 65 years, $p=0.062$ ).

\section{DISCUSSION}

This study shows that tailored treatment of primary rectal cancer based on preoperative MR imaging leads to an increased number of complete resections (95.6\%) as compared to a historical control (84\%), in which no standardized imaging was used ${ }^{7}$. Incomplete resection, as defined by a circumferential margin of $1 \mathrm{~mm}$ or less, is a strong predictor of local recurrence and survival ${ }^{20}$. The 2 -year local recurrence rate in this cohort is $2.8 \%$, and the overall survival $86.5 \%$. The low local recurrence rate at 2 year is encouraging, although a longer follow up is required to provide more proof for the value of our MR guided differentiated treatment approach.

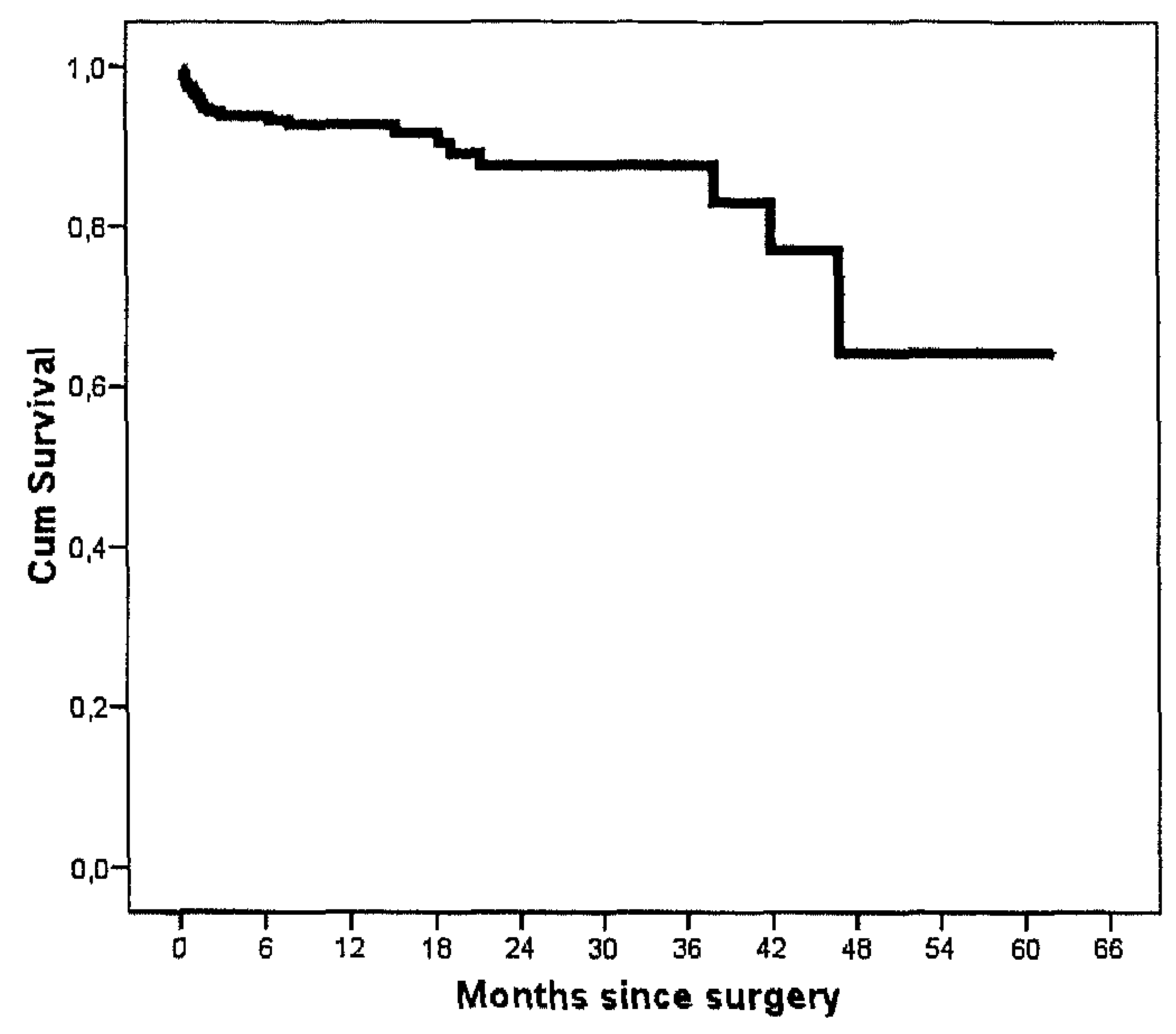

\begin{tabular}{lllllll}
$\begin{array}{l}\text { Months since } \\
\text { surgery }\end{array}$ & 0 & 12 & 24 & 36 & 48 & 60 \\
\hline Numbers at risk & 230 & 119 & 51 & 21 & 5 & 2
\end{tabular}

Figure 6.5a Overall survival including numbers at risk 


\section{Chapter 6}

The number of complete resections after TME surgery is rather constant in published series, with Ro rates of $84-91 \%{ }^{21},{ }^{22}$. Another study in which preoperative MR imaging was used to apply differentiated treatment showed an Ro rate of $87 \% \%^{23}$.

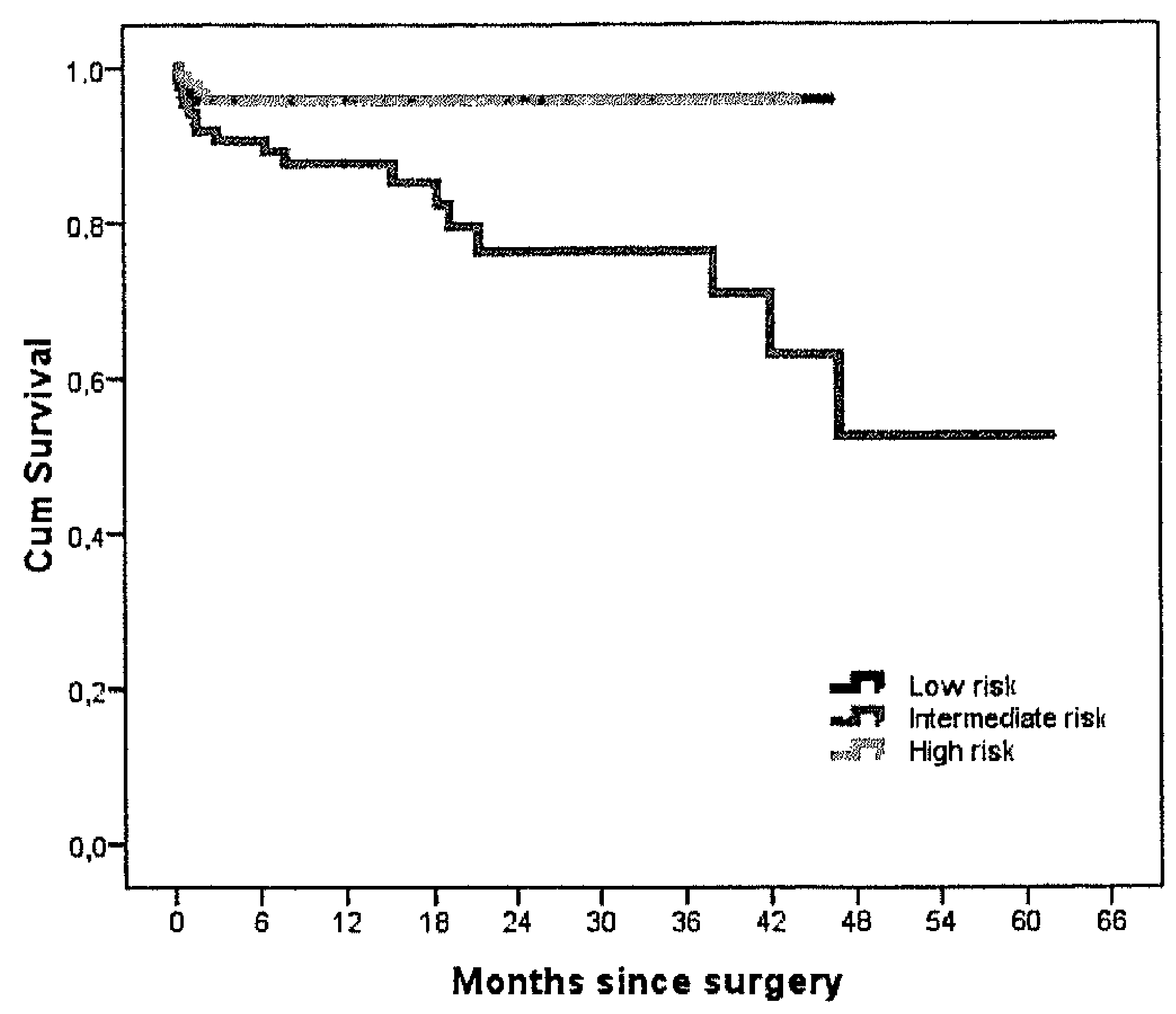

\begin{tabular}{lllllll}
$\begin{array}{l}\text { Months since } \\
\text { surgery }\end{array}$ & 0 & 12 & 24 & 36 & 48 & 60 \\
\hline Numbers at risk & 49 & 27 & 11 & 2 & 0 & 0 \\
Low risk & 86 & 45 & 24 & 14 & 5 & 2 \\
Intermediate risk & 85 & 47 & 16 & 5 & 0 & 0 \\
High risk & 95 & & & &
\end{tabular}

Figure 6.56 Overall survival for the different risk groups including numbers at risk

Our study with MRI-based differentiated treatment shows an Ro resection rate that is a little better than the above mentioned series. Several large prospective randomized trials have addressed the outcome after rectal cancer surgery, with a variety of (neo)adjuvant treatments regimens. Due to different definitions and inclusion criteria, comparing results can be misleading. Some studies tried to exclude stage I tumors ${ }^{3,4}, 6$, most studies excluded the truly locally advanced 'fixed' tumors, 21, 22, 24, and some studies have an upper age limit ${ }^{3,6}$. Our study included all consecutive primary rectal cancers, from the smallest superficial tumor to the largest tumor with invasion in surrounding structures, and should be 
considered as an 'all comers' study. Both the Dutch TME trial and the Swedish Rectal Cancer Trial (SRCT) studied the effect of $5 \times 5$ Gy preoperatively, either with (Dutch TME trial) or without (SRCT) standardized TME surgery, for 'nonclinically-fixed' tumors' ${ }^{72}$. The local recurrence rate in the $5 \times 5$ Gy arm of the Dutch TME trial is $5.6 \%$ at 5 years ${ }^{25}$ and in the SRCT $11 \%$ at 5 years ${ }^{24}$. In the study of Saver et al, a LR rate of $6 \%$ at 5 years was shown for locally advanced tumors ( $T_{3}$ or $\mathrm{N}_{1}$ ) treated with preoperative chemoradiation therapy ${ }^{3}$, Bosset et al showed a $L R$ rate of $7.6 \%$ for locally advanced tumors $\left(\mathrm{T}_{3} / 4\right.$ or $\left.\mathrm{N}_{1}\right)$ treated with preoperative chemoradiation and additional postoperative chemotherapy ${ }^{4}$. The $2.8 \%$ local recurrence rate at 2 years compares favorably with the above studies, although a longer follow up is required for more definitive results. It is our belief that it is the combination of good preoperative imaging, good multidisciplinary preoperative planning and execution of the chosen treatment that leads to a good outcome.

\section{Tailored treatment}

Although our data provide no proof that individualization of treatment in rectal cancer is better than applying a single standard treatment for all patients, the differentiated treatment is expected to lead to an optimal balance between minimizing treatment related morbidity and best oncological outcome. With our very high number of complete resections and the low local recurrence rates we achieved a good overall oncological outcome. In patients with truly locally advanced disease, with tumor growth beyond the mesorectal fascia, a standard resection results in a high percentage of positive margins. These tumors are well identified with $\mathrm{MRI}^{8}$, and treated with neoadjuvant chemoradiation. The downsizing effect of chemoradiation increases the chances of a complete resection ${ }^{6}$. In our study this group constitutes $41 \%$ of patients, indicating a liberal use of the term locally advanced disease. Apart from tumor growth into the mesorectal fascia, or within $1 \mathrm{~mm}$, we also considered $\mathrm{N}_{2}$ disease as locally advanced. Furthermore, in distal tumors the anticipated margin is often narrow, and as a subgroup analysis of the data of the Dutch TME trial suggests that a short course of preoperative radiation is insufficient to prevent local recurrences ${ }^{26}$, these were often also included in the chemoradiation group. Another $37 \%$ of patients were treated with $5 \times 5$ and TME, a schedule that has 


\section{Chapter 6}

proven to be effective both in the SRCT and the Dutch TME trial. In a Polish randomized trial of patients with resectable T3-4 tumors the short course has been shown to be as effective in both local control and overall outcome as chemoradiation, with less acute toxicity ${ }^{27}$. From a cost-efficiency point of view the short course is preferable to a long course of chemoradiation in patients in whom downsizing is not required. At the other end of the spectrum are patients with a very low risk for recurrence, patients with stage I disease, and patients with node negative tumors with a wide resection margin. All preoperative radiation schedules are associated with a worse functional long term outcome as compared to surgery alone $e^{29,27,28}$. Ideally these patients should be identified and treated with surgery without any form of neoadjuvant therapy, as this provides excellent oncological control with less morbidity, and less costs.

\section{Local excisions}

Local excision with the TEM technique is associated with less postoperative and functional morbidity as compared to $\mathrm{TME}^{29}{ }^{30}$. The use in early rectal cancer is controversial, and only small $\mathrm{T}_{1}$ tumors with favourable histological features are presently an accepted indication. T2 tumors have shown too high a local recurrence rate of $20 \% \%^{31}$. The majority of the recurrences are thought to arise from unrecognized nodal disease ${ }^{32}$. Because MRI with USPIO had previously shown a good sensitivity ${ }^{15}$, confirmed in our pilot study ${ }^{16}$, local excision with TEM was considered a good option in many patients with small tumors. Eventually $9 \%$ of the patients were treated with a TEM. They are in a follow up schedule that includes MRI, with at present only I local recurrence. Although this requires further studying, better lymph node staging seems to enable a good selection for a TEM procedure. A more controversial approach is the use of local excision or even a wait-and-see policy (only two patients in the present study) after a good response to chemoradiation ${ }^{33}, 34$. When considering a local excision after chemoradiation again accurate lymph node staging will be very helpful, and this requires further exploration ${ }^{35}$.

MRI as a selection tool

MRI has repeatedly been shown to provide accurate information on the prediction of involvement of the mesorectal fascia, the anticipated CRM in a 
standard resection ${ }^{8,23}$. Although we did not use it in our study, the depth of invasion beyond the rectal wall on MRI with a cut off of $5 \mathrm{~mm}$, has also been reported as a reproducible prognostic variable ${ }^{36}$. In our study we additionally tried to identify low risk patients, patients with a node negative small tumor, who have a negligible risk for local recurrence. Because the accuracy of lymph node staging with EUS and MRI with traditional size criteria yields accuracy figures of about only $70 \%{ }^{14}$, we used a lymph node-specific contrast agent (USPIO) to improve lymph node prediction. Overall, better accuracy was obtained, with a high sensitivity $(90 \%)$, but with less specificity $(53 \%)^{37}$. This was partly the result of the notion that overstaging and overtreatment result in less harm for patients than understaging and undertreatment, that led to cautious interpreting by the radiologists. The same cautious reporting also resulted in overstaging when the distinction between $T_{2}$ and $T_{3}$ was difficult. As a consequence many stage I patients were 'overtreated' with neoadjuvant therapy.

The radiological analysis of this multicenter project (see chapter 5 ) shows a trend towards a worse performance of 1.0Tesla MRI as compared to 1.5Tesla MRI for prediction of $\mathrm{T}$ and $\mathrm{N}$ stage. We would now therefore advise caution to use $1.0 \mathrm{~T}$ MRI for the assessment of these risk factors. In the present study, were the MR assessment of the CRM was given the most weight, the use of 1.0 Tesla did not negatively influence the outcome parameter of incomplete resections, as no single incomplete resection was due to faulty information provided by MR images. In situations where more weight is given to the nodal prediction, such as a local excision, the inferior performance of 1.0 Tesla becomes a critical issue.

As important as the quality of the MRI and the reporting is a good functioning preoperative multidisciplinary planning ${ }^{38,} 39$. Although the number of complete resections in the present series is high, it could even have been higher. Half of the incomplete resections were related to errors in surgical planning, in which not all of the available information was used. This was mostly in patients with locally advanced tumors where the MR images after chemoradiation can be difficult to interpret.

An unexpected finding in our study was the better survival of the group of patients with locally advanced cancer treated with chemoradiation as compared to the group of patients who received a short course of preoperative therapy. 
Chapter 6

Most likely this is the result of a selection bias, as the treatment protocol allowed the clinicians to deviate from the protocol when this was considered better for the patient. Old patients and patients with severe comorbidity were often treated with the less toxic short course instead of chemoradiation. In this group of patients there was more treatment related, and non cancer related mortality than in the group who received chemoradiation.

Limitations of the study

The main limitation of this study is the concept of a cohort study with a historical control. As the concept of a differentiated approach is gaining ground, a randomized trial comparing the differentiated approach with a uniform approach will be very difficult to perform. The 'historical' control in our study was the Dutch TME trial, a trial in which the accrual was completed only 3 years before the start of our study. The main difference is the incorporation of MRI in the present study, whereas imaging was not standard in the Dutch TME trial. It is therefore reasonable to assume that at least part of the improvement in complete resections is due to the implementation of preoperative imaging and its consequences.

Another limitation is the practical difficulty with MR lymph node staging using USPIO: although promising results with USPIO are described by Will et al. ${ }^{15}$ as well as by our own study group ${ }^{16}$, USPIO is still not available on the market.

\section{Conclusion}

Tailored treatment of rectal cancer based on dedicated preoperative MR imaging has increased the number of microscopically complete resections significantly as compared to a historical control. The advantage of tailored tre atment consists of the avoidance of over- and undertreatment in patients with different risk for local recurrence. The two year local recurrence rate of $2.8 \%$ in this group of patients is encouraging. A longer follow up is required to confirm whether the improvement in complete resections will indeed lead to better clinical outcome. 


\section{REFERENCES}

1. Heald RJ, Ryall RD. Recurrence and survival after total mesorectal excision for rectal cancer. Lancet 1986;1(8496): 1479-1.482.

2. Heald RJ. A new approach to rectal cancer, Br J Hosp Med 1979;22(3): 277-281.

3. Sauer R, Becker H, Hohenberger W, Rodel C, Wittekind C, Fietkau R, Martus P, Tschmelitsch J, Hager E, Hess CF, Karstens JH, Liersch T, Schmidberger $H$, Raab R. Preoperative versus postoperative chemoradiotherapy for rectal cancer. N Engl J Med 2004;351(17): 1731-1740.

4. Bosset JF, Collette L, Calais G, Mineur L, Maingon P, Radosevic-Jelic L, Daban A, Bardet E, Beny A, Ollier JC. Chemotherapy with preoperative radiotherapy in rectal cancer. $N$ Engl $J$ Med 2006;355(11): $1114-1123$.

5. Braendengen $M$, Tveit KM, Berglund A, Birkemeyer $E$, Frykholm G, Pahlman L, Wiig JN, Bystrom $P$, Bujko K, Glimelius B. Randomized phase III study comparing preoperative radiotherapy with chemoradiotherapy in nonresectable rectal cancer. J Clin Oncol 2008;26(22):3687-3694.

6. Gerard JP, Conroy T, Bonnetain F, Bouche $O$, Chapet $O$, Closon-Dejardin MT, Untereiner M, Leduc B, Francois E, Maurel J, Seitz JF, Buecher B, Mackiewicz R, Ducreux M, Bedenne L. Preoperative radiotherapy with or without concurrent fluorouracil and leucovorin in $T_{3}-4$ rectal cancers: results of FFCD 9203. J Clin Oncol 2006;24(28): 4620-4625.

7. Kapiteijn E, Marijnen CA, Nagtegaal ID, Putter $H$, Steup WH, Wiggers T, Rutten HJ, Pahlman L, Glimelius B, van Krieken JH, Leer JW, van de Velde CJ. Preoperative radiotherapy combined with total mesorectal excision for resectable rectal cancer. N Engl J Med 2001;345(9): 638-646.

8. Beets-Tan RG, Beets GL. Rectal cancer: review with emphasis on MR imaging. Radiology 2004;232(2): 335-346.

9. Beets-Tan RG, Beets GL, Vliegen RF, Kessels AG, Van Boven $H$, De Bruine A, von Meyenfeldt MF, Baeten $C G$, van Engelshoven JM. Accuracy of magnetic resonance imaging in prediction of tumourfree resection margin in rectal cancer surgery. Lancet 2001;357(9255): 497-504.

10. Brown G, Richards CJ, Newcombe RG, Dallimore NS, Radcliffe AG, Carey DP, Bourne MW, Williams GT. Rectal Carcinoma: Thin-Section MR Imaging for Staging in 28 Patients. Radiology 1999;211(1): 215-222.

22. Bissett IP, Fernando CC, Hough DM, Cowan BR, Chau KY, Young AA, Parry BR, Hill GL. Identification of the fascia propria by magnetic resonance imaging and its relevance to preoperative assessment of rectal cancer. Dis Colon Rectum 2001;44(2): 259-265.

12. Blomqvist $L$, Rubio $C$, Holm T, Machado $M$, Hindmarsh T. Rectal adenocarcinoma: assessment of tumour involvement of the lateral resection margin by MRI of resected specimen. $B r J$ Radiol 1999;72(853): 18:23.

13. Peschaud F, Cuenod CA, Benoist S, Julie $C$, Beauchet A, Siauve N, Taieb-Kasbi F, Penna $C_{1}$ Nordlinger $B$. Accuracy of magnetic resonance imaging in rectal cancer depends on location of the tumor. Dis Colon Rectum 2005;48(8): 1603-1609.

14. Lahaye $M J$, Engelen SM, Nelemans PJ, Beets GL, van de Velde CJ, van Engelshoven IM, Beets-Tan RG. Imaging for predicting the risk factors--the circumferential resection margin and nodal disease-of local recurrence in rectal cancer: a meta-analysis. Semin Uitrasound CT MR 2005;26(4): 259-268.

15. Will O, Purkayastha $S$, Chan C, Athanasiou T, Darzi AW, Gedroyc W, Tekkis PP. Diagnostic precision of nanoparticle-enhanced MRI for lymph-node metastases: a meta-analysis. Lancet Oncol 2006;7(1): $52-60$.

16. Lahaye MJ, Engelen SM, Beets GL, de Bruine AP, von Meyenfeldt MF, Van Engelshoven JM, Van de Velde Cl, Beets-Tan RG. How accurate can we predict the nodal status in primary rectal cancer with USPIO MRI? Ann Oncol 2006;17 (suppl): i11-12. 


\section{Chapter 6}

17. Nagtegaal ID, Marijnen CA, Kranenbarg EK, van de Velde CJ, van Krieken JH. Circumferential margin involvement is still an important predictor of local recurrence in rectal carcinoma: not one millimeter but two millimeters is the limit. Am J Surg Pathol 2002;26(3): 350-357.

18. Quirke P, Durdey $P$, Dixon MF, Williams NS. Local recurrence of rectal adenocarcinoma due to inadequate surgical resection. Histopathological study of lateral tumour spread and surgical excision. Lancet 1986;2(8514): 996-999.

19. Marijnen CA, Kapiteijn E, van de Velde CJ, Martijn H, Steup WH, Wiggers T, Kranenbarg EK, Leer JW. Acute side effects and complications after short-term preoperative radiotherapy combined with total mesorectal excision in primary rectal cancer: report of a multicenter randomized trial. $J$ Clin Oncol 2002;20(3): 817-825.

20. Nagtegaal ID, Quirke $P$. What is the role for the circumferential margin in the modern treatment of rectal cancer? J Clin Oncol 2008;26(2): 303-312.

21. Law WL, Chu KW. Anterior resection for rectal cancer with mesorectal excision: a prospective evaluation of 622 patients. Ann Surg 2004;240(2): 260-268.

22. Hall NR, Finan PJ, al-Jaberi $T$, Tsang CS, Brown SR, Dixon MF, Quirke P. Circumferential margin involvement after mesorectal excision of rectal cancer with curative intent. Predictor of survival but not local recurrence? Dis Colon Rectum 1998;41(8): 979-983.

23. Salerno G, Daniels IR, Moran BJ, Wotherspoon A, Brown G. Clarifying margins in the multidisciplinary management of rectal cancer: the MERCURY experience. Clin Radiol 2006;61(11): 916-923.

24. Improved survival with preoperative radiotherapy in resectable rectal cancer. Swedish Rectal Cancer Trial. N Engl J Med 1997;336(14): 980-987.

25. Peeters KC, Marijnen CA, Nagtegaal ID, Kranenbarg EK, Putter $H$, Wiggers $T$, Rutten $H$, Pahlman $L$, Glimelius $B$, Leer JW, van de Velde CJ. The TME trial after a median follow-up of 6 years: increased

26. Nagtegaal ID, van de Velde CJ, Marijnen CA, van Krieken JH, Quirke P. Low rectal cancer: a call for a change of approach in abdominoperineal resection. J Clin Oncol 2005;23(36): 9257-9264.

27. Bujko $K$, Nowacki MP, Nasierowska-Guttmejer $A$, Michalski $W$, Bebenek $M$, Pudelko $M$, Kryj $M$, Oledzki J, Szmeja J, Sluszniak J, Serkies K, Kladny J, Pamucka M, Kukolowicz P. Sphincter preservation following preoperative radiotherapy for rectal cancer: report of a randomised trial comparing short-term radiotherapy vs. conventionally fractionated radiochemotherapy. Radiother Oncol 2004;72(1): 15-24.

28. Peeters KC, van de Velde CJ, Leer JW, Martijn $H$, Junggeburt JM, Kranenbarg EK, Steup WH, Wiggers $T$, Rutten HJ, Marijnen CA. Late side effects of short-course preoperative radiotherapy combined with total mesorectal excision for rectal cancer: increased bowel dysfunction in irradiated patients--a Dutch colorectal cancer group study. J Clin Oncol 2005;23(25): 6199-6206.

29. Suppiah A, Maslekar S, Alabi A, Hartley JE, Monson JR. Transanal endoscopic microsurgery in early rectal cancer: time for a trial? Colorectal Dis 2008;10(4): 314-327; discussion 327-319.

3o. Borschitz T, Gockel I, Kiesslich R, Junginger T. Oncological outcome after local excision of rectal carcinomas. Ann Surg Oncol 2008;15(11): 3101-3108.

31. Whitehouse PA, Armitage JN, Tilney HS, Simson JN. Transanal endoscopic microsurgery: local recurrence rate following resection of rectal cancer. Colorectal Dis 2008;10(2): 187-193.

32. Whiteford MH. Transanal endoscopic microsurgery (TEM) resection of rectal tumors. $J$ Gastrointest Surg 2007i11(2): 155-157.

33. Habr-Gama A, Perez RO, Proscurshim I, Campos FG, Nadalin W, Kiss D, Gama-Rodrigues J. Patterns of failure and survival for nonoperative treatment of stage co distal rectal cancer following 


\section{Tailored Treatment of Rectal Cancer}

neoadjuvant chemoradiation therapy. J Gastrointest Surg 2006;10(10): 1319-1328; discussion 13281319 .

34. Borschitz $T$, Wachtlin $D$, Mohler $M$, Schmidberger $H$, Junginger T. Neoadjuvant chemoradiation and local excision for T2-3 rectal cancer. Ann Surg Oncol 2008;15(3): 712-720.

35. Beets GL, Lahaye MJ, Engelen SM, Kessels AG, Keymeulen K, De Bruine A, Willig AP, Van Engelshoven JM, Van de Velde CJ, Beets-Tan RG. USPIO MRI in rectal cancer: accuracy in primary staging and downstaging after chemoradiation, a multicenter study. . Ann Oncol 2008;19(suppl 1): ii1.

36. Brown $G$, Radcliffe $A G$, Newcombe RG, Dallimore NS, Bourne MW, Williams GT. Preoperative assessment of prognostic factors in rectal cancer using high-resolution magnetic resonance imaging. BrJSurg 2003;90(3): 355-364.

37. Lahaye MJ, Engelen SM, Beets GL, Kessels AG, Post PJ, Dohmen JP, Opdenakker GR, Van de Velde CJ, Van Engelshoven JM, Beets-Tan RG. Accuracy of USPIO-enhanced MRI for local staging of rectal cancer: a Multicenter Study in expert and three regional centers. . Ann Oncol 2008;19 (suppl 1): i33.

38. Valentini V, Glimelius B, Minsky BD, Van Cutsem E, Bartelink $H$, Beets-Tan RG, Gerard JP, Kosmidis $P$, Pahlman L, Picciocchi A, Quirke P, Tepper J, Tonato $M$, Van de Velde $C J$, Cellini N, Latini P. The multidisciplinary rectal cancer treatment: main convergences, controversial aspects and investigational areas which support the need for an European Consensus. Radiother Oncol 2005;76(3): 241-250.

39. Wiggers $\mathrm{T}$, van de Velde $\mathrm{CJ}$. The circumferential margin in rectal cancer. Recommendations based on the Dutch Total Mesorectal Excision Study. Eur J Cancer 2002;38(7): 973-976. 
Aggressive multidisciplinary treatment of locally advanced or unresectable rectal cancer. Local control is good, but patients still die of metastases

S.M.E. Engelen, E.A. Kouwenhoven, M. . Lahaye, R.G.H. Beets-Tan, G. Lammering, R.L.H. Jansen, K.B.M.I. Keymeulen, C.H.C. Dejong, G.L. Beets 


\section{Chapter 7}

\section{ABSTRACT}

Aim A multidisciplinary approach is important in the challenging treatment locally advanced rectal or unresectable cancer. The aim of this study was assess the current results of an aggressive multimodality treatment of the patients in a tertiary referral center.

Methods Patients with primary locally advanced or unresectable rectal canc who underwent multimodality treatment (including neoadjuva (chemo)radiotherapy, surgical resection, brachytherapy and adjuva chemotherapy) in a University tertiary referral center between January 1998 ar December 2006 were analysed. Parameters studied were proportion of comple resections, postoperative complications, hospital stay, recurrence-free surviv and overall survival.

Results 117 Patients were enrolled in this study. There were 91 resections wit curative and 26 with palliative intention. There were significantly more complet resections in the resections with curative intention (96\%) as compared to th palliative resections $(62 \%) \quad(p=0.000)$. Thirty-five patients had majs postoperative complications. Thirty-day mortality rate was $2.6 \%$. Five-yei follow-up revealed a local control in $90 \%$ of patients and an overall survival rate $65 \%$.

Conclusion This study demonstrates that excellent local control can be obtaine in locally advanced primary rectal cancer with the use of aggressiv multimodality treatment. This is achieved at the expense of considerabl morbidity. Unfortunately many patients still die of metastatic disease, and futur research should be focused on systemic treatment regimens for better distar control. 


\section{INTRODUCTION}

The treatment of locally advanced or unresectable rectal cancer can be challenging. There is at present no consensus on the precise definition of what constitutes a locally advanced or unresectable rectal cancer. We have used the definition of any tumor that has spread into or beyond the mesorectal fascia. These patients not only face the threat of a high risk for distant metastases, but also the risk of a local recurrence, with often debilitating symptoms. The treatment goal is therefore not only cure, but also optimal local control. This requires a multidisciplinary approach.

The first step is the identification of the locally advanced nature of the disease. In the past surgeons found themselves all too often confronted during the operation with an unexpected large tumor growing into surrounding organs. It has now become clear that imaging, preferably with MR, is able to identify tumors that have breached the mesorectal fascia ${ }^{1}$.

The second step is neoadjuvant chemoradiation, to downsize the tumor in order to obtain more complete resections. The most commonly used regimen is a $5 \mathrm{FU}$ based 6 week course combined with 45-50.4 Gy, but many centers are presently evaluating more effective, and often more toxic regimens ${ }^{2-4}$.

The third step is the surgical resection, with an en-bloc resection of all involved or adherent organs and structures. The aim should be a histologically verified tumor free margin of at least $1 \mathrm{~mm}^{5}$. It remains very difficult to predict on imaging whether or not there is viable tumor present in the radiotherapy induced fibrosis. Therefore the resection plan is usually made based on the initial MR images, with resection of the whole area of the initial tumor presentation. In some areas, like the lateral pelvic wall or the sacral bone above $\mathrm{S}_{3}$, a wide tumor free resection margin may be difficult to obtain. Equally this may only be achieved at the expense of major morbidity ${ }^{6,7}$. Intra-operative radiotherapy or intra- or postoperative brachytherapy can be used to achieve optimal local control. Although no randomized trials have been performed to prove the usefulness of this approach, it is advocated by many expert centers for the most difficult of cases $^{8-11}$.

The last step in the treatment is adjuvant chemotherapy to cure micro-metastatic disease. Although the evidence in rectal cancer is not as solid as for colonic 


\section{Chapter 7}

cancer, many rectal cancer patients receive adjuvant chemotherapy ${ }^{12}$. Locally advanced rectal cancer patients, with their high risk for distant metastases, will probably benefit even more from effective systemic therapy ${ }^{13}$.

The aim of the present study was to assess the results of an aggressive multimodality approach for the treatment of patients with locally advanced rectal cancer. The identification of strengths and weaknesses in the current approach is required for further improvement of the treatment of this challenging disease.

\section{METHODS}

All patients with histologically proven primary locally advanced rectal cancer who underwent surgical treatment in Maastricht University Medical Center from January 1998 until December 2006 were analysed. The University Hospital serves as a tertiary referral center for 5 regional district general teaching hospitals, covering a population of 860.000 with a average incidence of 115 new rectal cancer cases per year ${ }^{14}$. 'Locally advanced' tumors were defined as tumors in which, based on imaging, a standard TME resection would not result in a clear margin: $T_{3}$ tumors reaching $<2 \mathrm{~mm}$ from or infiltrating the mesorectal fascia or $\mathrm{T}_{4}$ tumors invading adjacent pelvic structures. Imaging was performed with magnetic resonance $(n=101)$, or in the early study period by CT scan $(n=16)$. All patients were screened for distant metastases with an abdominal and chest $C T$ scan (or chest X-ray in the early study period).

Neoadjuvant therapy was considered in the most advanced cases in the nineties, and became standard for all patients with locally advanced cancer in 2000. Neoadjuvant therapy consisted mostly of a long course of radiotherapy (45-50.4 Gray, 1.8Gray/day) or chemoradiotherapy (45-50.4 Gray, 1.8Gray/day + 5FU/Leucovorin or Capecitabine as radiosensitizer). A second MRI was performed 4-6 weeks after the last dose of (chemo) radiation to assess tumor response. Generally the plan for the surgical resection was made on the initial imaging before neoadjuvant therapy, and all involved structures were resected en bloc with the aim to obtain a microscopically complete (Ro) resection. In palliative resections, or in a few patients with severe comorbidity and a good response to chemoradiation, it was decided to perform a less extensive resection, and to accept the slightly higher risk of a close or involved margin. The policy for 
structures at risk for invasion was to resect en bloc rather than to apply brachytherapy. Catheters for postoperative brachytherapy were only implanted when resection of the structures at risk was not possible or associated with a high risk of complications, such as the sacral bone above S2. Postoperative brachytherapy was applied as PDR (pulse dose rate), $25 \mathrm{~Gy}$ in pulses of $0.5 \mathrm{~Gy} / \mathrm{hr}$ in the early study period and as HDR (high dose rate), fractionated as $8 \times 3 \mathrm{~Gy}$, twice daily in the later study period (as of July 1999).

Before 2003, 5-FU based adjuvant chemotherapy was administered in case of a ypT 4 and/or ypN+tumor. As from 2003, all patients with a locally advanced rectal cancer were considered at risk for distant metastases and were offered postoperative chemotherapy, irrespective of ypT and ypN stage.

Histopathologic evaluation of all resection specimens was standardized, as described by Quirke ${ }^{15}$. (1) the specimen was fixed in formalin for $24-48 \mathrm{hrs},(2)$ the circumferential resection plane was inked, and finally (3) the specimen was sliced transversely every $5 \mathrm{~mm}$, to determine the location of the shortest distance from tumor to resection plane and to search for lymph nodes. The pathologist evaluated $T$ stage, the circumferential resection margin (CRM), nodal status, distance of the tumor to proximal and distal resection margin, as well as completeness of the resection $\left(R_{0} / R_{1} ; R o=C R M>1 \mathrm{~mm}\right.$ and free proximal/distal margin).

Parameters studied were co-morbidity, proportion of complete resections, postoperative complications, hospital stay, recurrence-free survival and overall survival.

Statistical analysis was performed using the Pearson Chi-square test to compare groups for categorical variables; a $p<0.05$ was defined as being significant. Recurrence-free and overall survival analyses were carried out by the KaplanMeier method. Event-free times were defined as the time from the day of surgery until the day of recurrence, death, or last follow-up. All statistical analyses were performed using SPSS (version 12.0.1, 2003i SPSS, Chicago, III.). 


\section{Chapter 7}

\section{RESULTS}

There were 117 patients (66 men and 51 women) with histologically proven primary locally advanced rectal cancer who underwent rectal surgery between January 1998 and December 2006. Of these patients, 64 (55\%) were referred from other hospitals. Ninety-one patients $(78 \%)$, of whom 5 had synchronous distant metastases, underwent a resection with curative intent. Twenty-five patients underwent a palliative resection because they had wide-spread metastatic disease at the time of surgery and one patient because of inoperable local disease.

The median age of the patients at diagnosis was 65.2 (range: 15-83) yrs. The majority of patients $(68.4 \%)$ had no significant co-morbidity, and about one third of patients had at least one co-morbidity at presentation (cardial $11.1 \%$, pulmonal $4.3 \%)$.

\section{Neoadjuvant therapy}

Nine patients underwent preoperative $5 \times 5$ Gy, 40 patients received a long course RT, 53 patients had a long course CRT and 15 patients did not undergo neoadjuvant therapy ( 2 because of a history of pelvic irradiation).

\section{Type of resection}

In the 91 patients who were treated with curative intent, $74 \%$ underwent an 'extended TME'. In these patients the resection plane was beyond the mesorectal fascia in at least one area. Table 7.1 shows the types of resections carried out in these patients, and table 7.2 shows the resected structures for the patients who did not undergo a pelvic exenteration. In one patients with a solitary liver metastasis, a 'liver-first' approach was chosen, in which the patient first underwent neoadjuvant and surgical treatment for his liver metastasis, followed by resection of the rectum at a later stage. In 20 patients, postoperative brachytherapy was applied because of an anticipated narrow or involved resection margin. 
Locally Advanced Rectal Cancer

\begin{tabular}{lc}
\hline & N (of which extended TME) \\
\hline Abdomino Perineal Resection & $37(28)$ \\
Low Anterior Resection & $44(24)$ \\
Hartmann's procedure & $8(5)$ \\
Posterior/total pelvic exenteration & 28 \\
Total & 117 \\
\hline Table 7.1 Types of resections carried out. For APR, LAR and Hartmann's procedure, numbers in parenthesis show the \\
number of resections in which an extended TME was performed, i.e. a resection plane outside the mesorectal fascia \\
was chosen
\end{tabular}

\section{Adjuvant therapy}

Adjuvant chemotherapy was given in 55 patients (47\%). One-third of all patients who should have received adjuvant chemotherapy did not receive it, mostly because of postoperative complications.

\begin{tabular}{lc}
\hline Resected organs & N \\
\hline Vagina/posterior vaginal wall & 12 \\
Uterus and/or ovaries & 14 \\
Prostate (or part of) & 5 \\
Ureter & 9 \\
Seminal vesicle (1 or 2) & 11 \\
Bladder & 5 \\
Sacral bone & 4 \\
Coccygeal bone & 8 \\
Pelvic side wall and/or iliac vessels & 13 \\
Small bowel & 7 \\
\hline Table 7.2 Resected organs and structures in the patients that did not under- \\
go a pelvic exenteration
\end{tabular}

\section{Histopathology of the resection specimen}

Distribution of tumor stages found at histopathology is shown in table 7.3. Six patients had a pathological complete response to neo-adjuvant treatment ( 2 after long course CRT and 4 after long course RT). One of these six complete responses died from a non-small cell lung carcinoma, the other patients are alive and recurrence-free. The resection was microscopically complete (Ro) in 103 patients (88\%). There were significantly more complete resections $-96 \%(87 / 91)$ in the patients treated with curative intent, as compared to the patients who underwent a palliative resection $-62 \%(16 / 26)-(p=0.000)$. Eleven patients $(9 \%)$ had a microscopically incomplete resection (R1), and in 3 patients $(2.5 \%)$ there was a macroscopically incomplete resection $\left(R_{2}\right)$. 


\begin{tabular}{|c|c|}
\hline Stage at histology & $N$ \\
\hline (y)p $\mathrm{T}_{0} \mathrm{~N}_{0} \mathrm{M}_{\mathrm{o}}$ & 6 \\
\hline (y)pT $T_{1-2} N_{0} M_{0}$ & 8 \\
\hline (y)p $T_{3-4} \mathrm{~N}_{0} \mathrm{M}_{0}$ & 50 \\
\hline (y) $p T_{1-4} N_{1-2} M_{0}$ & 24 \\
\hline (y)pT $\mathrm{T}_{1-4} \mathrm{~N}_{\mathrm{O-2} 2} \mathrm{M}_{1}$ & 28 \\
\hline unknown & 1 \\
\hline Total & 117 \\
\hline
\end{tabular}

\section{Perioperative period}

Median hospital stay was 18 (range: 6-249) days. Thirty-five patients suffered major complications, which often needed surgical intervention (anastomotic leakage, postoperative haemorrhage, fascia dehiscence, bowel necrosis, sepsis). Another 44 patients had only minor complications e.g. superficial wound infection, urinary tract infection. Thirty-six patients underwent renewed surgery for their complications (any surgical intervention ranging from drainage of a wound abcess to relaparotomy). A total of 19 patients were readmitted after initial hospital stay because of postoperative complications. Mortality in the first 30 days after initial surgery was $2.6 \%$.

\section{Follow up}

Local recurrence-free, distant recurrence-free and overall survival are shown in figures 7.1-7.3. At a median follow-up of 52 (range: 13-120) months there were 5 local recurrences, 4 of which in patients treated with curative intent. Of the 87 patients treated with curative intent in which an Ro resection was achieved, 85 (97.7\%) did not develop a local recurrence. Characteristics of the patients who developed a local recurrence are shown in table 7.4.

In the group of patients treated with curative intent, 20 patients developed one or more distant recurrences (lung $9 ;$ liver $9 ;$ abdomen $4 ;$ cerebrum 1, other 2 ). Six of these 20 patients underwent treatment with curative intent of the metastatic disease, including surgical resection. Four patients are presently alive without evidence of disease 13.9 (range: 1.7-25.1) months after diagnosing the metastases. 


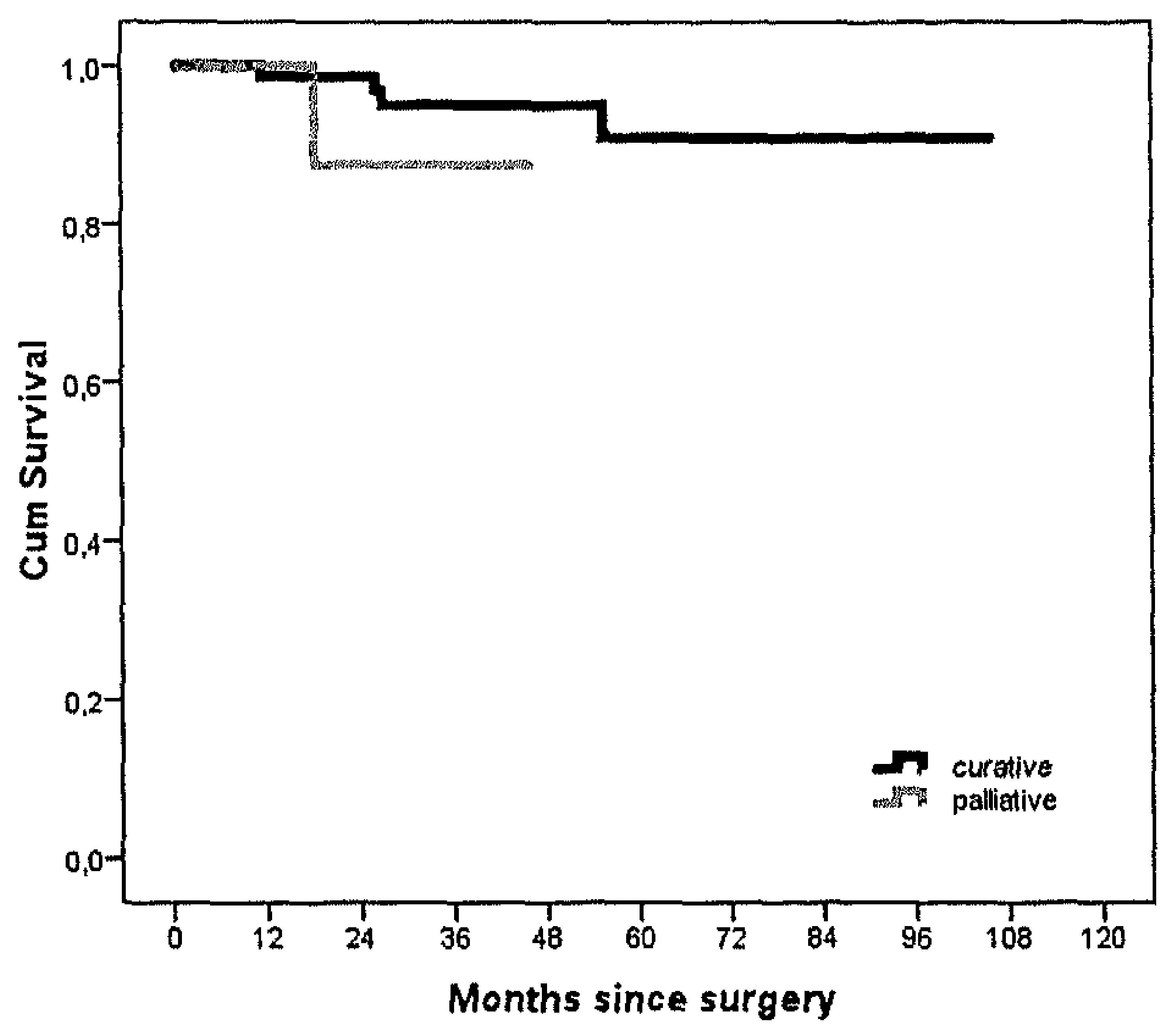

\begin{tabular}{llllll}
$\begin{array}{l}\text { Months since } \\
\text { surgery }\end{array}$ & 0 & 24 & 48 & 72 & 96 \\
\hline $\begin{array}{l}\text { Numbers at risk } \\
\text { Curative }\end{array}$ & 91 & 59 & 29 & 13 & 4 \\
$\begin{array}{l}\text { Palliative } \\
\text { Nutive }\end{array}$ & 26 & 4 & 0 & 0 & 0
\end{tabular}

Figure 7.1 Local recurrence free survival for curative vs. palliative resections of locally advanced rectal tumors including numbers at risk

\section{DISCUSSION}

This study shows that for locally advanced primary rectal cancer an aggressive multimodal treatment in a referral center achieves an excellent local control of $90 \%$ and an overall survival of $65 \%$ at 5 years. A comparison of the results of the present study with other series is hampered by a lack of a uniform definition of locally advanced tumors, and by the inclusion in some series of recurrent rectal cancer. Two large randomized series of locally advanced rectal cancer have reported local recurrence rates of $6-17 \%$ and overall survival of $65-75 \%^{16,17}$. These series however had defined locally advanced as all $\mathrm{T}_{3-4}$ and/or $\mathrm{N}_{+}$, instead of only those $T_{3}$ tumors with predicted close or involved margin, and therefore included mainly less advanced cases than the present series. More comparable series are 


\section{Chapter 7}

reported by Braendengen et al., who showed a local control of 82 and $67 \%$ at 5 years in a group of $\mathrm{CT} 4$ rectal cancer patients who underwent preoperative CRT or RT respectively, with an overall survival of 66 and $53 \%{ }^{18}$. Palmer et al. showed a local recurrence rate of $21 \%$ ( $14 \%$ in curative resections and $29 \%$ in non-curative resections) at a median follow-up of 63 months in a group of patients with locally advanced rectal cancer treated with multimodality treatment. The 5 year overall survival in the patients treated with curative intent was $34 \%^{19}{ }^{20}$. A study of Gosens et al. on the importance of obtaining a free CRM showed a 2 year LR-rate of 8 vs. $43 \%$ (CRM- vs. CRM+) in locally advanced rectal cancer treated with multimodality treatment, with an overall survival of $80 \mathrm{vs.} 58 \%$ (at 2 years) ${ }^{20}$. The local control rate of our study compares favorably with studies described above. Most likely the centralization of locally advanced rectal cancer treatment in a tertiary referral center and the aggressive multimodality approach play an important role in this good results. The overall survival however remains troublesome in all series.

\section{Imaging}

116 Imaging has an important role in the management of rectal cancer. In addition to endorectal ultrasound, $C T$ and especially MRI have been shown to provide detailed information on the exact extent of the tumor ${ }^{1,21-24}$. For locally advanced tumors that invade adjacent organs MRI gives the best anatomical information ${ }^{25}$. In our experience a high quality MRI is essential both for optimal surgical planning preoperatively, and for executing the procedure in the operating room.

\section{Neoadjuvant therapy}

Several studies investigated the use of various regimens of chemotherapy in addition to radiotherapy in the multimodality treatment of locally advanced cancer. Sauer et al. showed the benefit of neoadjuvant instead of adjuvant CRT (with 5 -FU) on the local recurrence rate ( 6 vs. $13 \%$ at 5 years) ${ }^{16}$, and Bosset et al. confirmed that the addition of chemotherapy (5-FU/Leucovorin) improves LR free survival ${ }^{17}$. Smaller phase $I / I$ trials have shown that the addition of oxaliplatin or irinotecan to 5 -FU or capecitabine results in a better pathological response ${ }^{2}$, and current trials are investigating the effect of biological response modifiers 
such as bevacizumab ${ }^{3}{ }^{4}$. It remains to be determined whether the higher response rates with these agents also lead to an improved clinical outcome.

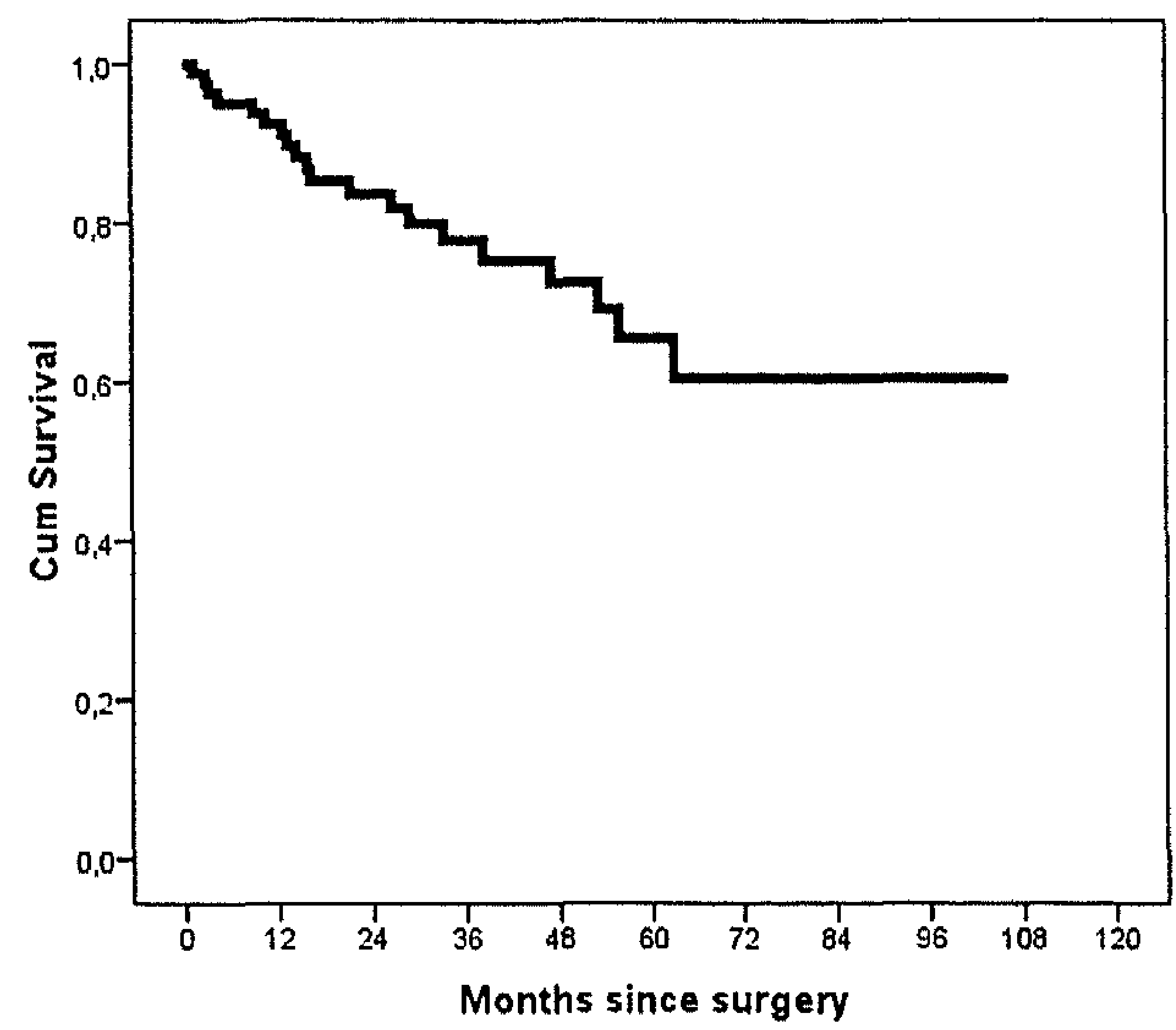

\begin{tabular}{lccccc}
$\begin{array}{l}\text { Months since } \\
\text { surgery }\end{array}$ & 0 & 24 & 48 & 72 & 96 \\
\hline Numbers at risk & 91 & 52 & 25 & 11 & 3
\end{tabular}

Figure 7.2 Distant recurrence free survival for curatively treated locally advanced rectal tumors including numbers at risk

\section{Surgical procedure}

A complete resection (Ro) has repeatedly been shown to be the most crucial factor to obtain a good outcome $\mathrm{e}^{5,20,26,27}$. Therefore the surgical procedure itself is probably the key factor for success. In literature, Ro rates for patients with locally advanced rectal cancer are $79-91 \%^{16-20}$. The $96 \%$ Ro (margin $>1 \mathrm{~mm}$ ) rate in our series for the patients treated with curative intent is most likely the reason for the high local control rate. This high Ro rate was the result of a very aggressive surgical approach. Whenever there was doubt on whether or not a plane was involved after neoadjuvant therapy, the approach generally was to consider it as involved, leading to an en-bloc resection of the area. Only in frail patients or in 
Chaptery

patients with severe co-morbidity a good response to CRT or RT sometimes led to a less extenswe procedure than originally planned

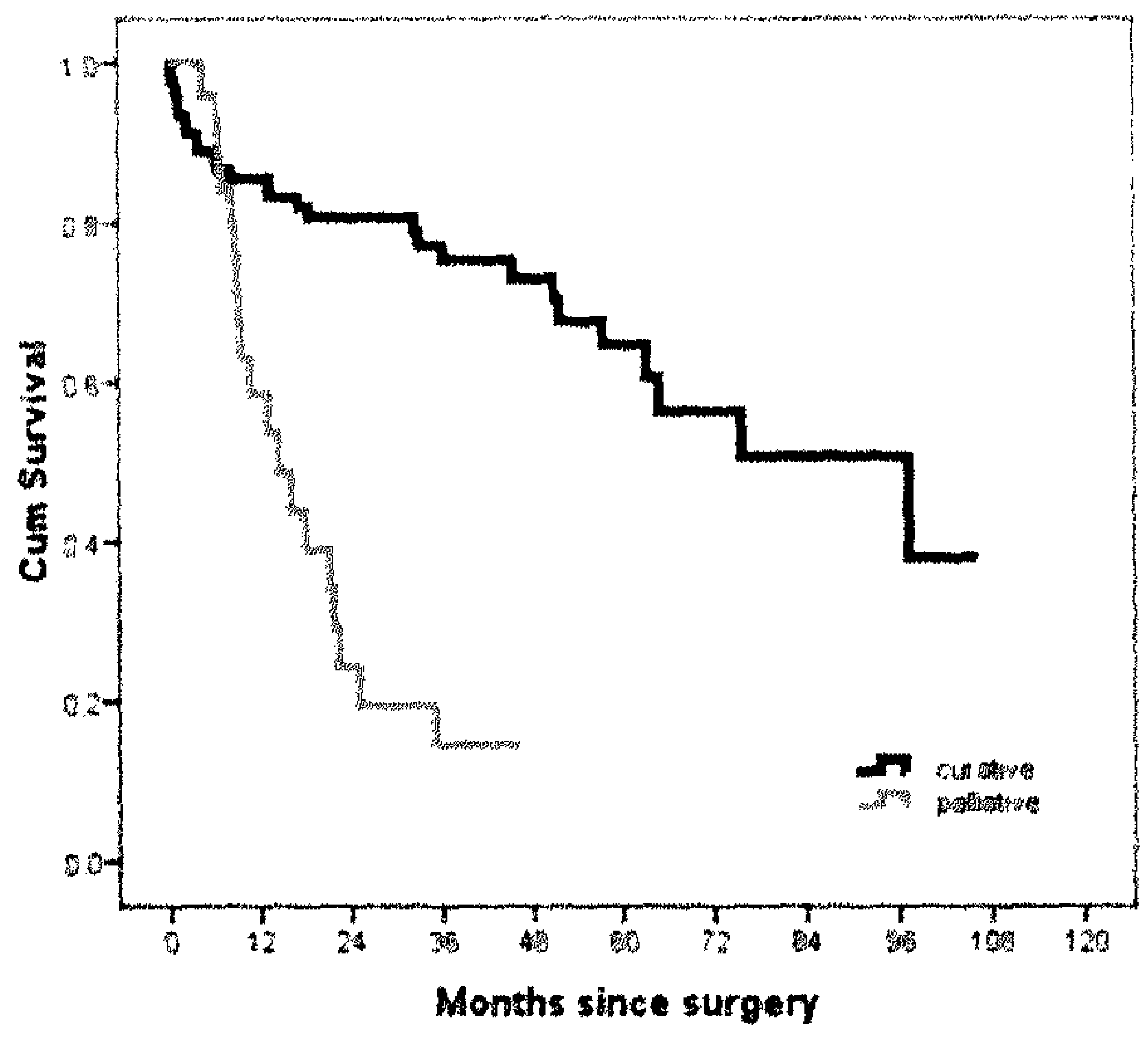

\begin{tabular}{|c|c|c|c|c|c|}
\hline $\begin{array}{l}\text { Months since } \\
\text { surgery }\end{array}$ & 0 & 24 & 48 & 72 & 96 \\
\hline \multicolumn{6}{|c|}{ Nombers at risk } \\
\hline Curative & 91 & 60 & 30 & 13 & 4 \\
\hline Potliative & 26 & 5 & 0 & 0 & 0 \\
\hline
\end{tabular}

Foure 7.3 Overall semwal for curative ws palliative resectrons of locally advanced rectal tumors including numbers at resk

\section{Additional radiotherapy}

Intraoperative radiotherapy (IORT) or brachytherapy is often advocated for locally advanced rectal cancer when it is difficult to obtain a complete resection. This strategy of intra-operative therapy at a dose of 10-20 Gy is especially valuable when it is possible to obtain margins which are microscopically incomplete, but without gross residual disease $e^{8-21,28}$. For primary locally advanced rectal cancer it is usually possible to obtain a tumor free margin, provided the surgeon is willing and able to perform a wider resection outside the normal resection plane. In the present series brachytherapy was mainly applied posterior 
and posterolateral from the level of the promontory to $S_{3}$, an area where it can be difficult to obtain a wide resection margin.

\begin{tabular}{|c|c|c|c|c|c|}
\hline & Patient 1 & Patient 2 & Patient 3 & Patient 4 & Patient 5 \\
\hline Cur/pal resection & curative & curative & curative & palliative & curative \\
\hline Preop. therapy & RT & - & CRT & RT & - \\
\hline Resection & total P.E. & total P.E. & total P.E. & Hartmann & extended LAR \\
\hline Postop. therapy & brachy & chemo & brachy & - & chemo \\
\hline Ro/R1 & $\mathrm{R}_{1}$ & Ro & $R_{I}$ & $\mathrm{R}_{1}$ & Ro \\
\hline Stage & ypT 4 No & $\mathrm{pT}_{4} \mathrm{Nz}$ & ypT 4 No & урт $T_{3} \mathrm{~N}_{1}$ & $\mathrm{pT}_{3} \mathrm{~N}_{1}$ \\
\hline Distant & & & & & \\
\hline recurrence & - & $t$ & - & - & - \\
\hline Treatment LR & surgery & surgery & - & - & surgery \\
\hline Survival & - & - & - & - & + \\
\hline
\end{tabular}

\section{Complications}

The downside of the aggressive approach in the present study is a high complication rate. About one third of patients developed major postoperative complications. A recent editorial on the treatment of locally advanced rectal cancer stated that a complication rate of $25-50 \%$ was probably realistic ${ }^{29}$. Because of the prolonged in-hospital stay and reconvalescence one third of the patients in the present series did not receive the planned adjuvant systemic therapy. This was also the experience of Braendengen et al. who reported a failure to administer adjuvant chemotherapy in half of the patients ${ }^{18}$. In addition to immediate postoperative complications the aggressive treatment of this disease is likely to cause long term functional anorectal and genitourinary morbidity. The good oncological outcome in the present study justifies this morbidity, although the good responses to the currently improving chemoradiation schedules may lead to a reconsideration of the present surgical strategy. In a patient with a good response less aggressive surgery could lead to an equally good oncological outcome with less morbidity. The challenge here is how best to assess a good response. 


\section{Chapter 7}

\section{Metastatic disease}

While the multidisciplinary efforts result in a very good local control, many patients still die of metastatic disease. At the time of diagnosis about $25 \%$ of patients with locally advanced rectal cancer present with metastatic disease, of whom only a minority can be cured. In $75 \%$ of patients without obvious metastases the 5 year actuarial metastatic rate is $40 \%$, with again only a minority that can be cured. This is a reflection of the presence of small volumes of metastatic disease at the time of diagnosis, the majority of is which is beyond the detection range of even the best of the current imaging techniques. This means that if we would like to make further improvements in overall survival in patients with (locally advanced) rectal cancer, the control of distant metastases has to be improved ${ }^{30}$. Both Bosset et al. and Sauer et al. did not show an overall survival benefit for the addition of chemotherapy to RT. This was also not shown in the study of Braendengen et al., although there seems to be a trend towards a better OS in the latter study $(p=.09)^{16-18}$. The results in these studies were obtained with the use of 5 -FU based chemotherapy. To achieve a better overall survival, two important steps could be considered. First, the effect of more effective new chemotherapeutic agents should be evaluated. Second, as one third to half of patients are not fit enough to receive chemotherapy postoperatively because of perioperative complications, administering all systemic therapy before the operation should seriously be considered. Some studies along these lines have been initiated ${ }^{31}$ and more studies will be required to establish the optimal schedule and timing of radiotherapy and systemic therapy.

\section{Conclusion}

In conclusion, this study demonstrates that excellent local control can be obtained in locally advanced primary rectal cancer with the use of aggressive multimodality treatment, at the expense of considerable morbidity. However, many patients still die of metastatic disease, and future research should be focused on systemic treatment regimens for better distant control. 


\section{Locally Advanced Rectal Cancer}

\section{REFERENCES}

1. Beets-Tan RG, Beets GL, Vliegen RF, Kessels AG, Van Boven $H$, De Bruine A, von Meyenfeldt MF, Baeten $C G$, van Engelshoven JM. Accuracy of magnetic resonance imaging in prediction of tumourfree resection margin in rectal cancer surgery. Lancet 2001;357(9255): 497-504.

2. Rodel $C$, Sauer R. Integration of novel agents into combined-modality treatment for rectal cancer patients. Strahlenther Onkol 2007;183(5): 227-235.

3. Rodel C, Arnold D, Hipp M, Liersch T, Dellas K, lesalnieks I, Hermann RM, Lordick F, Hinke A, Hohenberger W, Saver R. Phase I-ll trial of cetuximab, capecitabine, oxaliplatin, and radiotherapy as preoperative treatment in rectal cancer. Int J Radiat Oncol Biol Phys 2008;70(4): 1081-1086.

4. Czito BG, Bendell JC, Willett CG, Morse MA, Blobe GC, Tyler DS, Thomas J, Ludwig KA, Mantyh CR, Ashton J, YU D, Hurwitz HI. Bevacizumab, oxaliplatin, and capecitabine with radiation therapy in rectal cancer: Phase I trial results. Int J Radiat Oncol Biol Phys 2007;68(2): 472-478.

5. Nagtegaal ID, Quirke P. What is the role for the circumferential margin in the modern treatment of rectal cancer? J Clin Oncol 2008;26(2): 303-312.

6. Mannaerts GH, Rutten HJ, Martijn H, Groen GJ, Hanssens PE, Wiggers T. Abdominosacral resection for primary irresectable and locally recurrent rectal cancer. Dis Colon Rectum 2001;44(6): 806-814.

7. Melton GB, Paty PB, Boland PJ, Healey JH, Savatta SG, Casas-Ganem JE, Guillem JG, Weiser MR, Cohen AM, Minsky BD, Wong WD, Temple LK. Sacral resection for recurrent rectal cancer: analysis of morbidity and treatment results. Dis Colon Rectum 2006;49(8): 1099-1107.

8. Gunderson LL, Neison $H$, Martenson JA, Cha $S$, Haddock $M$, Devine R, Fieck JM, Wolff $B$, Dozois $R$, O'Connell MJ. Locally advanced primary colorectal cancer: intraoperative electron and external beam irradiation +1- 5-FU. Int J Radiat Oncol Biol Phys 1997;37(3): 601-614.

9. Harrison LB, Minsky BD, Enker WE, Mychalczak B, Guillem J, Paty PB, Anderson L, White C, Cohen AM. High dose rate intraoperative radiation therapy (HDR-IORT) as part of the management strategy for locally advanced primary and recurrent rectal cancer. Int J Radiat Oncol Biol Phys 1998;42(2): 325330.

10. Willett CG, Shellito PC, Tepper JE, Eliseo R, Convery K, Wood WC. Intraoperative electron beam radiation therapy for primary locally advanced rectal and rectosigmoid carcinoma. $J$ Clin Oncol 1991;9(5): 843-849.

11. Lanciano RM, Calkins AR, Wolkov HB, Buzydlowski J, Noyes RD, Sause W, Nelson D, Willett C, Owens $J C$, Hanks GM. A phase l/Il study of intraoperative radiotherapy in advanced unresectable or recurrent carcinoma of the rectum: a Radiation Therapy Oncology Group (RTOG) study. J Surg Oncol 1993;53(1): 20-29.

12. Wolpin BM, Meyerhardt JA, Mamon HJ, Mayer RJ. Adjuvant treatment of colorectal cancer. CA Cancer J Clin 2007;57(3): 168-185.

13. Klautke $G$, Fietkau R. Intensified neoadjuvant radiochemotherapy for locally advanced rectal cancer: a review. Int J Colorectal Dis 2007;22(5): 457-465.

14. Dirkx MJ, Huveneers JA, Botterweck AA, J.J. B, Bongaerts BW, Schouten LJ. Cancer incidence in Mid and South Limburg, the Netherlands 1986-2005. Maastricht: Comprehensive Cancer Centre Limburg; 2008.

15. Quirke $P$, Dixon MF. The prediction of local recurrence in rectal adenocarcinoma by histopathological examination. Int J Colorectal Dis 1988;3(2): 127-131.

16. Sauer R, Becker H, Hohenberger W, Rodel C, Wittekind C, Fietkau R, Martus P, Tschmelitsch J, Hager $E$, Hess CF, Karstens JH, Liersch T, Schmidberger $H$, Raab R. Preoperative versus postoperative chemoradiotherapy for rectal cancer. N Engl J Med 2004;351(17): 1731-1740.

17. Bosset JF, Collette $L$, Calais G, Mineur $L$, Maingon P, Radosevic-Jelic L, Daban A, Bardet E, Beny A, Ollier JC. Chemotherapy with preoperative radiotherapy in rectal cancer. $N$ Engl J Med 2006;355(11): 1114-1123.

18. Braendengen $M$, Tveit KM, Berglund A, Birkemeyer $E$, Frykholm $G$, Pahlman $L$, Wiig JN, Bystrom $P$, Bujko $K$, Glimelius B. Randomized phase III study comparing preoperative radiotherapy with chemoradiotherapy in nonresectable rectal cancer. J Clin Oncol 2008;26(22): $3687-3694$. 


\section{Chapter 7}

19. Palmer G, Martling A, Blomqvist L, Cedermark B, Holm T. Outcome after the introduction of a multimodality treatment program for locally advanced rectal cancer. Eur J Surg Oncol 2005i31(7): 727734 .

20. Gosens MJ, Klaassen RA, Tan-Go l, Rutten HJ, Martijn $\mathrm{H}$, van den Brule AJ, Nieuwenhuijzen GA, van Krieken JH, Nagtegaal ID. Circumferential margin involvement is the crucial prognostic factor after multimodality treatment in patients with locally advanced rectal carcinoma. Clin Cancer Res 2007;13(22 Pt 1): 6617-6623.

21. Beets-Tan RG, Beets GL. Rectal cancer: review with emphasis on MR imaging. Radiology 2004;232(2): 335-346.

22. Brown G, Richards CJ, Newcombe RG, Dallimore NS, Radcliffe AG, Carey DP, Bourne MW, Williams GT. Rectal Carcinoma: Thin-Section MR Imaging for Staging in 28 Patients. Radiology 1999;211(1): 215-222.

23. Blomqvist $L$, Rubio $C$, Holm $T$, Machado $M$, Hindmarsh $T$. Rectal adenocarcinoma: assessment of tumour involvement of the lateral resection margin by MRI of resected specimen. Br J Radiol 1999;72(853): 18.23.

24. Bissett IP, Fernando CC, Hough DM, Cowan BR, Chau KY, Young AA, Parry BR, Hill GL. Identification of the fascia propria by magnetic resonance imaging and its relevance to preoperative assessment of rectal cancer. Dis Colon Rectum 2001;44(2): 259-265.

25. Beets-Tan RG, Beets GL, Borstlap AC, Oei TK, Teune TM, von Meyenfeldt MF, van Engelshoven JM. Preoperative assessment of local tumor extent in advanced rectal cancer: $C T$ or high-resolution MRI? Abdom Imaging 2000;25(5): 533-541.

26. Nagtegaal ID, Marijnen CA, Kranenbarg EK, van de Velde CJ, van Krieken JH. Circumferential margin involvement is still an important predictor of local recurrence in rectal carcinoma: not one millimeter but two millimeters is the limit. Am J Surg Pathol 2002;26(3): 350-357.

27. Birbeck KF, Macklin CP, Tiffin NJ, Parsons W, Dixon MF, Mapstone NP, Abbott CR, Scott N, Finan PJ, Johnston $D$, Quirke $P$. Rates of circumferential resection margin involvement vary between surgeons and predict outcomes in rectal cancer surgery. Ann Surg 2002;235(4): 449-457.

28. Hu KS, Harrison LB. Results and complications of surgery combined with intra-operative radiation therapy for the treatment of locally advanced or recurrent cancers in the pelvis. Semin Surg Oncol 2000;18(3): 269-278.

29. Guillem JG. As in fly fishing, "matching the hatch" should govern the management of locally advanced rectal cancer. Ann Surg 2007;246(5): 702-704.

30. Glynne-Jones $R_{1}$ Mathur $P$, Elton C, Train ML. The multidisciplinary management of gastrointestinal cancer. Multimodal treatment of rectal cancer. Best Pract Res Clin Gastroenterol 2007;21(6): 10491070.

31. Chau I, Brown $G$, Cunningham $D$, Tait $D$, Wotherspoon $A$, Norman AR, Tebbutt N, Hill M, Ross PJ, Massey $A$, Oates J. Neoadjuvant capecitabine and oxaliplatin followed by synchronous chemoradiation and total mesorectal excision in magnetic resonance imaging-defined poor-risk rectal cancer. J Clin Oncol 2006;24(4): 668-674. 


\section{MRI after chemoradiotherapy of rectal cancer: a useful tool to select patients for local excision}

S.M.E. Engelen, R.G.H. Beets-Tan, M.J. Lahaye, G. Lammering, R.L.H. Jansen, R.M. van Dam, J. Konsten, J.W.A. Leiftens, M.F. von Meyenfeldt, C.J.H. van de Velde, G.L. Beets 
Chapter 8

\section{ABSTRACT}

Aim If identification of good responders to neoadjuvant chemoradiotherapy (CRT) in rectal cancer is possible, there might be opportunities for local excision in these good responders. The aim of this study was to determine if post-CRT MRI in rectal cancer can accurately identify ypTo-2/ypNo, because both features are essential for identification of good responders.

Methods Seventy-nine patients ( 4 hospitals) underwent post-CRT MRI, 62 received a lymph node-specific contrast agent. An expert and general radiologist prospectively predicted whether the tumor penetrated the mesorectal fat and whether nodes were sterilized after chemoradiation. Histology was the reference standard. Sens, spec, PPV and NPV were calculated; AUC's (for N stage) were constructed.

Results For prediction of whether a tumor penetrated the bowel wall, there was an NPV of 0.90 and 0.76 for the expert and general radiologist respectively. The AUC for prediction of nodal status was 0.90 and 0.72 with an NPV of 0.95 and 0.85 for expert and general radiologist respectively.

Conclusion This prospective multicenter study demonstrates that MRI interpreted by an expert radiologist can safely select ypTo-2/ypNo rectal cancer with low risk of undetected nodal metastases or invasion through the bowel wall. These patients could thus have been selected for local excision. However, future studies will have to prove equal outcome of such a modified surgical approach compared to current practice. 


\section{INTRODUCTION}

The standard surgical treatment for rectal cancer is total mesorectal excision ${ }^{1}$, or in case of a more advanced tumor, an even wider excision. Neoadjuvant radiotherapy, both in the form of a short course (SCRT) or chemoradiotherapy $(C R T)$, has been shown to improve the local control ${ }^{2-5}$, and is therefore considered standard practice in many countries. This multimodal treatment has resulted in a reduction in the number of local recurrences to $<10 \%$ at 5 years and has also increased the disease-free and the overall survival ${ }^{4-6}$. Therefore, from an oncological point of view, it can be considered as the standard treatment for the majority of patients.

Every treatment has its price: a total mesorectal excision is major abdominal surgery, with associated morbidity and mortality. Adding preoperative radiotherapy further increases wound healing problems and functional morbidity ${ }^{7-9}$. A local excision (or transanal endoscopic microsurgery - TEM) of the tumor however is known to only result in minimal morbidity and mortality, which comes at the expense of a potentially inferior oncological control, because most of the surrounding mesorectal fat with the lymph nodes is left in situ. Therefore, it can currently only be advocated in very small tumors, and in fragile patients who are not capable of undergoing major surgery ${ }^{10,11}$. Due to the effective neoadjuvant CRT however, rectal tumors are nowadays often downsized and the lymph nodes sterilized, and complete response rates ( $P C R$ ) of up to $25 \%$ have been reported ${ }^{12}$. Therefore, a local excision might be an attractive alternative for these good responders, who show no residual tumor deposits in the surrounding mesorectal fat or in the lymph nodes, because it would then combine minimal morbidity with adequate oncological control. Lezoche et al. compared in a randomized study the local control rates of local excision in patients with T2-3No tumors with laparoscopic TME, both after CRT, and found a comparable local failure rate of only $5 \%$ for both arms with a median follow up of 55 months $^{13}$.

In general, a minimally invasive surgical approach such as local excision could only be safely administered when the patients have been accurately selected preoperatively. Ideally only those patients would be selected for local excision, in whom the tumor is limited to the bowel wall after CRT, and even more importantly in whom all lymph nodes have been sterilized. But as with primary 


\section{Chapter 8}

staging, MRI staging after CRT has only shown a limited accuracy, for both T-and $\mathrm{N}$ - stag $\mathrm{e}^{14-16}$. With regard to the nodal staging with MRI, the lymph node-specific contrast agent USPIO (Ultrasmall Superparamagnetic Iron Oxide) has shown promising results in a variety of cancers ${ }^{17}$. This has also been tested in rectal cancer, with again good results and especially a high negative predictive value ${ }^{18}$. When USPIO-MRI also accurately predicts No status àfter CRT, the identification of patients in whom it is safe to consider a local excision becomes more realistic. The aim of this study is therefore to determine the accuracy of MRI after CRT of rectal cancer for the identification of tumors limited to the bowel wall and the assessment the lymph node status, both in general and experienced hands.

\section{METHODS}

\section{Patients}

Institutional review board approval from all participating centers and informed consent from all patients was obtained. Between February 2003 and January 1262008,296 patients with histologically proven primary adenocarcinoma of the rectum were included in a prospective multicenter project that investigated the effect of a stratified treatment in primary rectal cancer based on MRI criteria. Primary endpoints of the multicenter project were 1) the number of complete resections, compared to a historical cohort and 2) local recurrence free survival. All rectal tumors were defined by an inferior margin not farther than $15 \mathrm{~cm}$ from the anal verge as described by endoscopy. Patients underwent a USPIO- (Ultra Small Paramagnetic Iron Oxide - a lymph node specific contrast agent) MRI. Patients with tumor at high risk for local recurrence (criteria see below) were advised to receive CRT. Ninety-five patients underwent chemoradiation and were asked to undergo a second USPIO-MRI after CRT. Of the 79 patients who underwent a second MRI, 62 had a USPIO-MRI.

Patients were included in one university hospital (University Hospital Maastricht) and three general hospitals (Laurentius Hospital Roermond, Viecuri Medical Center Venlo and St. Jans Hospital Weert). Patients were excluded from the study in case of pregnancy, age below 18 years, a history of previous malignancy or a contra-indication for MR imaging (pacemaker, neurostimulator, insulin pump, certain vascular clips (e.g. used in brain surgery), cochlear implants, 
metallic splinters in the eye or any other metal implant not securely fixed or electronic device).

\section{Chemoradiotherapy}

Radiotherapy was applied for 28 fractions of $1.8 \mathrm{~Gy}$, only on weekdays. The concomittant chemotherapy consisted of oral capecitabine $\left(825 \mathrm{mg} / \mathrm{m}^{2}\right.$ twice a day for 7 days a week) during the entire period of the radiotherapy. Furthermore most patients received one course of oxaliplatin/capecitabine (oxaliplatin 130 $\mathrm{mg} / \mathrm{m}^{2}$ on day 1 ) during the $6-8$ week period after radiotherapy preceding surgery.

\section{Ultrasmall Superparamagnetic Iron Oxide}

Ultrasmall Super Paramagnetic Iron Oxide (USPIO) is a lymph node specific contrast agent. The USPIO MR contrast agent (Sinerem, Guerbet Laboratories, Roissy, France) consists of low molecular weight iron oxide coated with dextran. Sinerem is supplied as a powder which needs to be reconstituted using saline and administered at a dose of $2.6 \mathrm{mg} \mathrm{Fe} / \mathrm{kg}$. USPIO was administered by slow intravenous infusion within a period of 45 minutes, thirty-six to twenty-four hours before the MRI scan took place. Patients were closely monitored for any adverse effects during and shortly after the infusion.

The nanoparticles iron oxide accumulate in macrophages within the lymph node and cause a decrease in signal intensity within the node due to susceptibility artefacts on $\mathrm{T}_{2}{ }^{*}$-weighted images ${ }^{19}$. This means that also enlarged inflamed nodes will be depicted as nodes with considerable signal decrease on $T_{2} *_{-}$ weighted images. The metastatic part of the lymph node will show no signal decrease and will be depicted as a white region within the lymph node due to the replacement of macrophages by tumor cells.

\section{MR Imaging}

MR imaging was performed on a 1.0/1.5T system (see methods and table 5.1 of chapter 5 for scan parameters) using a pelvic phased array coil with the patient in a feet first, supine position. Sequences used are sagittal and axial 2D T2W TSE, axial $3 D$ T2 $* W$ GRE and axial $3 D$ TIW GRE. Patients did not receive bowel or other preparation. Total scan time was approximately 40 minutes. 


\section{Chapter 8}

Postchemoradiation MRI was performed one to two weeks before surgery (i.e. about 6 weeks after the last fraction radiation therapy).

\section{Image evaluation}

All MR images made in the regional centers were immediately sent to the university hospital. The MR images were prospectively read by the local general radiologist with no specific experience in reading pelvic MRI and independently double read by an experienced abdominal MR radiologist ( $>10$ years experience in reading pelvic MRI) from the university hospital. The T-stage, N-stage, circumferential resection margin and height of the tumor were predicted by both radiologists. Based on the MR findings of the expert radiologist the patients were stratified into different risk groups for local recurrence. High risk was defined as: distance from the tumor to mesorectal fascia $\leq 2 \mathrm{~mm}$ or a $\mathrm{T}_{3}$ tumor that was localized in the distal third below $6 \mathrm{~cm}$ from the anal verge, or any tumor with more than 3 predicted lymph node metastasis (N2). On the second MRI, after CRT, the assessment of T-stage was performed on standard axial images ( $T_{2}$ weighted images). Radiologists were asked to predict the tumor stage from $\mathrm{T}_{1}$ to $T_{4}$. As only the distinction between T1-2 tumors (confined to the bowel wall) and T3-4 tumors (penetrating through the bowel wall) was considered relevant in the decision process, the data were dichotomized along this distinction. A tumor was considered as T1-2 (limited to the bowel wall) when the bowel wall was depicted as an intact line of low signal intensity. For nodal staging, the expert and general radiologist assigned a confidence level interval ( $0=$ definitely No to $4=$ definitely $\mathrm{N+}$ ) of the nodal status on patient basis in each patient twice; first based on the conventional T2-weighted images and thereafter on the combined conventional T2-weighted images and the USPIO-enhanced $\mathrm{T}_{2}$ *-weighted images. Both readers were blinded for each other's results. On non-enhanced T2-weighted images the short and long axis of the node, border irregularity and homogenous signal intensity were used as criteria for nodal prediction. On USPIO-enhanced $\mathrm{T}_{2}{ }^{*}$-weighted images radiologists used the estimated percentage of white region within the node, which was found to be a reliable criterion for identifying metastatic nodes ${ }^{20}$. 


\section{Surgical treatment}

Patients underwent surgery 6-8 weeks after the last fraction of radiotherapy. A standard TME was performed if possible after CRT, however more often an enbloc wider resection of surrounding tissues was necessary. The surgical resection plan was usually based on the MRI before the CRT, although sometimes with a very good response on the MRI after CRT this plan was modified to a less extensive resection.

\section{Histopathological evaluation}

The resection specimen was evaluated in a standardized way, as described by Quirke $^{21}$ : (1) the specimen was fixed in formalin for $24-48 \mathrm{hrs}$, (2) the circumferential resection plane was inked, (3) the specimen was sliced transversely every $5 \mathrm{~mm}$, to determine the location of the smallest distance from tumor to resection plane and to search for lymph nodes, (4) lymph nodes were processed according to standard methods and stained with haematoxylin-eosin. The pathologist evaluated $T$ stage, the circumferential resection margin (CRM), nodal status, distance of the tumor to proximal and distal resection margin and completeness of the resection (Ro/R1) on a patient basis.

\section{Statistical analysis}

Prediction of T-stage (limited to the bowel wall vs. penetrating the perirectal fat) and nodal status (positive vs. negative) on postchemoradiation (USPIO) MRI by an expert radiologist and general radiologists as one group was compared to ypT and ypN status. For T-stage (tumor limited to the bowel wall or not) sensitivity, specificity, PPV and NPV were calculated from a $2 \times 2$ contingency table. For nodal status, ROC curves were constructed and the Area Under the Curve (AUC) was calculated to determine the diagnostic performance of the expert and general radiologist. The cut-off value for ROC curve was chosen between 2 and 3 . The constructed ROC curves were compared for non-enhanced vs. USPIOenhanced and expert vs. non-expert $N$ stage prediction and P-values were calculated using the Delong, Delong and Clarke-Pearson method ${ }^{22}$. Sensitivity, specificity, PPV and NPV were calculated for the expert as well as the general radiologist, with the cut-off value between 2 and 3 for the definition of a positive node. Interobserver agreement for expert vs. non-expert was given using a 


\section{Chapter 8}

square weighted Kappa, with a 95\%-Confidence Interval. Analyses were performed using SPSS (version 12.0.1, 2003; SPSS, Chicago, III.). The square weighted Kappa's were calculated using STATA software (Stata Statistical Software: Release 9. College Station, TX: StateCorp LP).

\section{RESULTS}

Seventy-nine patients (52 male patients, median age 65 (range $35^{-86}$ )) underwent an MRI after CRT immediately preceding surgery, and provided data to evaluate ypT stage. On the histopathological specimen, in 16/79 patients no residual tumor was detectable (ypTo) and in 23/79 patients the tumor was confined to the bowel wall (ypT1-2), thus the total number of patients in which the tumor did not extend beyond the rectal wall after CRT was 39. In total, 62 patients underwent a USPIO-MRI after CRT and were further analyzed for ypN prediction. Of these 62 patients, 18 had a positive nodal status on histopathology $(y p N+)$. The mean number of lymph nodes harvested in the resection specimens was 7.5 (range: 0-29). Of the 16 patients with no residual tumor, 2 still presented with one or more positive lymph nodes, therefore 14 patients $(18 \%)$ in total responded with a complete pathological response (ypTo,ypNo).

\section{Prediction of tumor extent}

The accuracy figures are listed in table 8.1. Because the images are evaluated 'on the safe side', the sensitivity is higher than the specificity. The NPV, the prediction that the tumor remnant does not penetrate the muscle and is confined to the bowel wall, reached 0.90 for the expert radiologist and 0.76 for the general radiologists. There is no significant difference between the expert radiologist and the general radiologists for the overall accuracy $(p=0.12)$. When evaluating 1.0Tesla vs. 1.5Tesla, it was observed that the expert radiologist performed equally good on both field strengths, whereas the general radiologist showed a trend towards a better performance on 1.5Tesla MRI, although this was not significant $(p=0.30)$ (data not shown). The interobserver agreement for the prediction of the T-stage was substantial ( $K=0.64$ (95\% C.I. $0.47-0.80)$ ). 
Restaging of Rectal Cancer after CRT

\begin{tabular}{lcc}
\hline $\begin{array}{l}\text { Detection of } \\
\text { ypT3-4 }\end{array}$ & $\begin{array}{c}\text { University Setting } \\
n=79\end{array}$ & $\begin{array}{c}\text { General Setting } \\
n=79\end{array}$ \\
\hline Acc & 0.71 & 0.63 \\
\hline Sens & 0.95 & 0.88 \\
& $(38 / 38+2)$ & $(35 / 35+5)$ \\
\hline Spec & 0.46 & 0.41 \\
& $(18 / 18+21)$ & $(16 / 16+23)$ \\
\hline PPV & 0.64 & 0.60 \\
& $(38 / 38+21)$ & $(35 / 35+23)$ \\
\hline NPV & 0.90 & 0.76 \\
& $(18 / 18+2)$ & $(16 / 16+5)$ \\
\hline
\end{tabular}

Table 8.1 shows the Accuracy (acc), sensitivity (sens), specificity (spec), PPV and NPV for tumors still penetrating

the perirectal fat after chemoradiation in university and general setting

\section{Prediction of nodal status}

The AUCS for the prediction of nodal status on non-enhanced and USPIOenhanced MRI were 0.81 and 0.90 respectively for the expert radiologist and 0.67 and 0.72 for the general radiologist (see figure 8.1).

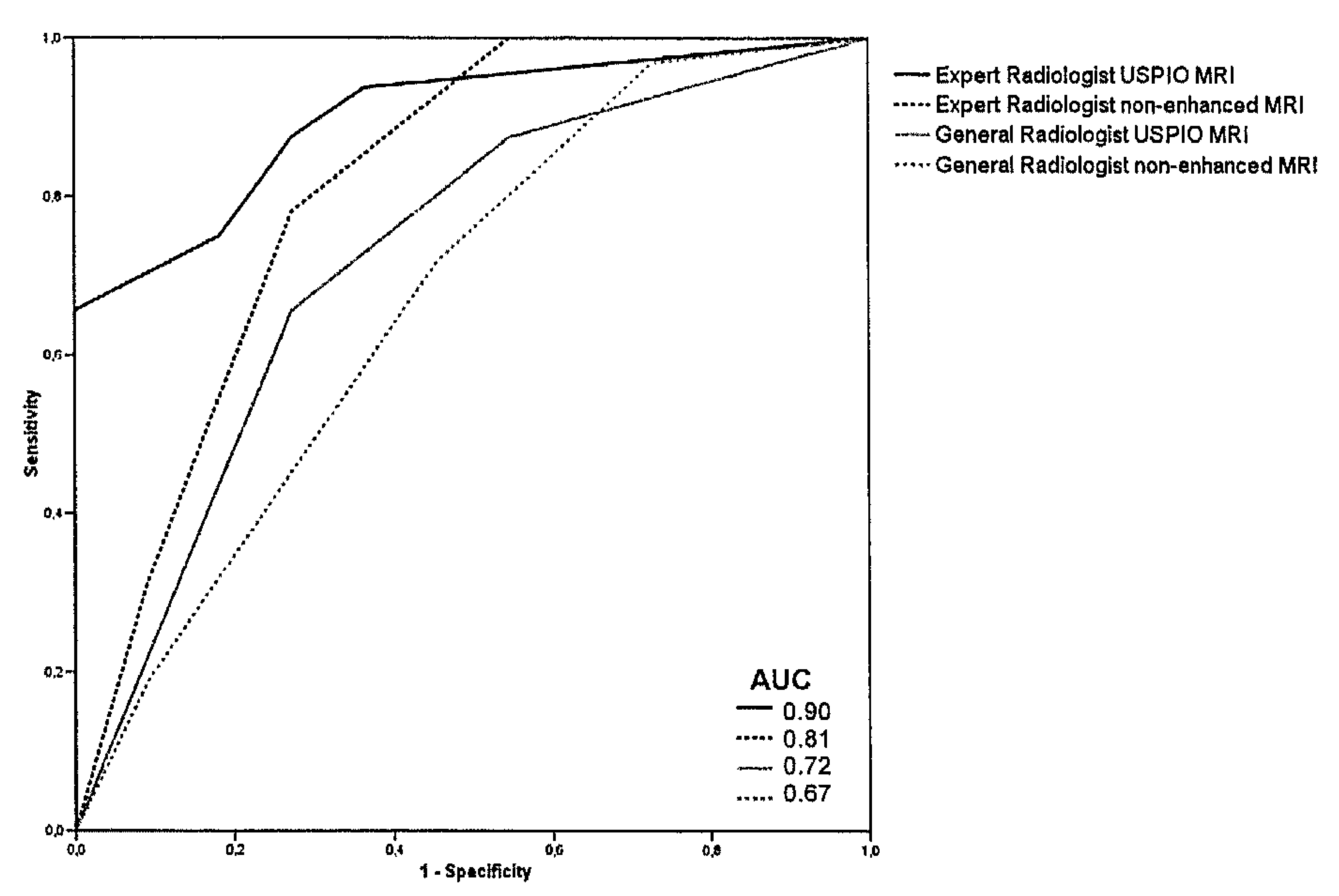

Figure 8.1 Receiver operating characteristic curves and AUCS for the expert and the general radiologists for prediction of nodal status postchemoradiation, on USPIO-enhanced and non-enhanced MR images 


\section{Chapter 8}

There was no statistically significant difference between the AUC when evaluating 1.0Tesla vs 1.5Tesla. The sensitivity, specificity, PPV and NPV of the prediction of both expert and general radiologist are listed in table 8.2. The NPV for prediction of nodal status with USPIO-enhanced MRI was 0.95 and 0.85 for the expert and general radiologist respectively (see figure 8.2). For both the expert and the general radiologist, the differences in diagnostic performance (AUC) between USPIO-enhanced and non-enhanced MRI were small and did not reach statistical significance (expert: $p=0.59$; general radiologist: $p=0.88$ ). However, when USPIO-enhanced images were evaluated, the expert performed better than the non-expert $(p=0.004)$. The interobserver agreement for prediction of $\mathrm{N}$ stage was substantial on USPIO-enhanced ( $K=0.60$ ( $95 \%$ C.I. 0.39 $0.80)$ ) and moderate on non-enhanced $(K=0.53(95 \%$ C.I. $0.26-0.81))$ MRI.

\begin{tabular}{lcccc}
\hline & \multicolumn{2}{c}{ Non-enhanced MRI } & \multicolumn{2}{c}{ USPIO MRI } \\
Detection of ypN+ & University Setting & General Setting & University Setting & General Setting \\
& $n=53$ & $n=52$ & $n=62$ & $n=57$ \\
\hline AUC & 0.81 & 0.67 & 0.90 & 0.72 \\
$95 \% C I$ & $(0.64-0.98)$ & $(0.47-0.87)$ & $(0.80-0.99)$ & $(0.54-0.91)$ \\
\hline Sens & 0.67 & 0.64 & 0.89 & 0.73 \\
& $(10 / 10+5)$ & $(9 / 9+5)$ & $(16 / 16+2)$ & $(11 / 11+4)$ \\
\hline SpeC & 0.84 & 0.69 & 0.80 & 0.55 \\
& $(32 / 32+6)$ & $(25 / 25+11)$ & $(35 / 35+9)$ & $(23 / 23+19)$ \\
\hline PPV & 0.63 & 0.45 & 0.64 & 0.37 \\
& $(10 / 10+6)$ & $(9 / 9+11)$ & $(16 / 16+9)$ & $(11 / 11+19)$ \\
\hline NPV & 0.86 & 0.83 & 0.95 & 0.85 \\
& $(32 / 32+5)$ & $(25 / 25+5)$ & $(35 / 35+2)$ & $(23 / 23+4)$ \\
\hline
\end{tabular}

Table 8.2 shows the area under the ROC curve (AUC) and the corresponding sensitivity (sens), specificity (spec), PPV and NPV for the non-enhanced and USPIO-enhanced prediction of the nodal status after chemoradiation

\section{Prediction of yp T1-2No disease}

The prediction of a tumor that has become the equivalent of stage I disease after chemoradiation combines the prediction of $\mathrm{T}$-stage and $\mathrm{N}$-stage for identification of T1/2-No tumors. This combination leads to a sens, spec, PPV and NPV of 0.40 , $0.97,0.92$ and 0.66 , indicating considerable overstaging. However, the high PPV and specificity indicate that when the radiologist states the tumor has become yTo-2No, it is right in more than 9 out of ten cases. 

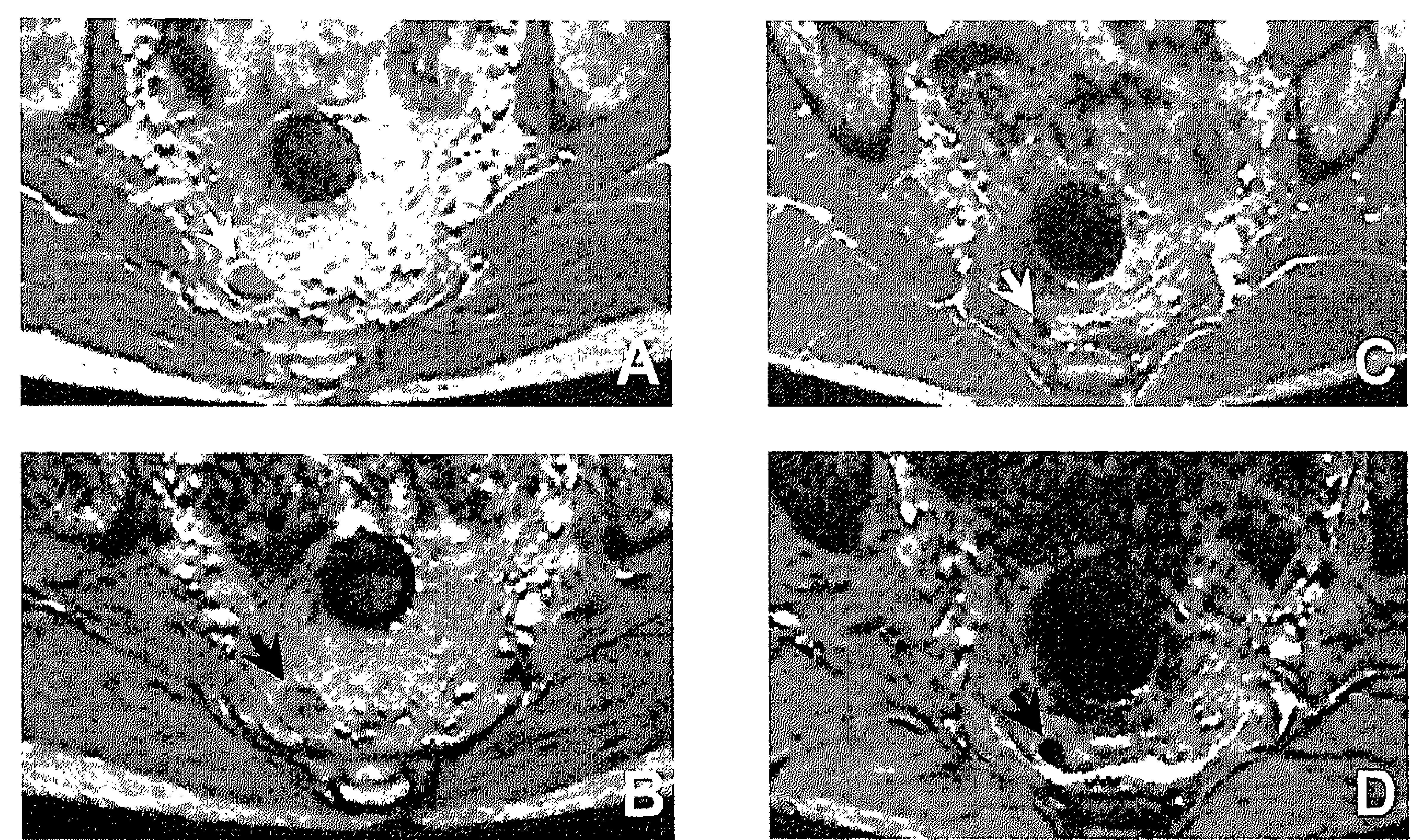

Figure 8.2 shows a lymph node pre-and postchemoradiation. (A) Pre-chemoradiation TIW MR image of a rectal cancer patient is used for the identification and localization of lymph nodes (white arrow). (B) On the $T_{2} * W M R$ image (post-USPIO image), the lymph node is hyperintense indicating a malignant node (black arrow). (C) TIW image of the same patient after chemoradiation is made again to localize the node (white arrow). (D) The node has now turned hypo-intense on the $T_{2} * W M R$ image, indicating sterilization of the node (black arrow) due to chemoradiation therapy. This sterilization of lymph nodes can be predicted with high NPV

\section{DISCUSSION}

This prospective study demonstrates that a dedicated MRI can predict with a reasonable degree of certainty whether the tumor remnant after CRT of a rectal cancer does not penetrate the rectal wall anymore and whether there are no malignant lymph nodes remaining. In the clinical context of selecting good responders to CRT for local excision a high negative predictive value of the MRI is important to minimize the risk of undertreatment. In the present study the NPV of the nodal status varied between $83 \%$ and $95 \%$, with relatively small differences depending on the use of contrast and the experience of the radiologist. In others words: when the radiologist states that the nodes are not malignant, this is be true in 8 to 9 times out of 10 . The predictive value of the identification of a tumor remnant confined to the rectal wall varied between $76 \%$ and $90 \%$ depending on 


\section{Chapter 8}

the experience of the radiologist. For the prediction of a tumor that has become the equivalent of stage I disease after chemoradiation, combining $T$ and $N$ prediction, the PPV was very high, with a lower NPV, indicating that when the radiologist states the tumor has become stage $\mathrm{I}$, it most likely is so.

Several earlier studies have investigated the accuracy of MRI as well as EUS for the prediction of $\mathrm{T}$ - and $\mathrm{N}$-stage in rectal cancer after CRT. The prediction of the exact T-stage is moderate for MRI with accuracies ranging from $43-60 \%^{14-16,23,24}$. This moderate accuracy is mainly related to the difficulty of postCRT MRI to differentiate between fibrosis only and fibrosis with viable tumor. EUS suffers from the same limitation with an overall accuracy of only $48-66 \%{ }^{25,}{ }^{26}$. Most of these studies assessed the ability to predict all $\mathrm{T}$-stages, and this may explain the higher accuracy of $63-71 \%$ in our study that focused only on the distinction between ypTo-2 and ypT3-4. In a previous retrospective MRI study from our research group on the same distinction we found an overall accuracy of $72-78 \%{ }^{27}$. For the assessment of nodal involvement after CRT there is one study in which no patient was understaged using EUS in experienced hands ${ }^{26}$. Another recent retrospective analysis demonstrated a high NPV of $93 \%$ with the use of MRI without contrast-enhancement ${ }^{23}$. In general it can be concluded however, that most studies in the literature have reported of an understaging of around $20 \%$ for both the standard MRI and the EUS ${ }^{14-16,23-26}$. It is especially the high NPV (and thus low number of understaged patients) in our present prospective multicenter study that suggests that the use of contrast-media can make MRI- based lymph node staging useful for safe clinical decision-making.

The general problem with lymph node imaging, regardless of the technique, lies in the difficulties to assess small nodes. The smaller the node, the harder it is to evaluate border and tumor heterogeneity. In rectal cancer, metastases are known to often present as very small nodes. In a study by Wang et al ${ }^{28}$ more than $50 \%$ of the involved nodes were between 2 and $5 \mathrm{~mm}$ in size. When using only size criteria on imaging, these small lymph nodes would be classified as benign. The advantage of contrast agents in MRI is that it provides both functional and morphological data for the identification of metastatic nodes. MRI with the lymph node specific contrast agent USPIO has shown to improve the sensitivity 
and specificity to $88 \%$ and $96 \%$ respectively in a meta analysis of studies performed at different cancers ${ }^{17}$. The data obtained so far for rectal cancer are also promising ${ }^{20,29}$. Our research group has studied criteria for nodal assessment after chemoradiation and found that the accuracy rates on a nodal basis were around $80 \%$ with conventional MRI and $95 \%$ with USPIO MRI (yet unpublished results). The present larger multicenter cohort study evaluates the accuracy for nodal staging in a clinically more useful per patient basis, with accuracy rates of $82 \%$, with especially a useful high NPV of $95 \%$. In the setting of our study there was only a minimal non-significant improvement in accuracy with USPIO when compared to standard MRI. This could be explained by the fact that after CRT lymph nodes smaller than $5 \mathrm{~mm}$ often disappear on MRI and are often sterilized by the CRT, making the 'conventional' size criteria more reliable.

In a previous study on the accuracy of MRI in predicting risk factors in a patient group that did not receive a long course of chemoradiation (chapter 5 ) we found a trend to a worse accuracy for 1.0Tesla MR machines as compared to 1.5Tesla MR machines for prediction of $T$ stage and nodal status. In the present study, the effect was less pronounced. Although we cannot draw definitive conclusions on this because our study was not properly designed to address this question, it seems prudent to be cautious in making important decisions on MR assessment of T and $\mathrm{N}$ stage on a 1.0 Tesla MR machine.

How much accuracy is required for safe clinical practice? In considering a local excision after a good response of a rectal cancer to chemoradiation, can clinicians accept an $80-90 \%$ negative predictive value? For the depth of invasion of the residual tumor the 10-20\% understaging does not form much of a clinical problem, because the residual tumor will still be histologically examined after local excision, and a re-resection could still be performed as a formal TME resection. For the nodal involvement however this question is more difficult to answer, because at a local tumor excision no mesorectal lymph nodes are removed, with an estimated $10 \%$ risk of leaving behind metastatic lymph nodes. This does not necessarily lead to a cancer death in $10 \%$ of patients. Some patients might die from metastatic disease unrelated to the lymph node that was left behind, and some patients might die from intercurrent disease, and in some patients adjuvant chemotherapy might be able to eradicate microscopic nodal 


\section{Chapter 8}

disease $^{5}$. In patients who do develop a local recurrence a strict MRI follow up protocol could detect the recurrence early, and a total mesorectal excision performed at that time might be curative. There will however be a small number of patients with a local recurrence who will suffer from the insufficiently provided local control with a local excision. This has to be balanced against the morbidity and mortality of the standard total mesorectal excision. In elderly patients and patients with severe co-morbidity many surgeons would consider the option of a local excision in good responders, based on MRI. For younger and fitter patients this strategy should at present be considered experimental, until proven safe in large studies.

With regard to minimizing surgical morbidity and mortality, one could even go one step further in patients in whom a clinical complete response is observed after CRT. Habr-Gama et al. reported a 93\% 5-year overall survival and a 85\% 5year disease free survival in a selected group of these patients with a wait-andsee policy ${ }^{30}$. The assessment of a complete response in the primary tumor is not easy, as it is difficult for all imaging methods to distinguish fibrosis without viable tumor from fibrosis with viable tumor ${ }^{31}$. In our current study we did not focus on the identification of a complete response in the primary tumor. The assessment of the nodal status after chemoradiation is however also important in these patients, as in a small percentage of patients with a complete response in the primary tumor, nodal disease is still present ${ }^{32}$. More clinical research is required before recommending a wait-and-see policy in complete responders in current clinical practice.

A limitation of our study is that in our treatment protocol only patients with relatively advanced tumors received CRT. Studies that address the issue of a local excision after CRT usually select patients with smaller tumors ${ }^{13 .}{ }^{33}$. In these patients more downstaging to ypTo-2No is expected due to the smaller tumor load. However, although the tumors in the present study were more advanced, we think that MRI will perform equally well in accurately staging the residual tumor and the lymph nodes after CRT in smaller tumors.

Another limitation is the practical difficulty with MR lymph node staging using USPIO: despite the promising results in nodal staging with USPIO described by 


\section{Restaging of Rectal Cancer after CRT}

Will et al. ${ }^{17}$ and our own good results in rectal cancer staging ${ }^{34}$, the USPIO contrast agent is not yet available on the market. It is however clear that with improving MR techniques and contrast agents MR lymph node imaging will become clinically relevant.

In conclusion, this prospective multicenter study demonstrates that with a dedicated MRI ypTo-2No rectal cancers after CRT can be accurately identified with a reasonably low risk of understaging. Thus, this diagnostic tool could help to select patients suitable for a local excision of the residual tumor. However, further research is needed to prove the safety and the equal outcome of such a modified surgical approach compared to current practice. 


\section{Chapter 8}

\section{REFERENCES}

Heald RJ, Ryall RD. Recurrence and survival after total mesorectal excision for rectal cancer. Lancet 1986;1(8496): 1479-1482. Improved survival with preoperative radiotherapy in resectable rectal cancer. Swedish Rectal Cancer Trial. N Engl J Med 1997;336(14): 980-987.

Kapiteijn E, Marijnen CA, Nagtegaal ID, Putter $H$, Steup WH, Wiggers T, Rutten $H J$, Pahlman L, Glimelius B, van Krieken JH, Leer JW, van de Velde CJ. Preoperative radiotherapy combined with total mesorectal excision for resectable rectal cancer. N Engl J Med 2001;345(9): 638-646.

Gerard JP, Conroy T, Bonnetain F, Bouche O, Chapet $O$, Closon-Dejardin MT, Untereiner M, Leduc B, Francois E, Maurel J, Seitz JF, Buecher B, Mackiewicz R, Ducreux M, Bedenne L. Preoperative radiotherapy with or without concurrent fluorouracil and leucovorin in T3-4 rectal cancers: results of FFCD 9203. J Clin Oncol 2006;24(28): 4620-4625.

Bosset JF, Collette L, Calais G, Mineur L, Maingon P, Radosevic-Jelic L, Daban A, Bardet E, Beny A, Ollier JC. Chemotherapy with preoperative radiotherapy in rectal cancer. $N$ Engl I Med 2006;355(11): 1114-1123.

Krook JE, Moertel CG, Gunderson LL, Wieand HS, Collins RT, Beart RW, Kubista TP, Poon MA, Meyers WC, Mailliard JA, et al. Effective surgical adjuvant therapy for high-risk rectal carcinoma. $N$ Engl J Med 1991;324(11): 709-715.

Marijnen CA, Kapiteijn E, van de Velde CJ, Martijn H, Steup WH, Wiggers T, Kranenbarg EK, Leer JW. Acute side effects and complications after short-term preoperative radiotherapy combined with total mesorectal excision in primary rectal cancer: report of a multicenter randomized trial. $J$ Clin Oncol 2002;20(3): 817-825.

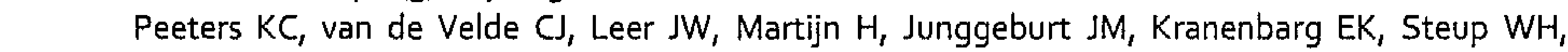
Wiggers $T$, Rutten HJ, Marijnen CA. Late side effects of short-course preoperative radiotherapy combined with total mesorectal excision for rectal cancer: increased bowel dysfunction in irradiated patients--a Dutch colorectal cancer group study. J Clin Oncol 2005;23(25): 6199-6206.

Valentini V, Beets-Tan R, Borras JM, Krivokapic Z, Leer JW, Pahlman L, Rodel C, Schmoll HJ, Scott $N$, Velde $C V$, Verfaillie $C$. Evidence and research in rectal cancer. Radiother Oncol 2008.

Ptok $H_{1}$ Marusch F, Meyer F, Schubert D, Koeckerling F, Gastinger I, Lippert H. Oncological outcome of local vs radical resection of low-risk pT1 rectal cancer. Arch Surg 2007;142(7): 649-655i discussion 656 .

Bentrem DJ, Okabe S, Wong WD, Guillem JG, Weiser MR, Temple LK, Ben-Porat LS, Minsky BD, Cohen AM, Paty PB. T1 adenocarcinoma of the rectum: transanal excision or radical surgery? Ann Surg 2005;242(4): 472-477; discussion 477-479.

O'Neill BD, Brown G, Heald RJ, Cunningham D, Tait DM. Non-operative treatment after neoadjuvant chemoradiotherapy for rectal cancer. Lancet Oncol 2007;8(7): 625-633.

Lezoche G, Baldarelli M, Guerrieri M, Paganini AM, De Sanctis A, Bartolacci S, Lezoche E. A prospective randomized study with a 5-year minimum follow-up evaluation of transanal endoscopic microsurgery versus laparoscopic total mesorectal excision after neoadjuvant therapy. Surg Endosc 2008;22(2): 352-358.

Allen SD, Padhani AR, Dzik-Jurasz AS, Glynne-Jones R. Rectal carcinoma: MRI with histologic correlation before and after chemoradiation therapy. AJR Am J Roentgenol 2007;188(2): 442-451. Chen CC, Lee RC, Lin JK, Wang LW, Yang SH. How accurate is magnetic resonance imaging in restaging rectal cancer in patients receiving preoperative combined chemoradiotherapy? Dis Colon Rectum 2005;48(4): 722-728.

Kuo LJ, Chern MC, Tsou MH, Liu MC, Jian JJ, Chen CM, Chung YL, Fang WT. Interpretation of magnetic resonance imaging for locally advanced rectal carcinoma after preoperative chemoradiation therapy. Dis Colon Rectum 2005;48(1): 23 -28.

Will O, Purkayastha S, Chan C, Athanasiou T, Darzi AW, Gedroyc W, Tekkis PP. Diagnostic precision of nanoparticle-enhanced MRI for lymph-node metastases: a meta-analysis. Lancet Oncol $2006 ; 7(1): 52-60$. 
18. Lahaye MJ, Engelen SM, Beets $G$, de Bruine $A$, von Meyenfeldt $M$, van Engelshoven JM, van de Velde $\mathrm{CH}$, Beets-Tan R. How accurate can we predict the nodal status in primary rectal cancer with USPIO MRI? Ann Oncol 2006;17 Suppl 1: i11

19. Weissleder $R$, Elizondo $G$, Wittenberg J, Rabito $C A$, Bengele $H H$, Josephson L. Ultrasmall superparamagnetic iron oxide: characterization of a new class of contrast agents for MR imaging. Radiology 1990;175(2): 489-493.

20. Lahaye $M J$, Engelen $S M$, Kessels $A G$, de Bruine AP, von Meyenfeldt MF, van Engelshoven JM, van de Velde CJ, Beets GL, Beets-Tan RG. USPIO-enhanced MR imaging for nodal staging in patients with primary rectal cancer: predictive criteria. Radiology 2008;246(3): 804-811.

21. Quirke $P$, Dixon MF. The prediction of local recurrence in rectal adenocarcinoma by histopathological examination. Int J Colorectal Dis 1988;3(2): 127-131.

22. DeLong ER, DeLong DM, Clarke-Pearson DL. Comparing the areas under two or more correlated receiver operating characteristic curves: a nonparametric approach. Biometrics 1988;44(3): $837-845$. Suppiah A, Hunter IA, Cowley J, Garimella V, Cast J, Hartley JE, Monson JR. Magnetic resonance imaging accuracy in assessing tumor downstaging following chemoradiation in rectal cancer. Colorectal Dis 2008; [E-pub ahead of print].

24. Kulkarni T, Gollins S, Maw A, Hobson P, Byrne R, Widdowson D. Magnetic resonance imaging in rectal cancer downstaged using neoadjuvant chemoradiation: accuracy of prediction of tumor stage and circumferential resection margin status. Colorectal Dis 2008;10(5): 479-489.

25. Vanagunas $A$, Lin DE, Stryker SJ. Accuracy of endoscopic ultrasound for restaging rectal cancer following neoadjuvant chemoradiation therapy. Am J Gastroenterol 2004;99(1): 109-112.

26. Liersch T, Langer C, Jakob C, Muller D, Ghadimi BM, Siemer A, Markus PM, Fuzesi L, Becker H. [Preoperative diagnostic procedures in locally advanced rectal carcinoma ( $>$ or $=T_{3}$ or $N+$ ). What does endoluminal ultrasound achieve at staging and restaging (after neoadjuvant radiochemotherapy) in contrast to computed tomography?]. Chirurg 2003;74(3): 224-234.

27. Dresen RC, Beets GL, Rutten $H$, Engelen SM, Lahaye MJ, Vliegen RF, De Bruine A, Kessels $A G$, Lammering $G$, Beets-Tan RG. MRI for restaging after neoadjuvant radiochemotherapy in locally advanced rectal cancer. Part I: Are we able to predict tumor confined to the rectal wall? Radiology 2008;in press

28. Wang $C$, Zhou Z, Wang Z, Zheng $Y$, Zhao G, Yu Y, Cheng Z, Chen D, Liu W. Patterns of neoplastic foci and lymph node micrometastasis within the mesorectum. Langenbecks Arch 5urg 2005;390(4): 312-318.

Koh D-M, Brown G, Temple L, Raja A, Toomey P, Bett N, Norman AR, Husband JE. Rectal Cancer: Mesorectal Lymph Nodes at MR Imaging with USPIO versus Histopathologic Findings--Initial Observations. Radiology 2004;231(1): 91-99.

30. Habr-Gama A, Perez RO, Proscurshim I, Campos FG, Nadalin W, Kiss D, Gama-Rodrigues J. Patterns of failure and survival for nonoperative treatment of stage co distal rectal cancer following neoadjuvant chemoradiation therapy. J Gastrointest Surg 2006;10(10): 1319-1328; discussion 13281319 .

31. Vliegen RF, Beets $G L$, Lammering $G$, Dresen RC, Rutten HJ, Kessels AG, Oei TK, de Bruine AP, van Engelshoven JM, Beets-Tan RG. Mesorectal fascia invasion after neoadjuvant chemotherapy and radiation therapy for locally advanced rectal cancer: accuracy of MR imaging for prediction. Radiology 2008;246(2): 454-462.

Guillem JG, Diaz-Gonzalez JA, Minsky BD, Valentini V, Jeong SY, Rodriguez-Bigas MA, Coco C, Leon R, Hernandez-Lizoain JL, Aristu JJ, Riedel ER, Nitti D, Wong WD, Pucciarelli S. cT3No rectal cancer: potential overtreatment with preoperative chemoradiotherapy is warranted. $J$ Clin Oncol 2008;26(3): 368-373. Bujko K, Sopylo R, Kepka L. Local excision after radio(chemo)therapy for rectal cancer: is it safe? Clin Oncol (R Coll Radiol) 2007;19(9): 693-700.

Lahaye MJ, Engelen SM, Beets GL, Kessels AG, Post PJ, Dohmen JP, Opdenakker GR, Van de Velde CJ, Van Engelshoven JM, Beets-Tan RG. Accuracy of USPIO-enhanced MRI for local staging of rectal cancer: a Multicenter Study in expert and three regional centers. . Ann Oncol 2008;19 (suppl 1): i33. 
Summary, discussion and conclusions 


\section{Chapter 9}

In 1935, F. Yeomans ${ }^{1}$ stated: "Radical surgery for rectal cancer has now attained such a degree of technical perfection that it would seem that slight, if any, advance in operative technique can be looked for." In reality, not only surgical technique has been subject to major changes since then, but also (neo)adjuvant treatment regimens. And even at present, defining the optimal treatment for rectal cancer patients still is an ongoing process that has to adapt to evolving medical technologies and knowledge. Of the significant changes in rectal cancer treatment in the past decades, the most important probably is the introduction of Total Mesorectal Excision (TME) surgery in the early $1980^{\prime} \mathrm{s}^{2}$. At the same time, it was recognized through randomized trials that there is an important role for preoperative radiotherapy. The Swedish Rectal Cancer Trial (SRCT) and the Dutch TME trial showed an advantage of a short course preoperative radiotherapy for all patients ${ }^{3 / 4}$. It was recognized that a short course preoperative radiation is not required for stage I ( $\left.\mathrm{T}_{1-2 \mathrm{No}}\right)$ tumors who have an excellent prognosis without radiotherapy ${ }^{4}$. At the other end of the spectrum are locally advanced tumors that invade or come close to the mesorectal fascia. The short

142 course of preoperative radiotherapy does not compensate for an involved circumferential resection margin, i.e. the distance of the tumor to the mesorectal fascia, which is the plane of resection in TME surgery ${ }^{5}$. Therefore a more extensive neoadjuvant treatment is required for these more advanced tumors. A long course of preoperative chemoradiotherapy has been shown to be beneficial in several randomized studies ${ }^{6-9}$. The third major change was the improvement in imaging, that allowed a better preoperative identification of risk factors for local recurrence. Chapter 2 of this thesis gives an overview of the available techniques in 2007, with an emphasis on the developments in MR ${ }^{10}$. MRI can accurately anticipate an involved CRM and therefore identify locally advanced tumors ${ }^{12-15}$. Furthermore, with the use of contrast agents, MRI is becoming increasingly more capable of accurate prediction of nodal status, another important risk factor for local recurrence ${ }^{16,17}$. With a good preoperative prediction of the risk factors for local recurrence patients can be stratified in low, intermediate and high risk groups for local recurrence, and can be treated accordingly, as described below. 
The primary aim of this thesis was to evaluate whether the use of preoperative $M R I$ as a selection tool for the different risk groups of rectal cancer patients and the subsequent differentiated treatment leads to an improved surgical outcome. Chapter 6 describes the results of a prospective multicenter cohort of rectal cancer patients. Patients were stratified into different treatment groups according to their risk for local recurrence, based on MR imaging. The low risk group was defined as T1-2No tumors, or mid and high rectal $T_{3}$ tumors with a wide lateral margin, and the high risk group was defined as $T_{3}$ with threatened/involved mesorectal fascia or $\mathrm{T}_{4}$ and/or massive nodal involvement and/or very distal $T_{3}$ tumors. The other tumors were classified as intermediate risk'. The general strategy was to treat the low risk group with surgery only, the intermediate risk group with $5 \times 5$ Gy radiotherapy and surgery, and the high risk group with chemoradiotherapy and surgery. The study shows that this differentiated treatment led to an improved number of complete resections (95.6\%) as compared to a historical control (84\%) when no standardized preoperative imaging was used ${ }^{4}$. There is one other multicenter cohort study in which preoperative MR imaging in rectal cancer was systematically used to guide differentiated treatment, and a Ro resection rate of $87 \%$ was reported ${ }^{28}$. In the present study, the 2-year local recurrence rate of $2.8 \%$ is low, as compared to other studies, although a longer follow-up is needed to establish the long term recurrence and survival rates.

In the low risk group of early tumors a local excision was performed in $43 \%$ of patients, instead of TME surgery. In this approach it is important to exclude node positive tumors, because the majority of recurrences after TEM are thought to be caused by unrecognized nodal involvement ${ }^{19}$. With the use of a lymph node specific MR contrast agent with a high negative predictive value (chapter 5 ), it is expected that the risk for recurrence is low. There are several reports on the use of local excisions in early rectal cancer ${ }^{19-26}$. Because of the inability to detect nodal disease it is only recommended in low grade $\mathrm{pT} T_{1}$ rectal cancer, because of the very low prevalence of nodal metastases ${ }^{21,25}$. For unfavorable $p T_{1}$ tumors as well as pT2 tumors the risk for local recurrence is too high ${ }^{21,25}$. Neoadjuvant chemoradiation has been proposed to prevent this ${ }^{21,24}$. Another approach could be to improve nodal staging, as we did in our study, to increase the likelihood of selecting only true node negative tumors for a local excision. More studies 
Chapter 9

incorporating improved nodal staging are required before definitive conclusions can be drawn.

As a secondary aim this thesis evaluated the accuracy of MRI and a lymph node specific contrast agent for the prediction of T stage and nodal disease. Chapter 4 describes the development of criteria for evaluating lymph nodes with USPIO $M R{ }^{27}$. The most practical predictive criterion for nodal prediction with USPIO MRI is the estimated percentage of white region within a node: With a cutoff point of more than $30 \%$ white region the sensitivity and specificity for prediction of a malignant node is 0.93 and 0.96 respectively. Chapter 5 reports on the T-stage and nodal prediction on a patient basis in a prospective multicenter setting. The results of the study show that a tumor limited to the bowel wall can be predicted with a low risk of understaging of the tumor. This means that when a radiologist states the tumor is limited to the bowel wall, he is correct in majority of cases $(95 \%)$. With regard to $\mathrm{N}$-stage, USPIO MRI is useful in multicenter setting for the prediction of No tumors, again with a low risk of understaging (NPV=97\%). However, the PPV of USPIO MRI is a little lower (70\%), which has led to a considerable overstaging of stage I rectal cancer patients, which were staged as node positive while they were in fact node negative. However, when the radiologist predicts a tumor to be stage $\mathrm{I}$, he is correct in majority of cases $(0.94$ and 0.92 for expert and general radiologist respectively).

Dedicated lymph node MR imaging offered the opportunity to study the location of the malignant lymph nodes, which may help the radiologist to predict and the surgeon to find them. Chapter 3 shows that the majority of positive lymph nodes are located in the posterior part of the mesorectum, at tumor height or above ${ }^{28}$.

The outcome of multimodality treatment of locally advanced rectal cancer is described in chapter 7 . The retrospective study shows that with an aggressive multimodal approach, a good local control of around $90 \%$ at 5 years is obtained, although at the expense of considerable morbidity: $30 \%$ of patients suffered from major complications. Postoperative mortality in the first 30 days was $2.6 \%$. An important observation of this study is that while local disease is well controlled, the risk for distant metastases remains high, leading to a 5 year overall survival of $65 \%$. There are several options to address the risk of distant metastases in locally 
advanced rectal cancer. The first is to improve adjuvant chemotherapy regimens, including biological agents. A second approach is to give the chemotherapy in a neoadjuvant rather than an adjuvant setting ${ }^{29}$. This would avoid the problem that many patients do not receive their postoperative chemotherapy as a result of serious complications of surgery. Another issue that needs to be addressed is the optimal timing of surgical resection after neoadjuvant chemoradiation therapy. Some studies indicate that a longer waiting time between radiotherapy and surgery does not lead to a better response rate ${ }^{30}$, whereas others state that a waiting time $>7$ weeks leads to more pathological complete responses and a better disease-free survival ${ }^{31}$. Furthermore, a recent study showed that a longer waiting time leads to less perioperative morbidity, without compromising outcome $^{32}$. More (randomized) trials are needed for solving these discrepancies in literature.

With regard to patients who not only present with locally advanced disease, but also with (irresectable) distant metastases, mostly liver metastases, the optimal treatment is still to be defined. There is evidence that high dose chemotherapy, followed by a liver resection, and finally a resection of the primary tumor might be a good approach ${ }^{33}$, but further clinical research is needed to draw definitive conclusions ${ }^{34}$.

Chapter $\mathbf{8}$ of this thesis focused on the identification of good responders to chemoradiotherapy for locally advanced disease. An accurate identification could lead to the possibility of further differentiation to a more conservative surgical treatment (TEM) in the good responders to CRT. TEM is at the moment still a controversial option after chemoradiotherapy, leading to less oncological control because the mesorectum with its lymph nodes is left in situ, however it has the major advantage of minimal morbidity ${ }^{35}$. In the prospective multicenter study in this chapter postchemoradiation MRI was assessed with regard to the ability to predict $\mathrm{T}$ and $\mathrm{N}$ stage. The results show that for prediction of tumors that do not penetrate the bowel wall anymore ( $\mathrm{T}_{1-2}$ ) and tumors that have become nodenegative (No), MRI again has a low risk of understaging (NPV of 0.90 and 0.95 respectively). As with prechemoradiation prediction however, there is a considerable risk of overstaging with a PPV of 0.64 for prediction of a tumor that still penetrates the bowel wall and also a PPV of 0.64 for prediction of a positive 


\section{Chapter 9}

node. If we combine prediction of $T$ and $N$ to identify stage I disease, there is a high predictive value of the MR identification of stage 1, 0.92, however again at the expense of considerable overstaging. So when the radiologist concludes that the tumor has become limited to the bowel wall, and that there are no involved lymph nodes, the clinician can consider the option of a local excision instead of major surgery. In the present study we only included locally advanced tumors, but the use of CRT + local excision can also be further explored in less advanced cases, were CRT might lead to even more cases suitable for local excision. This treatment regimen has been shown to lead to good results by Lezoche et al., in a small randomized study which compared CRT followed by TEM vs. CRT followed by laparoscopic $T M E^{22}$. One could even go one step further, even more controversial, and propose a wait-and-see policy in selected patients with a complete response to CRT. One such study, by Habr Gama et al. shows encouraging results ${ }^{36}$. For both controversial less invasive options more prospective, preferably randomized, studies are needed. For the radiologist the challenge is to predict a pathological complete response to enable such strategy. Furthermore, the role of MRI as a follow up tool to detect recurrences in an early stage requires exploration.

The results of this project show that overall there was some difference in performance for $(y) T$ and $(y) N$ stage prediction at 1.0 Tesla compared to 1.5 Tesla. Although the total number of patients enrolled at both 1 Tesla centers were disproportionately lower (chapter 4) and too limited (chapter 8) as compared to that enrolled at both 1.5 Tesla centers and certainly definite conclusions cannot be drawn from the data of this project, our studies suggest that $(y) T$ and $(y) N$ stage assessment in rectal cancer staging at 1.0 Tesla MR should not be relied on heavily and is therefore less recommended, while this was not the case with CRM assessment. The inherent inferior SNR of low field strength 1Tesla MR machines does not allow pixel volumes as small as with 1.5 T. Balancing between the best possible contrast and best possible spatial resolution, we cannot but compromise on the pixel sizes, in order not to lose contrast. As a consequence the pixel volumes with 1 Tesla MRIs for the $2 D T_{2} W, 3 D T_{1} W$ and $3 D$ $T_{2} * W$ sequences are 7,3 and 3.7 times larger compared to the 1.5 Tesla MRI. This could well be related to the worse performance of the less experienced reader on 
Summary, Discussion and Conclusions

1.0T than an experienced reader, especially for $y^{T}$ staging. Possibly the more expert reader takes into account other factors when assessing risk factors, such as the information from the initial staging MRI on tumor size, initial $\mathrm{T}$ and $\mathrm{N}$ staging as well as fraction of volume reduction. This could explain that there is less difficulty in assessing a y To-2 tumor (and $y \mathrm{No}$ ) on a restaging MRI on 1.0T.

\section{Future prospects}

The approach in rectal cancer treatment has shifted from one standard treatment for the majority of rectal cancer patients to choosing from different options the one that provides the best outcome with the least morbidity for an individual patient. These differentiated treatment options are based on previous scientific work as well as some of the results of the studies described in this thesis. Unlike Yeomans in $1935^{1}$, at this moment we do not believe current rectal cancer treatment has reached its end stage with regard to possibilities, as indicated by the proposals for ongoing research mentioned above. This will certainly lead to further improvement in surgical techniques, chemoradiotherapy regimens, targeted therapy, nuclear and MR molecular imaging and contrast agents, and will without a doubt lead to further developments in the treatment, and the outcome of rectal cancer patients.

\section{CONCLUSIONS}

* The use of preoperative MRI as a selection tool for the different risk groups of rectal cancer patients and the subsequent differentiated treatment leads to an improved number of complete resections as compared to a historical control, with encouraging low local recurrence rates.

Preoperative MRI with a lymph node specific contrast agent in primary rectal cancer can select tumors limited to the bowel wall and tumors with a negative nodal status with high negative predictive value in expert as well as general setting. This enables selection of early tumors (stage I), who do not require neoadjuvant radiotherapy. 


\section{Chapter 9}

- Multimodality treatment of locally advanced rectal cancer leads to a good local control of $90 \%$ at 5 years, however at the expense of considerable morbidity. Distant recurrence free and overall survival remain however troublesome and future research should be focusing on prevention, detection and treatment of distant metastases.

- MRI after CRT can select tumors that do not penetrate the bowel wall anymore as well as tumors that have become node negative with high negative predictive value, in expert as well as general setting. Therefore, future research can focus on safe selection of those tumors that could be treated with a local excision instead of a standard surgical resection. 


\section{REFERENCES}

1. Yeomans FC. Two Stage Operation for Rectal Cancer. Ann Surg 1935;102(1): 68-72.

2. Heald RJ, Ryall RD. Recurrence and survival after total mesorectal excision for rectal cancer. Lancet 1986;1(8496): 1479-1482.

3. Improved survival with preoperative radiotherapy in resectable rectal cancer. Swedish Rectal Cancer Trial. N Engl J Med 1997;336(14): 980-987.

4. Kapiteijn E, Marijnen CA, Nagtegaal ID, Putter $H$, Steup WH, Wiggers $T$, Rutten $H J$, Pahlman $L$, Glimelius $B$, van Krieken $\mathrm{JH}$, Leer JW, van de Velde CJ. Preoperative radiotherapy combined with total mesorectal excision for resectable rectal cancer. N Engl J Med 2001;345(9): 638-646.

5. Marijnen CA, Nagtegaal ID, Kapiteijn E, Kranenbarg EK, Noordijk EM, van Krieken JH, van de Velde $\mathrm{CJ}$, Leer JW. Radiotherapy does not compensate for positive resection margins in rectal cancer patients: report of a multicenter randomized trial. Int J Radiat Oncol Biol Phys 2003;55(5): 1311-1320.

6. Saver R, Becker H, Hohenberger W, Rodel C, Wittekind C, Fietkau R, Martus P, Tschmelitsch J, Hager E, Hess CF, Karstens JH, Liersch T, Schmidberger $H$, Raab R. Preoperative versus postoperative chemoradiotherapy for rectal cancer. N Engl J Med 2004;351(17): 1731-1740.

7. Bosset JF, Collette L, Calais G, Mineur L, Maingon P, Radosevic-Jelic L, Daban A, Bardet $E$, Beny A, Ollier JC. Chemotherapy with preoperative radiotherapy in rectal cancer. N Engl J Med 2006;355(11): $1114-1123$.

8. Braendengen $M$, Tveit KM, Berglund $A$, Birkemeyer $E$, Frykholm $G$, Pahlman $L$, Wiig JN, Bystrom $P$, Bujko $K$, Glimelius B. Randomized phase III study comparing preoperative radiotherapy with chemoradiotherapy in nonresectable rectal cancer. J Clin Oncol 2008;26(22):3687-3694.

9. Gerard JP, Conroy T, Bonnetain F, Bouche $O$, Chapet $O$, Closon-Dejardin MT, Untereiner M, Leduc B, Francois E, Maurel J, Seitz JF, Buecher B, Mackiewicz R, Ducreux M, Bedenne L. Preoperative radiotherapy with or without concurrent fluorouracil and leucovorin in $T_{3-4}$ rectal cancers: results of FFCD 9203. J Clin Oncol 2006;24(28): 4620-4625.

10. Engelen SM, Beets GL, Beets-Tan RG. Role of preoperative local and distant staging in rectal cancer. Onkologie 2007;30(3): 141-145.

11. Beets-Tan RG, Beets GL, Vliegen RF, Kessels AG, Van Boven $H$, De Bruine A, von Meyenfeldt MF, Baeten $C G$, van Engelshoven JM. Accuracy of magnetic resonance imaging in prediction of tumorfree resection margin in rectal cancer surgery. Lancet 2001;357(9255): 497-504.

12. Bissett IP, Fernando CC, Hough DM, Cowan BR, Chau KY, Young AA, Parry BR, Hill GL. Identification of the fascia propria by magnetic resonance imaging and its relevance to preoperative assessment of rectal cancer. Dis Colon Rectum 2001;44(2): 259-265.

13. Blomqvist L, Rubio C, Holm T, Machado M, Hindmarsh T. Rectal adenocarcinoma: assessment of tumor involvement of the lateral resection margin by MRI of resected specimen. $\mathrm{Br} J$ Radiol 1999;72(853): 18-23.

14. Brown G, Richards CJ, Newcombe RG, Dallimore NS, Radcliffe AG, Carey DP, Bourne MW, Williams GT. Rectal Carcinoma: Thin-Section MR Imaging for Staging in 28 Patients. Radiology 1999;211(1): 215-222.

15. Lahaye MJ, Engelen SM, Nelemans PJ, Beets GL, van de Velde CJ, van Engelshoven JM, Beets-Tan RG. Imaging for predicting the risk factors--the circumferential resection margin and nodal disease-of local recurrence in rectal cancer: a meta-analysis. Semin Ultrasound CTMR 2005;26(4): 259-268.

16. Will O, Purkayastha S, Chan C, Athanasiou T, Darzi AW, Gedroyc W, Tekkis PP. Diagnostic precision of nanoparticle-enhanced MRI for lymph-node metastases: a meta-analysis. Lancet Oncol 2006; $7(1)$ : 52-60.

17. Lahaye MJ, Engelen SM, Beets GL, de Bruine AP, von Meyenfeldt MF, Van Engelshoven JM, Van de Velde CJ, Beets-Tan RG. How accurate can we predict the nodal status in primary rectal cancer with USPIO MRI? Ann Oncol 2006;17 (suppl): i11-12.

18. Salerno G, Daniels IR, Moran BJ, Wotherspoon A, Brown G. Clarifying margins in the multidisciplinary management of rectal cancer: the MERCURY experience. Clin Radio/ 2006;61(11): 916-923.

19. Tytherleigh MG, Warren BF, Mortensen NJ. Management of early rectal cancer. Br J Surg 2008;95(4): 409-423. 


\section{Chapter 9}

20. Baatrup $G$, Breum B, Qvist $N$, Elbrond $H$, Moller P, Hesselfeldt P. Transanal endoscopic microsurgery in 143 consecutive patients with rectal adenocarcinoma. Results from a Danish multicenter study. Colorectal Dis 2008.

21. Suppiah A, Maslekar S, Alabi A, Hartley JE, Monson JR. Transanal endoscopic microsurgery in early rectal cancer: time for a trial? Colorectal Dis 2008;10(4):314-327; discussion 327-319.

22. Lezoche $G$, Baldarelli $M$, Guerrieri $M$, Paganini AM, De Sanctis A, Bartolacci S, Lezoche E. A prospective randomized study with a 5 -year minimum follow-up evaluation of transanal endoscopic microsurgery versus laparoscopic total mesorectal excision after neoadjuvant therapy. Surg Endosc 2008;22(2): 352-358.

23. Ptok $H$, Marusch F, Meyer F, Schubert D, Koeckerling F, Gastinger I, Lippert H. Oncological outcome of local vs radical resection of low-risk pT1 rectal cancer. Arch Surg 2007;142(7): 649-655; discussion 656.

24. Borschitz $T$, Wachtlin $D$, Mohler $M$, Schmidberger $H_{1}$ Junginger T. Neoadjuvant chemoradiation and local excision for T2-3 rectal cancer. Ann Surg Oncol 2008;15(3): 712-720.

25. Borschitz T, Gockel I, Kiesslich $R$, Junginger $T$. Oncological outcome after local excision of rectal carcinomas. Ann Surg Oncol 2008;15(11): 3101-3108.

26. Balch GC, De Meo A, Guillem JG. Modern management of rectal cancer: a 2006 update. World $J$ Gastroenterol 2006;12(20): 3186-3195.

27. Lahaye MJ, Engelen SM, Beets GL, Kessels AG, Post PJ, Dohmen JP, Opdenakker GR, Van de Velde CJ, Van Engelshoven JM, Beets-Tan RG. Accuracy of USPIO-enhanced MRI for local staging of rectal cancer: a Multicenter Study in expert and three regional centers. . Ann Oncol 2008;19 (suppl 1): i33.

28. Engelen SM, Beets-Tan RG, Lahaye MJ, Kessels AG, Beets GL. Location of involved mesorectal and extramesorectal lymph nodes in patients with primary rectal cancer: preoperative assessment with MR imaging. Eur J Surg Oncol 2008;34(7): 776-781.

29. Chau I, Brown G, Cunningham D, Tait D, Wotherspoon A, Norman AR, Tebbutt $N$, Hill $M$, Ross $P J$, Massey A, Oates J. Neoadjuvant capecitabine and oxaliplatin followed by synchronous chemoradiation and total mesorectal excision in magnetic resonance imaging-defined poor-risk rectal cancer. J Clin Oncol 2006;24(4): 668-674.

30. Lim SB, Choi HS, Jeong SY, Kim DY, Jung KH, Hong YS, Chang HJ, Park JG. Optimal surgery time after preoperative chemoradiotherapy for locally advanced rectal cancer5. Ann 5 urg 2008;248(2): 243-251.

31. Tulchinsky H, Shmueli E, Figer A, Klausner JM, Rabau M. An interval >7 weeks between neoadjuvant therapy and surgery improves pathologic complete response and disease-free survival in patients with locally advanced rectal cancer. Ann Surg Oncol 2008;15(10): 2661-2667.

32. Kerr SF, Norton S, Glynne-Jones R. Delaying surgery after neoadjuvant chemoradiotherapy for rectal cancer may reduce postoperative morbidity without compromising prognosis. Br J Surg 2008;95(12): $1534-1540$.

33. Mentha G, Majno PE, Andres A, Rubbia-Brandt L, Morel P, Roth AD. Neoadjuvant chemotherapy and resection of advanced synchronous liver metastases before treatment of the colorectal primary. $B r \mathrm{~J}$ Surg 2006;93(7): 872-878.

34. Pozzo $C_{1}$ Barone $C_{1}$ Kemeny NE. Advances in neoadjuvant therapy for colorectal cancer with liver metastases. Cancer Treat Rev 2008;34(4): 293-301.

35. Caricato $M$, Borzomati D, Ausania F, Tonini G, Rabitti C, Valeri S, Trodella L, Ripetti V, Coppola R Complementary use of local excision and transanal endoscopic microsurgery for rectal cancer after neoadjuvant chemoradiation. Surg Endosc 2006;20(8): 1203-1207.

36. Habr-Gama A, Perez RO, Proscurshim I, Campos FG, Nadalin W, Kiss D, Gama-Rodrigues J. Patterns of failure and survival for nonoperative treatment of stage co distal rectal cancer following neoadjuvant chemoradiation therapy. J Gastrointest Surg 2006;10(10): 1319-1328; discussion 13281319. 
Samenvatting 


\section{Chapter 10}

Eén-derde van de gevallen van kanker van de dikke darm is gelokaliseerd in de endeldarm. Een tumor in de endeldarm noemt men een rectumcarcinoom. Dit rectumcarcinoom is niet alleen berucht om het kankerspecifiek overlijden, maar ook om de kans dat de tumor na chirurgische behandeling terugkomt; een lokaal recidief. In de afgelopen decaden zijn er verschillende veranderingen geweest met betrekking tot de behandeling van het rectumcarcinoom. Op chirurgisch vlak is de belangrijkste verandering de ontwikkeling van de Totale Mesorectal Excisie (TME), een chirurgische techniek waarbij de endeldarm samen met het omliggende vet -waarin lymfklieren en bloedvaten liggen, via welke de kanker kan uitbreiden naar de omgeving- wordt verwijderd. De darm en dit omliggende vet wordt omgeven door de mesorectale fascie, een vlies dat derhalve wordt aangehouden als het vlak waarlangs de chirurg opereert; het resectievlak. Met de invoering van de TME is er een vermindering van het aantal lokaal recidieven bewerkstelligd. Naast deze chirurgische verandering, heeft zich ook een verandering voorgedaan in de aanvullende therapieën zoals radiotherapie (bestraling) en chemotherapie bij het rectumcarcinoom. Allereerst is aangetoond dat het toedienen van een korte kuur bestralingen ( $5 \times 5$ Gy) vóór de operatie het risico op een lokaal recidief verminderd. Dit is aangetoond in zowel de Swedish Rectal Cancer Trial, waar geen gestandaardiseerde chirurgie werd gebruikt, als in de Dutch TME trial, waar wel TME chirurgie werd gebruikt. Uit subgroepanalyses van de studies die dit aantonen blijkt echter ook dat een bepaalde groep tumoren, de vroege tumoren zonder invasie van de lymfklieren, deze korte kuur bestralingen niet nodig is, omdat bij deze patiënten de kans op een lokaal recidief toch al klein is. Verder blijkt dat bij uitgebreide tumoren, die reiken tot dicht bij de mesorectale fascie of daar zelfs doorheen groeien, een korte kuur radiotherapie niet genoeg is. $\mathrm{Bij}$ deze patiënten is een meer uitgebreide therapie nodig, omdat het risico te groot is dat de chirurg tumor achterlaat, wat leidt tot hernieuwde tumorgroei. Uit onderzoek blijkt dat bij deze patiënten een lange kuur radiotherapie in combinatie met chemotherapie de kans op een lokaal recidief wel verminderd. Het beste kan ook deze kuur preoperatief gegeven worden.

Een andere belangrijke ontwikkeling in de behandeling van het rectumcarcinoom is de verbeterde preoperatieve beeldvorming, die ervoor zorgt dat de patiënten 
met een uitgebreide tumor beter geselecteerd kunnen worden. Hoofdstuk 2 van dit proefschrift geeft een overzicht van de beschikbare methoden van beeldvorming, en richt zich met name op de ontwikkeling van de beeldvorming met MRI. MRI kan zeer nauwkeurig de afstand schatten van de tumor tot aan de mesorectale fascie, dus tot aan het resectievlak van de chirurg. Derhalve kan MRI de lokaal uitgebreide tumoren accuraat identificeren. MRI is verder in staat een andere belangrijke risicofactor voor lokaal recidief te voorspellen: de lymfklierstatus. Als deze risicofactoren voor lokaal recidief goed kunnen worden ingeschat, is het mogelijk om tumoren in te delen in verschillende behandelingsgroepen op basis van het risico op lokaal recidief.

Het primaire doel van dit proefschrift was om te evalueren of een op maat gemaakt behandeling van het rectumcarcinoom op basis van een risicoinschatting voor recidief met preoperatieve beeldvorming middels MRI leidt tot een groter percentage tumoren die compleet verwijderd worden, in vergelijking met een historische controle, de Dutch TME trial, waar geen gestandaardiseerde preoperatieve beeldvorming werd gebruikt. Het verbeteren van het aantal complete resecties zou op de lange termijn moeten leiden tot een lager lokaal recidief percentage. Dit primaire doel van dit proefschrift wordt beschreven in hoofdstuk 6. Dit hoofdstuk beschrijft de resultaten van een prospectieve multicenter cohort studie van rectumcarcinoompatiënten. De patiënten werden op basis van preoperatieve MRI ingedeeld in verschillende behandelingsgroepen gebaseerd op het geschatte risico op lokaal recidief. De laag-risico groep werd gedefinieerd als T1-2No tumoren (tumoren die maximaal tot in de spierlaag van de darmwand groeien, maar niet daar door heen, zonder positieve lymfklieren) of T3 tumoren (wel doorgroei door de spierlaag heen) die mid- of hoogrectaal gelegen zijn, met een wijde marge tot aan de mesorectale fascie. De hoog-risico groep werd gedefinieerd als tumoren die tot aan of doorheen de mesorectale fascie groeiden, of zelf in omliggende organen ( $T_{4}$ tumor) en/of veel positieve klieren hadden en/of tenminste $T_{3}$ tumor waren en zeer laag in de endeldarm lagen. Alle andere tumoren werden gedefinieerd als 'intermediate risk'. 'Laagrisico tumoren' werden behandeld met enkel chirurgische resectie, zonder voorafgaande (neo-adjuvante) radio- of chemotherapie. 'Intermediate- risk tumoren' werden behandeld met een korte kuur bestralingen ( $5 \times 5$ Gy) gevolgd 


\section{Chapter 10}

door TME, en 'hoog- risico tumoren' werden behandeld met een lange kuur neoadjuvante radiochemotherapie, gevolgd door een vaak uitgebreidere resectie. Deze studie toont aan dat het aantal complete resecties stijgt tot $95.6 \%$ in vergelijking met de historische controle $(84 \%)$, de Dutch TME trial. Het percentage lokaal recidieven na 2 jaar is $2,8 \%$, hetgeen laag is, maar een langere follow-up van de patiënten is nodig om definitieve uitspraken te doen over het gunstig effect op het lokaal recidief percentage.

Een secundair doel van dit proefschrift was het evalueren van de accuratesse van MRI voor het voorspellen van tumorstadium (T-stage) en klierstadium ( $N$-stage), voor dit laatste geholpen door een lymfklierspecifiek contrastmiddel, USPIO (Ultrasmall Paramagnetic Iron Oxide). Hoofdstuk 4 van dit proefschrift beschrijft de ontwikkeling van criteria op basis waarvan een USPIO MRI beoordeeld kan worden op positieve of negatieve klieren. Het meest praktische criterium voor het voorspellen of een klier positief of negatief is, blijkt het geschatte percentage wit van de klier te zijn: als een klier voor meer dan $30 \%$ wit is, is de sensitiviteit en specificiteit voor het voorspellen van een tumoraal geïnvadeerde klier respectievelijk 0.93 en 0.96 . Anders gezegd: een klier die overwegend zwart is, is waarschijnlijk negatief ('schoon'), terwijl een klier die voor meer dan $30 \%$ wit is, waarschijnlijk tumorcellen bevat.

Hoofdstuk 5 laat zien hoe goed het T-stadium en het klierstadium voorspeld kan worden in een multicenter studie, dus zowel door een expert op het gebied van MRI van het kleine bekken, als ook door de 'gewone' radioloog, die niet specifiek gespecialiseerd is in dit gebied. De resultaten van de studie laten zien, dat een tumor die beperkt blijft tot de rectumwand, dus niet door de darm heengroeit in het omliggende vetweefsel, met grote zekerheid kan worden voorspeld (95\%), zowel door een expert, als een gewone radioloog. Met betrekking tot het klierstadium, kan de radioloog met grote zekerheid aangeven of een klier niet tumoraal geinvadeerd is (negatief voorspellende waarde $(N P V)=0.97$ ). Deze hoge NPV brengt wel een lagere positief voorspellende waarde, PPV, met zich mee, dat wil zeggen dat de radioloog een aantal klieren foutief als wel tumoraal geïnvadeerd afgeeft. Dit betekent echter dat als een radioloog zegt dat een tumor een laag risico tumor is, dit in de meerderheid van de gevallen correct is, 
en het dus veilig is om deze patiënten direct te opereren zonder eerst te bestralen.

Het gebruik van MRI met een lymfklierspecifiek contrastmiddel gaf tevens de mogelijkheid om de de lokatie van maligne lymfklieren te bestuderen, hetgeen de radioloog kan helpen om de klieren te voorspellen en de chirurg om ze te vinden. Hoofdstuk 3 laat zien dat het merendeel van de maligne lymfklieren gesitueerd is in het posterieure deel van het mesorectum, ter hoogte van de tumor of daarboven.

In hoofdstuk 7 van dit proefschrift wordt dieper ingegaan op de prognose van patiënten met een lokaal uitgebreid rectumcarcinoom. In de retrospectieve studie die hier wordt beschreven, zien we een goed lokale controle ( $90 \%$ recidiefvrij bij 5 jaar follow-up) van patiënten met een lokaal uitgebreide tumor die agressief behandeld worden in multidisciplinair verband (radiotherapie, chemotherapie, vitgebreide chirurgie). Voor deze goede lokale controle wordt echter wel een prijs betaald: $30 \%$ van de patiënten had grote complicaties door de agressieve behandeling, echter de mortaliteit bleef acceptabel met 2,6\% in de eerste 30 dagen na chirurgische behandeling. We zien echter, dat ondanks de goede lokale controle, de algehele overleving van de patiënten nog steeds te wensen overlaat (overall survival $65 \%$ na 5 jaar), hetgeen vooral te wijten is aan afstandsmetastasen (uitzaaiingen elders in het lichaam). Als we de outcome van deze patiënten willen verbeteren, zal toekomstig onderzoek zich moeten richten op het effectief bestrijden van deze afstandsmetastasen, hetgeen mogelijk bereikt kan worden door effectievere chemotherapeutica, andere timing van chemotherapeutica, of het vroegtijdig (zelfs vóór operatie van de primaire tumor) chirurgisch behandelen van een afstandsmetastase als die al aanwezig is ten tijde van de presentatie van de primaire tumor.

Hoofdstuk 8 van dit proefschrift focust op de identificatie van die patiënten die zo goed gereageerd hebben op neoadjuvante radiochemotherapie, dat het te overwegen is om een meer beperkte chirurgische resectie vit te voeren. Het beperken van de resectie heeft als voordeel dat dit minder belastend is voor de patiënt en dus met minder complicaties gepaard gaat. In een prospectieve multicenter studie werd gekeken hoe goed een expert en een gewone radioloog 
op een MRI na radiochemotherapie konden voorspellen of een tumor zich nu beperkte tot de darmwand en of de klieren geen tumorcellen meer bevatten. De resultaten laten zien, dat als een radioloog zegt dat de tumor zich nu beperkt tot de rectumwand, dit in de meerderheid van de gevallen correct is (90\%). Tevens kan de radioloog met grote zekerheid voorspellen of de lymfklieren negatief zijn geworden (NPV $=0.95$ ). Ook hier is er echter een aanzienlijke overstaging.

De klinische consequentie van de accurate selectie van tumoren die goed hebben gereageerd op radiochemotherapie, is dat het veilig lijkt om bij deze patiënten een meer oppervlakkige resectie te doen. Verder onderzoek naar de daadwerkelijke implementatie hiervan, en de resultaten met betrekking tot outcome zullen in de toekomst worden verwacht.

Met betrekking tot de goede reactie op radiochemotherapie, kan men zelfs nog een stap verder gaan: als MRI in staat is om die patiënten te identificeren, waarbij een complete respons is opgetreden als gevolg van radiochemotherapie (dat wil zeggen: de tumor is geheel verdwenen), dan kan besloten worden om deze patiënten helemaal niet te opereren, maar een traject van strenge follow-up (om een recidief tijdig op te sporen) in te gaan. Een enkele studie die dit al beoogd heeft, toont bemoedigende resultaten. $\mathrm{Er}$ is echter meer prospectief, gerandomiseerd onderzoek nodig om de veiligheid van een dergelijke procedure te onderzoeken.

In de afgelopen decaden is de therapie van het rectumcarcinoom veranderd van één standaard therapie voor iedereen, naar een op maat gemaakte behandeling voor iedere individuele patiënt, op basis van onderzoek van de afgelopen jaren. De resultaten van de studies gepresenteerd in dit proefschrift zullen daar verder aan bijdragen. We hebben echter niet de illusie dat we nu de optimale behandeling van het rectumcarcinoom hebben bereikt, zoals te proeven uit de vooruitblik naar toekomstig onderzoek hierboven beschreven. 


\section{CONCLUSIES}

- Het gebruik van MRI als preoperatieve selectietool voor verschillende risicogroepen binnen de rectumcarcinoompatiënten en de daaropvolgende gedifferentieerde behandeling van deze patiënten leidt tot een toename van het aantal complete resecties in vergelijking met een historische controle, met daarbij voorlopig een bemoedigend laag lokaal recidiefpercentage.

- Preoperatieve MRI met een lymfklierspecifiek contrastmiddel bij rectumcarcinomen kan tumoren die zich beperken tot de rectumwand met een negatieve klierstatus voorspellen met een hoge negatief voorspellende waarde. Dit betekent dat laag-risico tumoren, welke geen preoperatieve radiotherapie nodig hebben, veilig geselecteerd kunnen worden.

* Multidisciplinaire behandeling van het lokaal uitgebreide rectumcarcinoom leidt tot een goede lokale controle van $90 \%$ na 5 jaar, echter met een aanzienlijke morbiditeit veroorzaakt door de behandeling. Afstandsrecidiefvrije en algehele overleving blijven echter teleurstellend en toekomstig onderzoek zal gericht moeten zijn op preventie, vroege detectie en behandeling van afstandsmetastasen.

MRI van het rectumcarcinoom na radiochemotherapie kan veilig die tumoren selecteren die de rectumwand niet meer doorbreken en geen positieve klieren meer hebben. Toekomstig onderzoek kan zich derhalve richten op behandeling met minder vitgebreide chirurgie, zoals een lokale excisie in plaats van een standaard chirurgische resectie. 


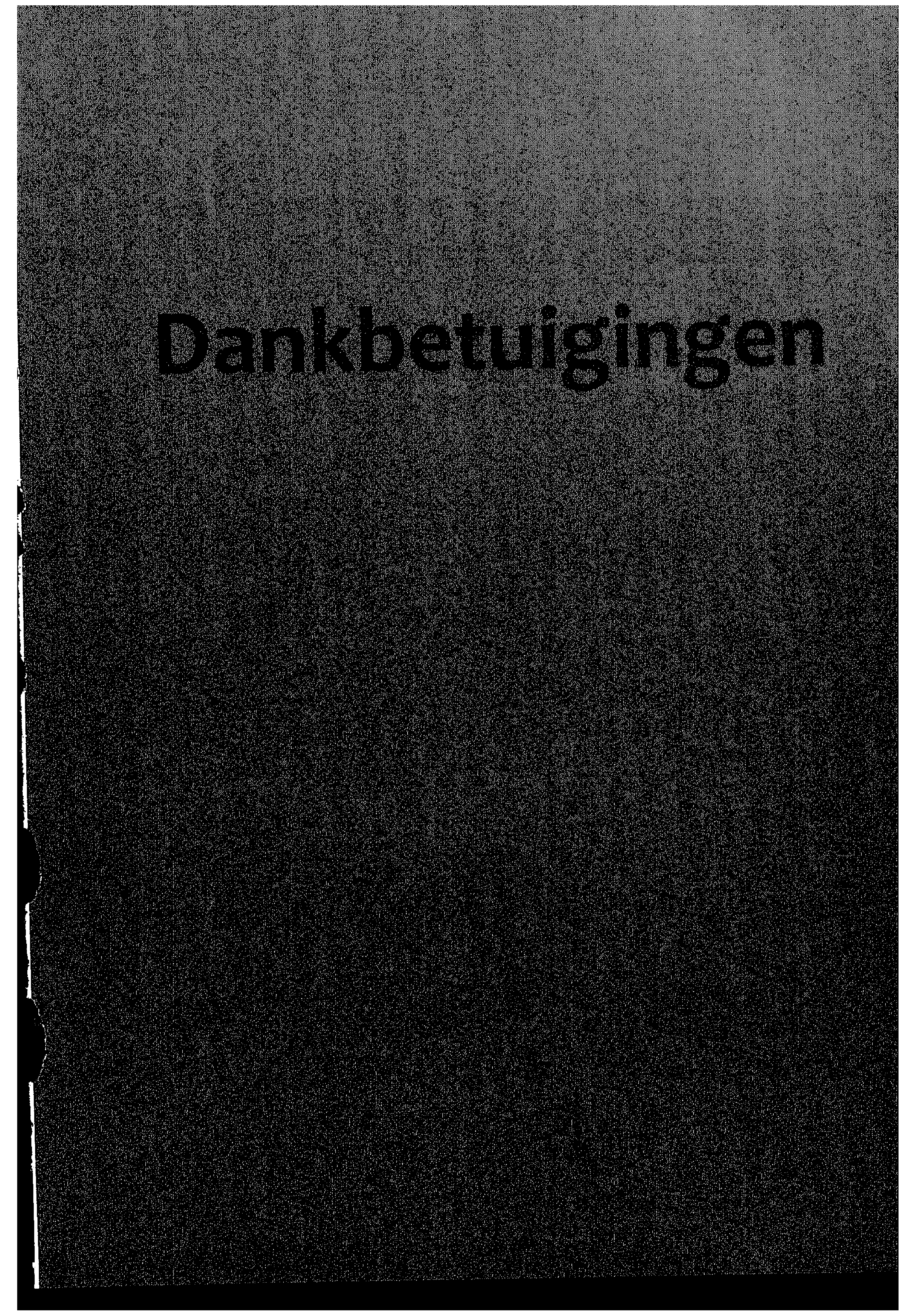




\section{Dankbetuigingen}

\section{DANKBETUIGINGEN}

Hoewel mijn naam eenzaam en alleen op de kaft van dit boekje staat, is de werkelijk wel anders. Een niet te tellen aantal mensen hebben -direct of indirectmeegewerkt aan de totstandkoming van dit proefschrift. Hoewel ik er waarde aan hecht om iedereen persoonlijk te bedanken, kan ik het toch niet laten om enkelen ook in dit 'papieren dankwoord' op te nemen.

Ik wil natuurlijk beginnen met mijn team van promotoren en co-promotor. En hoewel misschien niet volgens de regels, begin ik met de laatste.

Dr. Beets, beste Geerard, hoe druk de kliniek ook was, en hoeveel research er ook nog lag te wachten, tijdens overleg heb ik altijd het idee gehad dat het nooit ongelegen kwam. En hoewel het me op het moment zelf soms irriteerde, liet je me een heleboel dingen zelf bedenken ('daar moet je nog wat aan frutselen/ in grasduinen'), terwijl ik wist dat jij in gedachte al 5 stappen verder was... Achteraf denk ik dat je je daarin een goede wetenschappelijke begeleider toonde. Je bent bovendien een belangrijk voorbeeld voor me, als chirurg die met passie zijn vak vitoefent en ook nog eens verschrikkelijk goed is in dat vak. Ik hoop dan ook dat ik de komende tijd op klinisch vlak de nodige feedback van je mag ontvangen, omdat dat voor mij heel waardevol is.

Prof. dr. Beets-Tan. Regina, vanaf dag één heb je mij met je enorme enthousiasme en niet te stoppen energie gemotiveerd voor dit onderzoek en de wetenschap in het algemeen. Ik heb grote bewondering voor je bevlogenheid en doorzettingsvermogen, ook als het tegen de stroom in is, én voor dat hele belangrijke menselijke dat je zo nu en dan weer benadrukt: 'We doen het voor al die mensen, die móeten we beter maken....'. Je bent niet alleen mijn wetenschappelijke begeleider geweest, maar eigenlijk ook een beetje mijn personal coach. Bedankt voor jouw wijze lessen. Ik hoop dat mijn proefschrift voor mij het begin is van een wetenschappelijke carrière, waarin ik nog veel met je mag samenwerken.

Prof. dr. van de Velde, het is voor mij een eer dat $\mathrm{u}$ bereid bent geweest deel uit te maken van mijn promotieteam. Bedankt voor de betrokkenheid bij onze rectUM studie en de kritische blik op mijn werk in de afgelopen jaren. 
Prof. dr. von Meyenfeldt, uw enthousiasme voor het chirurgisch vak tijdens uw optreden als tutor van mijn onderwijsgroep ergens halverwege mijn studie heeft toch zeker meegewogen in mijn keuze voor de opleiding. En dan mocht ik óók nog uw begeleiding genieten gedurende dit promotie-traject... $U$ zult wel begrijpen: mijn dank is groot!

Prof. dr. Dejong, bedankt voor het aanvaarden van de rol van voorzitter van de beoordelingscommissie van mijn proefschrift, en uw goede adviezen. Prof. dr. Stroobants, Prof. dr. Wildberger, Prof. dr. Kruitwagen en Dr. de Wilt, hartelijk dank voor de kritische en dus zeer waardevolle beoordeling van mijn proefschrift.

In de jaren van mijn promotieonderzoek heb ik nauw samengewerkt met mijn 'radiologische tegenhanger', $\operatorname{Dr}(\mathrm{s})$. M.J. Lahaye, die om die reden natuurlijk ook naast mij staat tijdens mijn verdediging. Max, we mogen het wel lief en leed noemen, dat we gedeeld hebben. Jouw positieve manier van tegen zaken aankijken maakte het een paar leuke jaren. Ik bewonder de ultrarelaxedte manier waarop jij het altijd klaarspeelt om kwaliteit af te leveren. Daarnaast dank ik je, dat je mijn externe relativerend vermogen was. $\mathrm{lk}$ weet zeker dat ik je ga missen als naaste collega, ook al heb ik nu al een paar maanden geoefend. Gelukkig krijg ik er koud staal voor terug... wat wil een mens nog meer?

Het opzetten en uitvoeren van een multicenter studie vergt heel veel van vele partijen.

Allereerst ben ik dank verschuldigd aan GROW School for Oncology \& Developmental Biology, dat de basis was van waaruit we konden handelen.

Verder hebben wij in deze multicenter studie nauw samengewerkt met met name de afdelingen Chirurgie en Radiologie, maar daarnaast ook met Pathologie van het Viecuri Medisch Centrum voor Noord-Limburg, het Laurentius ziekenhuis in Roermond en het St. Jans Gasthuis in Weert. Dank aan Dr. Konsten, van Berlo, Nijhuis, Estourgie, Rongen, Leijtens, Jansen en Verkooijen voor de inclusie van patiënten, en de enthousiaste betrokkenheid bij de studie. Ik ben te laat om mijn dank uit te spreken aan Dr. Debets. Toch wil ik zijn enthousiasme voor deelname aan het onderzoek, maar met name ook de inzet om voor ieder van zijn patiënten 


\section{Dankbetuigingen}

met volle aandacht de beste behandeling na te streven niet onvermeld laten. Voor mij was dat een belangrijk voorbeeld.

Dr. Post, Bilderbeek, Opdenakker en Dohmen, veel dank voor het geduld iedere minuscule klier te scoren. Dr. Willig en Dr. Kwee, een aantal keren heb ik u op de vingers mogen komen kijken tijdens het vitsnijden van een preparaat, of hebt $u$ met mij coupes bekeken, ik heb daar veel van geleerd. Dank daarvoor!

Ook in het MUMC hebben wij gemerkt dat er veel animo was voor onze studie. Dankzij een zeer gemotiveerde GE-Onco staf en chivo's, -in volstrekt willekeurige volgorde- Dr. Bemelmans, van Dam, Keymeulen, Bouvy, van Gemert, Olde Damink, Huizinga, Oosterkamp, Stoot (jr.), Prof. Baeten, Greve en Dejong, alsook een behoorlijk aantal chirurgisch assistenten was de inclusie bij de afdeling Chirurgie moeiteloos! Hetzelfde geldt voor alle artsen van de MDL, die telkens weer belden als ze een patiënt 'gevangen' hadden op de scopiekamer. Ook bij de afdeling Radiologie, in het begin nog onder de bezielende leiding van Prof. van Engelshoven, was er een behoorlijke club die iedere keer weer klaarstond als wij weer een MRI wilden bekijken. In het begin was dat met name Roy Vliegen, later kwam Alette Daniels-Gooszen ons tijdelijk versterken -én mij een paar wijze lessen over beroepskeuze leren...- en nu zijn het Frans Bakers (hoezo 'nonexpert') en Vincent Cappendijk die hun wijze blik over de scans laten gaan. Allen bedankt! En niet te vergeten alle assistenten van de Pathologie, voor het iedere keer weer uitsnijden van de preparaten (en het wedstrijdje om meer klieren te vinden als de MRI, klasse!) alsook Dr. Driessen en Prof. dr. de Brüne; altijd beschikbaar voor tekst en uitleg. Natuurlijk wil ik de andere belangrijke schakels in het multidisciplinair team niet overslaan: Dr. Jansen, Lammering en De HaasKock, dank voor de kritische blik op ons werk!

Van onschatbare waarde was bovendien al het werk verricht door verpleegkundigen, MRI-laboranten en administratief personeel in de verschillende ziekenhuizen. In Venlo hebben de MRI-laboranten tussen alle andere werkzaamheden door ook nog de Sinerem-infusen toegediend, én ervoor gezorgd dat wij daar altijd meteen van op de hoogte waren. In Roermond werd dit werk verzet door de verpleegkundigen op afdeling $A_{1}$ (tegenwoordig $C_{1}$ ), en in Weert op het Dagcentrum. Overal konden we rekenen op medewerking en 
werden we direct op de hoogte gesteld bij vragen/problemen. Dit geldt ook voor de laboranten die de scans maakten (voor Marja Stassen was het zelfs niet teveel werk om zelf aan de sequenties te sleutelen...) en het administratief personeel dat er altijd voor zorgde dat we binnen de kortst mogelijke tijd de cdtjes met de scans binnenkregen. Het was een prettige samenwerking. Voor wat betreft de mensen in het MUMC, geldt naast de al genoemde laboranten nog een special thanx voor Guus Groen, die altijd een spoedplek op de MRI had voor onze patiënten, en alle andere dames van de balie, de dames van de typekamer, voor de snelle service, Arno Magermans en Frans Kusters, voor al die cdtjes op het PACS zetten, Herman Roes, en natuurlijk Etienne Lemaire en Henk Schoenmakers, voor de hulp met de MR-protocollen in de andere ziekenhuizen...

Buiten alle handen die geholpen hebben bij het opzetten van deze studie moet ik niet vergeten te vermelden hoe belangrijk alle patiënten waren. In een moeilijke tijd, net na het stellen van de diagnose rectumkanker, namen zij toch de moeite om verder te kijken dan alleen hun eigen belang, en keken zij naar wat ze konden betekenen voor alle rectumkankerpatiënten die na hen kwamen, door deel te nemen aan wetenschappelijk onderzoek. Het contact met hen was bovendien niet alleen belangrijk voor mij als onderzoeker en arts, maar ook als mens: ik heb geleerd van alle dapperheid en optimisme in moeilijke tijden. Allen hartelijk dank voor deelname!

Fons Kessels, natuurlijk ben ik je in de eerste plaats dankbaar voor telkens weer die geduldige vitleg over de meest basale lessen in statistiek, die ik dan toch weer niet kon onthouden. Maar daarnaast heb je me veel geleerd over hoe de zaken eraan toe gaan in de arena die het ziekenhuis (en: de onderzoekswereld) heet. Jij had daar altijd goed overdachte ideeën over en wilde die graag met me delen. Ik heb veel van je geleerd.

Evelien, Joyce, Johanna, Yvonne, Brigitte, Christianne, Monique, Elfie, Astrid en Eveline dank voor alle hulp bij het veroveren van plekjes in de agenda's van begeleiders, hulp bij correspondentie en zo verder. 


\section{Dankbetuigingen}

En dan de mensen in hetzelfde schuitje: mijn collega's onderzoekers:

Elleke, weliswaar in Eindhoven, maar toch echt part of our team. Dat blijkt ook wel op deze dag, waarop we samen gaan verdedigen en feesten. Het was leuk om met je samengewerkt te hebben. Het was nog leuker om samen op congres te gaan en het is misschien wel het leukst dat jij nu ook aan de slag bent in het MUMC...kan ik je tenminste nog regelmatig lastig vallen op het dienstsein...

Doenja, jij volgt ons (of eigenlijk Max) op. Ik heb al een tijdje kunnen genieten van jouw managementkwaliteiten en onderzoeksvaardigheden, dus ik heb er het volste vertrouwen in dat het helemaal goed komt!

Monique, heel veel succes met jouw onderzoeksproject! Je hebt er een lange adem voor nodig, maar het is prachtig onderzoek, en heel actueel. Ik kijk uit naar de resultaten!

Al mijn kamergenoten en één-of-meerdere-kamertje(s)-verder-genoten van de afgelopen jaren, Jaap (kan je geen ongelijk geven dat je naar een paar kamers verder gevlucht bent...maar om nou helemaal naar NY te vertrekken....), Rianne (die stelling van het compliment is echt fantastisch), Robert, Suzanne (gelukkig nu nog een beetje collega's in de kliniek), Robbert-Jan, Marlies, Karolien, Walter,

164 Eline, Gerrit, Quido, Marianne, Robbert, Nils, Vera, Dave, Marc L., Maarten, Luc, Rob, Chris, Koen, Roel, Marielle, Cécile: dank voor de collegialiteit en gezelligheid. Marc G. en Jos: dank voor jullie altijd beschikbare EHBC (Eerstehulp-bij-computerproblemen).

Mascha, hoewel het er nog niet van gekomen is (maar we hebben nog een paar jaartjes): leuk dat we nu samen cursussen kunnen doen.

En natuurlijk blijven er dan ook een paar mensen over van buiten de muren van het ziekenhuis.

Tan Amigos, bij jullie is het voor mij altijd even verstand op nul en genieten. Van de andere kant was er ook altijd de gelegenheid om te praten over de moeilijkheden van onderzoek doen en de onzekerheid over een opleidingsplek. Jullie zorgden dan voor de broodnodige relativering. Dank jullie wel allemaal.

Hilke, ik sla je waarschuwingen in de wind, en begin aan de opleiding. Koppig dus, net als jij. Toch waren we altijd een goede combinatie tijdens onze gezamenlijke trips, maar waarschijnlijk alleen omdat onze neuzen altijd dezelfde kant op stonden. Dingen zijn veranderd, en in drukke tijden kan zelfs een woonplaats een 
uur verderop een belemmering zijn om elkaar met enige regelmaat te zien. Gelukkig sta je op deze voor mij belangrijke dag aan mijn zijde! Dank voor jouw vriendschap.

Dirk, het moment is aangebroken dat ik echt weer meer tijd heb... Het wordt tijd dat we weer terug gaan naar de tijd dat een fles openmaken ook een fles leegdrinken betekende.... hoewel daarna slingerend op de fiets naar huis (GoudaMaastricht;)) tegenwoordig toch wat lastig wordt.

Papa, als mijn voorbeeld daag jij me uit om steeds een treetje hoger te komen. Hopelijk gaat -met betrekking tot de tijd na mijn promotie- de uitspraak 'goed voorbeeld doet volgen' ook in dit geval op...

Lieve mama, waarschijnlijk weet je niet eens wat voor een rots in de branding je bent voor mij, en misschien vergeet ik het vaak ook wel te zeggen, tussen al het tegensputteren door.

Thijs, Leon en Cas, dank voor de relativering op z'n tijd ('Sanne, waarom doen we dit?').

Tot slot, liefste Xavier, 'Gravitation cannot be held responsible for people falling in love' (Albert Einstein). Ik denk ook niet dat we het moeten proberen te verklaren. Feit is dat je er altijd voor me was vanaf die eerste dag in Gent, er altijd bent voor al mijn vreugde en verdriet en er altijd zult zijn voor de uitdagingen die we samen nog tegemoet gaan. En dat maakt alles de moeite waard. 


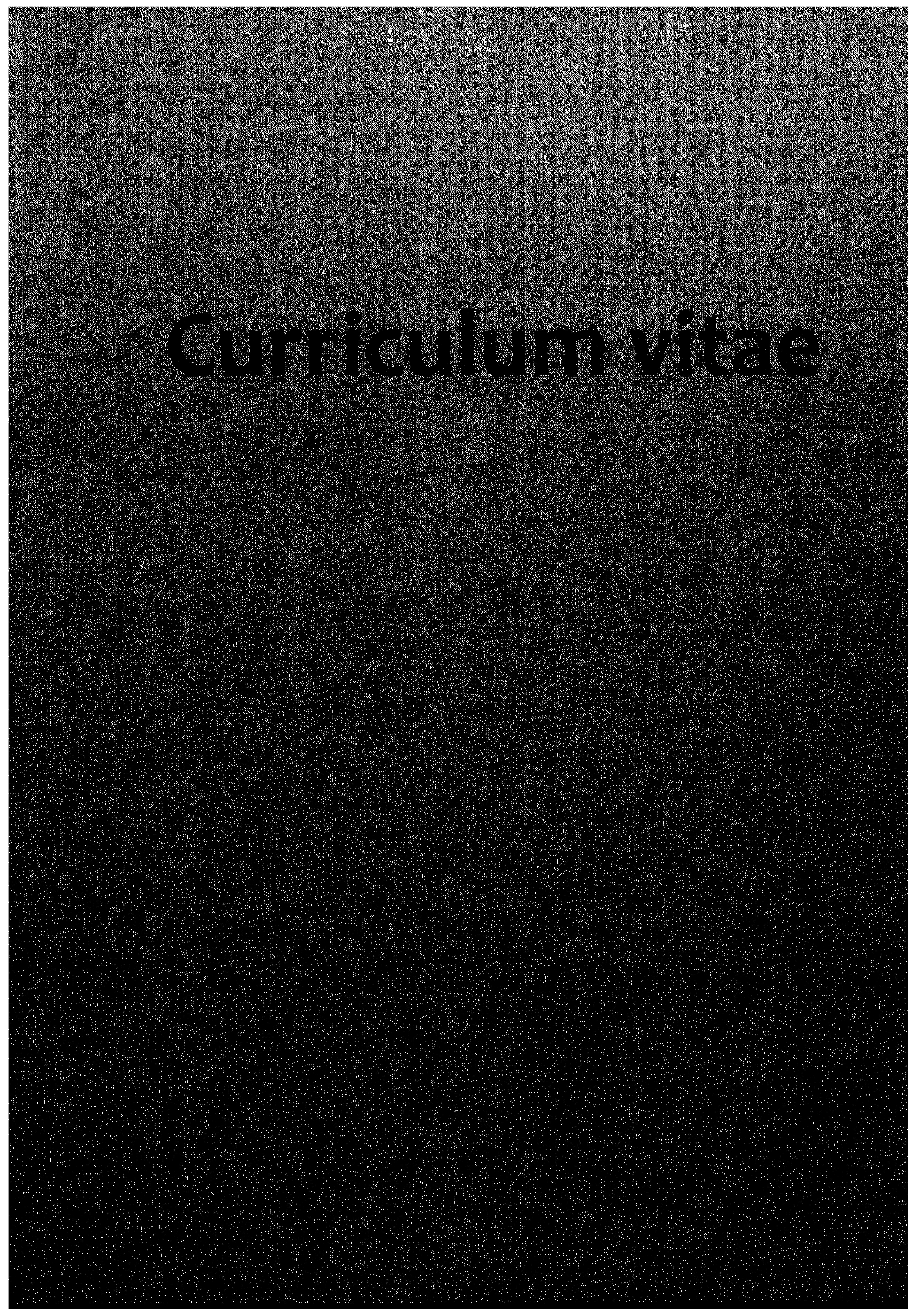




\section{Curriculum Vitae}

\section{CURRICULUM VITAE}

Sanne M.E. Engelen was born on July 14, 1980 in Boxmeer, The Netherlands. She graduated from the Merlet College Land van Cuijk in Cuijk in 1998 (Atheneum). In the same year, she started her medical training at the University of Maastricht, Faculty of Medicine. During her internships, she visited the Perinatal Research Center at the University of Alberta, Edmonton, for her research elective 'Control of birth'. Furthermore, she was secretary of Ko-beraad (the association for medical interns), and editor of 'Kozijn', the associations' 3-monthly periodical. She graduated from Medical School in September 2004 (Cum Laude). Immediately thereafter, she became a MD, PhD-student at GROW School of Oncology and Developmental Biology, University of Maastricht and the departments of Radiology and Surgery, Maastricht University Medical Center. During her four-year research project on tailored treatment of rectal cancer, she was nominated for the 'Radiologendagenprijs 2006', a prize for the best abstract on the annual meeting of the Radiological Society of the Netherlands (Kaatsheuvel). Furthermore, she won the Incentive Prize of the Pélerin Symposium 2007, Maastricht. Her research project resulted in this thesis. In July 2008 she started her surgical residency at the University Hospital Maastricht (Prof. dr. C.H.C. Dejong). For the last part of her residency (starting July 2011), she will work at the Catharina Hospital in Eindhoven (Dr. G.A.P. Nieuwenhuijzen). 